\title{
A BIOINSPIRED MICRO-COMPOSITE STRUCTURE
}

\author{
by \\ LI CHEN \\ Submitted in partial fulfillment of the requirements \\ For the degree of Doctor of Philosophy \\ Dissertation Advisor: Prof. Roberto Ballarini \\ Department of Civil Engineering \\ CASE WESTERN RESERVE UNIVERSITY
}

August 12, 2005 


\section{CASE WESTERN RESERVE UNIVERSITY \\ SCHOOL OF GRADUATE STUDIES}

We hereby approve the dissertation of

\section{Li Chen}

candidate for the Ph.D. degree *.

(signed) Roberto Ballarini

(chair of the committee)

Arthur Heuer

Steven Izen

Harold Kahn

Christian Zorman

(date) $\underline{05 / 24 / 2005}$

*We also certify that written approval has been obtained for any proprietary material contained therein. 


\section{Table of Contents}

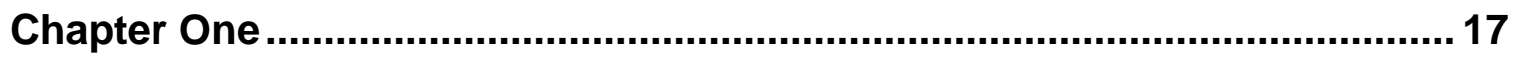

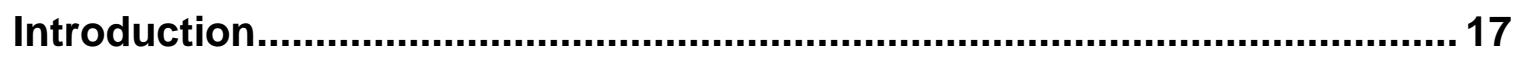

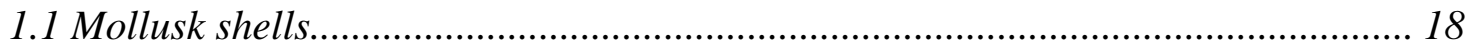

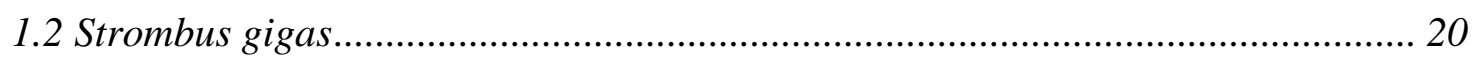

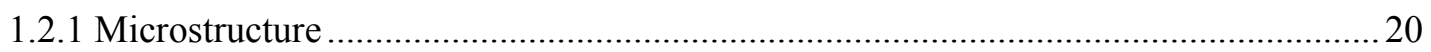

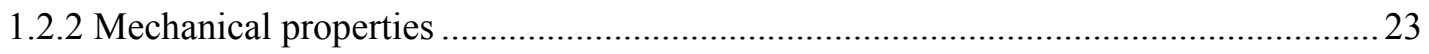

1.3 Biomimetics_synthetic multi-layer materials ...................................................... 26

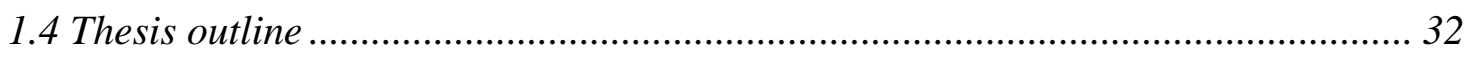

Chapter Two

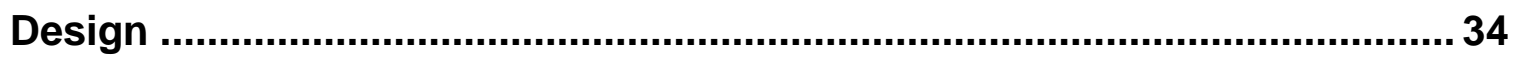

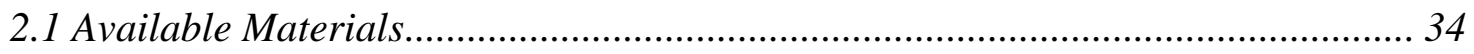

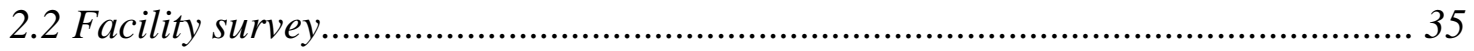

2.3 Design of the synthetic microstructure ……………................................................ 41

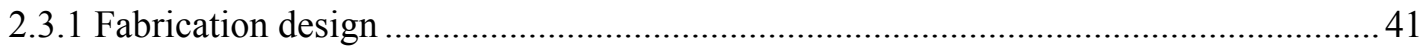

2.3.2 Microstructure and photomask design ..................................................................... 50

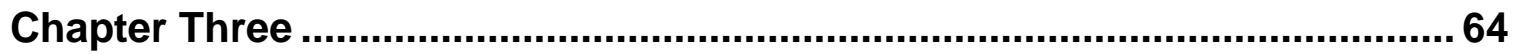

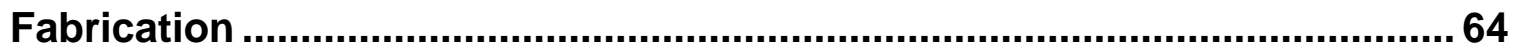

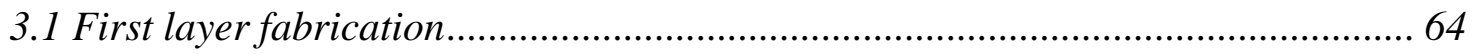

3.2 Sputtered silicon film …………………………........................................... 71

3.2.1 Sputtering tool and silicon target ………………………………………………..... 71

3.2.2 Deposition rate and residual stresses.................................................................... 71

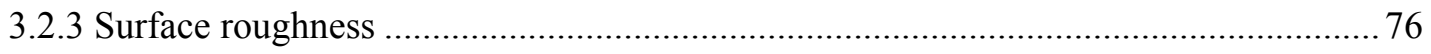

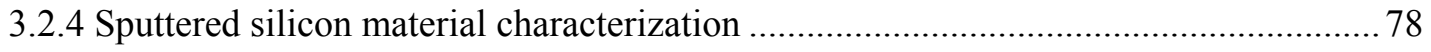


3.2.5 Etching sputtered silicon films

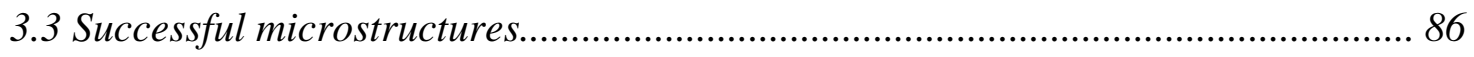

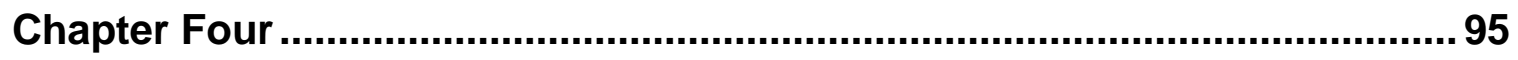

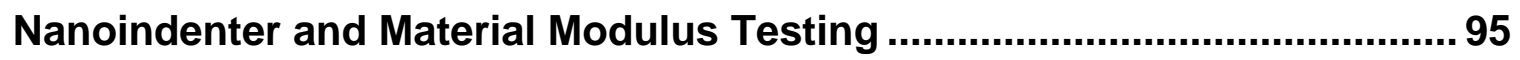

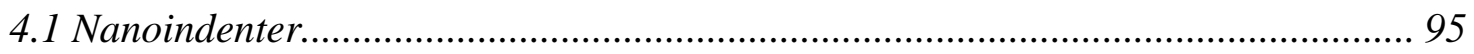

4.2 Measurement of the Elastic Modulus............................................................... 101

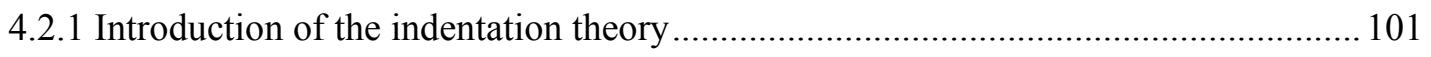

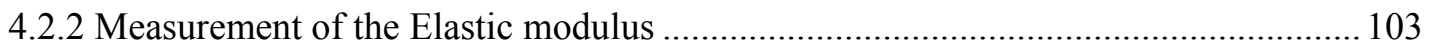

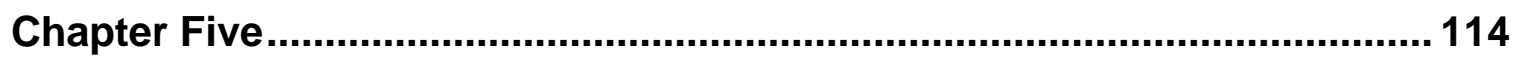

Mechanical Properties of the Micro-composite Structure ....................... 114

5.1 Instrument control for data acquirement.................................................... 114

5.2 Mechanical properties of the micro-composite structure ................................... 121

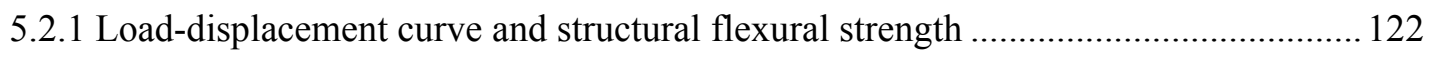

5.2.2 Tunnel cracking and crack bridging mechanisms in the microstructure .................... 134

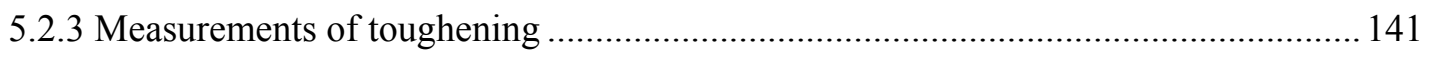

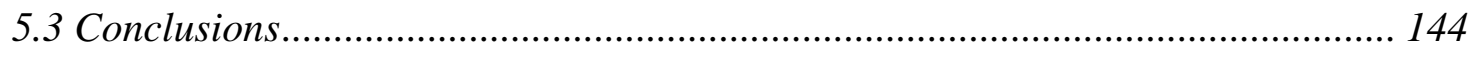

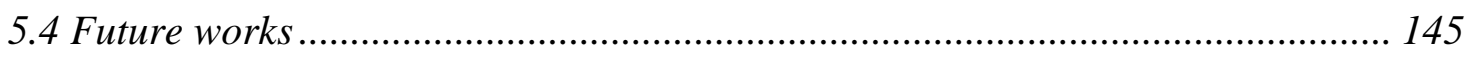

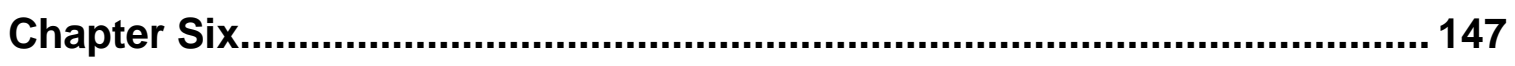

A Model for Strengthening of Polysilicon Devices .................................. 147

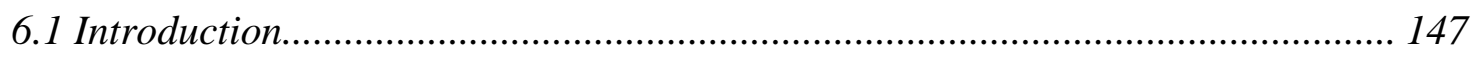

6.2 Plastic Flow in Amorphous Silicon and the Drucker-Prager Model ................... 148

6.3 Simulations of Cyclic Loading on Notched Polysilicon Specimens..................... 151

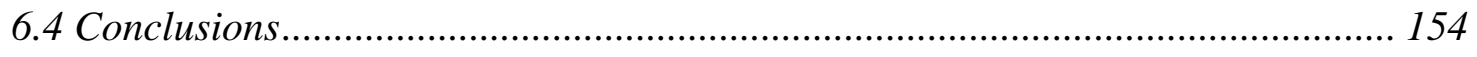

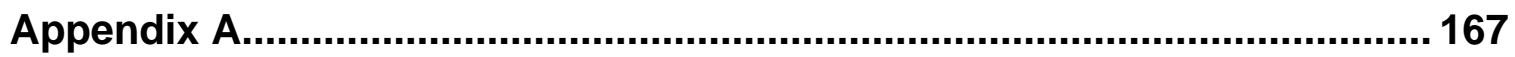




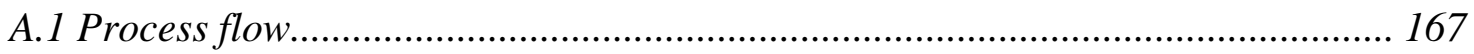

A.2 Silicon etching recipe with $\mathrm{SF}_{6} / \mathrm{O}_{2}$ based plasma ......................................... 170

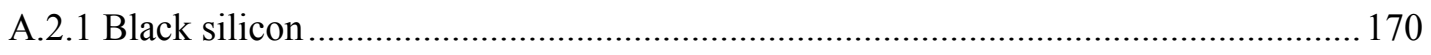

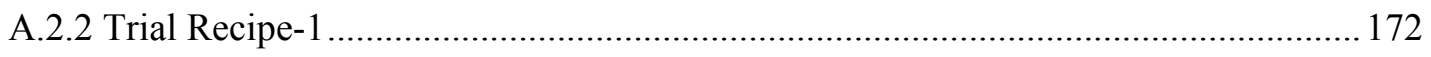

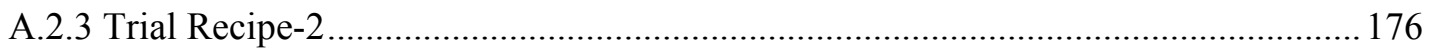

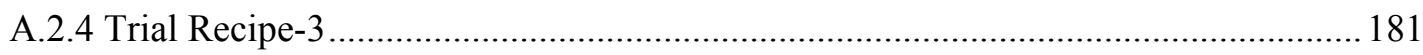

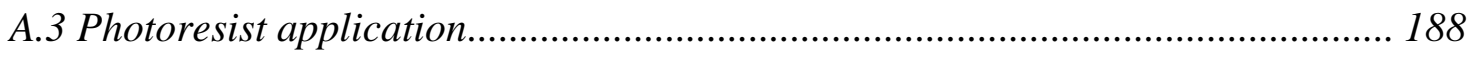

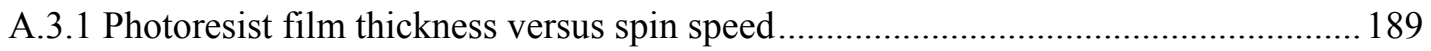

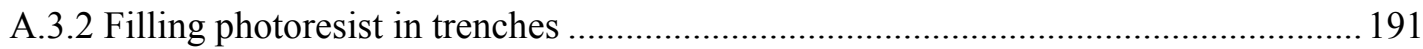

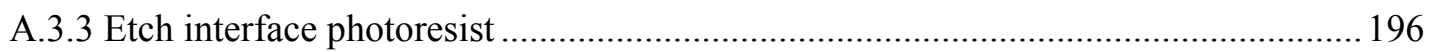

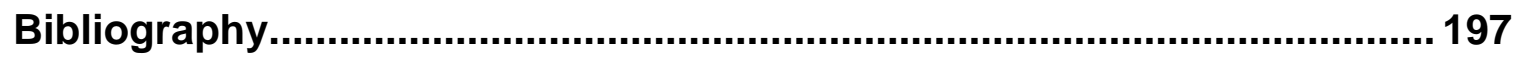

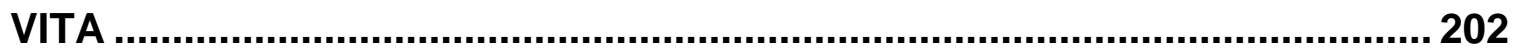




\section{List of Tables}

Table 3.1 Etching results for the polysilicon film with the chlorine-based recipe and the

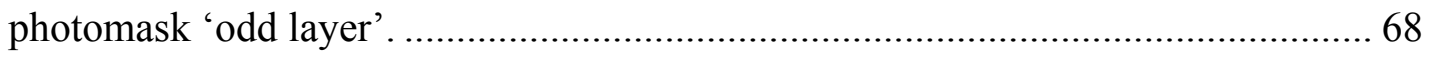

Table 3.2 Impurity concentrations in silicon target. ............................................... 71

Table 3.3 Deposition rates of the sputtered silicon film using Discover24.................... 73

Table 3.4 Deposition rates and residual stresses for the sputtered silicon films using different process parameters with Discover24............................................. 75

Table 3.5 Surface roughness of sputtered silicon films. ........................................... 77

Table 3.6 Etching results of the sputtered silicon film with chlorine-based recipe and

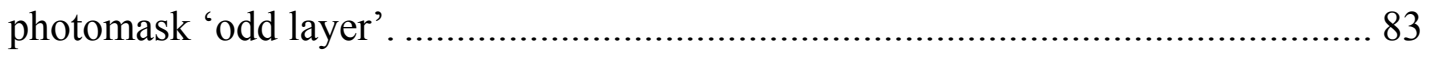

Table 3.7 Etching results of the sputtered silicon film with chlorine-based recipe and

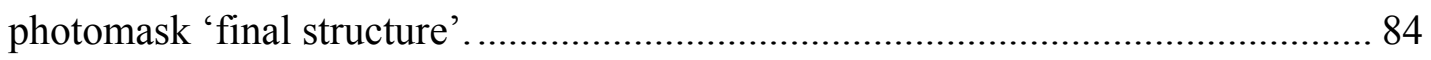

Table 3.8 Spin coater parameters for depositing 4 $\mu \mathrm{m}$ thick AZ9260 photoresist. ........... 89

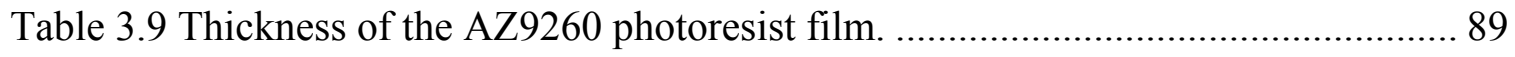

Table 3.10 Final processes for etching three-layer silicon films with trial recipe-3........ 90

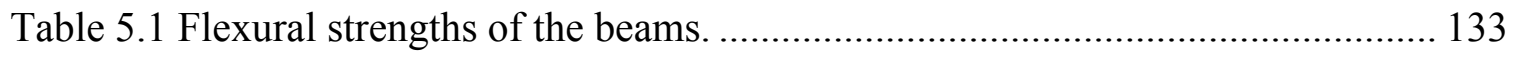

Table 5.2 Dissipated energy of the microstructures............................................... 142

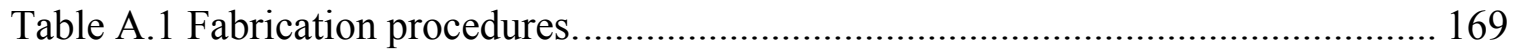

Table A.2 Details of chlorine-based silicon etching recipe. ..................................... 173

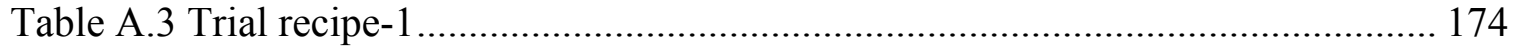

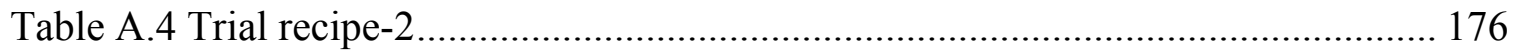

Table A.5 Etching results of the polysilicon film (trial recipe-2 and photomask 'odd

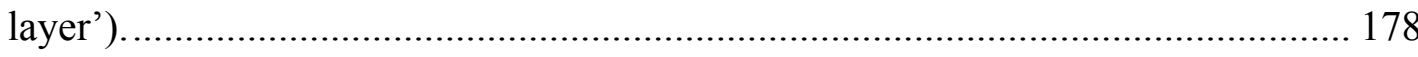


Table A.6 Etch rates of the polysilicon film using trial recipe-2. 180

Table A.7 Etching results of the sputtered silicon film (trial recipe-2, photomask 'odd layer'). 181

Table A.8 Etch rates of the sputtered silicon film (trial recipe-2, photomask 'odd layer').

Table A.9 Trial recipe-3 182

Table A.10 Etching results of the polysilicon film (trial recipe-3 and photomask 'odd layer'). 184

Table A.11 Etch rates of the polysilicon film (trial recipe-3 and photomask 'odd layer'). 184

Table A.12 Etching results of the sputtered silicon film (trial recipe-3 and photomask 'odd layer'). 184

Table A.13 Etch rates of the sputtered silicon film (trial recipe-3 and photomask 'odd layer'). 184

Table A.14 Etching results of the sputtered silicon film (trial recipe-3 and photomask 'final structure'). 186

Table A.15 Etch rates of the sputtered silicon film (trial recipe-3 and photomask 'final structure') 186

Table A.16 Etching results of the oxide film using trial recipe-3 ............................. 187

Table A.17 Etch rate of the oxide film using trial recipe-3 ..................................... 187

Table A.18 Summary of the etch rates for different materials using the trial recipe-3. . 188

Table A.19 Photoresist thicknesses vs. spinning speed. 189 
Table A.20 Spin coater parameters for depositing $1.2 \mu \mathrm{m}$ thick photoresist film, Shipley

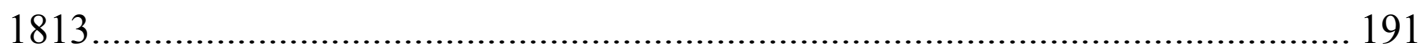

Table A.21 Spin coater parameters for depositing $4.1 \mu \mathrm{m}$ thick Shipley 1813 photoresist. 192

Table A.22 Spin coater parameters for depositing $0.9 \mu \mathrm{m}$ thick Shipley 1813 photoresist. 193

Table A.23 Spin coater parameters for depositing $1.05 \mu \mathrm{m}$ thick Shipley 1813 photoresist. 195

Table A.24 Etching results and etch rates for polysilicon and photoresist using $\mathrm{CHF} 3 / \mathrm{O}_{2} / \mathrm{He}$ recipe 


\section{List of Figures}

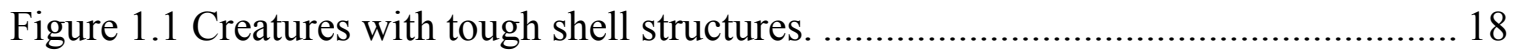

Figure 1.2 Schematic representations of the Crossed-lamellar aragonite....................... 19

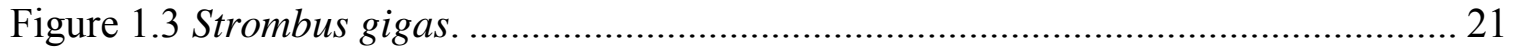

Figure 1.4 (a) - A schematic representation of the crossed lamellar microstructure of the Strombus gigas. (b), (c) and (d) - SEM images of the fracture surface of a bend specimen taken at increasing magnification. (O, M and I refer to outer, middle and inner layers) ${ }^{[6]}$ 22

Figure 1.5 (a) SEM image of the interfaces separating second-order lamellae (CC) and the interfaces separating first-order lamellae (BCCB and BCB). (b) TEM image of a first-order interface. (c) TEM image of a second-order interface. (d) TEM image of a

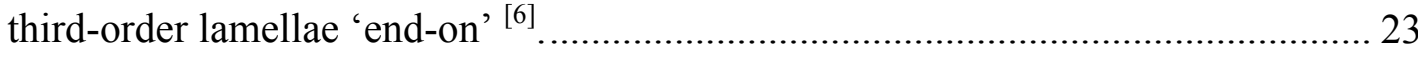

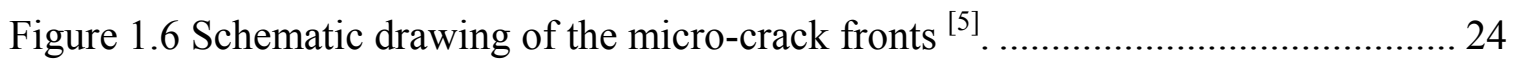

Figure 1.7 Load-Displacement curves for tough middle layer and weak inner layer

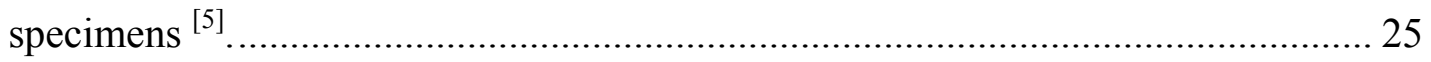

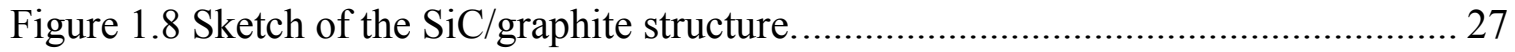

Figure 1.9 Load-deflection curve of a notched SiC/graphite structure ${ }^{[11]}$..................... 28

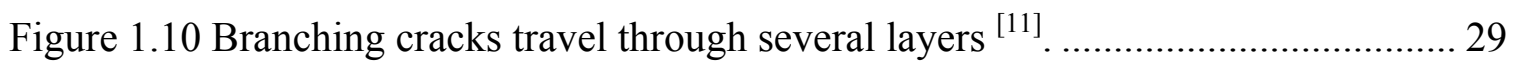

Figure 1.11 Load-deflection curves for six-layer epoxy without interlayer, with one

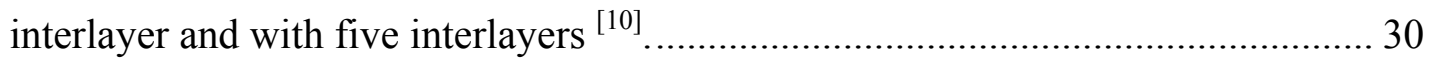

Figure 1.12 Cross-section of alumina/aluminum composite parts made by free-forming porous ceramic and backfilling with metal. (a) Section through pore region. (b)

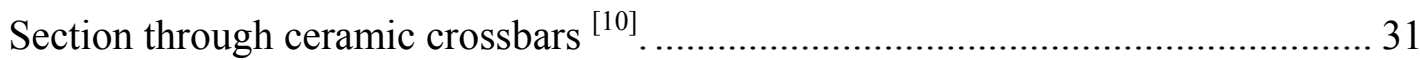


Figure 1.13 Load-deflection curves for alumina-aluminum composites under a four-point bend loading with the ceramic surface in tension ${ }^{[10]}$. 32

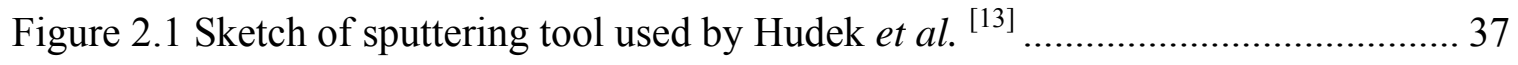

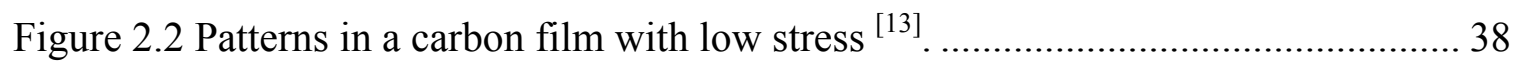

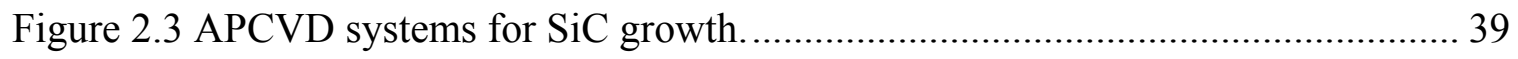

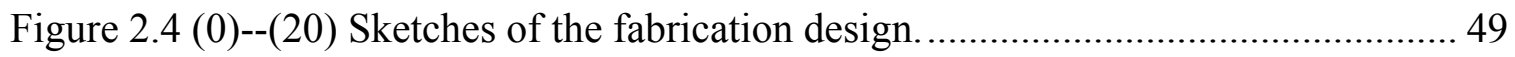

Figure 2.5 Illumination of the microstructure and the load direction.......................... 52

Figure 2.6 Laminate with silicon matrix and photoresist bars in 1-2 plane.................... 53

Figure 2.7 Principal material directions rotate an angle $\theta$ with respect $\mathrm{X}$-axis in $\mathrm{X}-\mathrm{Y}$

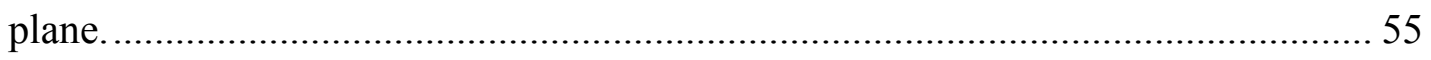

Figure 2.8 Sketch of the simplified microstructure. .............................................. 58

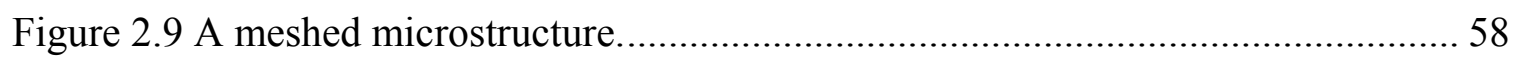

Figure 2.10 (a) Beam deformation (b) Load-deflection curve for the loading point........ 59

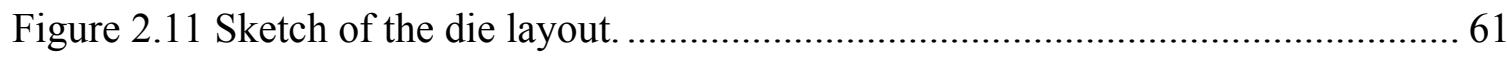

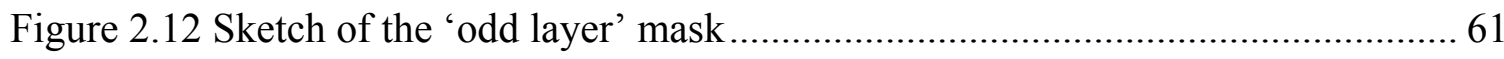

Figure 2.13 Sketch of the two overlapped masks, mask 'odd layer' and mask 'even layer'.

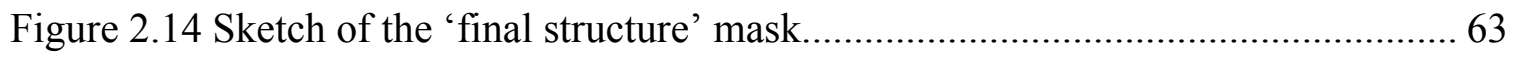

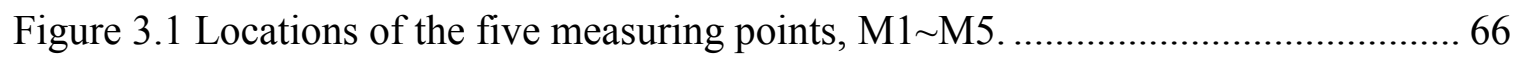

Figure 3.2 Top view of the photoresist etching mask with trenches. ........................... 67

Figure 3.3 Black silicon in the etched trench area (polysilicon films, chlorine-based

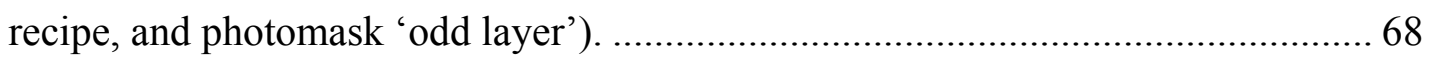


Figure 3.4 Etched areas around the anchor (chlorine-based recipe and photomask 'final structure') 70

Figure 3.5 Curvature map and calculated residual stress for the sputtered silicon film deposited with 200Watts of power and 5mTorr of pressure. 75

Figure 3.6 Surface roughness of the sputtered silicon film with thickness of $15338 \AA$ (tapping mode AFM). 77

Figure 3.7 Element profiles in the sputtered silicon film........................................ 79

Figure 3.8 X-ray diffraction pattern of the sputtered silicon film................................ 80

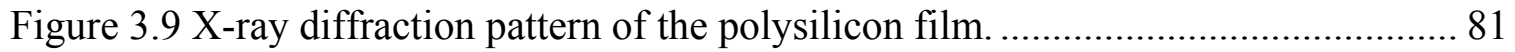

Figure 3.10 Black silicon in the etched area (sputtered silicon films, chlorine-based recipe, and photomask 'odd layer'). 83

Figure 3.11 Black silicon around the anchor area (sputtered silicon film, chlorine-based recipe, and photomask 'final structure'). 84

Figure 3.12 Top view of the trench area after etching the first structural layer. 87

Figure 3.13 Top view of the trench area after etching the second structural layer.......... 88

Figure 3.14 Top view of the trench area after etching the third structural layer. 88

Figure 3.15 Top view of the three-layer silicon beam after being etched for 4min.using trial recipe-3 92

Figure 3.16 Top view of the three-layer microstructure after being released................. 93

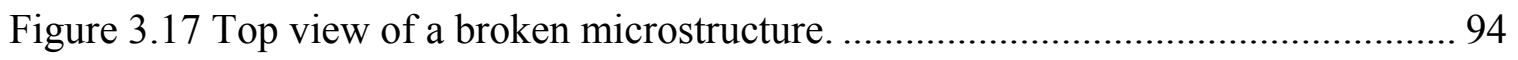

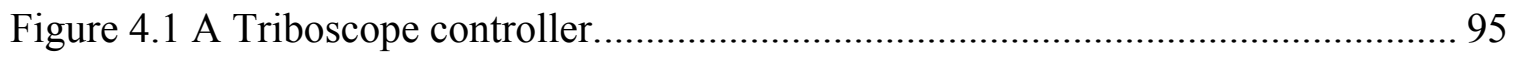

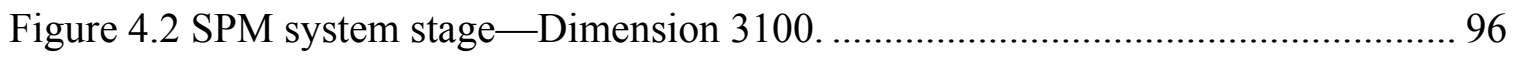

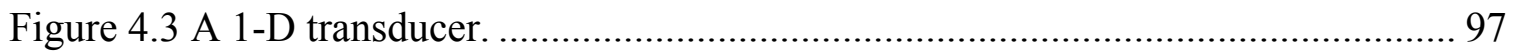




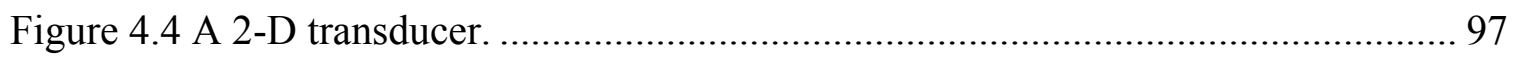

Figure 4.5 Indentation tip mounted on a 2-D transducer............................................... 98

Figure 4.6 (a) Triboscanner; (b) Triboscanner with 2-D transducer................................ 99

Figure 4.7 SPM stage with Nanoindentation transducer and tip. ................................. 100

Figure 4.8 Load-displacement curve of the indentation. .............................................. 101

Figure 4.9 Schematic representation of a cross-section of an indentation...................... 102

Figure 4.10 Berkovich indentation tip................................................................ 104

Figure 4.11 Area function of the Berkovich indentation tip........................................ 105

Figure 4.12 A typical load function for the indentation test.......................................... 106

Figure 4.13 Testing results for standard fused quartz. (a) $3000 \mu \mathrm{N}$; (b) $6000 \mu \mathrm{N}$; (c)

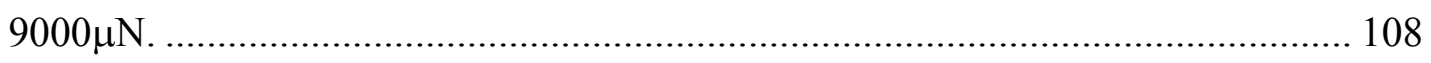

Figure 4.14 Testing results for the sputtered silicon film. (a) $3000 \mu \mathrm{N}$; (b) $6000 \mu \mathrm{N}$; (c)

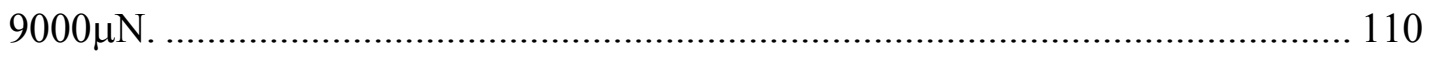

Figure 4.15 SPM image of the sputtered silicon surface after the indentation test. ....... 111

Figure 4.16 Load-displacement curve of an indentation test for the photoresist film.... 113

Figure 4.17 SPM image of the photoresist film surface after indentation test. .............. 113

Figure 5.1 Side view of the flat punch tip................................................................. 114

Figure 5.2 Magnified cross-section of the tip's free end. ............................................ 115

Figure 5.3 Image of the tip, its shadow and the nearby substrate captured by the vision

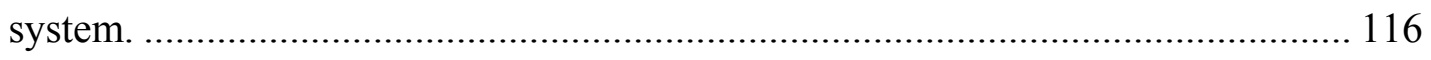

Figure 5.4 Displacement control function. ………………..................................... 118

Figure 5.5 Calibration of the lateral force............................................................ 119

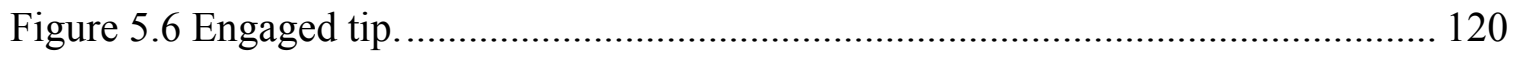


Figure 5.7 Released beams. (a) $200 \mu \mathrm{m}$ long beam; (b) $300 \mu \mathrm{m}$ long beam.

Figure 5.8 Curves of the normal force and normal displacement versus the lateral displacement. 123

Figure 5.9 Data points of the friction versus the lateral displacement. 125

Figure 5.10 Load-displacement curve of the free end of the flat punch tip................... 126

Figure 5.11 Lateral load-displacement curves for the $20 \mu \mathrm{mx} 200 \mu \mathrm{m}$ beams. 132

Figure 5.12 SEM image of the beam--d24r15c23 after the bending test and the corresponding load-displacement curve. 135

Figure 5.13 SEM images of the beam-d24r13c11 after the bending test. (a) Middle layer side; (b) Inner layer side. 136

Figure 5.14 SEM images of the beam-d24r07c23 after the bending test. (a) Middle layer side; (b) Inner layer side. 137

Figure 5.15 Beam-d24r23c11 with part of the top layer broken away and the corresponding load-displacement curve. 139

Figure 5.16 (a) Micro-probe position before pushing the beam. (b) Micro-probe position after a catastrophic failure 140

Figure 5.17 Schematic illustration of the extended load-displacement curve in the displacement portion of $10 \mu \mathrm{m} \sim 16 \mu \mathrm{m}$ 141

Figure 6.1 Schematic illustration of cyclic loading applied to notched polysilicon MEMS specimens 155

Figure 6.2 Effects of mean stress and stress amplitude on nominal strength of notched polysilicon MEMS structures. 156 
Figure 6.3 Effects of mean stress on nominal strength of notched polysilicon MEMS structures for a relatively low value of cyclic stress amplitude, $\Delta \sigma=2 \mathrm{GPa}$

Figure 6.4 Plastic-like behavior of amorphous silicon predicted by atomistic calculations;

(a) Deviatoric stress versus deviatoric strain; (b) Pressure versus deviatoric strain. 158

Figure 6.5 Yield surface of linear Drucker-Prager plasticity model. 159

Figure 6.6 Calibration of friction and dilatation angles of linear Drucker-Prager model for amorphous silicon with initial density $\rho=2342 \mathrm{~kg} / \mathrm{m} 3$. 160

Figure 6.7 Geometry of notched polysilicon specimen subjected to cyclic loading. ..... 161

Figure 6.8 (A) Local-global model of a notched polysilicon MEMS specimen. (B) Closeup view of the root of the notch, showing the discrete polycrystalline structure surrounded by homogeneous silicon. 162

Figure 6.9 Residual stress distribution after 500 cycles; (a) $\sigma_{\mathrm{m}}=2.0 \mathrm{GPa}, \Delta \sigma=2.0 \mathrm{GPa}$, (b) $\sigma_{\mathrm{m}}=-3.5 \mathrm{GPa}, \Delta \sigma=2.0 \mathrm{GPa}$. 163

Figure 6.10 Homogeneous notched polycrystalline structure containing a single plastically deforming grain boundary. 164

Figure 6.11 Residual stress distribution in notched polysilicon MEMS specimen after 1000 cycles; (a) $\sigma_{\mathrm{m}}=2.0 \mathrm{GPa}, \Delta \sigma=2.0 \mathrm{GPa}$, (b) $\sigma_{\mathrm{m}}=-3.5 \mathrm{GPa}, \Delta \sigma=2.0 \mathrm{GPa}$ 165

Figure 6.12 Residual stress distribution in notched polysilicon MEMS specimen after 1000 cycles; $\sigma_{\mathrm{m}}=2.0 \mathrm{GPa}, \Delta \sigma=2.0 \mathrm{GPa}\left(\psi=35^{\circ}\right)$. 166

Figure A.1 Black silicon spikes ${ }^{[21]}$ 171

Figure A.2 Etching mechanism of the black silicon method ${ }^{[21]}$ 172

Figure A.3 Sketch of the etching results for polysilicon film using trial recipe-1 175 
Figure A.4 Polysilicon film thicknesses after 20 seconds etching using trial recipe-2. . 177

Figure A.5 Black spots in the trenches after 20secs etching for the polysilicon film (trial recipe-2, photomask 'odd layer').

Figure A.6 Black spots in the trenches after 40sec etching for the polysilicon film (trial recipe-2, photomask 'odd layer').

Figure A.7 Clear trench areas (polysilicon film, trial recipe-3 and photomask 'odd layer').

Figure A.8 Clear trench areas (sputtered silicon film, trial recipe-3 and photomask 'odd layer'). 183

Figure A.9 Top view of the anchor area before etching the sputtered silicon film. 185

Figure A.10 Top view of the anchor area after etching the sputtered silicon film using trial recipe-3 186

Figure A.11 Photoresist thickness vs. spinning speed, Shipley 1813 .......................... 190

Figure A.12 Photoresist thickness vs. spinning speed, AZ 9260 ............................. 190

Figure A.13 Feature profile after filling photoresist in the $7 \mu \mathrm{m}$ wide gap................... 193

Figure A.14 Photoresist filling result after applying photoresist using the parameters shown in Table A.22. 194

Figure A.15 Photoresist filling result using the parameters shown in Table 3.30. 195 


\section{Acknowledgements}

I wish to express my sincere appreciation to Dr. Roberto Ballarini, my dissertation advisor, for his constant support, encouragement and guidance during the completion of my graduate program at Case Western Reserve University.

I gratefully acknowledge the Department of Civil Engineering at Case Western Reserve University. Financial support from the National Science Foundation and the Case School of Engineering's Case Prime Fellowship program is appreciated.

I also wish to thank Dr. Harold Kahn and Dr. Christian Zorman for their helpful advice and suggestions.

Finally, I am especially grateful to my wife and my family, to whom this work is dedicated, for their unconditional support, invaluable encouragement and loving understanding. 


\title{
A Bioinspired Micro-Composite Structure
}

\author{
Abstract \\ by \\ LI CHEN
}

This thesis involves the design, fabrication and mechanical testing of a bioinspired composite structure with characteristic dimensions of the order of tens of microns. The particular microarchitecture, designed and fabricated using microelectromechanical systems (MEMS) technology, involves two distinct length scales and represents a first attempt at mimicking the crossed-lamellar microstructure of the shell of the Giant Queen Conch Strombus gigas, which contains features the dimensions of which span five distinct length scales. After giving a review of the mechanical properties of mollusks, the detailed design of the microstructure, which approximates the crossed-lamellar arrangement of Strombus gigas, is presented. Fabrication of the microstructure using multi-microfabrication methods is conducted in terms of the designed fabrication flow. The problems encountered during the processes are discussed. The measurements of the flexural strength and toughening of the fabricated microstructure are conducted using a commercially available nanoindenter. Testing results are discussed and conclusions about the mechanical behaviors of the microstructure are drawn to summarize the achievement of this thesis. Finally, future work is outlined to point out the possible directions for improving the mechanical performance of the bioinspired composite. 
In parallel with my thesis research, I have developed a theoretical model for the experimentally observed cyclic loading-induced strengthening in MEMS polycrystalline silicon. The model relies on atomistic calculations that predict plastic-like behavior of amorphous silicon, which depending on initial density, is associated with dilatancy or compaction. The amorphous silicon is approximated as a Drucker-Prager plastic material, whose parameters are chosen to match the predictions of the atomistic calculations. The constitutive model is used to simulate the mechanical response to cyclic loads of notched polysilicon MEMS specimens containing deforming amorphous grain boundaries. The results demonstrate that certain combinations of mean stress and alternating stress produce plastic deformation and significant residual compressive stresses at the root of the notch, and in turn an increase in nominal strength. This work is presented in Chapter 6. 


\section{Chapter One}

\section{Introduction}

Through millions of years of evolution, nature has developed various species that possess shell structures. These shell structures can fulfill different biological functions by taking advantage of the extraordinary mechanical, chemical and sensory properties of their constituent materials. They have long been a source of inspiration for the technological development of human beings.

Figure 1.1 shows some examples of creatures with shell structures. Most of the shell structures are protective parts and thereby keep the living tissue away from the harsh environment. The advantageous properties of these protective shells are a result of their complex hierarchical arrangement. Typically, they are composite materials of two or more monolithic materials, and contain structural features spanning numerous distinct length scales.

Careful experimental studies have revealed that most of the tough shell structures are hierarchical nano-composites that undergo a wide variety of energy-absorbing mechanisms spanning various length scales. In the past decades, a large amount of research has been conducted to characterize the superior mechanical properties of mollusk shells ${ }^{[1]-[4]}$. 

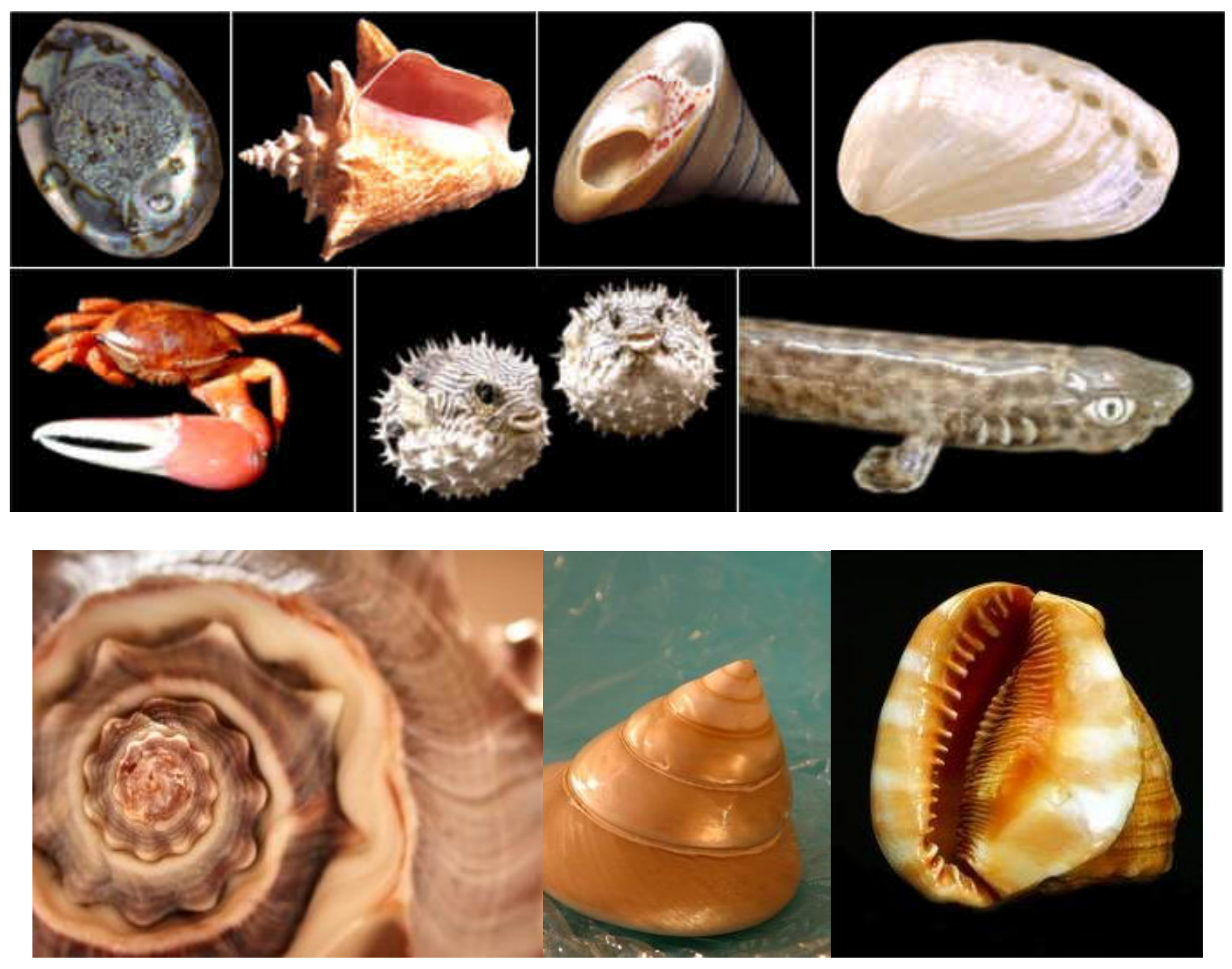

Figure 1.1 Creatures with tough shell structures.

\subsection{Mollusk shells}

Mollusk shells are composed of aligned aragonitic or calcitic calcium carbonate $\left(\mathrm{CaCO}_{3}\right)$ grains separated by extremely thin protein layers. The calcium carbonate grains are arranged at multiple length scales in order to form different microstructures. All mollusk species may possess one or more microstructures, such as columnar nacrearagonite, sheet nacre-aragonite, or crossed-lamellar-aragonite. However, the most 
common microstructure found in mollusk shells is the crossed-lamellar. Figure 1.2 shows the basic arrangement of the crossed-lamellar structure.
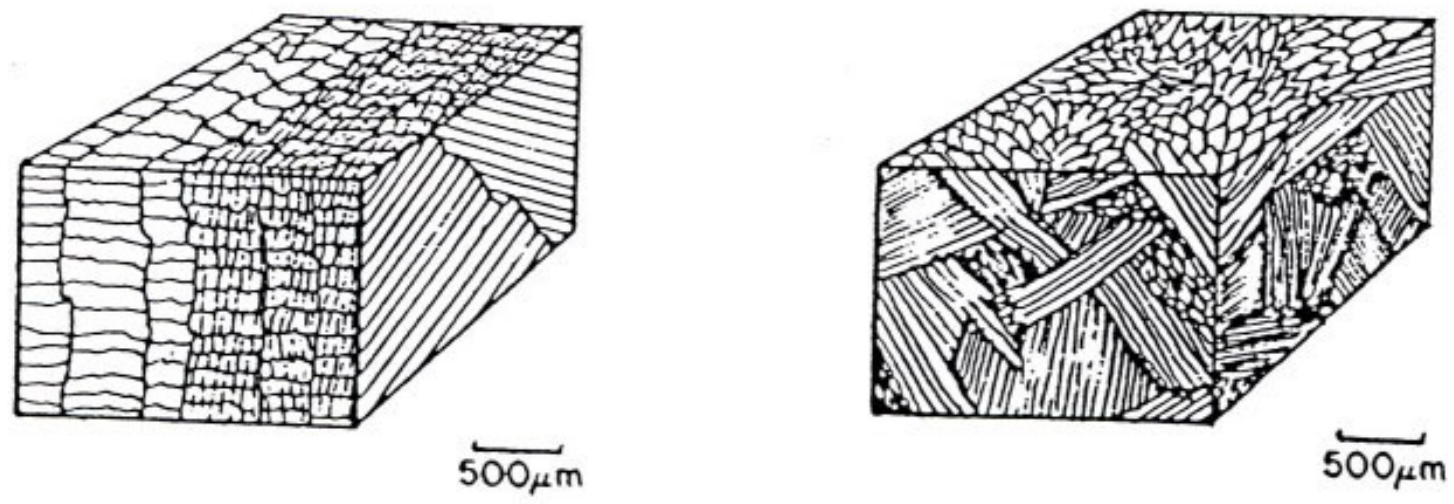

Figure 1.2 Schematic representations of the Crossed-lamellar aragonite.

In most of these mollusk shells, the mineral content comprises up to $96 \%-99 \%$ of the volume of the shell. The rest are organic components such as protein. The tough microstructures are associated with low organic content, generally less than $1 \%$, and a complex arrangement of mineral grains. Even such a small amount of organic component can strongly influence the composite compliance. That is why the modulus of the shells is generally within the region of 30-60GPa, much less than the modulus of the single crystal mineral aragonite, which is about $100 \mathrm{GPa}$. The compressive strengths of the mollusk shells are 150-380GPa--3-7 times higher than their tensile strengths, which regularly lie in the region between $30-130 \mathrm{GPa}{ }^{[1]-[4]}$. This indicates that mollusk shells with high mineral content are naturally brittle.

All of these mechanical characteristics are due to the micro-arrangement, order or orientation of the mineral grains and the organic components. Therefore, a detailed 
description about the shell microstructure is necessary in order to understand its mechanical behavior. Kuhn-Spearing et al. have provided a good understanding of the micro-architecture of Strombus gigas, the Queen Conch native to Caribbean habitats ${ }^{[7][8]}$. They also made specimens from this conch shell to measure the fracture toughness of the shell. Kamat et al developed mechanics models to explain the deformation mechanism of the shell structure ${ }^{[9]}$. These works have shown the details of the microstructures on the nanometer scale, and pointed out that the high toughness is directly related to the complex hierarchical microstructure.

\subsection{Strombus gigas}

\subsubsection{Microstructure}

Strombus gigas, as shown in Figure 1.3, is one of the largest in the conch family. By observing a cross-sectional micrograph of a fractured shell, five length scales can be found in the microstructure. The five length scales are the macroscopic layer, the firstorder lamella, the second-order lamella, the third-order lamella, and twins within each

third-order lamella ${ }^{[6]}$. The basic building blocks are the third-order lamellae, each of which is layered at an atomic scale by very fine-growth polysynthetic twins. Many parallel third-order lamellae form one second-order lamella. The second-order lamellae are embedded within each first-order lamella. Each macroscopic layer is composed of first-order lamellae, which are parallel to each other in one macroscopic layer, but change their orientation by $90^{\circ}$ in the adjacent macroscopic layer. Therefore, the build up is in a $0^{0} / 90^{\circ} / 0^{0}$ arrangement. It is schematically depicted in Figure 1.4(a). Figure 1.4(b)-(d) are 
SEM images of a fracture surface showing a cross-section of the crossed lamellar structure taken from a specimen of Strombus gigas. As shown in Figure 1.4(a), the macroscopic layer is labeled as inner layer, middle layer or outer layer, based on its relative location in the lip area of the shell.

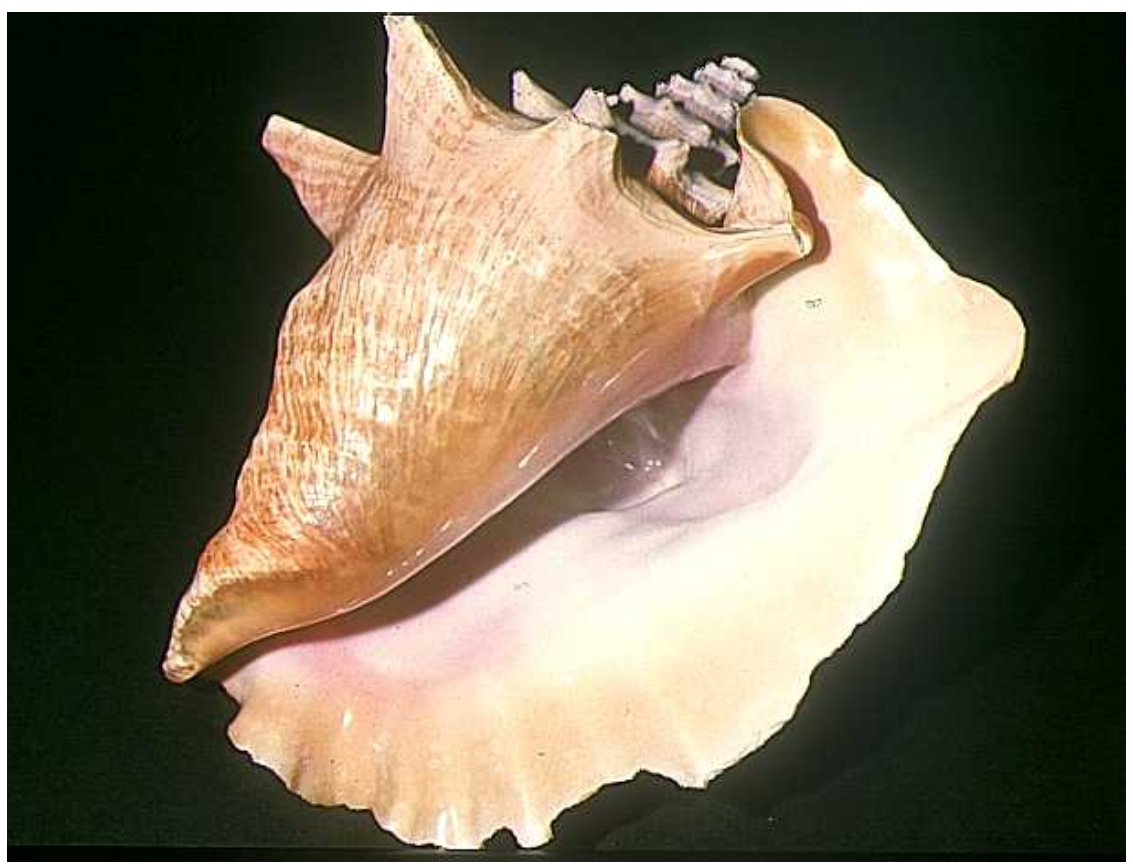

Figure 1.3 Strombus gigas. 


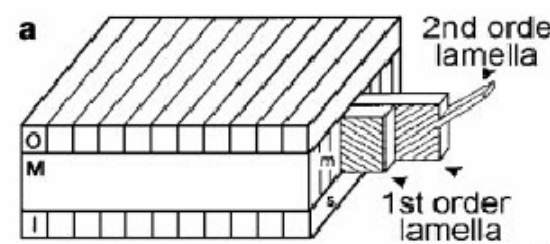

Layers: $0.5-2 \mathrm{~mm}$ thick

First order lamellae

5-60 $\mu \mathrm{m}$ thick; many $\mu \mathrm{m}$ wide
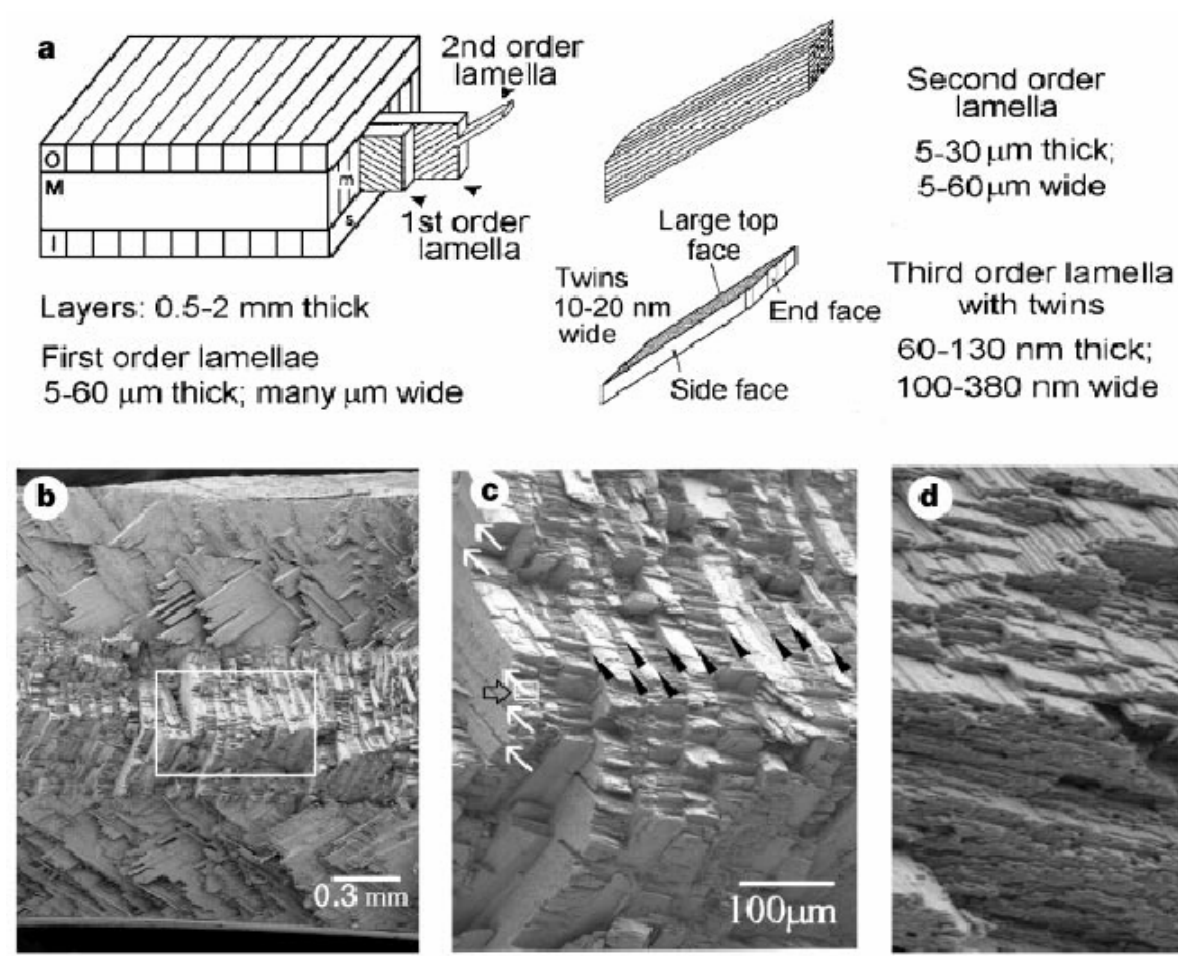

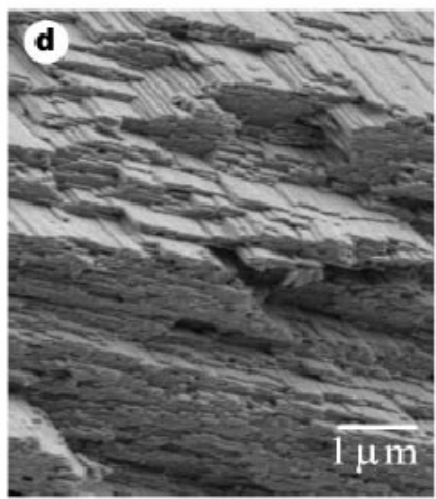

Figure 1.4 (a) - A schematic representation of the crossed lamellar microstructure of the Strombus gigas. (b), (c) and (d) - SEM images of the fracture surface of a bend specimen taken at increasing magnification. (O, M and I refer to outer, middle and inner layers) ${ }^{[6]}$.

The organic material surrounds the third order lamella, the second-order lamella, the first-order lamella and the macroscopic layer interface. Figure 1.5(a) is a SEM micrograph of a polished and partially demineralized sample of Strombus gigas. The C-C line in the micrograph is the boundary separating adjacent second-order lamellae within individual first order lamella, while the $\mathrm{BCCB}$ line and the $\mathrm{BCB}$ line separate adjacent first order lamellae. Figure 1.5(d) is a TEM micrograph showing the third-order lamellae 'end-on'. The most obvious features of the third-order interfaces are the organic matrix particles distributed along the largest face of the third-order lamellae. In fact, a membrane-like organic matrix is continuous around each third-order lamella. These images confirm that the organic matrix is present at every interface. 

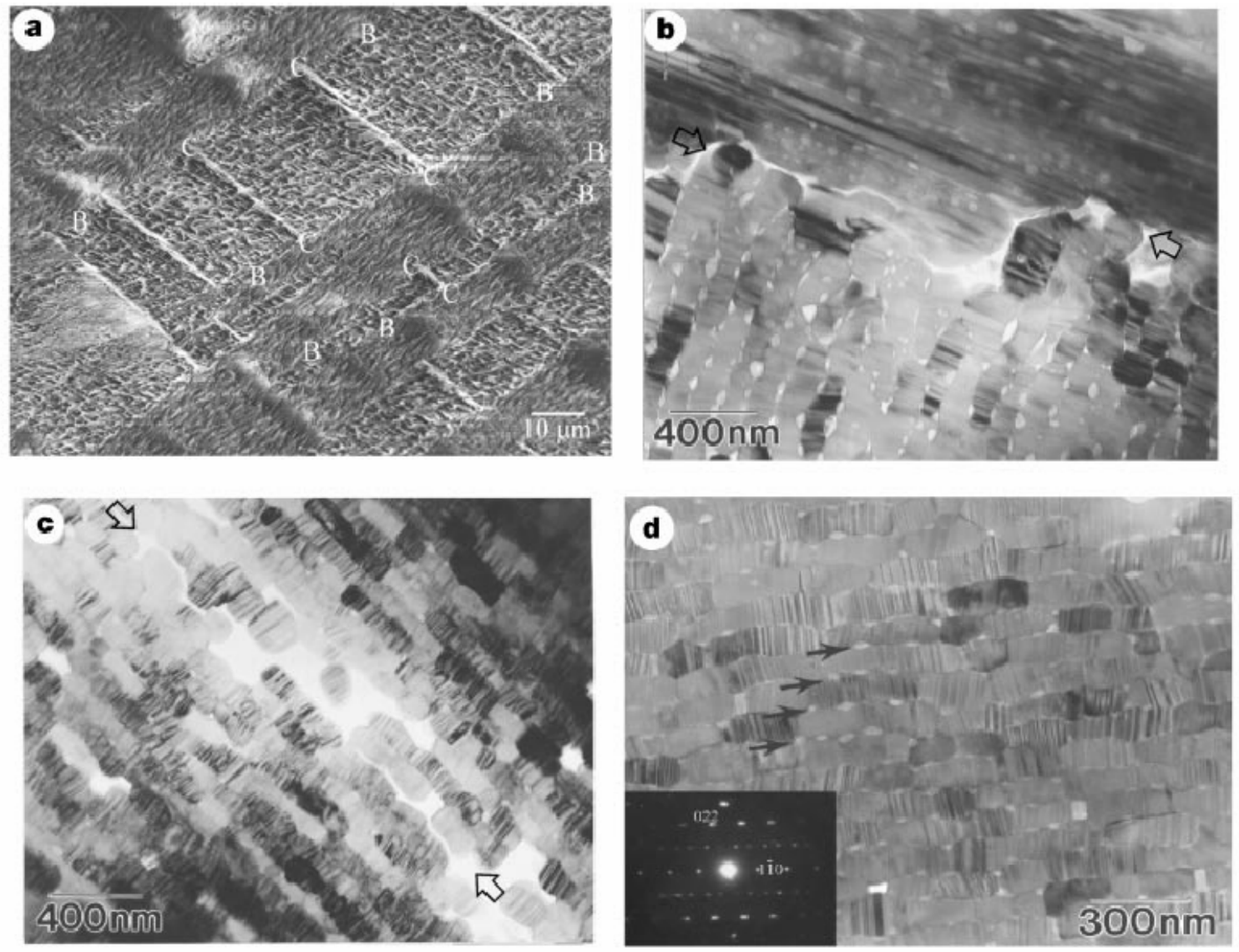

Figure 1.5 (a) SEM image of the interfaces separating second-order lamellae (CC) and the interfaces separating first-order lamellae (BCCB and BCB). (b) TEM image of a firstorder interface. (c) TEM image of a second-order interface. (d) TEM image of a thirdorder lamellae 'end-on' ${ }^{[6]}$.

\subsubsection{Mechanical properties}

Previous studies on the Strombus gigas shell have shown that they have remarkable toughness and work of fracture. Most of the mechanical properties of the shell of Strombus gigas have been measured using specimens made from the thicker lip section of the shell. Kuhn-Spearing et al. conducted four-point bending tests on unnotched specimens ${ }^{[7]}$. The tested specimens were polished to show the crack front as 
sketched in Figure 1.6. This experiment clearly showed tunneling cracks whose density increased within the inner layer as the load was increased. Thereafter, a saturation tunneling crack density was reached, and in turn, one or more tunneling cracks started to grow through the middle layer, along the interfaces between second-order lamellae.

The reason why the cracks did not propagate catastrophically through the middle layer is that the adjacent first-order lamellae retarded their growth through bridging forces. This so-called bridging mechanism is the direct explanation for the extremely high work of fracture. Therefore, multi-tunnel-cracking and crack bridging are the two main energy-dissipating mechanisms in the shell.

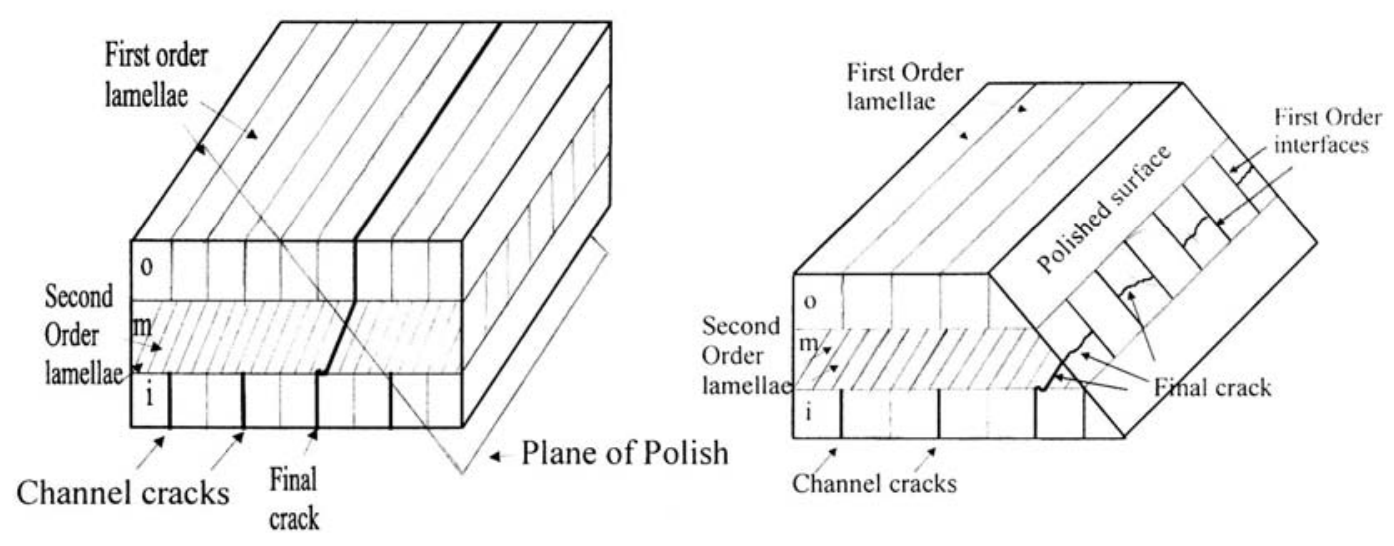

Figure 1.6 Schematic drawing of the micro-crack fronts ${ }^{[5]}$.

Kamat et al. tested notched specimens using four-point bending method to investigate the fracture toughness and the work of fracture of the shell ${ }^{[5]}$. Two kinds of specimens were prepared as sketched in Figure 1.7. One had the notch initiated from the 
inner layer, and extended to the middle layer. The other one had the notch introduced from the middle layer, and extended to the inner layer.

The fracture toughness of the tough middle layer was about $2.2 \mathrm{MPa} \cdot \mathrm{m}^{1 / 2}$, four times as large as that of the weak inner layer. The work of fracture is defined as the area under the load-displacement curve divided by the fracture surface area. As seen in Figure 1.7, the tough middle layer had much larger fracture load and much bigger area under the load-displacement curve. Experimental data showed that the work of fracture of the tough middle layer was $0.325 \mathrm{KJ} \cdot \mathrm{m}^{-2}$, almost an order of magnitude difference from the value of the weak inner layer and 100-1000 times as large as the value of the non-biogenic $\mathrm{CaCO}_{3}$.

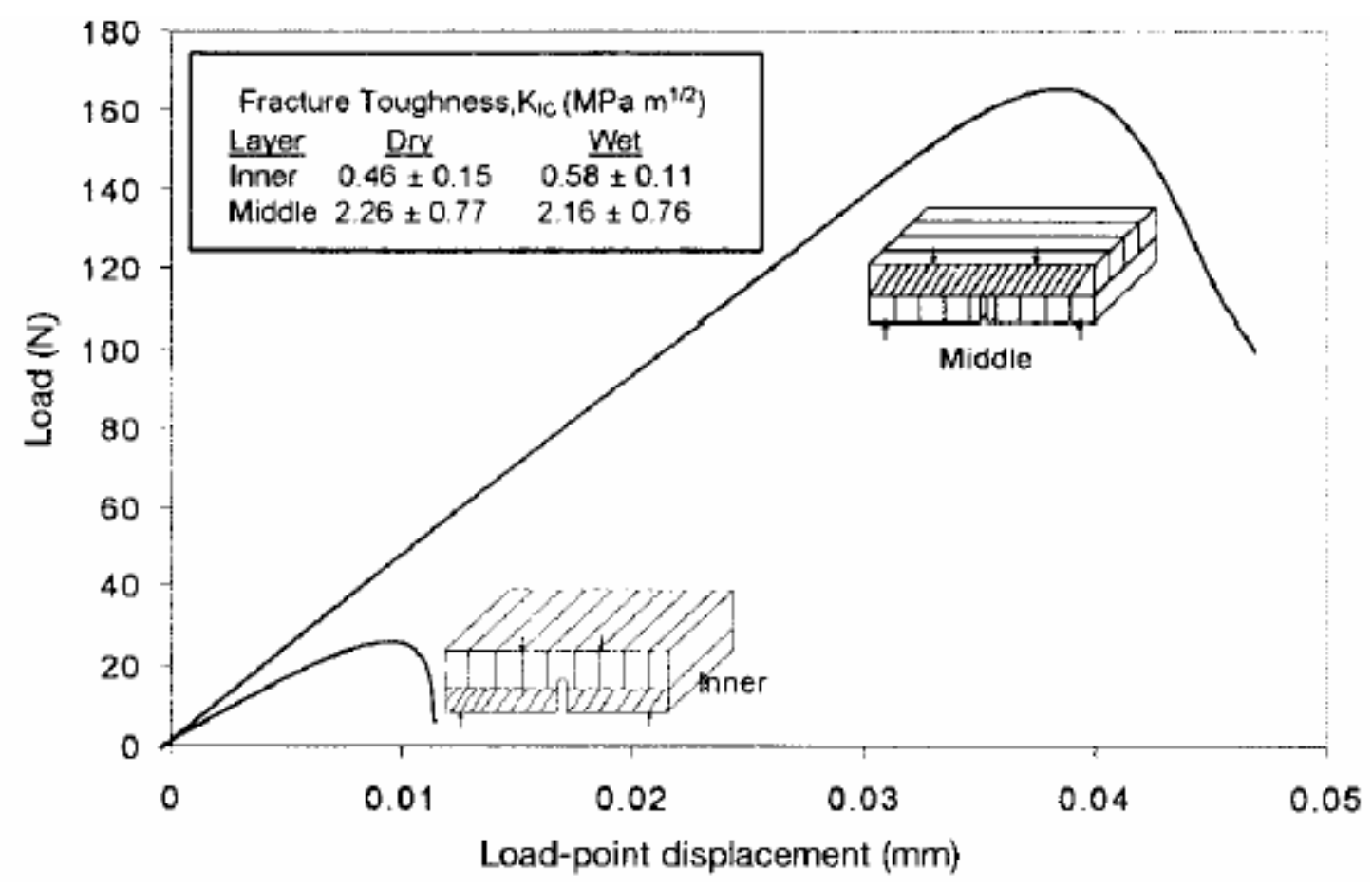

Figure 1.7 Load-Displacement curves for tough middle layer and weak inner layer specimens ${ }^{[5]}$.

All of the remarkable mechanical properties of the conch Strombus gigas ensure its important role in guiding the biomimetic design of tough, lightweight structures. This 
thesis is inspired by the exquisitely arranged microstructure and attempts to duplicate the micro-architecture in order to produce the same energy-dissipating mechanism.

\subsection{Biomimetics—synthetic multi-layer materials}

After recognizing the tremendously outstanding mechanical characteristics of the conch shell, researchers started searching for feasible fabrication methods to duplicate such microstructures. The challenges include the following:

1. Feasible fabrication procedures have to be developed to build a multi-layer structure.

2. Order and orientation controls are critical for successful duplication. The order level within micrometer scale can be made without technical difficulties. However, the order level with nanometer length scale is difficult to achieve with the currently available fabrication facilities.

3. Much effort has to be made on finding organic-like materials to simulate the organic component between different order layers, and further, to develop a way to apply the organic-like materials in the microstructure.

4. Proper testing methods are also important for verifying the synthetic microstructure. This involves designing specimens to fit fabrication capabilities, finding test instruments with ideal resolutions, and eventually developing the whole process flow.

Since the extremely high toughness is a result of the complex micro-architecture of the conch shell, it is clear that the same multi-layer arrangement with the proper order level and layer orientation has to be duplicated so that high toughness can be obtained. 
Because the multi-layer microstructure is difficult to fabricate, most researchers begin their attempts from simply arranged multi-layer structures. The simplest approach is to coat soft materials on the surfaces of thin plates that are made by hard materials, and then stack the coated thin plates up to form a multi-layer structure with weak interfaces.

Clegg et al. ${ }^{[1]]}$ took advantage of this approach to make tough ceramics. They made silicon carbide powder into thin sheets and coated these sheets with graphite to form weak interfaces. Then these thin sheets were pressed together and sintered without pressure. The flat sheets were $50 \mathrm{~mm} \cdot 50 \mathrm{~mm}$ squares with a thickness of 200 microns. The final stacked structure was about $2 \mathrm{~mm}$ thick. Testing specimens were made by cutting the stacked structure into $2 \mathrm{~mm}$ wide strips and introducing notches in the middle of the specimens. A sketch of the $\mathrm{SiC} /$ graphite structure is shown in Figure 1.8.

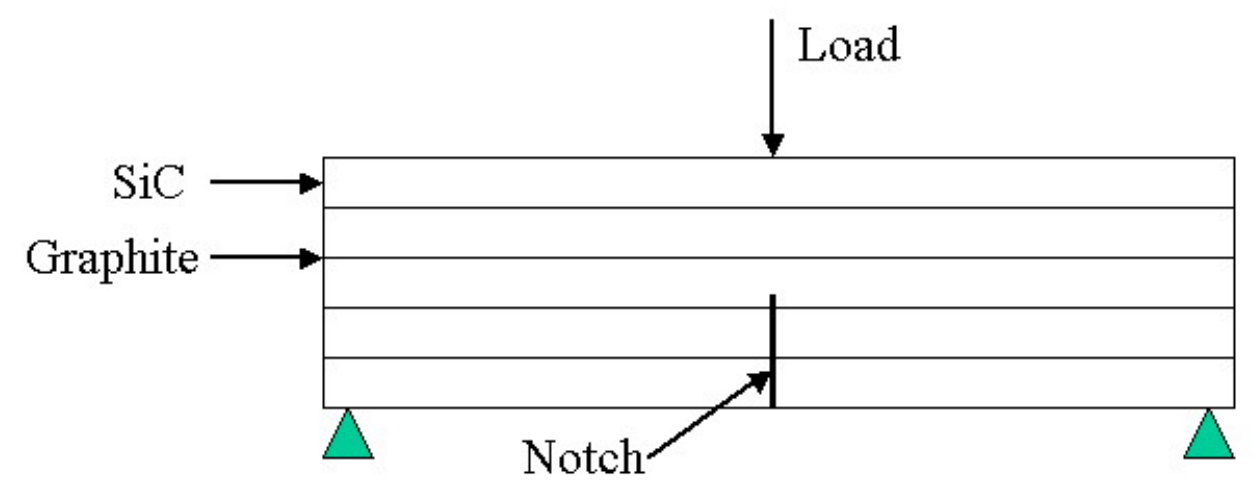

Figure 1.8 Sketch of the SiC/graphite structure.

Fracture toughness of the notched specimens was tested using the three-point bending method. A typical load-deflection curve is shown in Figure 1.9. The deformation of the laminated structure was linear elastic before point A on the curve. Thereafter, the structure deformed plastically. After the load-deflection curve reached point B, a crack 
was initiated. Once the crack propagated to the layer interface, it was deflected by the interface and branched along the interface or through the $\mathrm{SiC}$ layer. The branching cracks propagated and caused part of the sample to break away. The situation after the branching cracks traveled through several layers is shown in Figure 1.10. In Figure 1.9, where each drop in the jagged load-deflection curve corresponds to one or more cracks generated through the $\mathrm{SiC}$ layer or along the interface. Even though the structure cannot support higher load after reaching the critical point, it will still not fail catastrophically. Compared to the monolithic silicon carbide sample, the multi-layer structure achieves a fourfold increase in fracture toughness and more than a hundredfold increase in work of fracture.

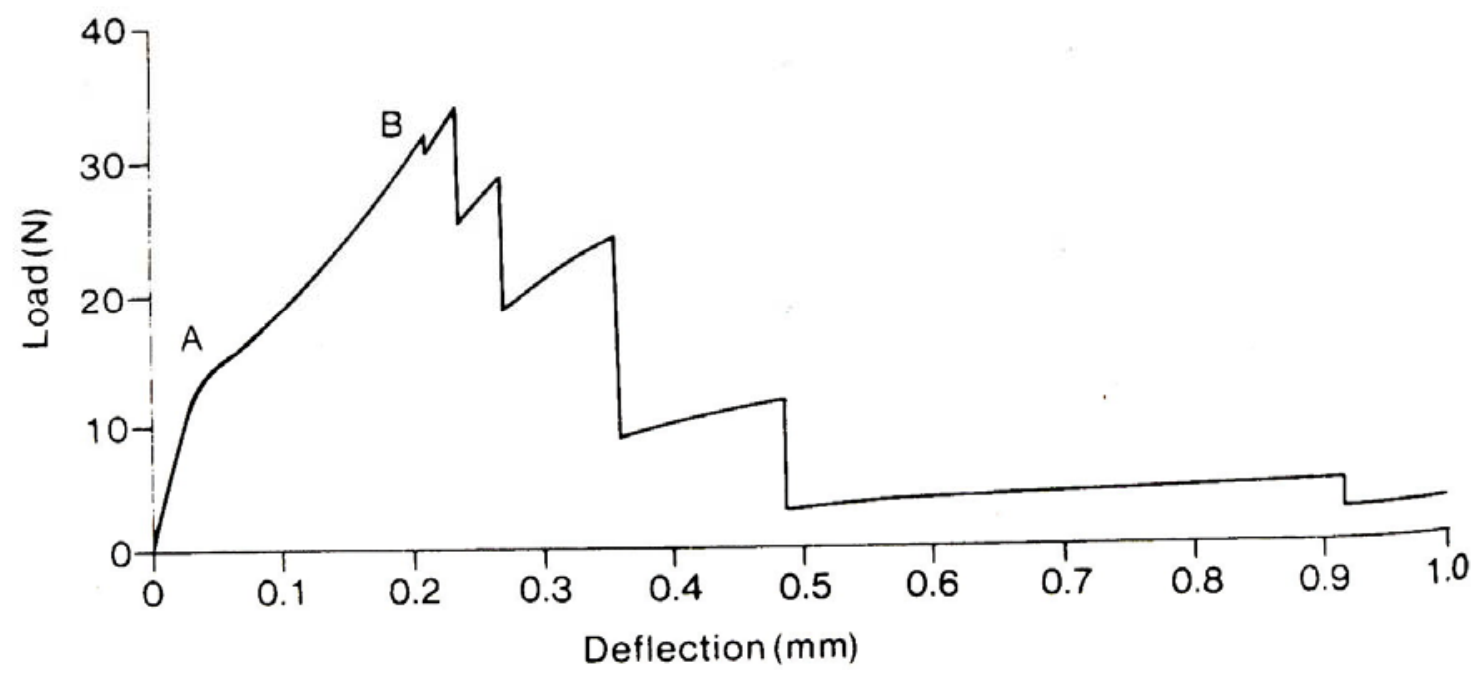

Figure 1.9 Load-deflection curve of a notched SiC/graphite structure ${ }^{[11]}$. 


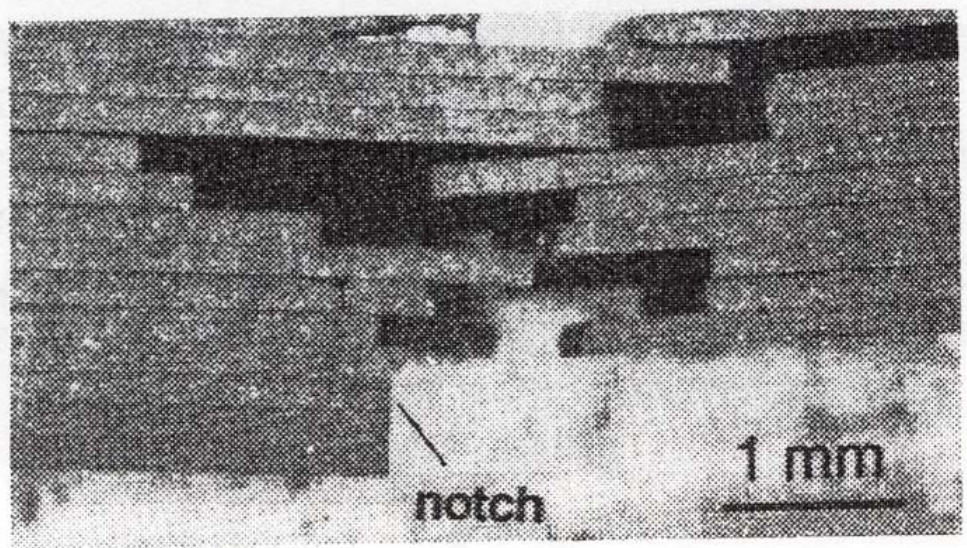

Figure 1.10 Branching cracks travel through several layers ${ }^{[11]}$.

More complicated fabrication approaches were used by Calvert et al. to synthesize multilayered systems. Employing the successful experience Clegg et al. gained, Calvert first made a six-layer epoxy structure with fiberglass mesh as interface material. Figure 1.11 presents the comparison of load-deflection curves for six-layer epoxy without interlayer, with one interlayer and with five interlayers. The structure with five interlayers exhibits extended load-deflection curve and slowly reaches the catastrophic failure. 


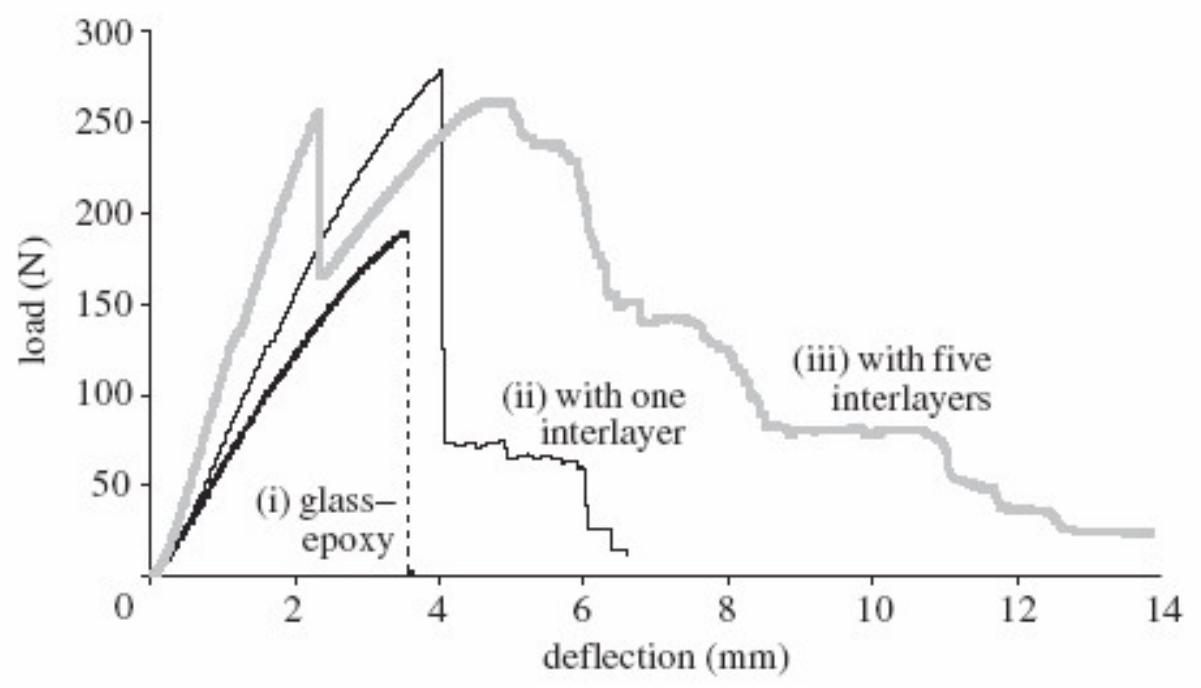

Figure 1.11 Load-deflection curves for six-layer epoxy without interlayer, with one interlayer and with five interlayers ${ }^{[10]}$.

In order to satisfy the requirement of high temperature application, they used metals as interface materials to fabricate tough ceramics. A porous ceramic was made and infiltrated with a liquid metal. The porous ceramic was made of alumina with dense ceramic surface, dense sides and a gradient of interconnected porosity. After infiltrated with the liquid metal, the composite had one aluminum surface and one ceramic surface. Cross-sections of the sample composite parts are shown in Figure 1.12. 
(a)

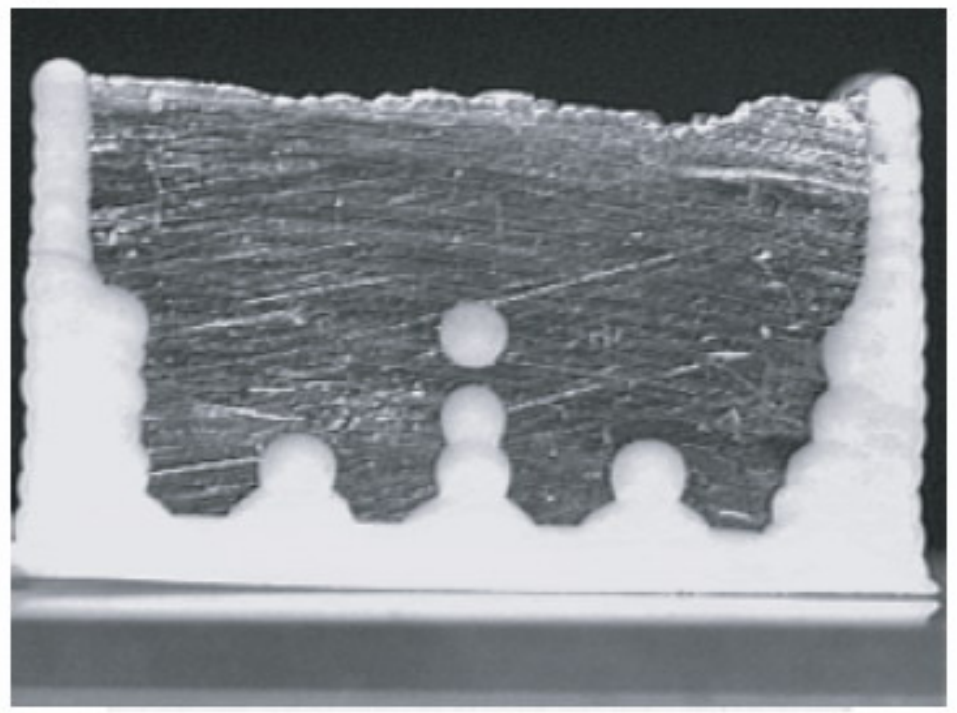

(b)

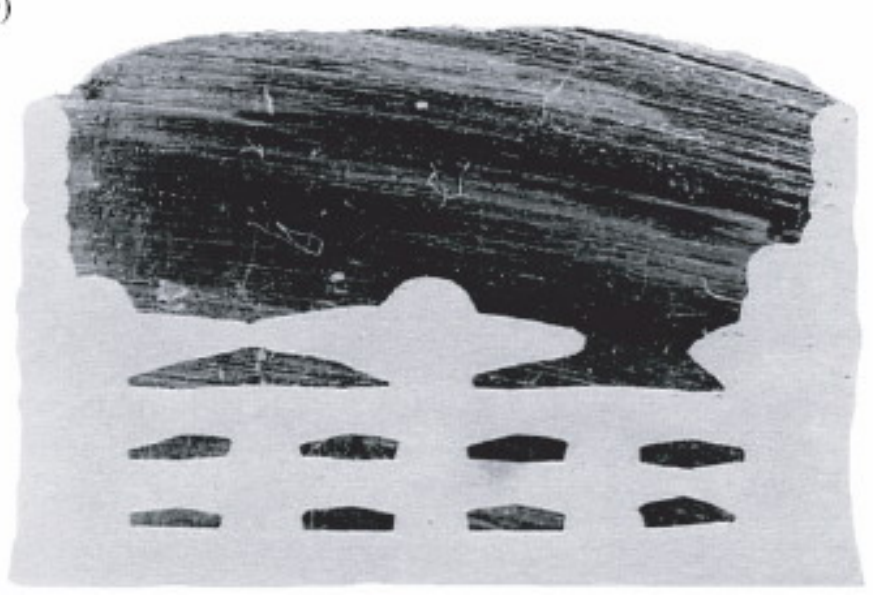

Figure 1.12 Cross-section of alumina/aluminum composite parts made by free-forming porous ceramic and backfilling with metal. (a) Section through pore region. (b) Section through ceramic crossbars ${ }^{[10]}$.

Typical load-deflection curves for the composites under a four-point bend loading with the ceramics surface in tension are presented in Figure 1.13. The jagged curves prove that the cracks are deflected by the metal layer. 


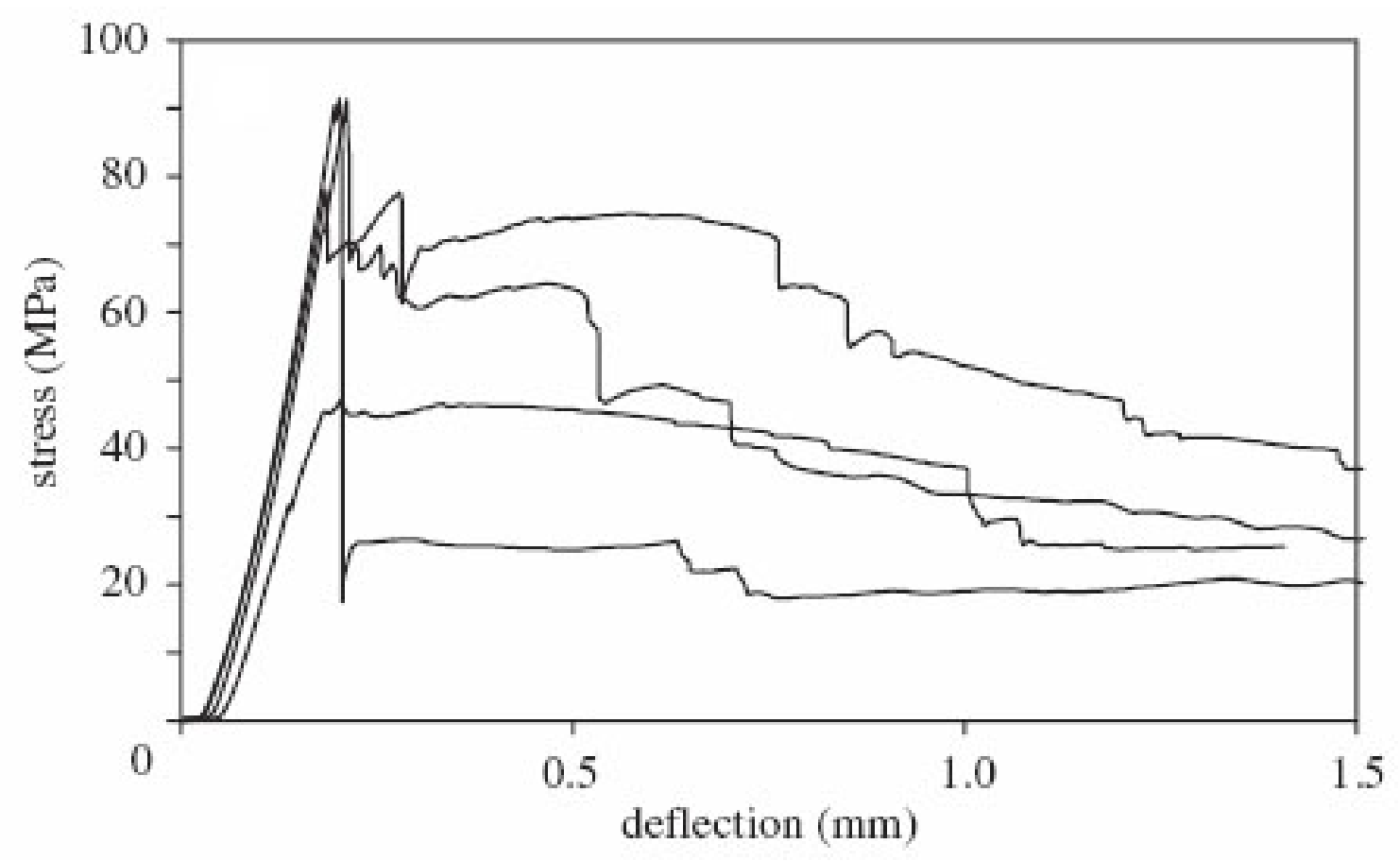

Figure 1.13 Load-deflection curves for alumina-aluminum composites under a four-point bend loading with the ceramic surface in tension ${ }^{[10]}$.

All of the research described above achieved successful results in the sense of duplicating the crack-stopping mechanism. These synthetic composite structures are all in the macro-scale with the deflection in the millimeter range. So far, no micro-composite structure has been fabricated and studied. This thesis will present the first attempt to synthesize the micro-composite structure and study its mechanical properties.

\subsection{Thesis outline}

This thesis, which deals with the fabrication of a micro-composite structure and the testing of its mechanical properties, is structured as follows. Chapter 2 introduces suitable materials used as components of the microstructure and available facilities for the micro-fabrication. Based on the comparison of their advantages and disadvantages, 
appropriate material combination and fabrication methods are chosen. In Chapter 3, detailed fabrication procedures are given and all the problems encountered during the micro-fabrication are discussed. Some mechanical properties of the structural components in the form of thin films are tested. Detailed reports about the testing results are also presented in this chapter. Chapter 4 introduces the nanoindenter and its operation procedures for the vertical and lateral testing. In Chapter 5, testing results are presented and discussed. Conclusions about the mechanical behavior of the micro-composite structure are drawn to summarize the research exhibited in this thesis. Some future works are also outlined in this chapter to point out the possible approaches for improving the synthetic microstructure. Chapter 6 presents a theoretical model developed for explaining the cyclic loading-induced strengthening in MEMS polysilicon devices. The simulation results are compared with the experimentally measured data. 


\section{Chapter Two}

\section{Design}

The main goal of this thesis is to mimic the microstructure of the shell of the conch Strombus gigas with appropriate material combination (brittle and ductile materials). Two questions arise regarding this issue. What materials can be used in the synthetic microstructure to approximate the shell components? How can currently available facilities be used to fabricate the microstructure with the selected materials? In order to answer these two questions, a material survey and a facility survey must be conducted.

\subsection{Available Materials}

In order to duplicate the microstructure of the Strombus gigas shell, appropriate material combination has to be found to replace the shell components (calcium carbonate and protein). What determines whether significant tunneling cracking, crack surface bridging, and crack deflection occur is the ratio of toughness between the mineral phase and the organic matrix. The ratio of elastic modulus, which is about 100 for Strombus gigas, is not important. However, the materials available to us turn out to have roughly the same stiffness ratios.

One choice is to use silicon carbide and graphitic carbon (SiC/C). Clegg et al. had already used $\mathrm{SiC} / \mathrm{C}$ to fabricate a composite structure and obtained a fourfold increase in the fracture toughness with respect to the monolithic SiC. Silicon carbide does not react with graphite even at very high temperature. Another advantage of $\mathrm{SiC} / \mathrm{C}$ is that a very 
thin graphite layer can be coated between the $\mathrm{SiC}$ sheets, thus the volume ratio of the components in the synthetic microstructure can be very close to that in the shell structure.

Another option is to use silicon and photoresist. Silicon is a commonly-used material in the IC industry. Many mature microfabrication methods have been developed for silicon-related processes. Photoresist is a polymer. It is widely used in the IC industry for transferring features from photomasks to silicon wafers. Photoresist can be transferred to a carbon-like material after being baked at the temperature of $115{ }^{0} \mathrm{C} \sim 160{ }^{0} \mathrm{C}$. Silicon and photoresist will not react with each other. However, photoresist cannot sustain high temperature (over $200{ }^{\circ} \mathrm{C}$ ) and this will significantly affect its application in the synthetic microstructure. Moreover, currently available facilities can generate 1 15 microns thick photoresist film, which is not very thin compared to the silicon film. Thus, high volume ratio cannot be obtained for silicon/photoresist.

\subsection{Facility survey}

The synthetic microstructure was fabricated in Micro-Fabrication-Lab (MFL), at Case Western Reserve University. The feasibility analysis is based on a survey of the available facilities in MFL.

Successful fabrication of the synthetic microstructure involves two major tasks: deposition of the thin film material and feature transfer in the deposited thin film. For the silicon/photoresist, all the processes can be performed in the MFL with currently available facilities. The only problem is that photoresist cannot bear temperature that is higher than $150{ }^{\circ} \mathrm{C}$. A low temperature process has to be used to grow a thin silicon film after the first photoresist application. Sputtering is an available method for such a low 
temperature requirement. Another challenge is the photoresist application in the patterned silicon film. In this step, photoresist will be filled into the gaps among the silicon features using a spinner. Since this process is a physical operation, gaps in the interface of silicon and photoresist are inevitable. This will significantly lower the quality of the synthetic microstructure.

If only mechanical properties of the synthetic microstructure are considered, $\mathrm{SiC} / \mathrm{C}$ has significant advantages over silicon/photoresist. The $\mathrm{SiC} / \mathrm{C}$ is more difficult to realize because currently available tools in the MFL cannot deposit graphite carbon. Some of the reactors in the MFL can produce carbon as byproduct or can obtain carbon as target material after adjusting tool parameters and supply gases. However, this will cost more time and money to maintain the tools since carbon will contaminate the reactor chambers and keep causing problems in other fabrication tasks.

Thus, assembling a new reactor especially for depositing graphite carbon is necessary. The positive side of building a new reactor is that the quality of the carbon film is guaranteed by optimizing the gas supplies and controlling the reactor chamber parameters. In MFL, a used Plasma Enhanced Chemical Vapor Deposition (PECVD) reactor is appropriate for this goal, and graphite carbon can be one of the target materials by designing a process recipe and using propane and hydrogen as reaction gases.

Another option is to use a sputtering tool to deposit the carbon film. Sputtered carbon film with good quality was obtained by Hudek et al. ${ }^{[13]}$. The sketch of the sputtering tool they used is shown in Figure 2.1. In order to get stress-compensated carbon film, Hudek et al. used commercially available equipment to provide thermal treatment. Thus, carbon films with very low stress $(<5 \mathrm{Mpa})$ can be obtained and further 
patterned using RIE with oxygen plasma. Figure 2.2 shows some patterns etched in a low stress film. With this deposition method, the thickness of carbon film can reach 7 microns and the anisotropic etching aspect ratio can be as high as 50 70.

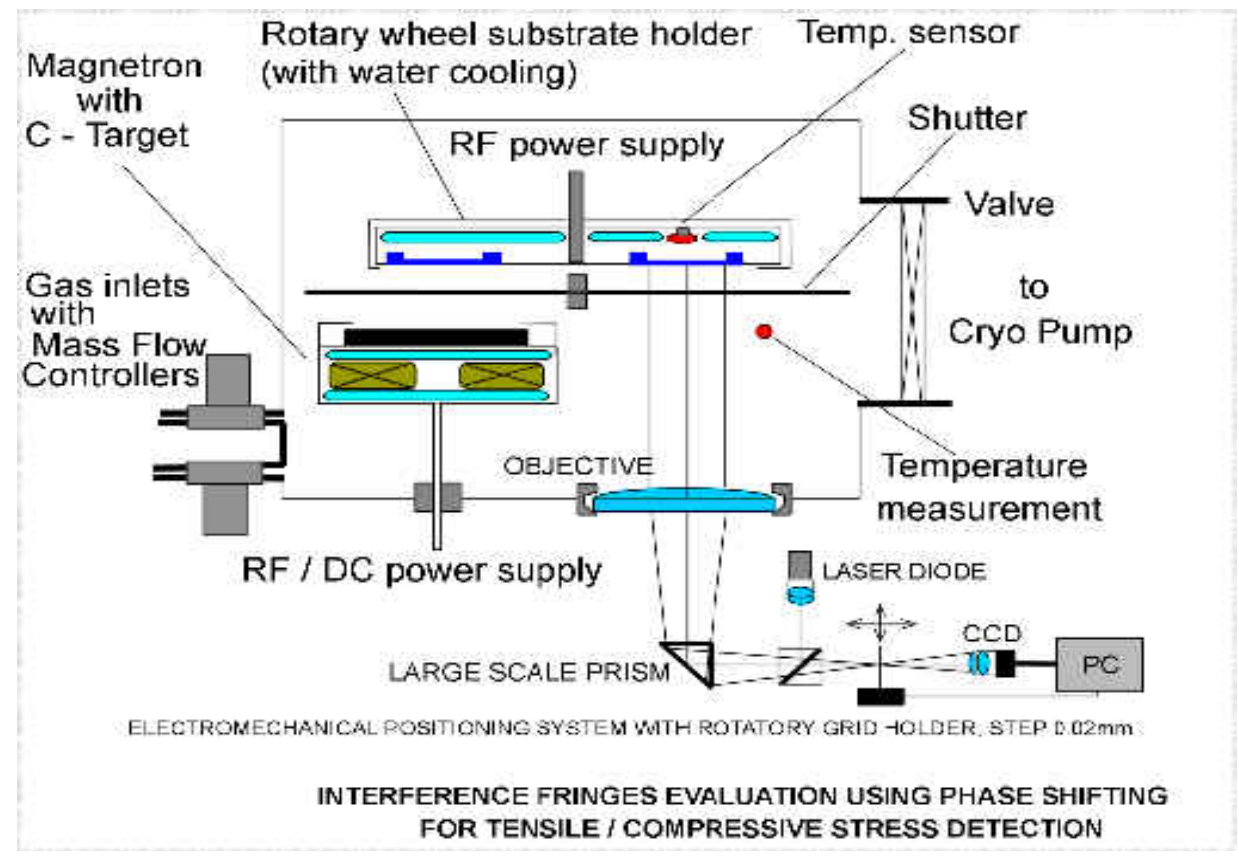

Figure 2.1 Sketch of sputtering tool used by Hudek et al. ${ }^{[13]}$ 


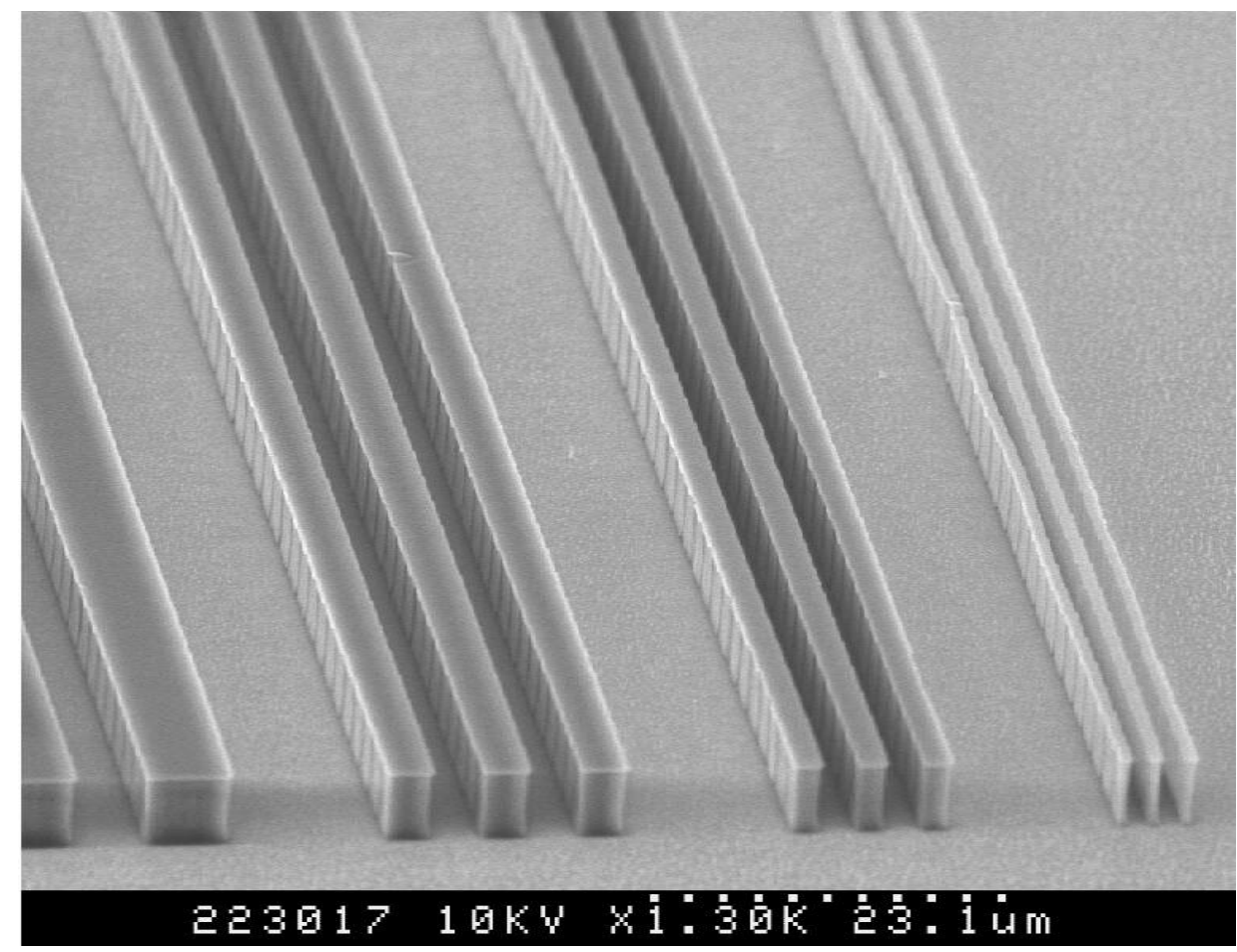

Figure 2.2 Patterns in a carbon film with low stress ${ }^{[13]}$.

Compared to the carbon deposition, silicon carbide deposition has been studied for over eight years at CASE, and SiC film with good uniformity has been successfully grown with an APCVD system ${ }^{[14]-[17]}$. Figure 2.3 shows the sketch of the APCVD system. 


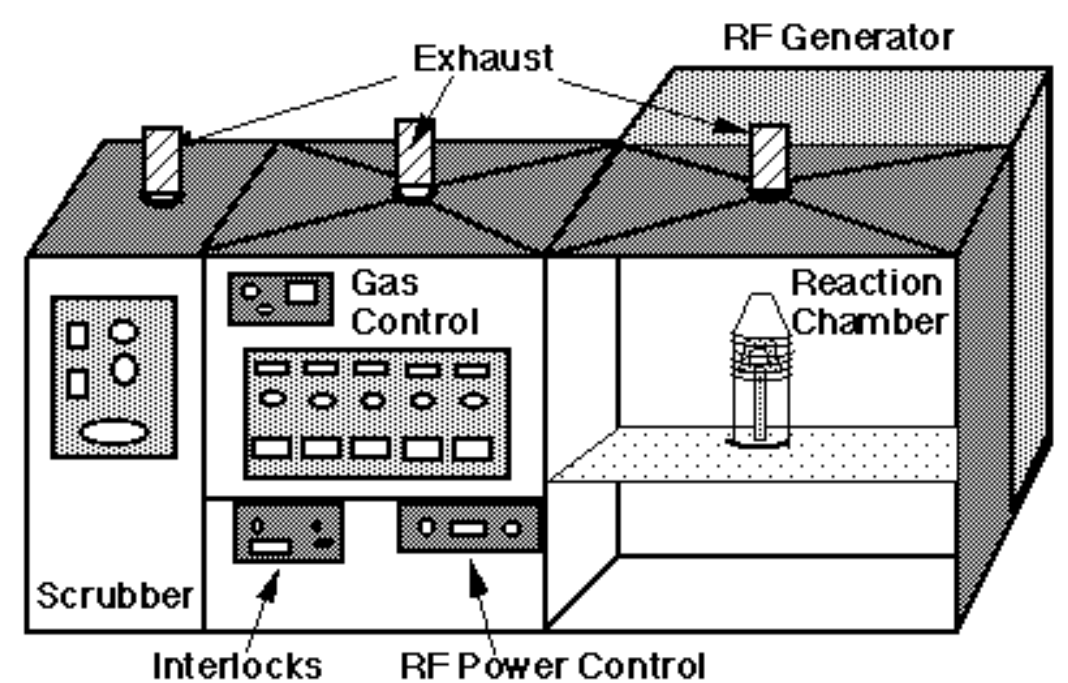

Figure 2.3 APCVD systems for SiC growth.

The reaction chamber is a double-wall quartz tube with an inner diameter of about $170 \mathrm{~mm}$. A SiC-coated graphite susceptor is used to hold two four-inch diameter substrates. A $50 \mathrm{~kW}$ RF generator with a ten-turn induction coil is used to heat the susceptor. The process gases are propane (15\% hydrogen) and silane (5\% in hydrogen). Ultra-high-purify hydrogen, further purified by a hydrogen purification system, is used as the carrier gas. Argon is used as the purge gas since nitrogen is a donor impurity in SiC. The system is capable of depositing SiC films on silicon substrate with deposition rates averaging 166 angstrom per minute at $1280{ }^{\circ} \mathrm{C}$. At a temperature of $1160{ }^{\circ} \mathrm{C}$, this system can grow $\mathrm{SiC}$ films on silicon dioxide substrate without causing any delamination. A reactive ion etching method can be used to etch the $\mathrm{SiC}$ film. This is done with Tegal 803 using $\mathrm{CHF}_{3} / \mathrm{O}_{2}$ at pressure of 1.75 Torr. A maximum $\mathrm{SiC}$ etch rate of $1267 \AA / \mathrm{min}$ can be achieved with a $\mathrm{CHF}_{3} / \mathrm{O}_{2} / \mathrm{He}$ mixture.

The advantages and the disadvantages of $\mathrm{SiC} / \mathrm{C}$ and $\mathrm{Si} /$ photoresist are summarized as follow: 
- $\mathrm{SiC} / \mathrm{C}$, advantages:

1. SiC deposition is robust using the APCVD reactor.

2. No large amount of gaps or defects exists in the interface between SiC and $\mathrm{C}$ due to the nature of thin film deposition.

- $\mathrm{SiC} / \mathrm{C}$, disadvantages:

1. Carbon deposition has not been conducted in the MFL. The quality of the Carbon film is unsure. A large amount of time and a considerable budget are needed to make the deposition of the carbon film successful.

2. SiC film has been successfully grown only on silicon and silicon dioxide substrate. The quality of $\mathrm{SiC}$ film on carbon film is unsure.

3. Only less than 3 microns thick $\mathrm{SiC}$ film can be successfully etched in MFL. Since the depth of the synthetic microstructure will roughly be 10 microns, extra work has to be done for etching thick SiC film.

- Si/Photoresist, advantages:

1. The deposition processes for silicon and photoresist are standard; no extra facility adjustments are required.

2. Dry photoresist has similar mechanical properties as natural protein, so it is a good replacement for soft material in shell structures.

3. Compared to $\mathrm{SiC}$, thick silicon film ( $>10$ microns) can be etched using Reactive Ion Etch (RIE) method with modified etching recipe.

- Si/Photoresist, disadvantages:

1. Gaps or defects are expected to appear in the interface between silicon and photoresist. 
2. Photoresist cannot maintain a solid shape and mechanical properties at temperature higher than $150{ }^{\circ} \mathrm{C}$. Thus, silicon films have to be deposited at low temperature with the sputtering tool.

3. Due to the nature of sputtering process and the low surface hardness of photoresist, the bombarding particles will damage the photoresist structure and further cause more defects in the synthetic microstructure.

Based on the evaluation of the process difficulties and the research budget, $\mathrm{Si} /$ Photoresist was chosen as the first attempt.

\subsection{Design of the synthetic microstructure}

\subsubsection{Fabrication design}

The basic idea for synthesizing the micro-composite structure is to deposit a silicon thin film as the first structure layer, and then dig trenches in this thin film using RIE etching method to create interface spacing. The trenches have the same arrangement as the interfaces between the first-order lamellae in the inner layer and the interfaces between the second-order lamellae in the middle layer of the Strombus gigas shell. Thereafter, photoresist will be filled in the trenches. The same processes will be repeated several times to form multi-layer microstructure. The phrases "inner layer" and "middle layer" will be also used to represent the components in the synthetic microstructure, which simulate the inner layer and the middle layer in the Strombus gigas shell. In the even layers, the direction of the trench will rotate $90^{\circ}$ with respect to the trench direction 
in the adjacent odd layers. Ideal sample areas are covered by etching masks and the uncovered silicon is etched away. Finally, the samples are released and suspend over the wafer substrate with the support of silicon dioxide anchors. Figure 2.4 illuminates the design idea with 3-D sketches on the left and 2-D profiles on the right. This design only simulates the first and the second length-scale in the shell structure. This reflects the fabrication limitation since the fabrication of higher order length-scale layers is beyond the capability of currently available process techniques. 

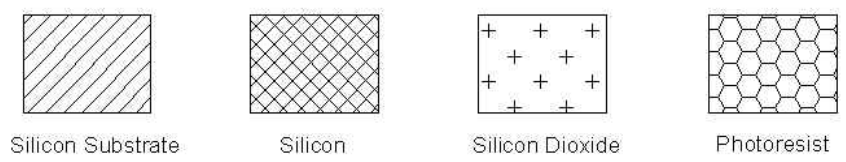

(0) Hatch labels.
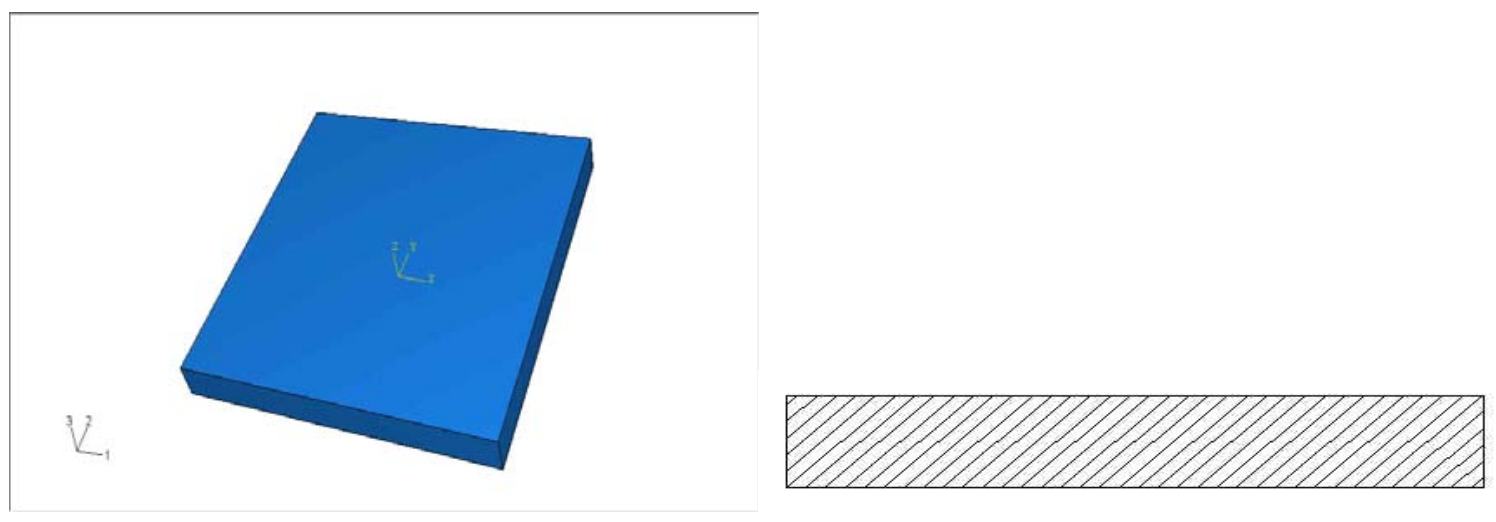

(1) Silicon substrate.
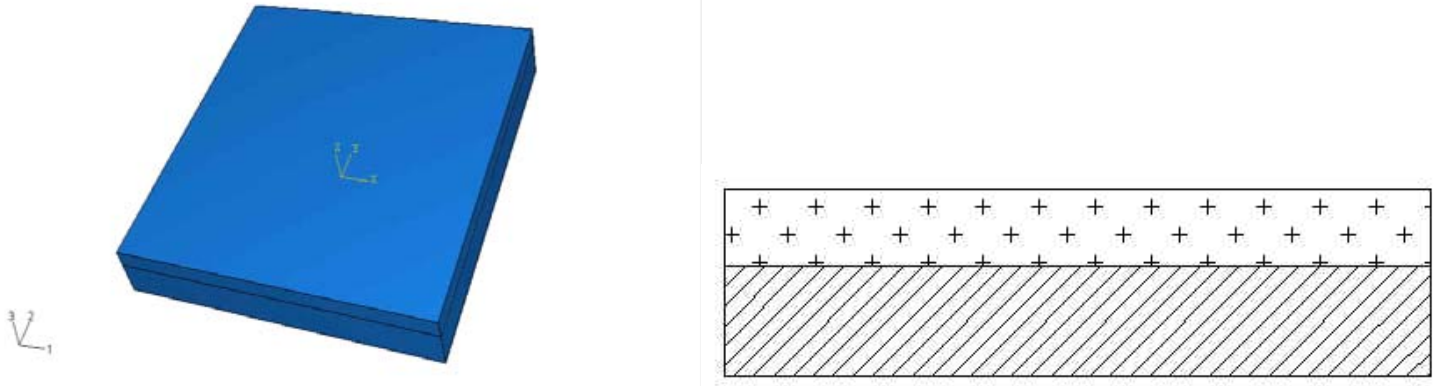

(2) Thermal growth of silicon dioxide on the silicon substrate. 

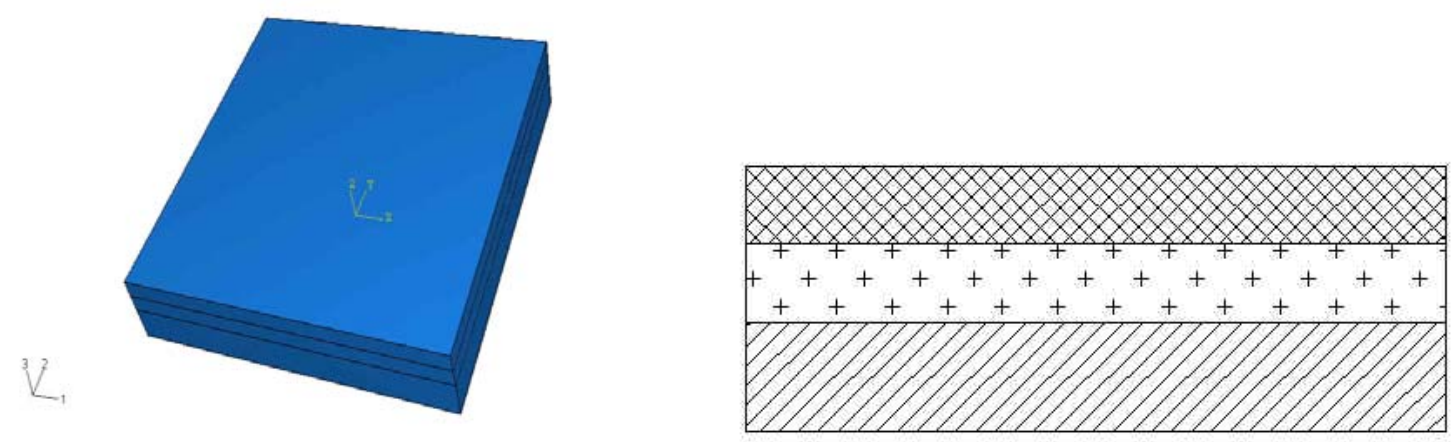

(3) Deposit first layer silicon on the top of silicon dioxide film.
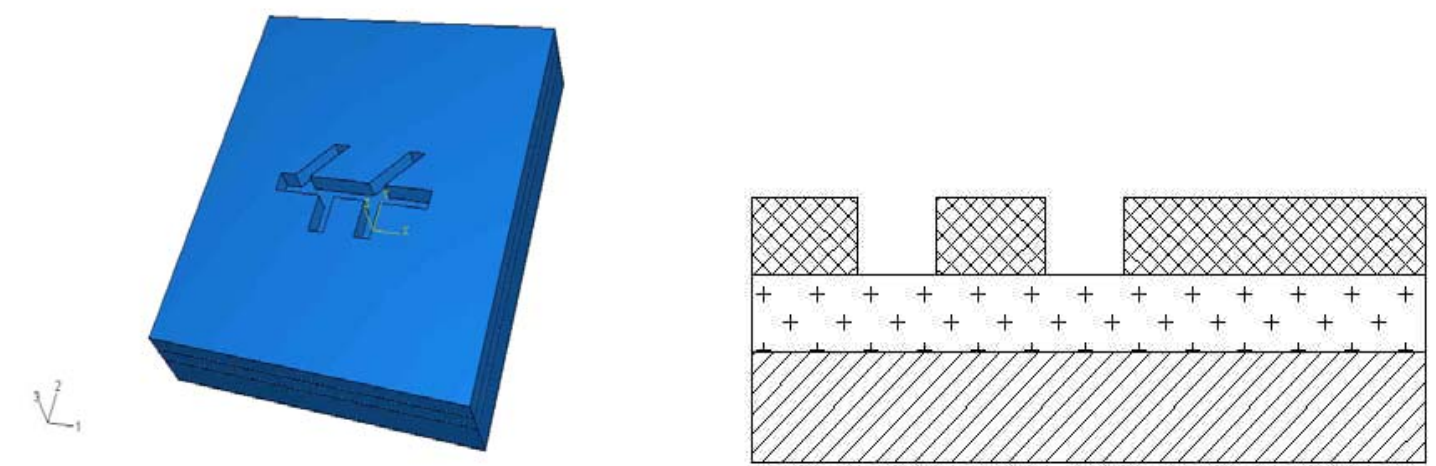

(4) Etch trenches in the first layer silicon to form interfaces with RIE.

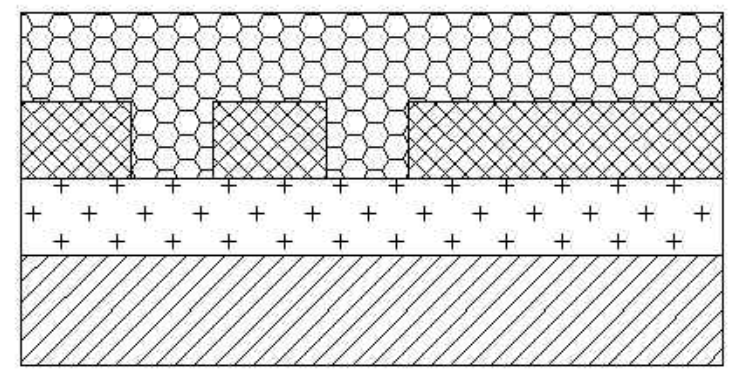

(5) Apply photoresist on the top of the etched first layer silicon to fill the trenches. 


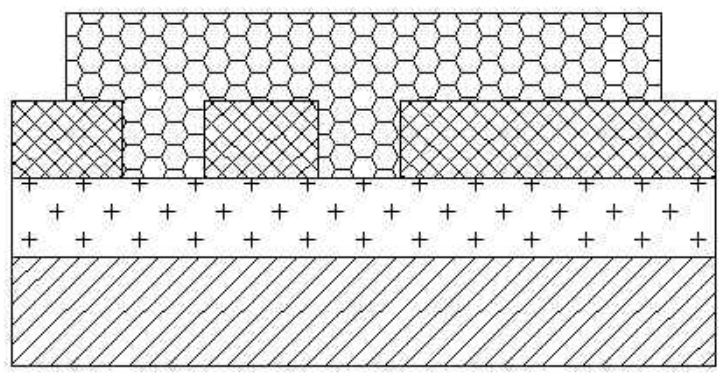

(6) Pattern the photoresist film; leave the photoresist only on the specimen area and remove the photoresist on the remaining areas using developer.

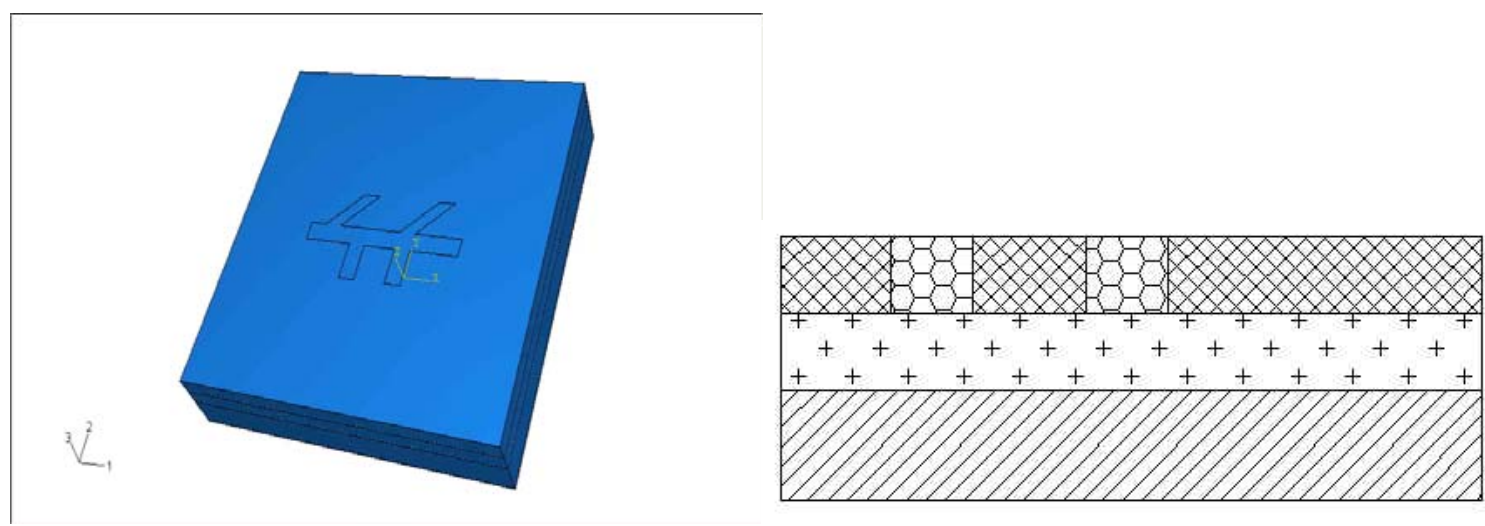

(7) Etch the photoresist on the top of the first layer silicon with RIE.
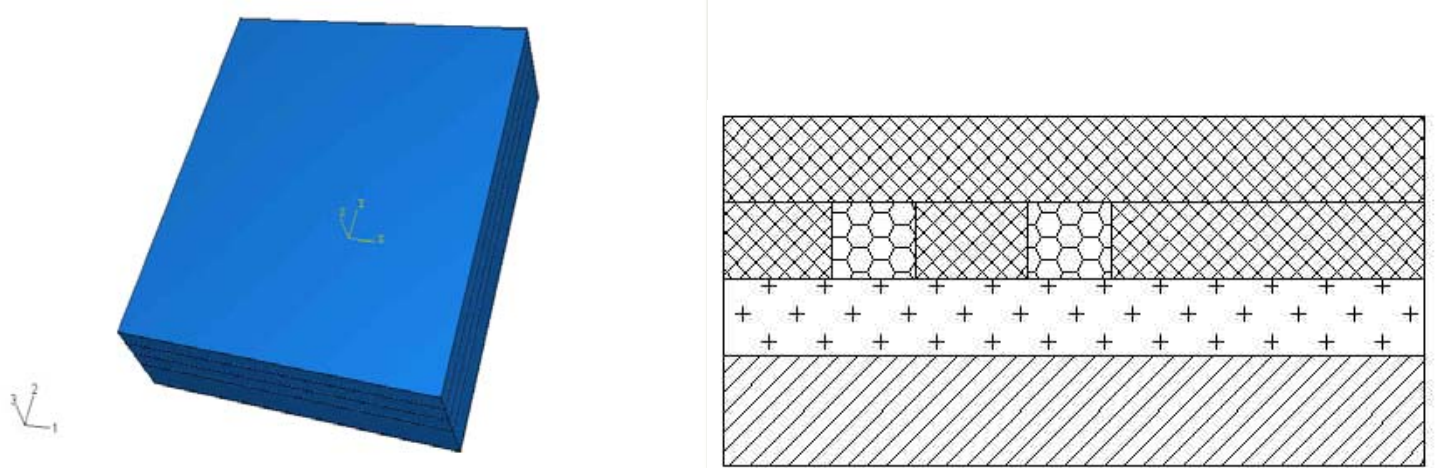

(8) Deposit Second layer silicon on the top of first layer silicon film. 

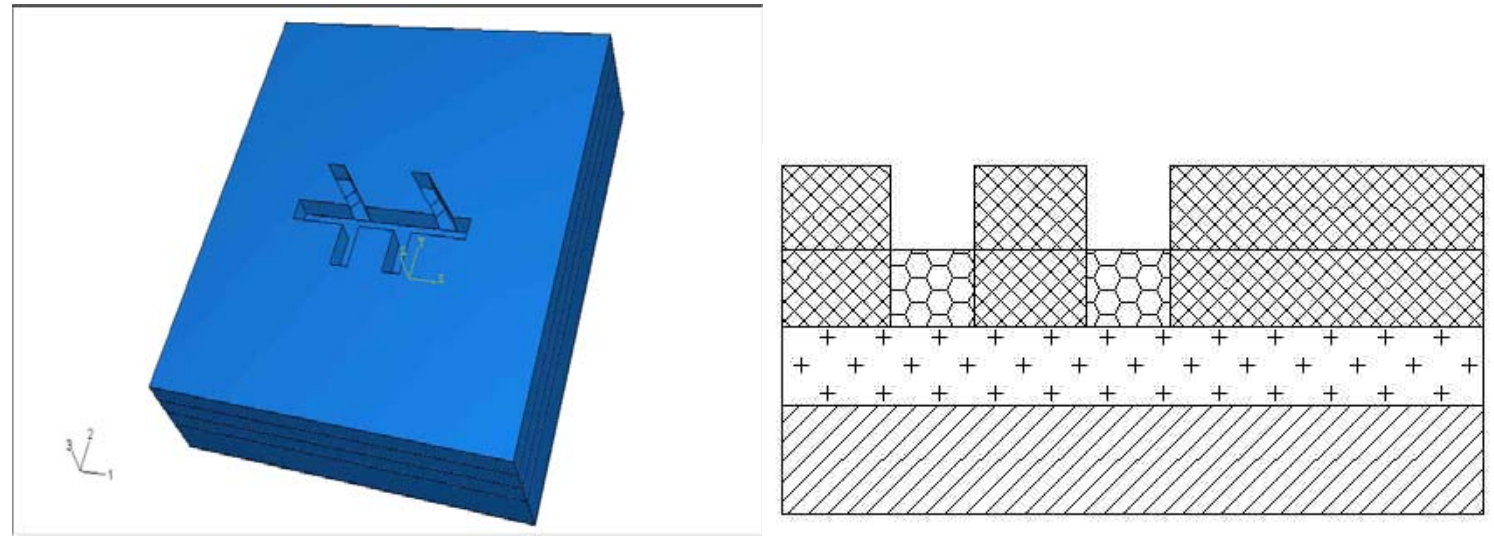

(9) Etch trenches in the second layer silicon to form interfaces with RIE.

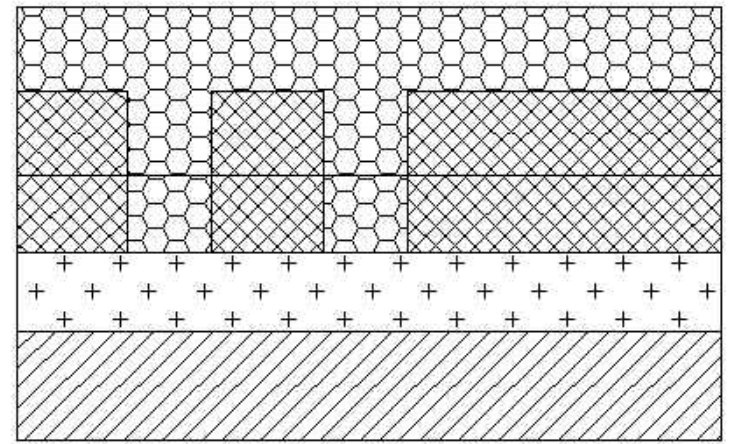

(10) Apply photoresist on the top of the etched second layer silicon to fill the trenches.

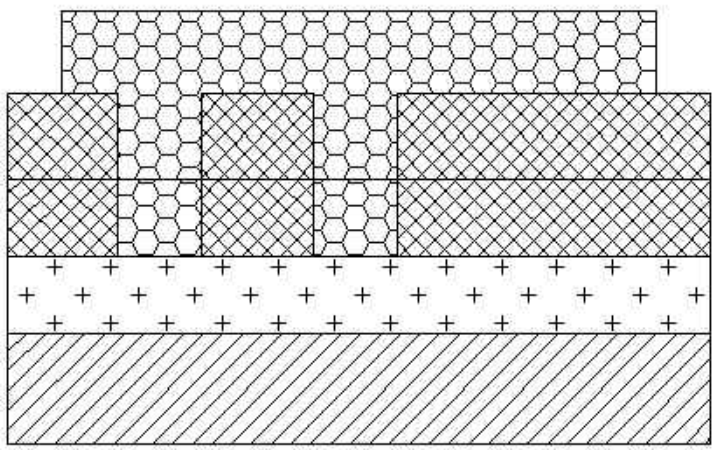

(11) Pattern the photoresist film; leave the photoresist only on the specimen area and remove the photoresist on the remaining areas using developer. 

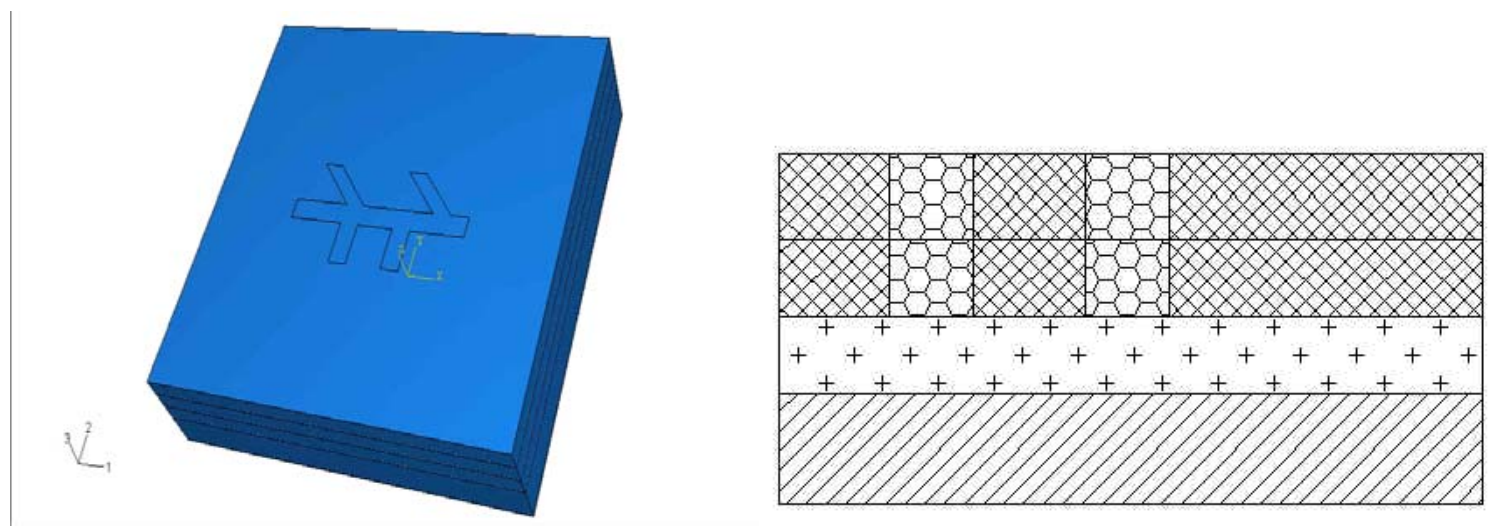

(12) Etch the photoresist on the top of the second layer silicon with RIE.
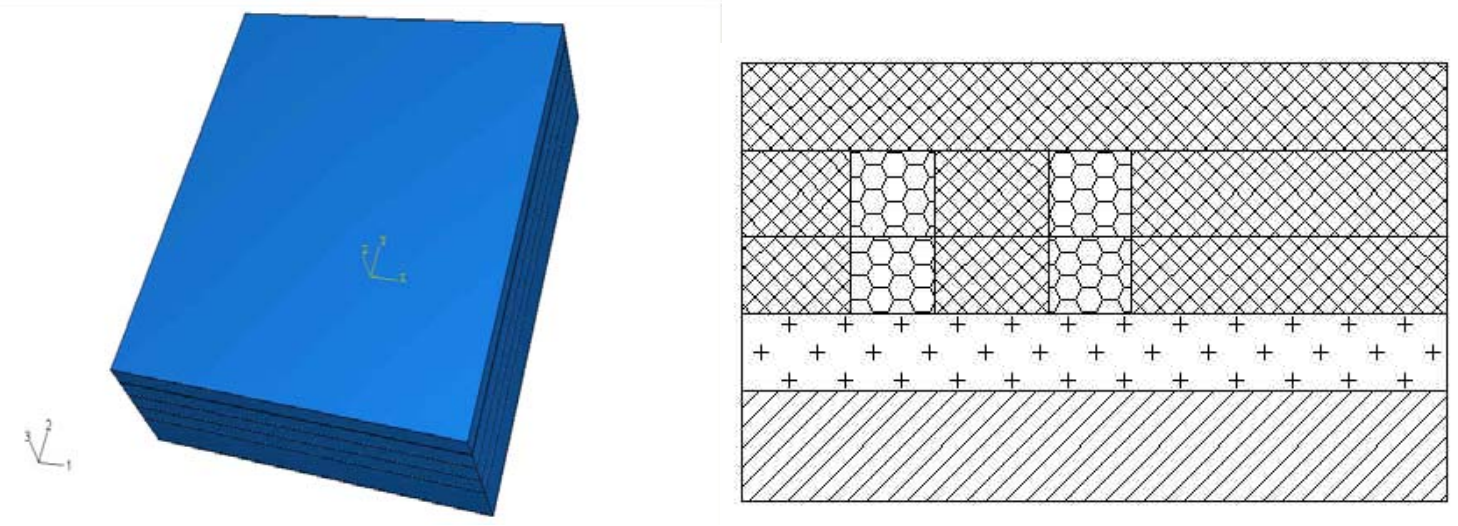

(13) Deposit Second layer silicon on the top of first layer silicon film.
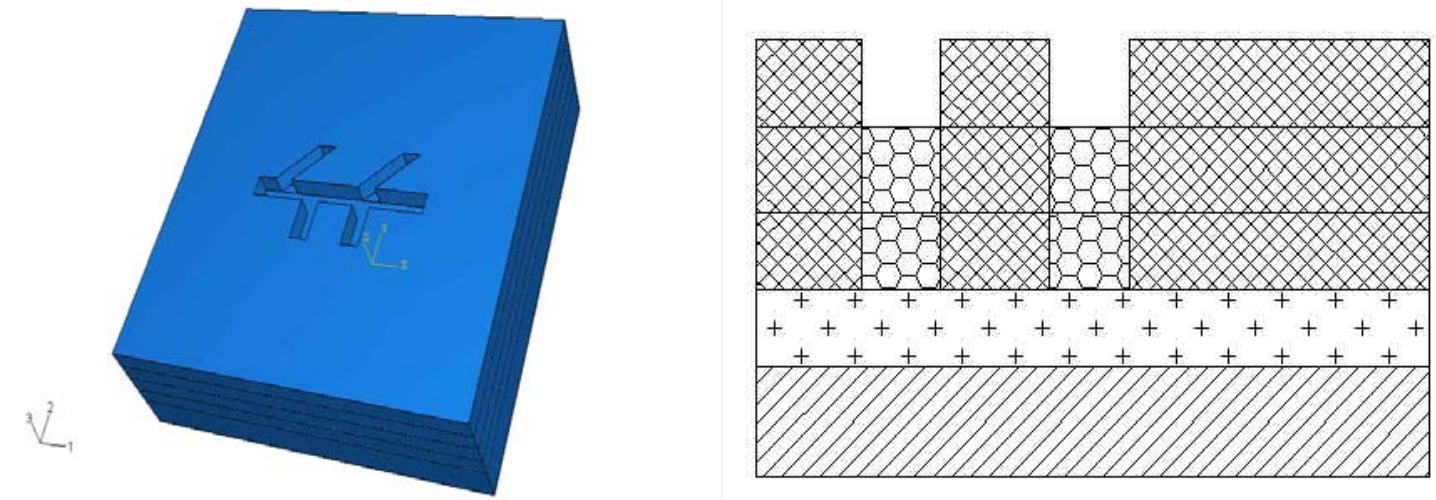

(14) Etch trenches in the third layer silicon to form interfaces with RIE. 


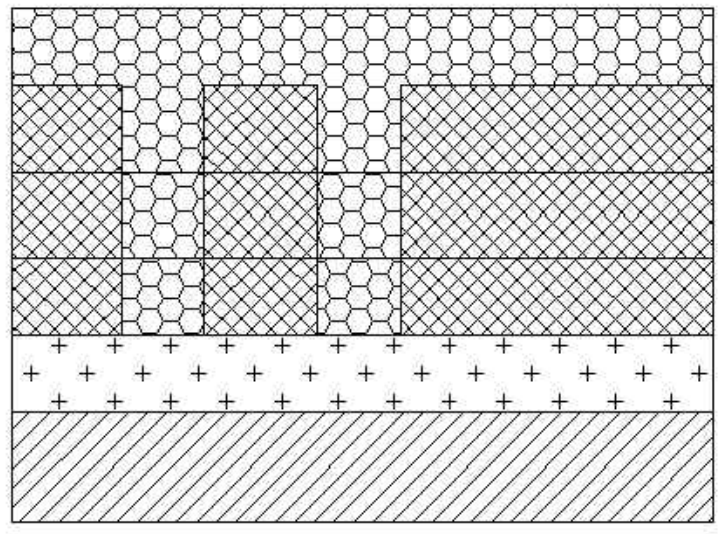

(15) Apply photoresist on the top of the etched second layer silicon to fill the trenches.

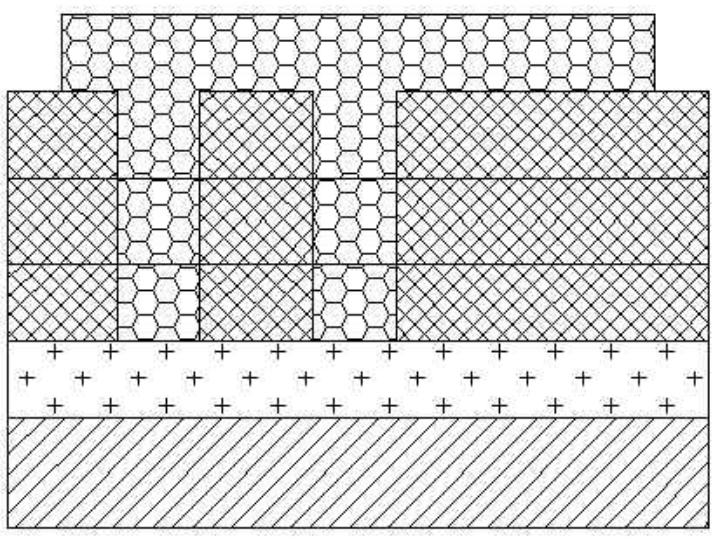

(16) Pattern the photoresist film; leave the photoresist only on the specimen area and remove the photoresist on the remaining areas using developer.
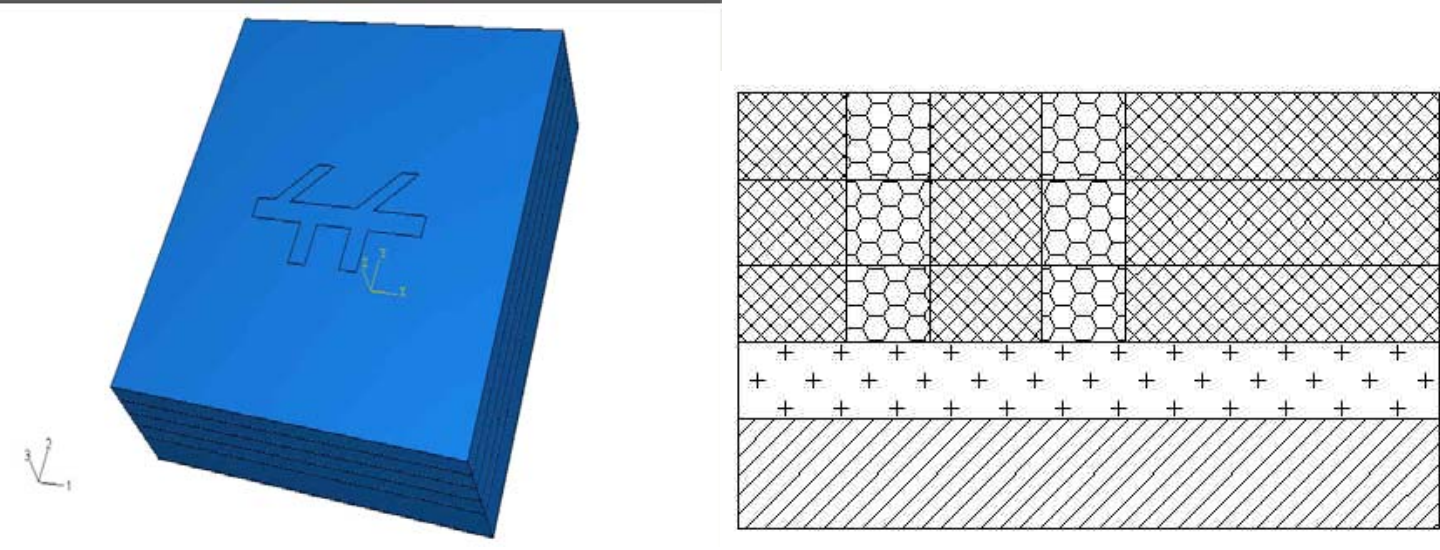

(17) Etch the photoresist on the top of the third layer silicon with RIE. 

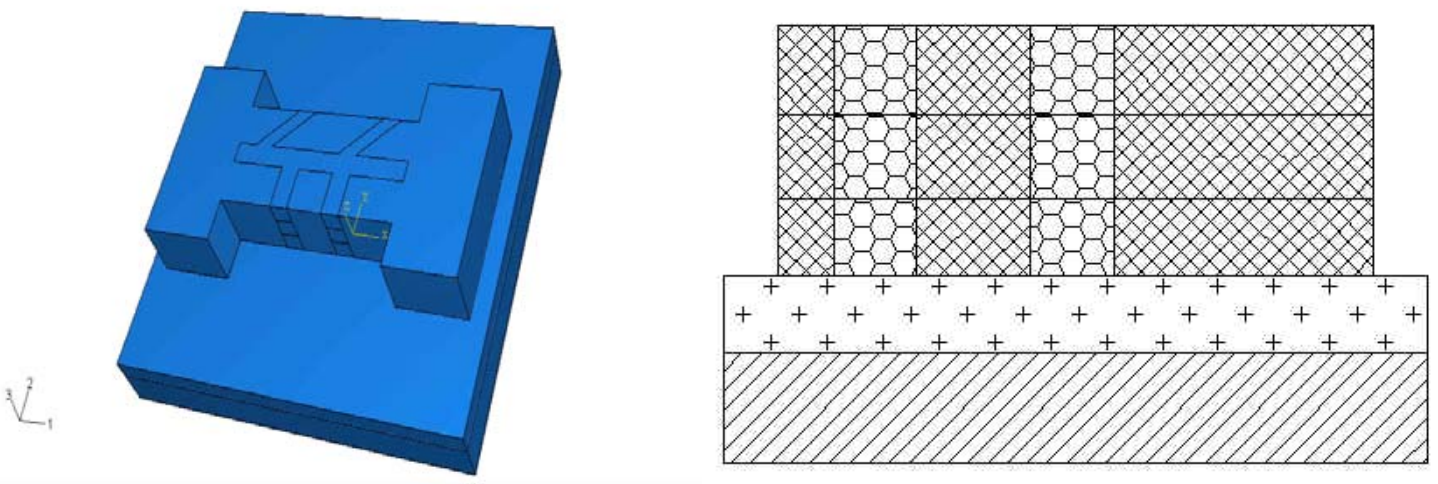

(18) Etch the three silicon layers down to the silicon dioxide layer to form the specimen shape.
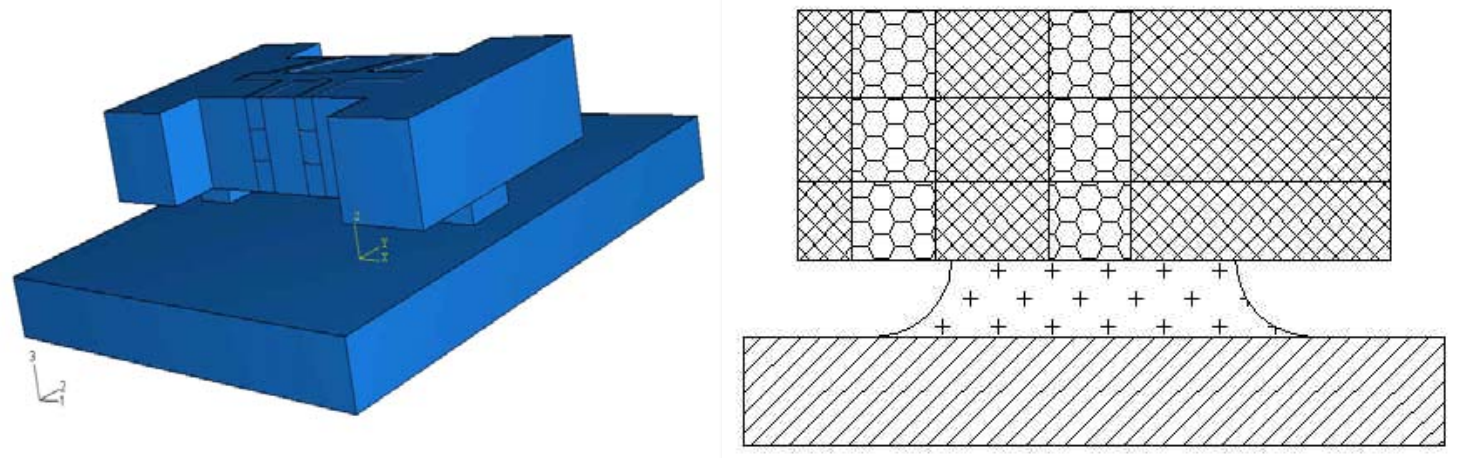

(19) Release the specimen using BOE to etch silicon dioxide film
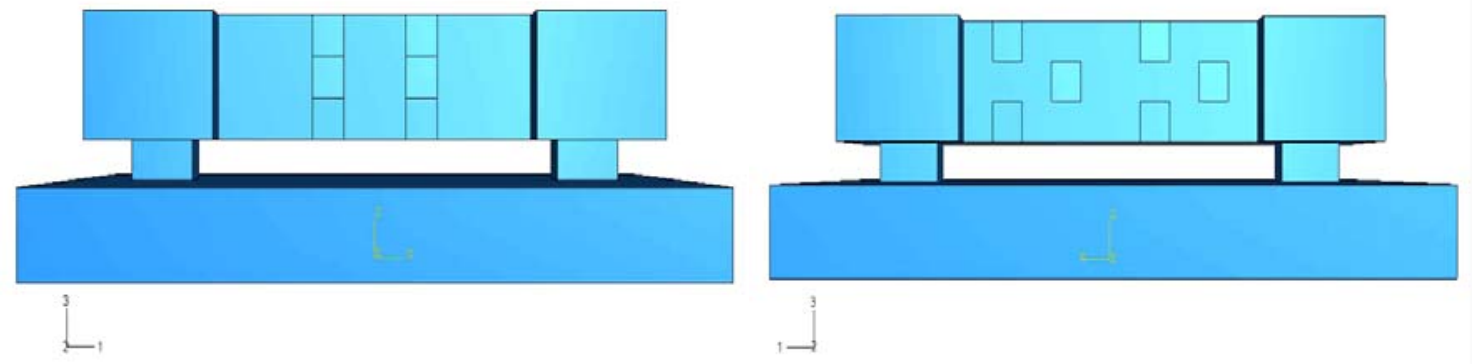

(20) 3-D profile views of the front and back side of released specimen.

Figure 2.4 (0)--(20) Sketches of the fabrication design. 


\subsubsection{Microstructure and photomask design}

Photomask is used to realize the microstructure design and transfer the structural features onto the silicon wafer. The photomask design is directly related to the microstructure geometry. A number of factors can influence the final designs of the photomask and the microstructure.

First, fabrication facilities have their limits, which prevent feature size smaller than two microns from being formed. Second, the nanoindenter, which is used for the mechanical property test of the microstructure, also has its limit in the load-control mode or in the displacement-control mode. Moreover, the size of the indenter tip and the machine stiffness require the sample beam to have a relatively long span. All of these factors restrict the microstructure geometry to a narrow scope.

In the Strombus gigas shell, the organic material layer is much thinner than the hard calcium carbonate layer. This means that the widths of the trenches formed in the silicon film should have the minimum feature size that can be transferred by the photolithography tools. This minimum feature size is about $2 \mu \mathrm{m}$.

The spacing between two trenches is another key dimension that needs to be determined. In the middle layer, the trenches separating the second-order lamellae should be dense enough to provide the crack stopping mechanism. Referred to the trench width of $2 \mu \mathrm{m}$, the spacing was chosen as $4 \mu \mathrm{m}$.

In order to generate tunneling cracks, the trenches in the inner layer should be dense and the width ratio of the inner layer and the middle layer should be small ${ }^{[7]}$. However, some problems restrict the application of the criterion. First, the depth of the microstructure should be smaller than $10 \mu \mathrm{m}$ since the etching tool in MFL cannot etch 
silicon film thicker than $10 \mu \mathrm{m}$. Therefore, the width of the microstructure should not be larger than $20 \mu \mathrm{m}$; otherwise, lateral buckling may happen and cause problems in determining the mechanical properties of the synthetic microstructure. On the other hand, the lengths of the trenches have to be much larger than the widths so that thin and long interface layers can be formed. Therefore, the lengths of the trenches have to be $8 \sim 10 \mu \mathrm{m}$. Thus, the widths of the inner layer and the middle layer cannot differ too much from each other.

Secondly, the volume fraction of the photoresist should be as small as possible according to the very small volume fraction of the organic material that existed in the shell structure. Since dense trenches have already been designed in the middle layer, the spacing between two trenches in the inner layer has to be relatively large to keep the volume fraction of the photoresist small.

The spacing between two trenches in the inner layer was chosen to be $18 \mu \mathrm{m}$ and the widths of the inner layer and the middle layer were set to be equal.

The length of the microstructure will be adjusted to satisfy the testing machine requirements. The microstructure will deflect in the direction parallel to the substrate plane. Therefore, the testing machine should be able to apply lateral load. Commercially available nanoindenters have this capability. They have submicron and sub-microNewton resolution for displacement control and load control, respectively. The stiffness of the loading cell system is about $8000 \mathrm{~N} / \mathrm{m}$ (MTS NanoindenterXP). The lateral test needs to use a customized nanoindenter tip, which has vertical sidewall and flat tip end. This particular tip can be made of a diamond cylinder with a cross-section diameter of 
$60 \sim 100 \mu \mathrm{m}$. Thus, the length of the microstructure should be larger than $100 \mu \mathrm{m}$ so that there is enough space to locate the tip.

Moreover, the length should be long enough to make the effective stiffness of the synthetic microstructure much smaller than $8000 \mathrm{~N} / \mathrm{m}$. In order to evaluate the effective stiffness of the microstructure, a finite element simulation was conducted with a trial beam length of $200 \mu \mathrm{m}$. Composite mechanics was employed to generate a simplified mechanics model for simulating the microstructure.

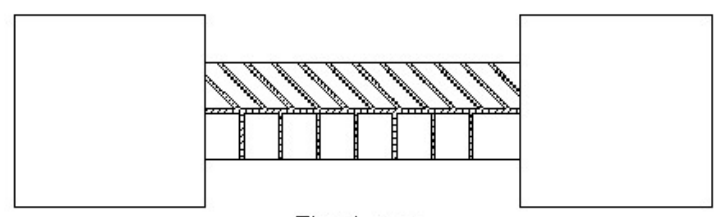

First Layer

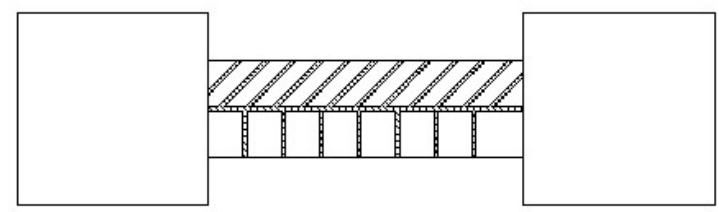

Second Layer

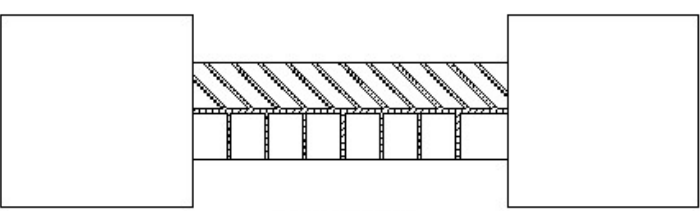

Third Layer

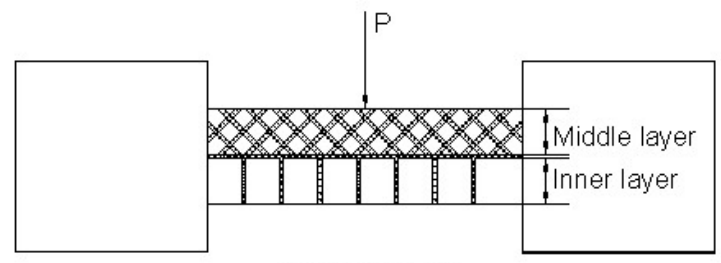

Final Structure

Figure 2.5 Illumination of the microstructure and the load direction.

Figure 2.5 sketches the basic configuration of the microstructure and the load direction. The microstructure is comprised of three layers stacked up in the depth direction. The trench directions in the middle layer are arranged in the way of $-45^{0} / 45^{0} /-$ $45^{\circ}$. As shown in Figure 2.6, a silicon plate with embedded photoresist bars will have a stiffness matrix with the following form ${ }^{[18][19]}$ :

$$
\left[\begin{array}{l}
\sigma_{1} \\
\sigma_{2} \\
\tau_{6}
\end{array}\right]=\left[\begin{array}{ccc}
Q_{11} & Q_{12} & 0 \\
Q_{12} & Q_{22} & 0 \\
0 & 0 & Q_{66}
\end{array}\right]\left[\begin{array}{l}
\varepsilon_{1} \\
\varepsilon_{2} \\
\gamma_{6}
\end{array}\right]
$$




$$
\begin{array}{ll}
Q_{11}=\frac{E_{1}}{1-v_{12} v_{21}} & Q_{12}=\frac{v_{12} E_{2}}{1-v_{12} v_{21}}=\frac{v_{21} E_{1}}{1-v_{12} v_{21}} \\
Q_{22}=\frac{E_{2}}{1-v_{12} v_{21}} & Q_{66}=G_{12}
\end{array}
$$

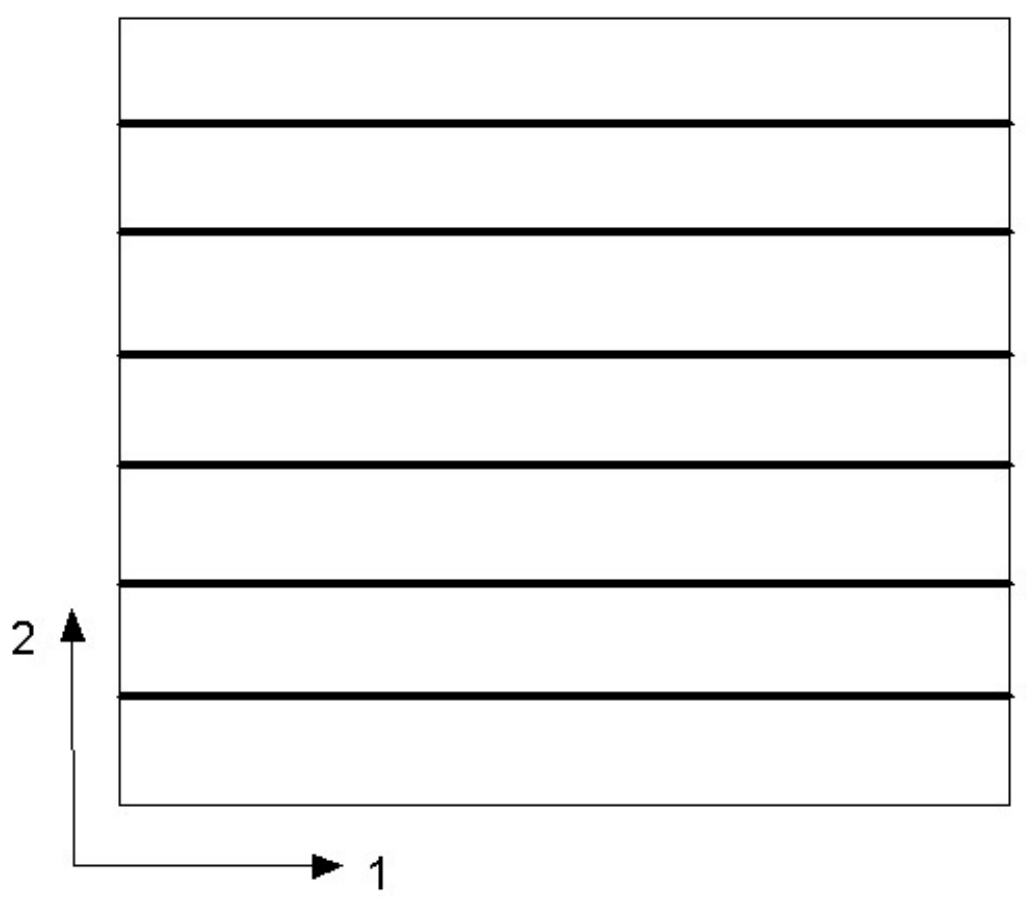

Figure 2.6 Laminate with silicon matrix and photoresist bars in 1-2 plane.

The principal moduli associated with the principal material direction 1-2 can be calculated based on the equally strained or equally stressed model. Along the 1-direction, the model assumes that the silicon and photoresist are equally strained and the Young's modulus $\mathrm{E}_{1}$ and Poisson's ratio $v_{12}$ are expressed as:

$$
\begin{aligned}
& E_{1}=V_{s i} E_{s i}+V_{p r} E_{p r} \\
& v_{12}=V_{s i} v_{s i}+V_{p r} v_{p r}
\end{aligned}
$$

Here, $\mathrm{V}_{\mathrm{si}}$ and $\mathrm{V}_{\mathrm{pr}}$ are volume fraction of silicon and photoresist in the lamina, respectively. 
Along the 2-direction, the model assumes that the silicon and photoresist are equally stressed:

$$
\begin{aligned}
& \frac{1}{E_{2}}=\frac{V_{s i}}{E_{s i}}+\frac{V_{p r}}{E_{p r}} \\
& \frac{1}{G_{12}}=\frac{V_{s i}}{G_{s i}}+\frac{V_{p r}}{G_{p r}} \\
& G=\frac{E}{2(1+v)} \\
& \frac{v_{12}}{E_{1}}=\frac{v_{21}}{E_{2}}
\end{aligned}
$$

Here, $\mathrm{E}_{\mathrm{si}}$ and $v_{\mathrm{si}}$ were chosen as $160 \mathrm{GPa}$ and $0.22 ; \mathrm{E}_{\mathrm{pr}}$ and $v_{\mathrm{pr}}$ were chosen as $1 \mathrm{GPa}$ and 0.3 . For a $200 \mu \mathrm{m}$ long beam, $\mathrm{V}_{\mathrm{si}}=0.69, \mathrm{~V}_{\mathrm{pr}}=0.31$. The following parameters were calculated as:

$$
\begin{aligned}
& \mathrm{E}_{1}=110.71 \mathrm{GPa} \\
& v_{12}=0.24 \\
& \mathrm{G}_{\mathrm{pr}}=0.38 \mathrm{GPa}, \mathrm{G}_{\mathrm{si}}=65.57 \mathrm{GPa} \\
& \mathrm{E}_{2}=3.18 \mathrm{GPa} \\
& \mathrm{G}_{12}=1.21 \mathrm{GPa} \\
& v_{21}=0.007
\end{aligned}
$$

Thereafter, the stiffness matrix in the 1-2 plane was obtained as follows:

$$
\left[\begin{array}{ccc}
Q_{11} & Q_{12} & 0 \\
Q_{12} & Q_{22} & 0 \\
0 & 0 & Q_{66}
\end{array}\right]=\left[\begin{array}{ccc}
110.9 & 0.76 & 0 \\
0.76 & 3.19 & 0 \\
0 & 0 & 1.21
\end{array}\right]
$$


After rotating the direction of the photoresist by an angle $\theta$ as shown in Figure 2.7, the new structure will have an effective stiffness matrix in X-Y plan as follows:

$$
\begin{aligned}
& {\left[\begin{array}{lll}
Q_{X X} & Q_{X Y} & 2 Q_{X S} \\
Q_{Y X} & Q_{Y Y} & 2 Q_{Y S} \\
Q_{S X} & Q_{S Y} & 2 Q_{S S}
\end{array}\right]=T^{-1}\left[\begin{array}{ccc}
Q_{11} & Q_{12} & 0 \\
Q_{12} & Q_{22} & 0 \\
0 & 0 & 2 Q_{66}
\end{array}\right] T} \\
& T=\left[\begin{array}{ccc}
m^{2} & n^{2} & 2 m n \\
n^{2} & m^{2} & -2 m n \\
-m n & m n & m^{2}-n^{2}
\end{array}\right] \quad m=\cos \theta \quad n=\sin \theta
\end{aligned}
$$

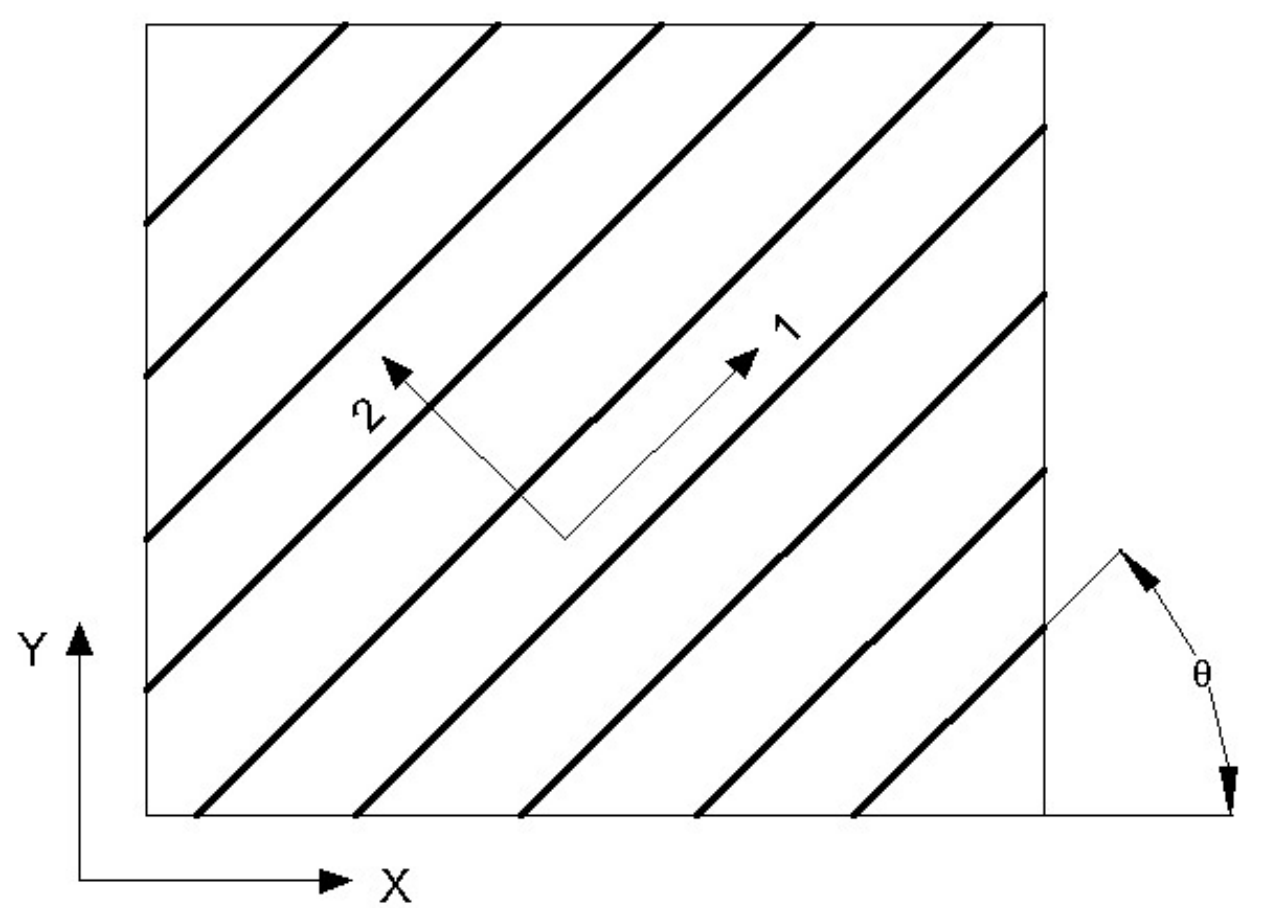

Figure 2.7 Principal material directions rotate an angle $\theta$ with respect $X$-axis in $X-Y$ plane. 
For the first, second and third layer in the middle layer, $\theta$ was equal to $-45^{0} / 45^{0} /-$ $45^{\circ}$, respectively. The corresponding stiffness matrixes in X-Y plane were calculated:

$$
\begin{aligned}
& Q^{1}=\left[\begin{array}{lll}
Q_{X X} & Q_{X Y} & Q_{X S} \\
Q_{Y X} & Q_{Y Y} & Q_{Y S} \\
Q_{S X} & Q_{S Y} & Q_{S S}
\end{array}\right]=\left[\begin{array}{ccc}
30.11 & 27.69 & -26.93 \\
27.69 & 30.11 & -26.93 \\
-26.93 & -26.93 & 28.14
\end{array}\right] \\
& Q^{2}=\left[\begin{array}{lll}
Q_{X X} & Q_{X Y} & Q_{X S} \\
Q_{Y X} & Q_{Y Y} & Q_{Y S} \\
Q_{S X} & Q_{S Y} & Q_{S S}
\end{array}\right]=\left[\begin{array}{lll}
30.11 & 27.69 & 26.93 \\
27.69 & 30.11 & 26.93 \\
26.93 & 26.93 & 28.14
\end{array}\right] \\
& Q^{3}=\left[\begin{array}{lll}
Q_{X X} & Q_{X Y} & Q_{X S} \\
Q_{Y X} & Q_{Y Y} & Q_{Y S} \\
Q_{S X} & Q_{S Y} & Q_{S S}
\end{array}\right]=\left[\begin{array}{ccc}
30.11 & 27.69 & -26.93 \\
27.69 & 30.11 & -26.93 \\
-26.93 & -26.93 & 28.14
\end{array}\right]
\end{aligned}
$$

The global effective stiffness matrix of the middle layer in X-Y plane can be expressed as:

$$
\left[\begin{array}{l}
N \\
M
\end{array}\right]=\left[\begin{array}{ll}
A & B \\
B & D
\end{array}\right]\left[\begin{array}{l}
\varepsilon \\
\kappa
\end{array}\right]
$$

This so-called ABD matrix is expressed as:

$$
\begin{aligned}
& A_{i j}=\sum_{k=1}^{n} Q_{i j}^{k}\left(z_{k}-z_{k-1}\right) \\
& B_{i j}=\frac{1}{2} \sum_{k=1}^{n} Q_{i j}^{k}\left(z_{k}^{2}-z_{k-1}^{2}\right) \\
& A_{i j}=\frac{1}{3} \sum_{k=1}^{n} Q_{i j}^{k}\left(z_{k}^{3}-z_{k-1}^{3}\right)
\end{aligned}
$$

The middle layer can be considered as an angle-ply composite and the global engineering Young's modulus and Poisson's ratio are related to matrix A with the following relationship: 


$$
\begin{array}{ll}
\bar{E}_{X}=\frac{A_{X X} A_{Y Y}-A_{X Y}^{2}}{H A_{Y Y}} & \bar{v}_{X Y}=\frac{A_{X Y}}{A_{Y Y}} \\
\bar{E}_{Y}=\frac{A_{X X} A_{Y Y}-A_{X Y}^{2}}{H A_{X X}} & \bar{v}_{Y X}=\frac{A_{X Y}}{A_{X X}}
\end{array}
$$

Here, $\mathrm{H}$ is the total depth of the middle layer. Assumed that the depth of each layer was $3 \mu \mathrm{m}$, and then $\mathrm{H}$ was $9 \mu \mathrm{m}$. Thus, matrix A is expressed as:

$$
A=3 Q^{1}+3 Q^{2}+3 Q^{3}=\left[\begin{array}{rrr}
271.01 & 249.23 & -80.78 \\
249.23 & 271.01 & -80.78 \\
-80.78 & -80.78 & 253.28
\end{array}\right]
$$

So $\bar{E}_{X}, \bar{v}_{X Y}, \bar{E}_{Y}$ and $\bar{v}_{Y X}$ were calculated as:

$$
\bar{E}_{X}=\bar{E}_{Y}=4.65 G P a \quad \bar{v}_{X Y}=\bar{v}_{Y X}=\frac{A_{X Y}}{A_{Y Y}}=0.92
$$

The calculated Poisson's ratio was greater than 0.5 , which was impossible for engineering materials. Therefore, the effective Poisson's ratio of the middle layer was set to be 0.3 .

The middle layer is an isotropic material where the effective Young's modulus and Poisson's ratio are equal to $4.65 \mathrm{GPa}$ and 0.3 , respectively. The inner layer includes two parts: photoresist and silicon. Thus, the microstructure is now comprised of three parts as shown in Figure 2.8. 

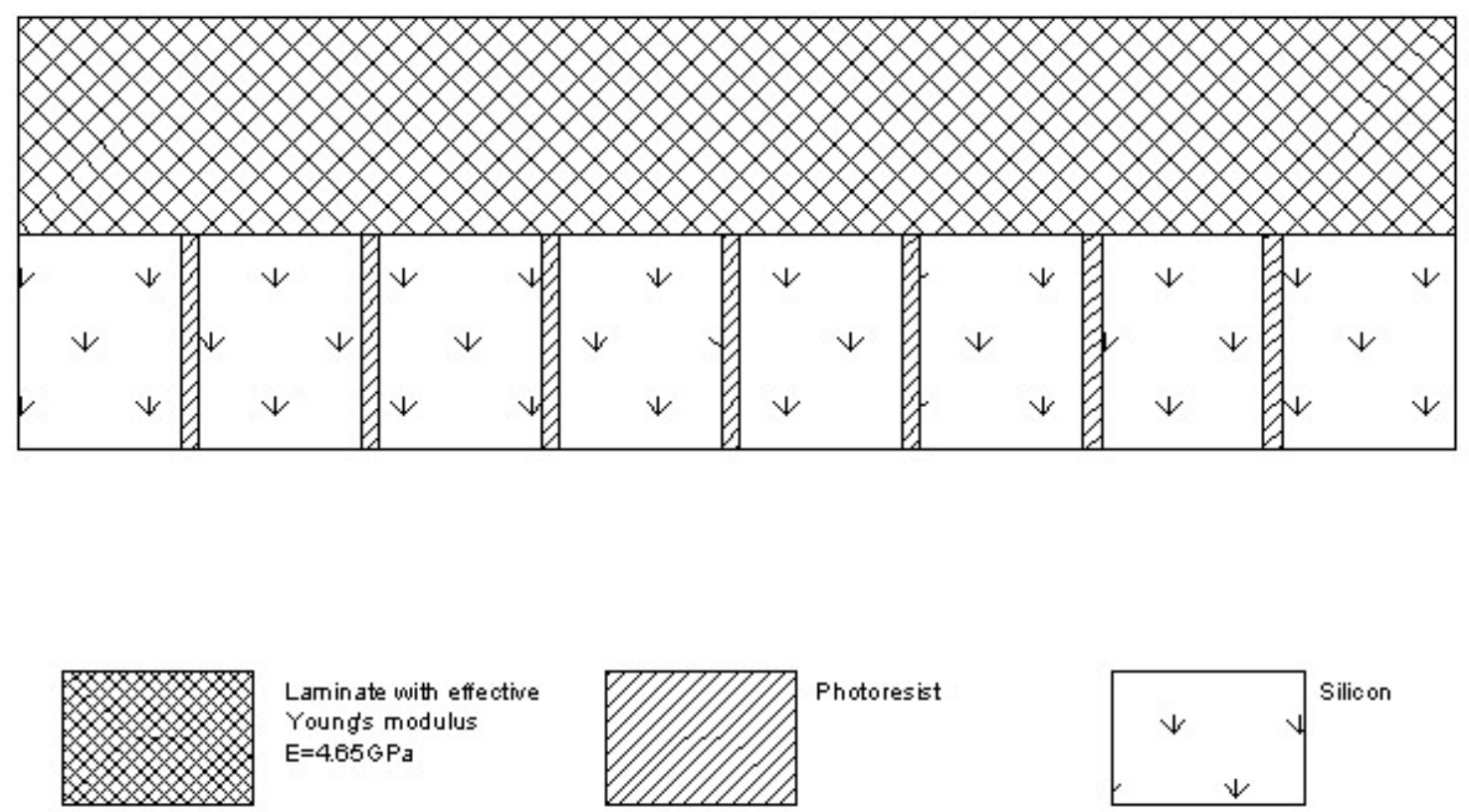

Lamin ate with effective

Young's modulus
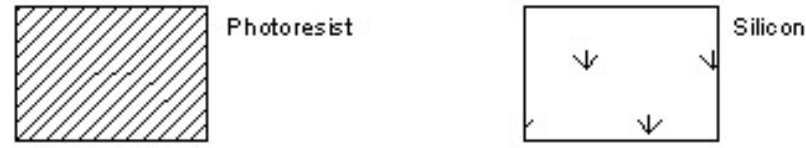

Figure 2.8 Sketch of the simplified microstructure.

The simplified model was implemented using ABAQUS with a 2-D configuration to represent the central plane of the microstructure according in the microstructure depth direction. Perfect bonding was used for the interfaces between any two materials and the microstructure was fixed at both ends. Plane stress quadratic element with quad shape was chosen to mesh the structure. For a $200 \mu \mathrm{m}$ long and $20 \mu \mathrm{m}$ wide beam, the simplified microstructure and its meshes are shown in Figure 2.9.

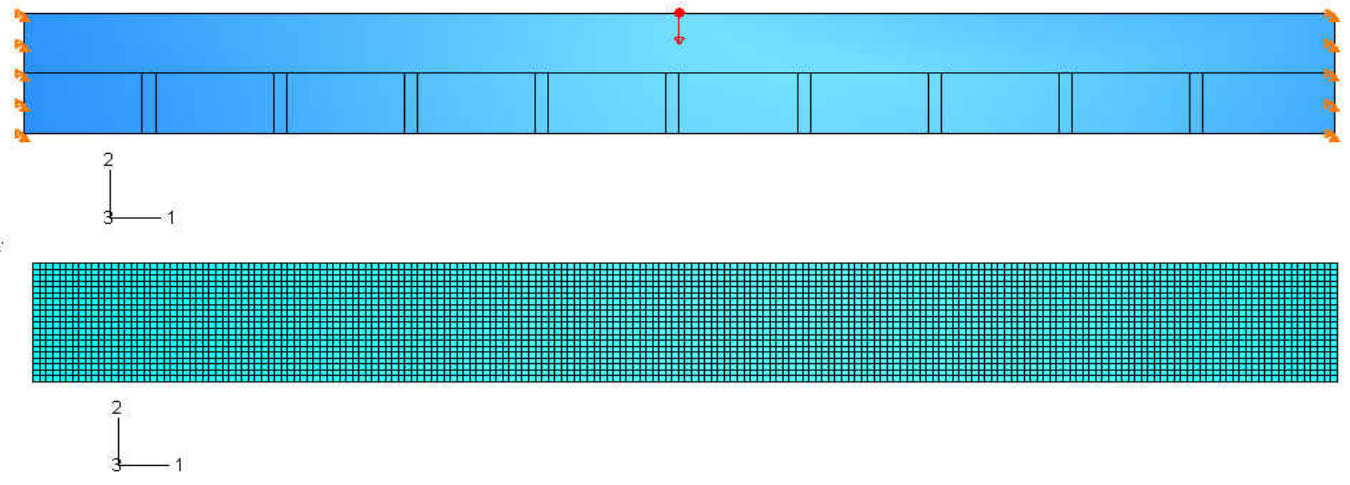

Figure 2.9 A meshed microstructure. 

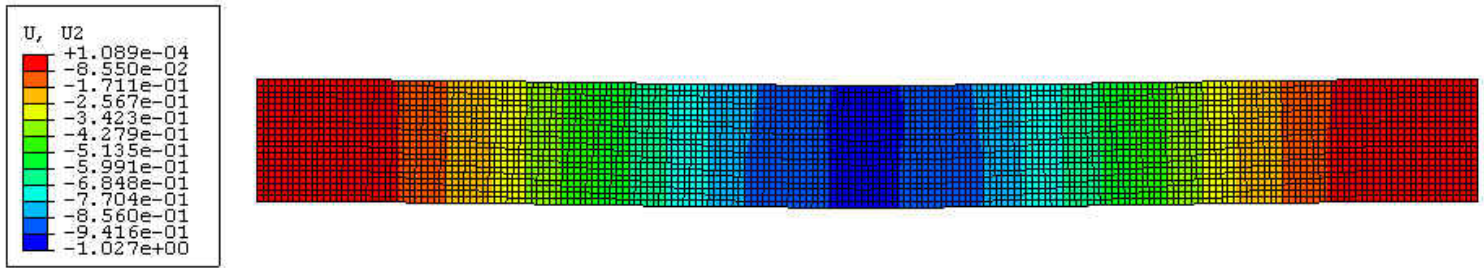

(a)

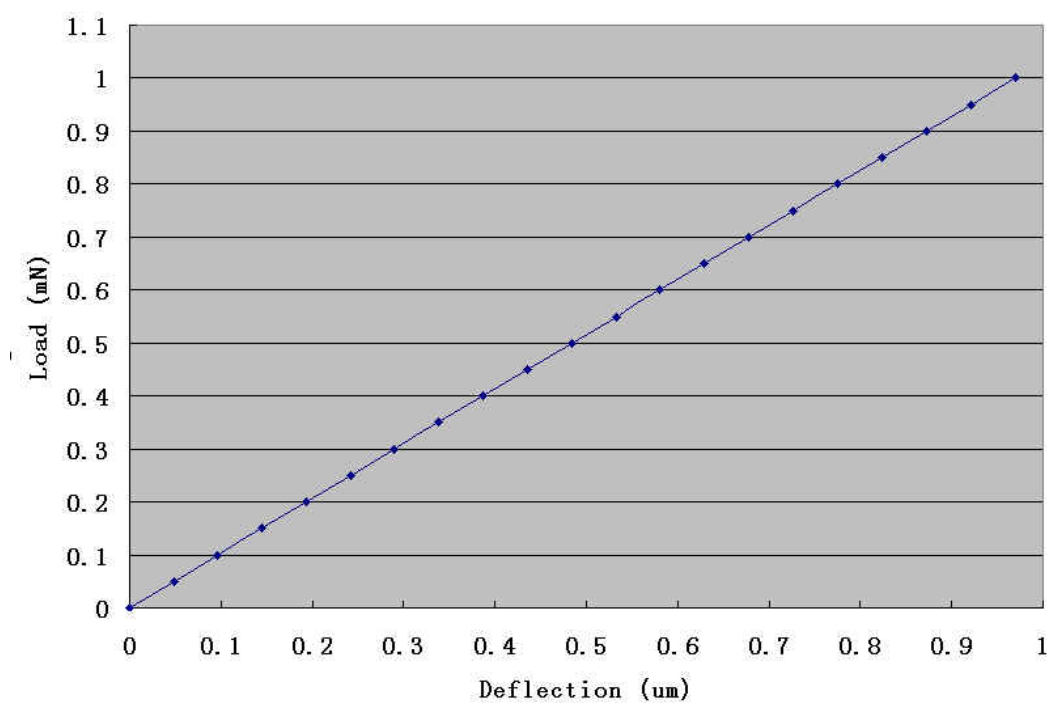

(b)

Figure 2.10 (a) Beam deformation (b) Load-deflection curve for the loading point.

Figure 2.10 shows the deformed microstructure under the load of $1 \mathrm{mN}$ and the load-deflection curve of the loading point. The effective flexural stiffness was obtained from the load-deflection curve with a value of $1032 \mathrm{~N} / \mathrm{m}$. This value is relatively small with respect to lateral stiffness of the nanoindenter, which is $8000 \mathrm{~N} / \mathrm{m}$. Therefore, the designed microstructure satisfies the requirements of the nanoindenter.

Based on the calculation above and the feasibility analysis of the fabrication, four configurations have been chosen and the dimensions in length and width are: $200 \cdot 20$, $200 \bullet 10,300 \cdot 20$, and $300 \bullet 10(\mu \mathrm{m} \bullet \mu \mathrm{m})$. 
The photomask design just follows the structure design and adds some features for the fabrication demands. A sketch of the die layout is shown in Figure 2.11. In each die, the designed microstructures with four distinct dimensions are located in different rows and columns. Each row of the microstructures is not simply duplicated from the upper or lower row. The microstructures with the same dimension are arrayed in different columns so that identical microstructures can be equably distributed in the die area. This arrangement reduces the influence of fabrication defects on the quality of the microstructures the furthest.

According to the fabrication flow, four photomasks are needed. The first mask is named as 'odd layer' and is a dark-field mask, which means that the feature area is clear glass and the rest of the area is covered by chrome. The sketch of this mask is shown in Figure 2.12. The grey area represents chrome and the clear area is the structural feature. Since the trench width is only $2 \mu \mathrm{m}$ and it is impossible to measure its etching depth using Dek-Tak in MFL, additional trenches with larger width have to be introduced in the silicon film. As shown in Figure 2.12, $20 \mu \mathrm{m}$ wide trenches are designed in the photomask for the measurement convenience. 


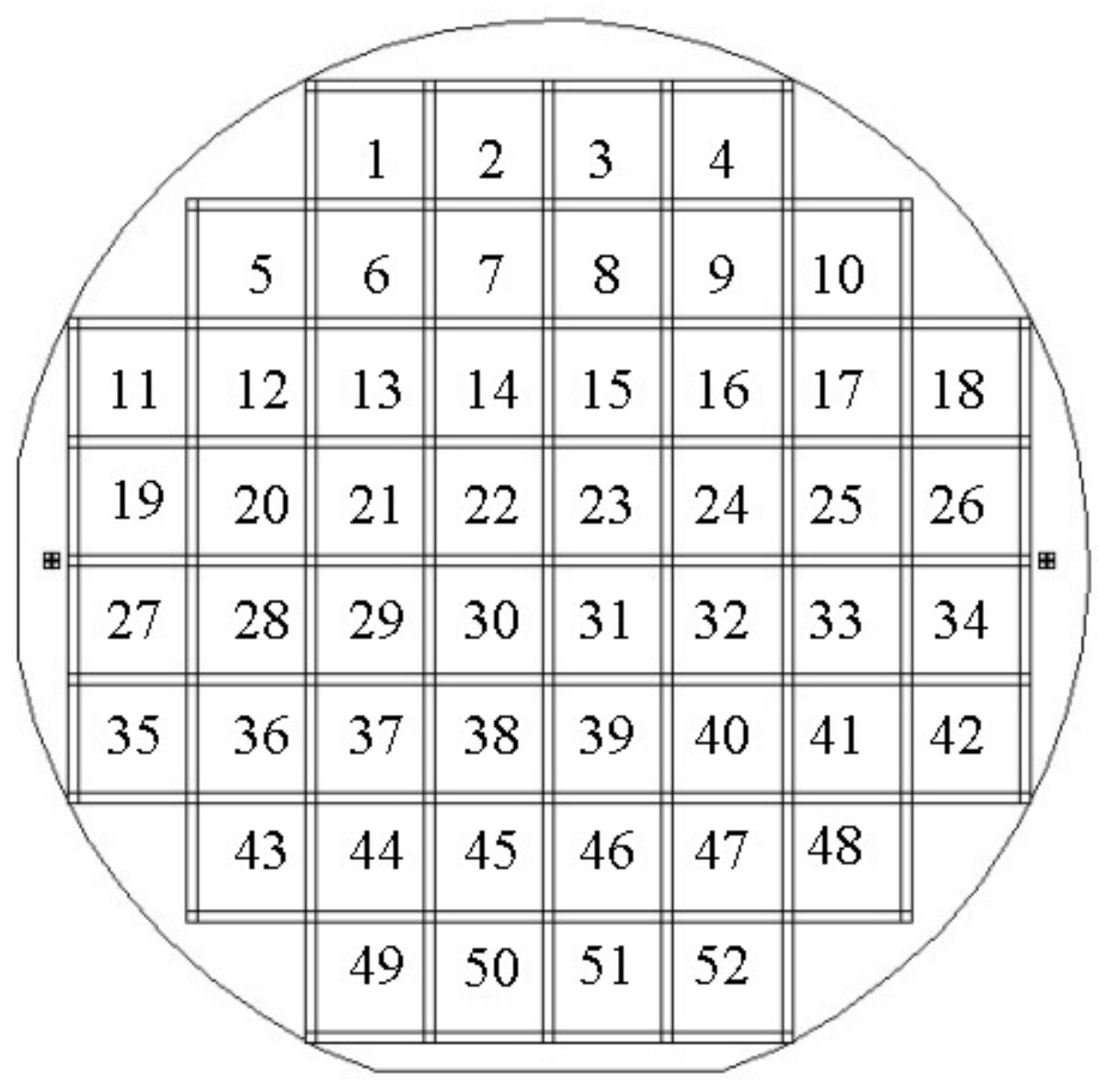

Figure 2.11 Sketch of the die layout.

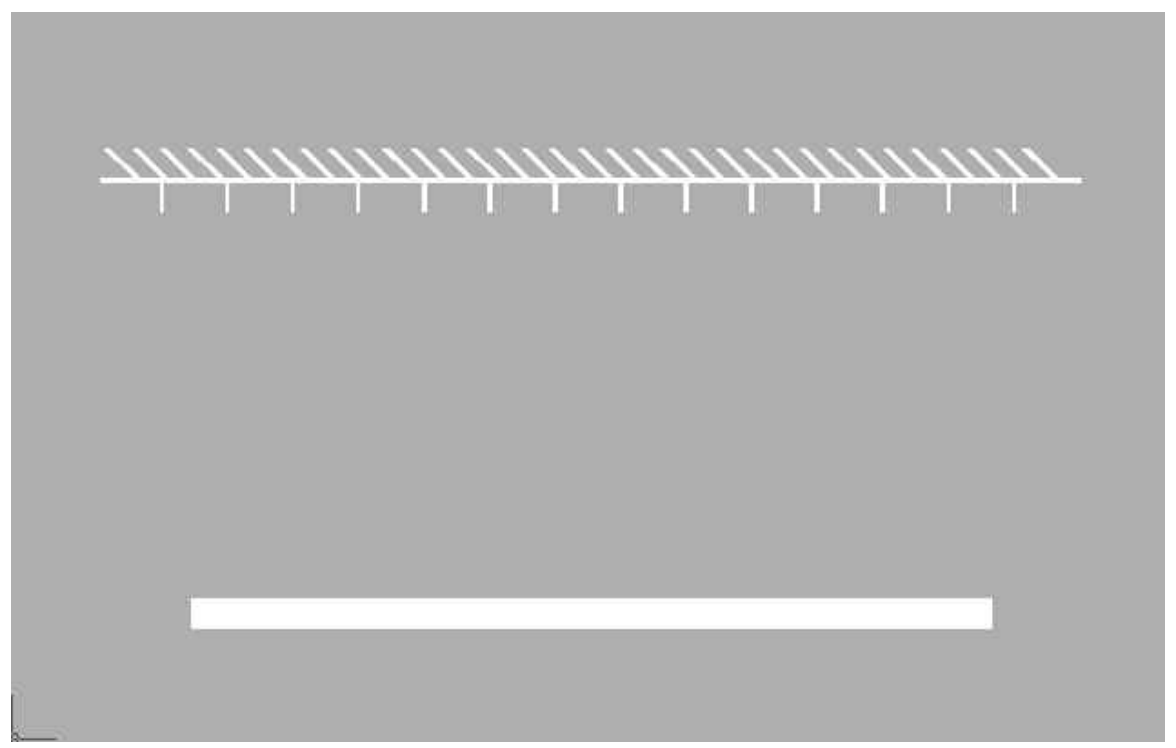

Figure 2.12 Sketch of the 'odd layer' mask 
The second mask is named as 'interface'. The function of this mask is to retain the photoresist on the top of the microstructure regions and remove the photoresist in the remaining area after filling photoresist in the trenches. This photomask is a clear-field mask, which means that the feature area is covered by chrome and the rest area is clear glass.

The third mask is named as 'even layer'. This mask is a dark-field mask and the feature arrangement is almost the same as the 'odd layer' mask except the directions of the inclined trenches are perpendicular to those in the 'odd layer' mask. Figure 2.13 is the top view of the two overlapped masks, mask 'odd layer' and mask 'even layer'.

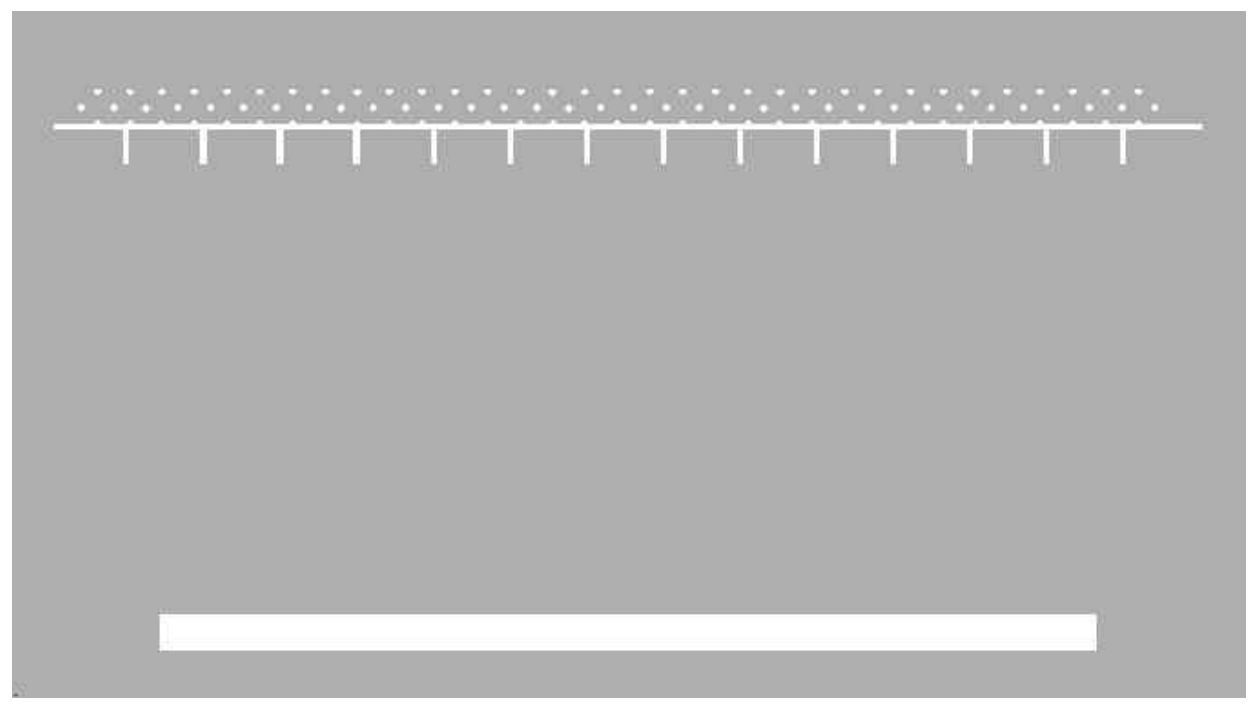

Figure 2.13 Sketch of the two overlapped masks, mask 'odd layer' and mask 'even layer'.

The fourth mask, which is a clear-field mask, is referred to the 'final structure'. In this mask, anchor geometry is assigned based on the microstructure width. During the structure release process, the releasing solution will etch the sacrificial layer isotropically. In order to completely release the microstructures and retain enough large anchors to 
provide the double-fixed boundary condition, the anchor dimension should be at least two times as large as the microstructure width.

In this design, the size of the anchor is $60 \mu \mathrm{m} \bullet 60 \mu \mathrm{m}$ for the $20 \mu \mathrm{m}$ wide microstructure, and $50 \mu \mathrm{m} \cdot 50 \mu \mathrm{m}$ for the $10 \mu \mathrm{m}$ wide microstructure. The sketch of the 'final structure' mask is shown in Figure 2.14. The grey area is covered by chrome and the rest area in the rectangular is clear glass.

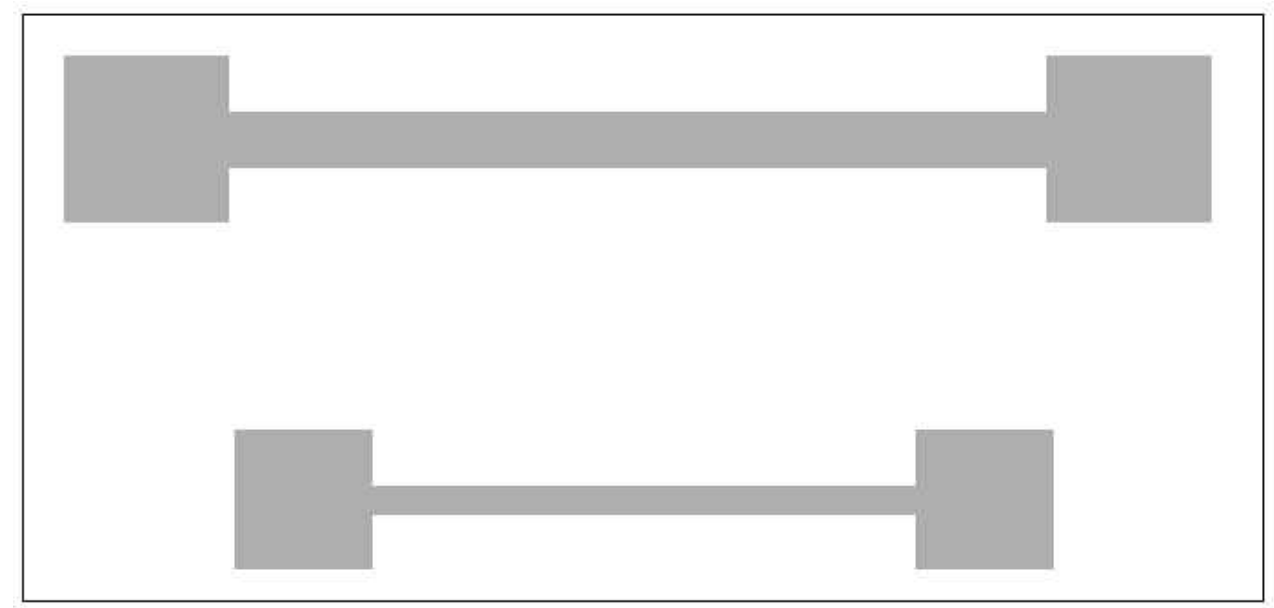

Figure 2.14 Sketch of the 'final structure' mask.

This photomask design was realized with AutoCAD and the masks were manufactured by DuPont, Inc. 


\section{Chapter Three}

\section{Fabrication}

Fabrication procedures will be introduced in this chapter. A detailed process flow is presented in Appendix A.1. Since this is the first attempt to fabricate a microcomposite structure, most of the processes in this thesis are non-standard. Processing problems might be encountered in almost every single fabrication step. Two processes are critical for the microstructure quality. One is trench etching in the silicon layers and another one is photoresist filling in the trenches. Most of the discussion is related to these two problems.

\subsection{First layer fabrication}

As described previously, once photoresist is filled in the trenches in the first layer, any processes that require operating temperature higher than $150{ }^{0} \mathrm{C}$ are not allowed. Thus, the traditional LPCVD method is not suitable for the deposition of the second and the third layers. There are two options for solving this problem. One is to use LPCVD to deposit polysilicon for the first layer and use the sputtering method to sputter silicon film for the second and the third layers.

The other option is to use the sputtering method for all of the three layers. However, there is an uncertain issue with this method; the bonding between the sacrificial $\mathrm{SiO}_{2}$ layer and the sputtered silicon film. The bonding between the thermally grown polysilicon film and the oxide film is tight and the possibility for the releasing solution to 
penetrate through the interface is very low. Low temperature processes definitely have higher probability to produce poor quality interface that might include gaps or defects. This will lower the adhesion between the silicon film and the oxide film, making infiltration through the interface possible for the releasing solution and causing the whole sacrificial layer to be etched away. Therefore, the first option is better than the second one and is chosen for the microstructure fabrication.

The wafers used for the fabrication were 4 inches $(100 \mathrm{~mm})$ in diameter and $500 \mu \mathrm{m}$ in average thickness. RCA clean was performed before loading wafers into the furnace. The next step was thermal oxidation for growing a $2 \mu \mathrm{m}$ thick silicon dioxide on the top of the silicon wafer. A wet oxidation method was used. The process was conducted at $1075^{\circ} \mathrm{C}$ for ten hours.

Five points were measured to determine the deposited film thickness. The locations of the five points are shown in Figure 3.1. The film thickness was represented by the average of the five measured values. For a $2 \mu \mathrm{m}$ thick oxide film, the uniformity was typically about $0.1 \%$ with a standard deviation less than $10 \AA$.

The wafers were loaded in the LPCVD furnace for polysilicon deposition right after the thermal oxidation process. The polysilicon film was deposited at $617{ }^{\circ} \mathrm{C}$ using $\mathrm{SiH}_{4}$ gas with a typical deposition rate of $95 \AA / \mathrm{min}$ for a target thickness of $2.5 \mu \mathrm{m}$. Then, the polysilicon film was annealed at $1100{ }^{\circ} \mathrm{C}$ for one hour to form a fine-grained polycrystalline microstructure. For up to $3 \mu \mathrm{m}$ thick polysilicon films, the uniformity varied form $0.1 \%$ to $0.3 \%$. 


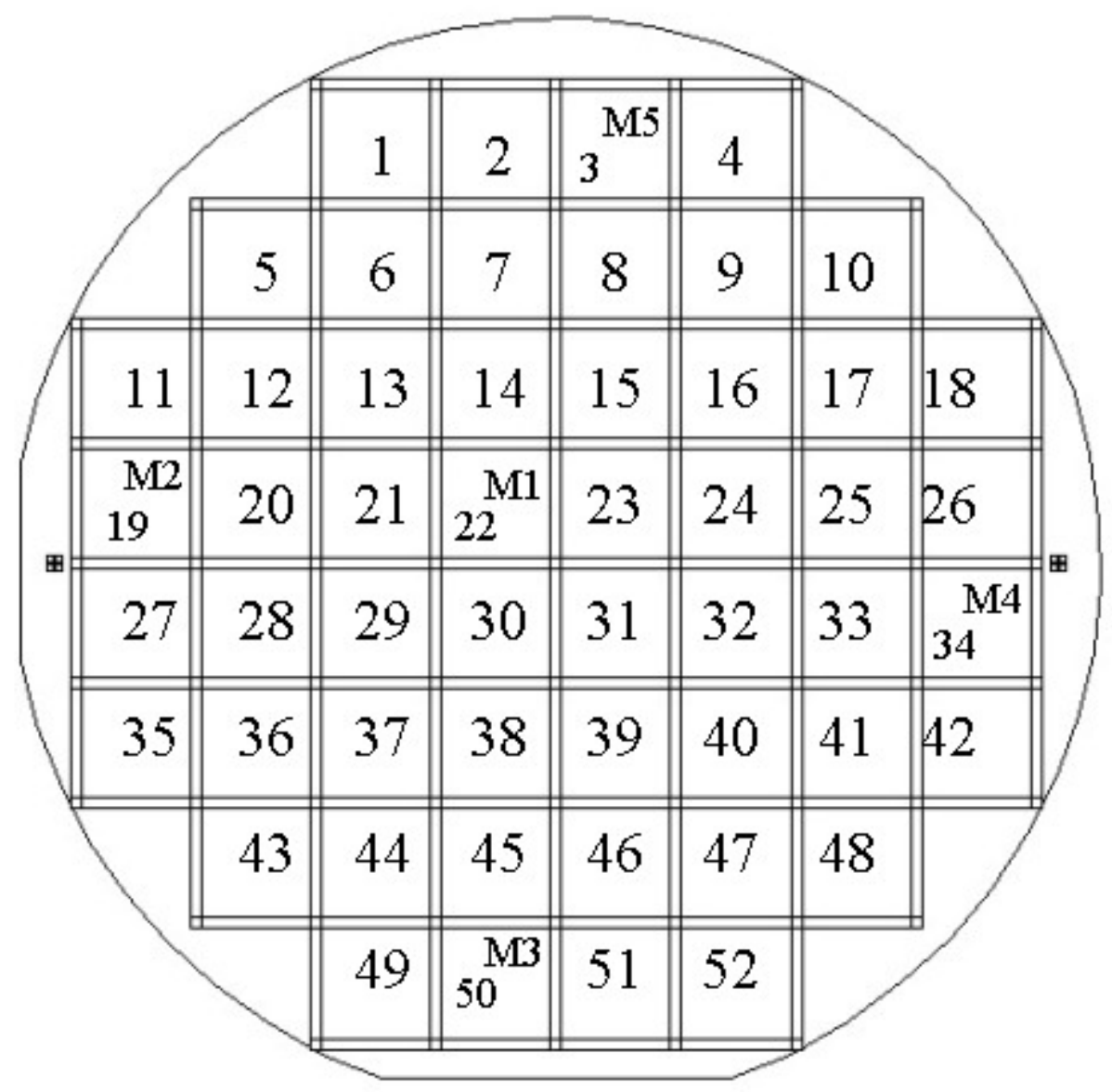

Figure 3.1 Locations of the five measuring points, M1 M5.

Mask 'odd layer' was used for digging trenches in the polysilicon film. First, $1.5 \mu \mathrm{m}$ thick photoresist film was applied on the top of the polysilicon film by setting the spinner speed and spinning time. Then, the photoresist film was covered by mask 'odd layer' and exposed under ultra-violet light. The following developing process removed the photoresist in the exposed areas and formed the etching mask for the next step. The top view of a typical trench area is shown in Figure 3.2. 


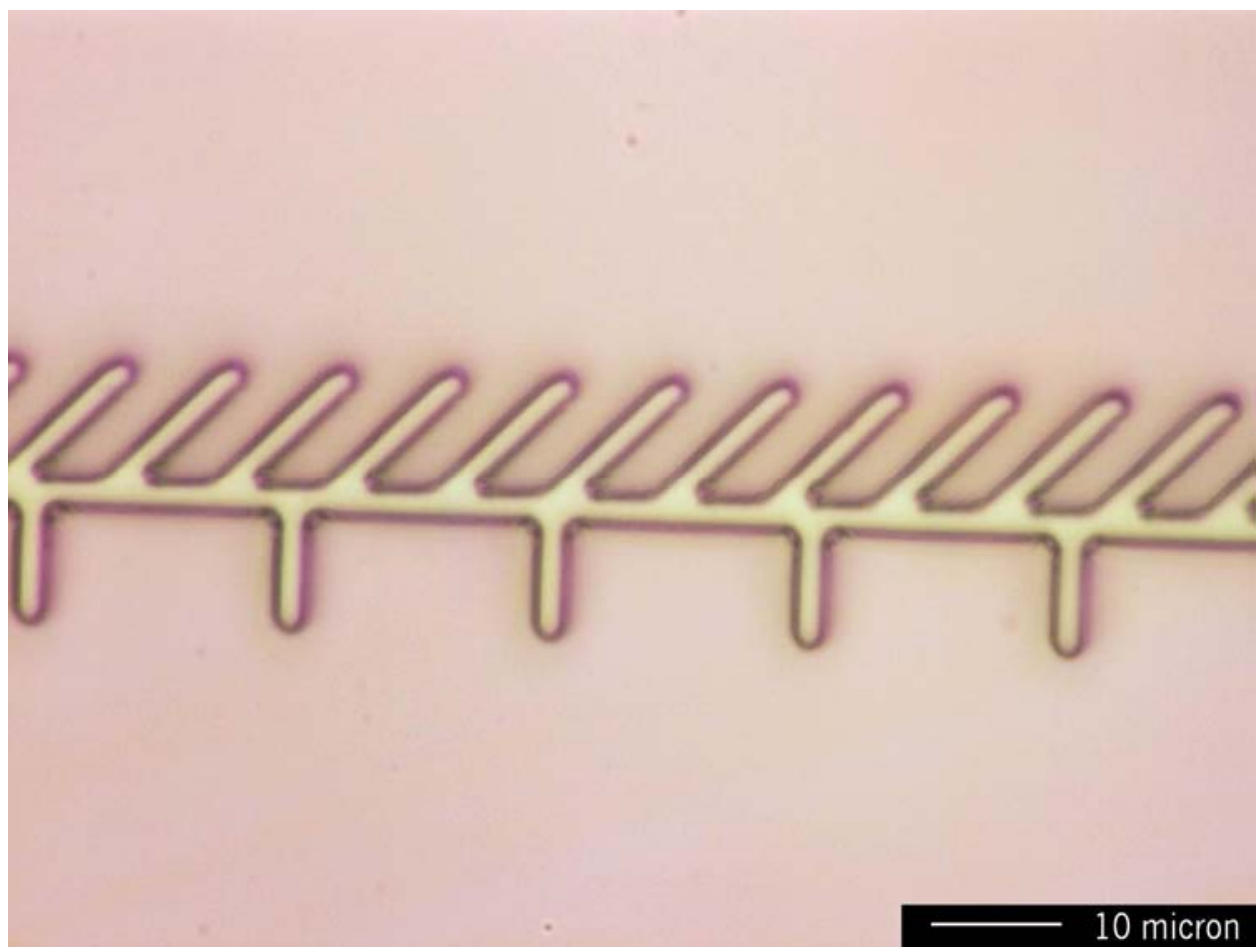

Figure 3.2 Top view of the photoresist etching mask with trenches.

The standard RIE recipe was used for etching the polysilicon in LAM490. Chlorine plasma was used to etch the polysilicon film anisotropically. The flow rate was $80 \mathrm{sccm}$ and $120 \mathrm{sccm}$ for Chlorine and the carrier gas Helium, respectively. The chamber pressure was $400 \mathrm{mT}$ Torr with a RF power of 200 watts during the etching process. The typical etch rate was about $3500 \AA / \mathrm{min}, 1824 \AA / \mathrm{min}$ and $150 \AA / \mathrm{min}$ for polysilicon, photoresist and silicon dioxide, respectively. After each etching process, step heights of the $20 \mu \mathrm{m}$ wide trenches were measured using a DEK-TEK stylus profilometer to determine the actual etch rate. For the first run, the polysilicon film was etched for two minutes and the polysilicon film was turned rough and black in the etched areas as shown in Figure 3.3. 


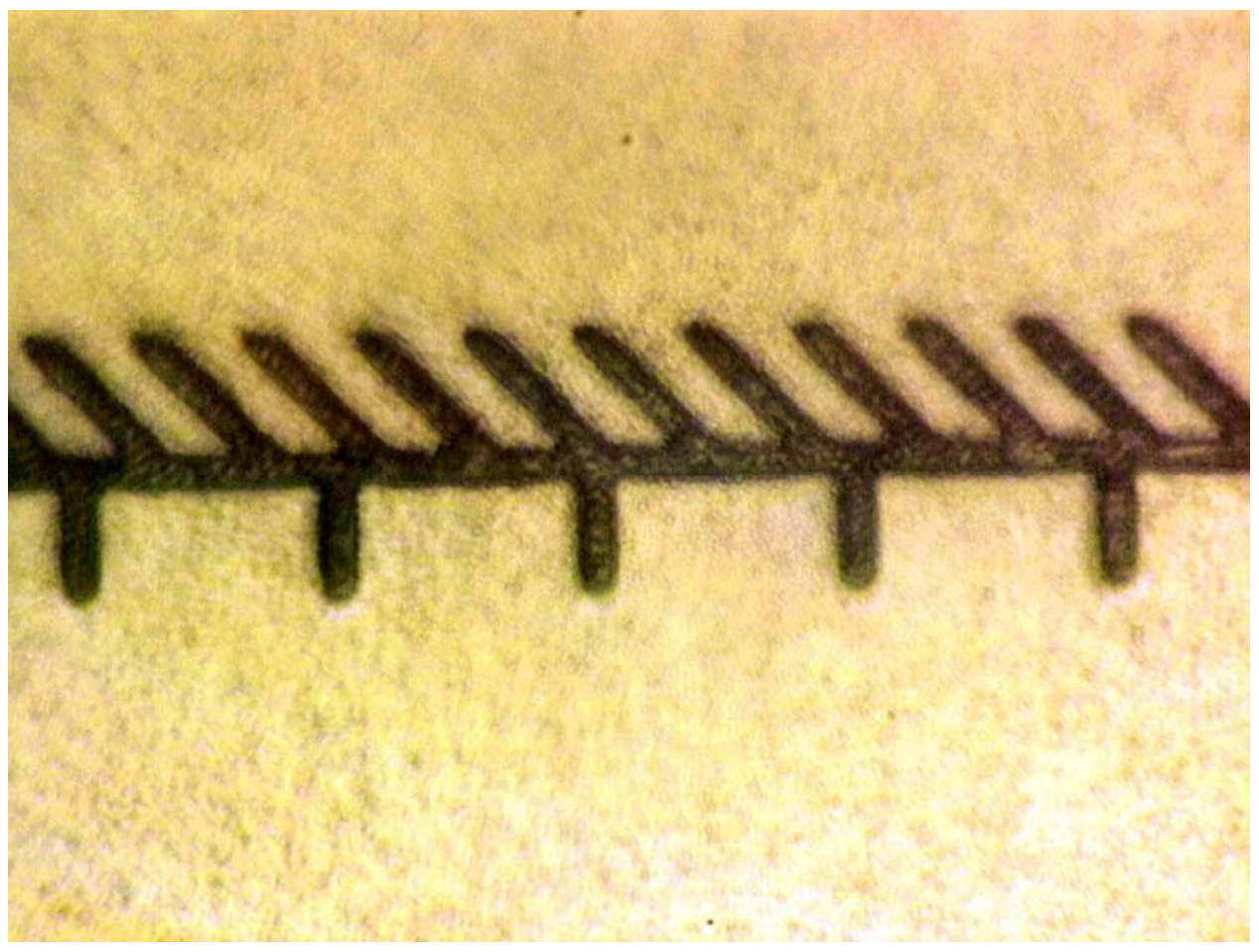

Figure 3.3 Black silicon in the etched trench area (polysilicon films, chlorine-based recipe, and photomask 'odd layer').

The step heights of the $20 \mu \mathrm{m}$ wide trenches were measured and listed in the second row of Table 3.1. Based on the standard etch rate of $3000 \AA / \mathrm{min}$ and $1600 \AA / \mathrm{min}$ for polysilicon and photoresist, the step heights were much smaller than what they should be.

\begin{tabular}{|l|c|c|c|c|c|}
\hline & $\mathrm{M} 1(\AA)$ & $\mathrm{M} 2(\AA)$ & $\mathrm{M} 3(\AA)$ & $\mathrm{M} 4(\AA)$ & $\mathrm{M} 5(\AA)$ \\
\hline After 2-minute etching & 15897 & 15104 & 14998 & 16135 & 15767 \\
\hline After 4-minute etching & 13054 & 12735 & 12543 & 13456 & 12892 \\
\hline $\begin{array}{l}\text { After removing photoresist } \\
\text { mask }\end{array}$ & 2533 & 2809 & 2601 & 2247 & 3041 \\
\hline
\end{tabular}

Table 3.1 Etching results for the polysilicon film with the chlorine-based recipe and the photomask 'odd layer'. 
The wafer was loaded into LAM for another 2 minutes etching. As shown in the third row of the Table 3.1, the step heights were even lower than before, which meant that the etch rate of the photoresist mask was higher than that of the polysilicon, or the black material appeared before it completely prevented the polysilicon from being further etched.

In order to determine how much polysilicon had been etched, the photoresist mask was removed using photoresist asher. The measurements showed that polysilicon was etched less than $3000 \AA$ within 4 minutes. This proved that the black material stopped the polysilicon-etching process.

The appearance of the black material is most likely caused by two possible issues. One possibility is that the etched photoresist redeposited on the substrate. This could have happened once the chamber became dirty and the residual product could not pump out immediately. The second possibility is the small exposed etching area. For the trench etching process, the open area was less than $1 \%$ of the total wafer area. The etching particles may not have been able to continuously contact with the target material due to the small open area. The small open area would also make the redeposition effect more critical to this etching problem.

In order to prove the explanation described above, mask 'final structure' was used to form the final microstructure patterns. Over $97 \%$ of the total wafer area was exposed to etchant with this mask. Patterned wafer with $2.5 \mu \mathrm{m}$ thick polysilicon film was etched for four minutes. The remaining thickness of the polysilicon film was measured using Nanospec4000. The average thickness after etching was $13223 \pm 632 \AA$. The corresponding etch rate was $2944 \AA / \mathrm{min}$, which agreed with the standard polysilicon etch rate. 
In Figure 3.4, no black material appears in the etched area around the anchor. Therefore, the small open area is one explanation for the appearance of the black material.

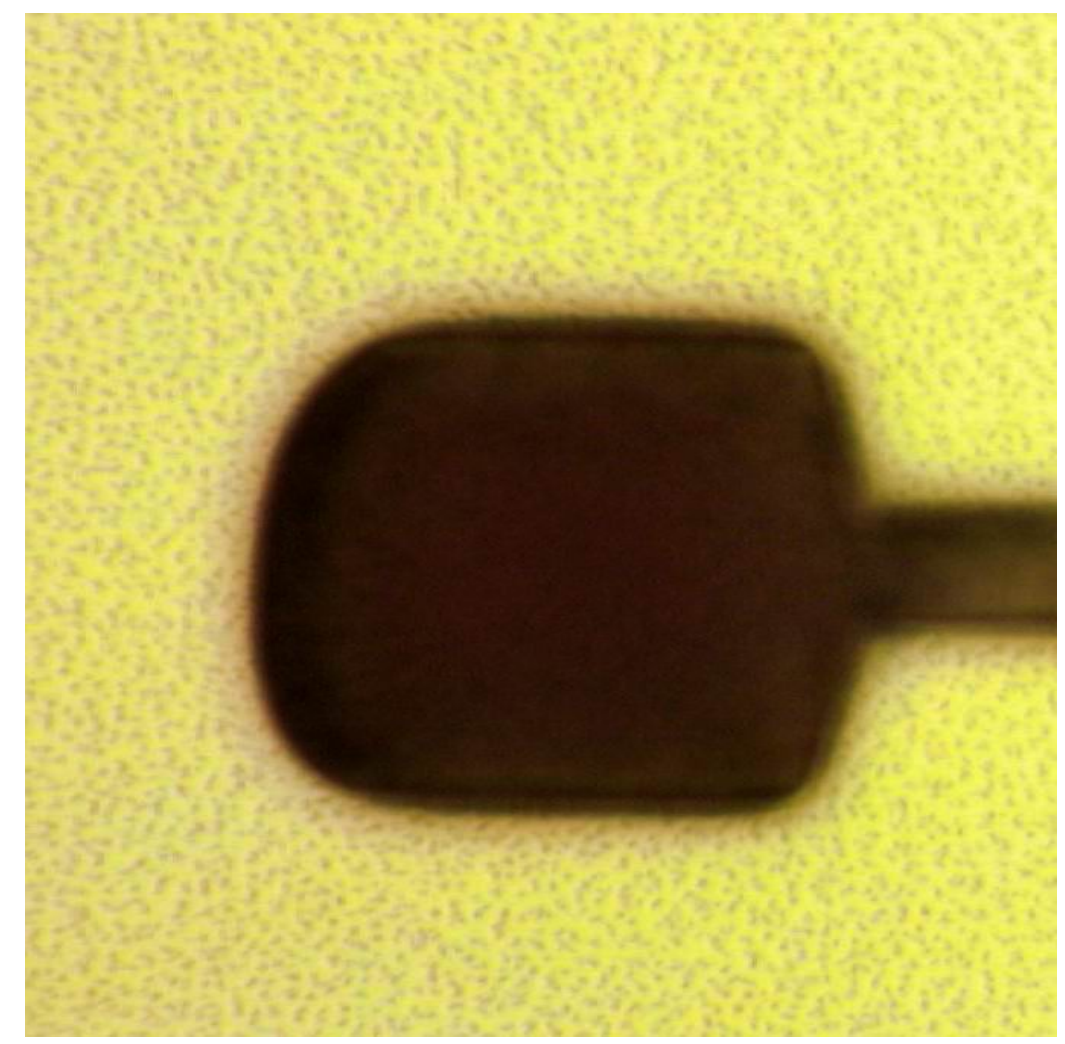

Figure 3.4 Etched areas around the anchor (chlorine-based recipe and photomask 'final structure').

The results presented above imply that the etch rate generated by the chlorine plasma is not fast enough for the etching products to be fully volatilized. This can be solved by using deep RIE spices such as $\mathrm{SF}_{6}$ and $\mathrm{O}_{2}$. It is necessary to obtain the etching results for the sputtered silicon using the same recipe before determining whether the chlorine-based recipe needs to be modified or a new recipe needs to be developed. 


\subsection{Sputtered silicon film}

\subsubsection{Sputtering tool and silicon target}

The sputtering machine is Discover-24LL (Denton Vacuum, Inc.) featured with four DC magnetron cathodes. The silicon target used for all of the sputtering processes is an N-type doped round silicon plate with a purity of $99.999 \%$. Its diameter and thickness are 3 inches and 0.25 inches, respectively. The concentrations of impurity elements are listed in Table 3.2 in the unit of ppm (Parts Per Million).

\begin{tabular}{|c|c|c|c|c|c|c|}
\hline Element & $\mathrm{Al}$ & $\mathrm{B}$ & $\mathrm{C}$ & $\mathrm{Fe}$ & $\mathrm{P}$ & $\mathrm{Pb}$ \\
\hline Concentration $(\mathrm{ppm})$ & 0.05 & 0.05 & 3 & 0.001 & 0.05 & 0.001 \\
\hline
\end{tabular}

Table 3.2 Impurity concentrations in silicon target.

\subsubsection{Deposition rate and residual stresses}

The silicon target is brittle material, and it is very easy to break if the power increases from zero to a relatively high value within a very short time. A feasible operation is to increase the power within several periods. First, slowly ramp the power to a quarter of the target value and keep it for 5 minutes to make it stable. Then, do the same thing for half, three-quarters and finally for the full target value. During the stabilizing period, the shutter is closed so that the sputtered particles cannot travel to the wafer surface.

The first attempt was done with 5mTorr of pressure and 200Watts of power. The testing wafer was prepared with $2 \mu \mathrm{m}$ thick thermal oxide film. The total sputtering time was 120 minutes and the chamber temperature was $25{ }^{\circ} \mathrm{C}$ during the sputtering process. 
The thickness of the sputtered silicon film was measured using Nanospec4000. The measuring results varied greatly across the wafer, and the fit between the sample curve and the model curve was very bad. This meant that the sputtered silicon film had a different crystal structure from the model material or had no continuous crystal structure. Therefore, the measured thickness from Nanospec 4000 was not accurate and could not be used for determining the deposition rate of the sputtered silicon.

One way to acquire thickness is to attach partially covered glass plates onto the wafer stage at the locations around the wafer. During the sputtering process, the silicon film is also sputtered on the partially covered glass plates. After the process is done, the covers are removed and the step heights between the covered area and the exposed area are measured. The mean of the measured step height is just the thickness of sputtered silicon film.

For the actual operation, four glass plates were used and distributed around the wafer with an equally spaced manner. After the sputtering process, five measurements were collected from each of the glass plates and the mean of these measured data was considered as the thickness of the sputtered silicon film.

A test was set to detect whether the deposition thickness linearly varied with respect to the deposition time. This test was comprised of three continuous depositions with deposition times of 120 minutes, 150 minutes and 180 minutes, respectively. The power and the pressure were still 200Watts and 5mTorr.

Measuring data and the corresponding deposition rates are listed in Table 3.3. The deposition rate is consistent with various deposition times. The deposition rate is relatively high in the beginning phase and tends to stabilize with the increment of 
deposition time. For the processes in this thesis, the target film thickness is always greater than $2 \mu \mathrm{m}$, thus the stable deposition rate can be employed to estimate the processing time needed for sputtering silicon film.

\begin{tabular}{|c|c|c|}
\hline Deposition time (minute) & Film thickness $(\AA)$ & Deposition rate $(\AA / \mathrm{min})$ \\
\hline 120 & $15338 \pm 217$ & 129 \\
\hline 150 & $18235 \pm 229$ & 122 \\
\hline 180 & $21344 \pm 353$ & 119 \\
\hline
\end{tabular}

Table 3.3 Deposition rates of the sputtered silicon film using Discover24.

Residual stresses in thin films play a very important role in determining the mechanical properties of the released microstructures. For a double-clamped beam, too large a compressive residual stress will cause the beam to buckle and too large a tensile residual stress will make the structure too stiff and possibly crack. Formula 3.1 estimates the critical buckling stress with respect to the structure thickness and length.

$$
\sigma_{c r}=E \frac{\pi^{2} t^{2}}{3 l^{2}}
$$

Here, $\mathrm{E}$ is the Young's modulus; $\mathrm{t}$ and 1 are the beam thickness and length, respectively. For the longest microstructure designed in Chapter 2, which is $300 \mu \mathrm{m}$, and released from a $2 \mu \mathrm{m}$ thick silicon film, the critical compressive stress needed to make the microstructure buckle is only $23 \mathrm{MPa}$. So the compressive residual stress has to be avoided in the sputtered silicon film. Generally, the tensile stress that is lower than $100 \mathrm{MPa}$ is acceptable for the applications in this thesis. 
Since the thermal annealing process cannot be conducted after sputtering the silicon film, the only way to further lower the residual stresses is to adjust the processing power and pressure. The processing power and pressure also influence the deposition rate.

Trial experiments were arranged to study the influence of the processing parameters. Considering the usage frequency of the sputtering tool and the slow deposition rate, only four cases were studied with the power and pressure combinations shown in the first column of Table 3.4.

The deposition time was fixed to 180 minutes for all of the four processes. All of the sputtered silicon films were deposited on the top of thermally grown oxide films with a thickness of $2 \mu \mathrm{m}$. The residual stress was measured using the film stress measurement tool, which can calculate the residual stress in the thin film by precisely measuring the change of the wafer curvature. The curvature map and the calculated residual stress are shown in Figure 3.5 for the sputtered silicon film deposited with 200Watts of power and $5 \mathrm{mTorr}$ of pressure. 
$8 / 8 / 103$

C:IFSMULXC51ISPU_SI.MPH

Slot No:0

Film Stress: $60.45 \mathrm{MPa}$

Curvature Radius: $68.85 \mathrm{~m}$

Wafer Center Height: -6.39um

〈LASER810〉

[TENSILE]

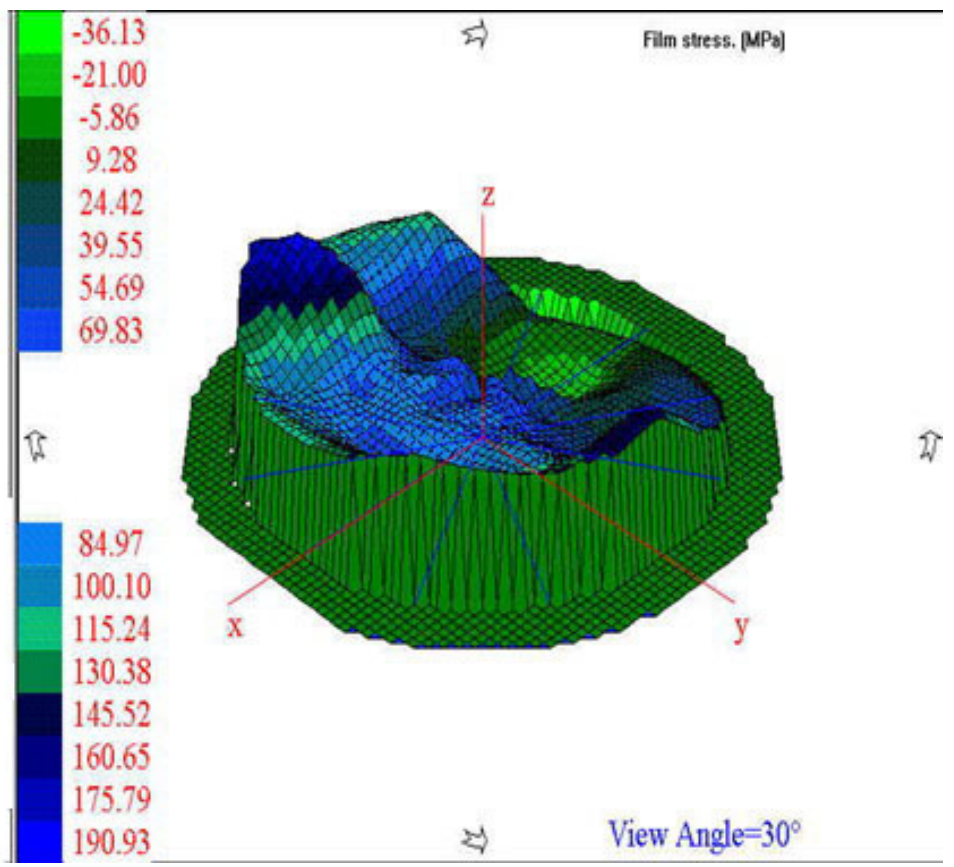

Figure 3.5 Curvature map and calculated residual stress for the sputtered silicon film deposited with 200Watts of power and 5mTorr of pressure.

\begin{tabular}{|c|c|c|c|}
\hline $\begin{array}{c}\text { Power, pressure } \\
(\text { Watts, } m \text { Torr })\end{array}$ & Thickness $(\mu \mathrm{m})$ & $\begin{array}{c}\text { Deposition } \\
\text { rate }(\AA / \mathrm{min})\end{array}$ & $\begin{array}{c}\text { Residual stress } \\
(\mathrm{MPa})\end{array}$ \\
\hline 200,5 & 22012 & 122 & 60.45 \\
\hline 300,5 & 27740 & 154 & 47.35 \\
\hline 200,10 & 18642 & 104 & 101.08 \\
\hline 300,10 & 25264 & 140 & 107.62 \\
\hline
\end{tabular}

Table 3.4 Deposition rates and residual stresses for the sputtered silicon films using different process parameters with Discover24.

The residual stresses and the relevant deposition rates are listed in Table 3.4. It is very clear that the deposition rate turns faster when the power increases. Oppositely, the higher pressure makes the deposition rate decrease.

The residual stresses are tensile stresses for the four cases. The higher pressure generates higher tensile stress, which is greater than 100MPa. The effect of the power on the residual stresses is not clear since higher power makes the tensile stress more notable 
with $10 \mathrm{mT}$ Torr of pressure and lowers the tensile stress with 5mTorr of pressure. The tensile stresses are smaller than 100MPa with 5mTorr of pressure. This makes it possible to carry out the sputtering process without worrying about how to release the residual stresses in the sputtered silicon film.

In terms of the comparison of the data in Table 3.4, the combination of 300 Watts and $5 \mathrm{mT}$ Torr is the best configuration for sputtering silicon in the sense of obtaining lower residual stress. However, high power always increases the risk of breaking the silicon target, which is a costly and time-consuming problem. Since the combination of 200Watts and 5mTorr can also produce good silicon film with enough low residual stress, it is acceptable to choose this option even though the deposition rate has to be sacrificed. Therefore, the sputtering parameters will be fixed to 200 Watts of power and $5 \mathrm{mT}$ Torr of pressure for latter processes.

\subsubsection{Surface roughness}

Surface roughness is one of the important parameter for assessing the quality of sputtered thin films. It can be measured using an atomic force microscope (AFM) or a nanoindentation system combined with AFM or SPM.

Tapping mode AFM was employed for studying the surface roughness of the sputtered silicon films. Figure 3.6 shows a typical AFM graph for the surface topography of the sputtered silicon film. The testing results for three films are shown in Table 3.5. The testing samples were small chips located in the wafer center. For each sample, five locations were measured and the mean was used to represent the surface roughness. Table 3.6 presents the mean values for three tested films. 


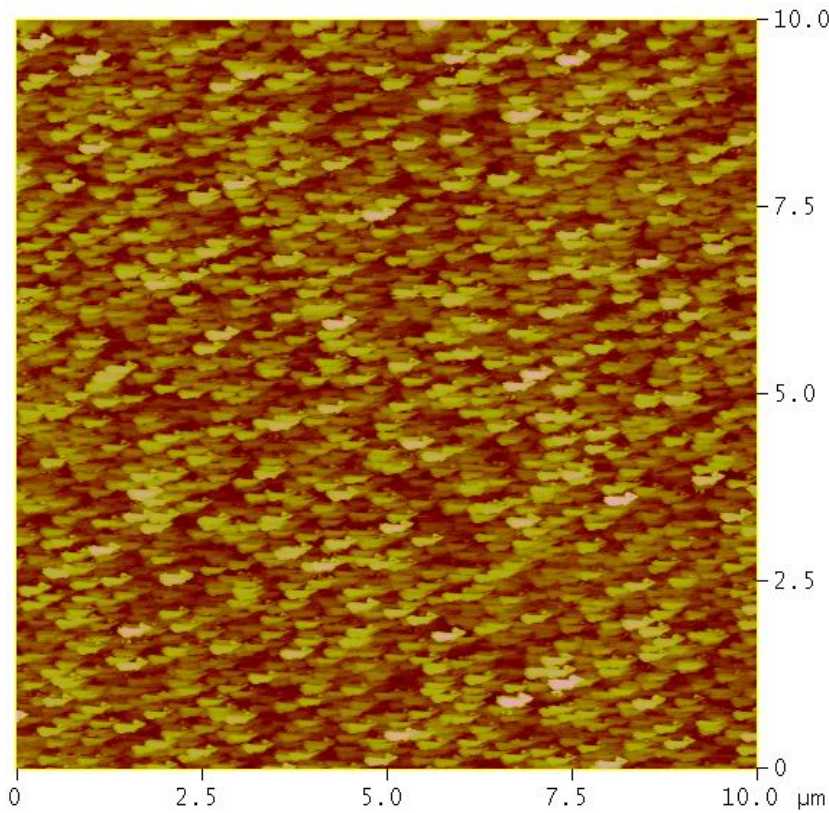

\begin{tabular}{|ll|}
\multicolumn{3}{c}{ Image Statistics } \\
\hline Img. Z range & $13.222 \mathrm{~nm}$ \\
Img. Raw mean & $-0.000005 \mathrm{~nm}$ \\
Img. Rms (Rq) & $1.485 \mathrm{~nm}$ \\
Img. Ra & $1.171 \mathrm{~nm}$ \\
Img. Rmax & $13.222 \mathrm{~nm}$ \\
\hline
\end{tabular}

\begin{tabular}{|l|}
\multicolumn{1}{|c|}{ Box Statistics } \\
\hline Rms $(\mathrm{Rq})$ \\
Box $\times$ dimension \\
Box $\mathrm{y}$ dimension \\
\hline
\end{tabular}

1c082703.f00

Peak off

Figure 3.6 Surface roughness of the sputtered silicon film with thickness of $15338 \AA$ (tapping mode AFM).

\begin{tabular}{|c|c|}
\hline Film Thickness $(\AA)$ & RMS Roughness $(\mathrm{nm})$ \\
\hline 15338 & $1.2 \pm 0.2$ \\
\hline 18235 & $1.5 \pm 0.1$ \\
\hline 21344 & $2.2 \pm 0.3$ \\
\hline
\end{tabular}

Table 3.5 Surface roughness of sputtered silicon films.

The results in Table 3.5 indicate the roughness increases when the film turns thicker. The roughness is relatively smaller than that reported by Honer ${ }^{[20]}(3.1 \pm 0.8 \mathrm{~nm}, 1500$ Watts, 14 mTorr). 
. The reason is that the pressure and power used for the sputtering process are lower than those Honer used. This results in a lower deposition rate of $122 \AA / \mathrm{min}$ compared to the minimum deposition rate of $190 \AA / \mathrm{min}$ achieved in their processes. The lower deposition rate reflects a stable nucleation and coalescence procedure and a better quality film with reduced roughness.

\subsubsection{Sputtered silicon material characterization}

Before the sputtering process, the sputtering chamber is pumped down to lower pressure to generate a high vacuum environment. The best vacuum the sputtering tool can achieve is $10^{-6}$ Torr. This pressure is not low enough to pump out all species from the chamber. Thus, oxygen or other elements may incorporate in the sputtered silicon film and affect the latter fabrication processes. Element analysis can determine the element components and their percentage rate in the sputtered silicon film.

The element analysis was done by means of X-ray Photo-electron Spectrometry (XPS). A Perkin-Elmer PHI-5600 system was used to analyze the depth profiles by sputtering inert gas source onto sample films. The wafer with $21344 \AA$ thick sputtered silicon film was used for this analysis and only the top $5000 \AA$ deep film was analyzed.

The element profiles are shown in Figure 3.7. The atomic concentration of oxygen increases from $5 \%$ to $8 \%$ corresponding to the depth variance from $50 \AA$ to $450 \AA$, and then slowly drops to $2 \%$ when the depth reaches $2500 \AA$ and keeps this concentration all the way down to the depth of $5000 \AA$. The oxygen concentration in the first $100 \AA$ deep film can be explained as the native oxidation caused when the wafer is exposed to the air. The relatively high concentration within the rest of the film is definitely due to the unclean sputtering chamber. The only way to solve this problem is to pump the sputtering 
chamber for a longer time. Actually, this has been practiced many times and the best result is $10^{-7}$ Torr. This vacuum status will not significantly improve the impurity problem. Therefore, the sputtered silicon film with oxygen impurity has to be used for the microstructure and extra effort is needed to solve problems that might occur in the latter etching process.

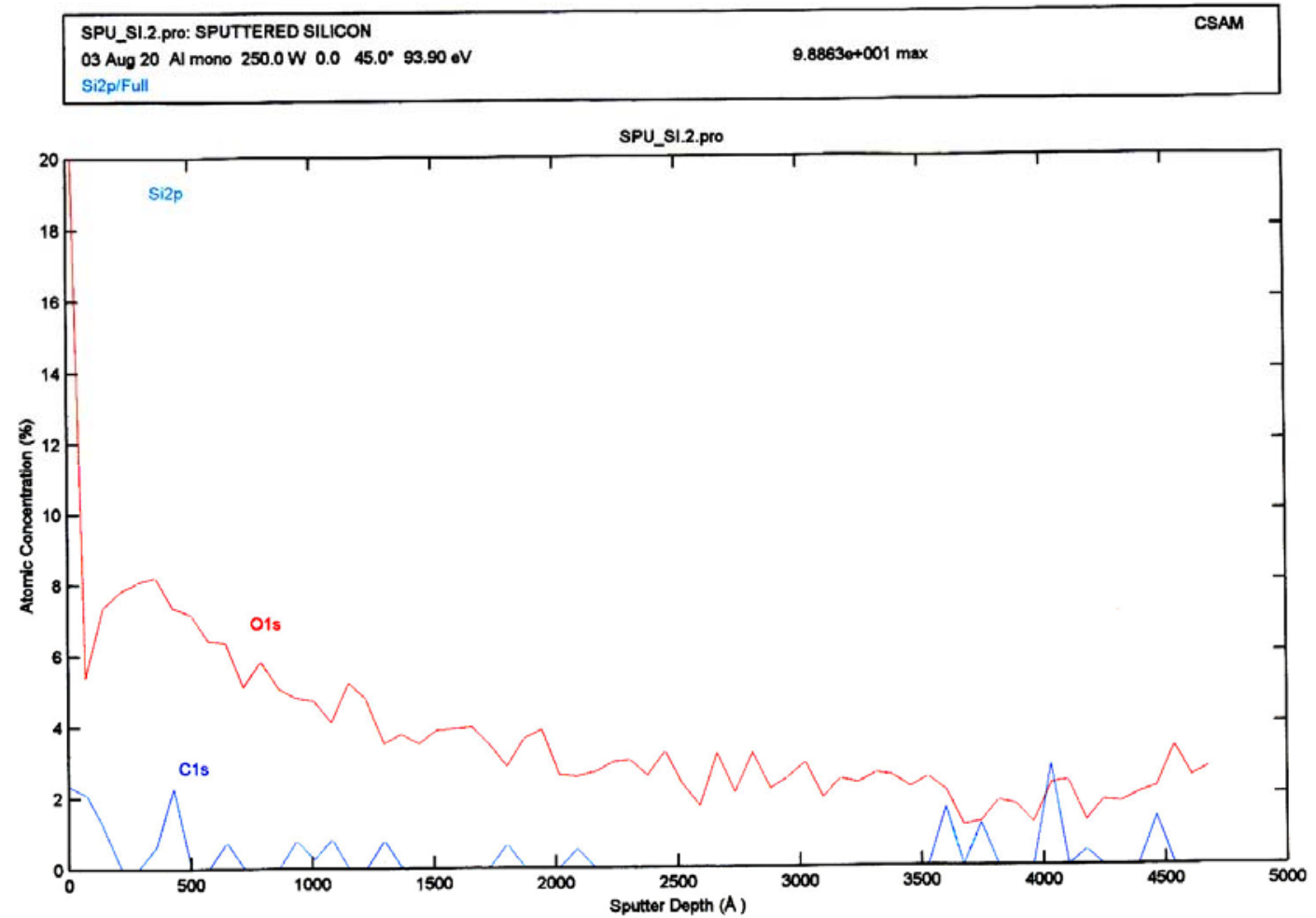

Figure 3.7 Element profiles in the sputtered silicon film.

Due to the nature of the sputtering process, the sputtered silicon is expected to be amorphous. This can be testified by means of X-ray Diffraction (XRD).

A Scintag X-1 advanced X-ray diffractometer was used for the XRD measurements. A standard measurement data for face-centered single crystal silicon was used for determining the major peak positions. The first major peak appears at $28.5^{\circ}$ and 
the other two major peaks appear within $65^{\circ}$. So the scan range of the detector was set to be $25^{0} \sim 65^{0}$ with an increment step of $0.03^{0}$.

In order to compare the crystal structures of the polysilicon film and the sputtered silicon film, the polysilicon film obtained using LPCVD was also scanned. Figure 3.8 and Figure 3.9 show the scanning results for both of the films. The scanning data for the sputtered silicon film has no major peaks, which means that no periodic crystal structures exist. As expected, three major peaks are clearly indicated in the polysilicon scan. The XRD study proves that the sputtered silicon has no periodic crystal structures and is amorphous.

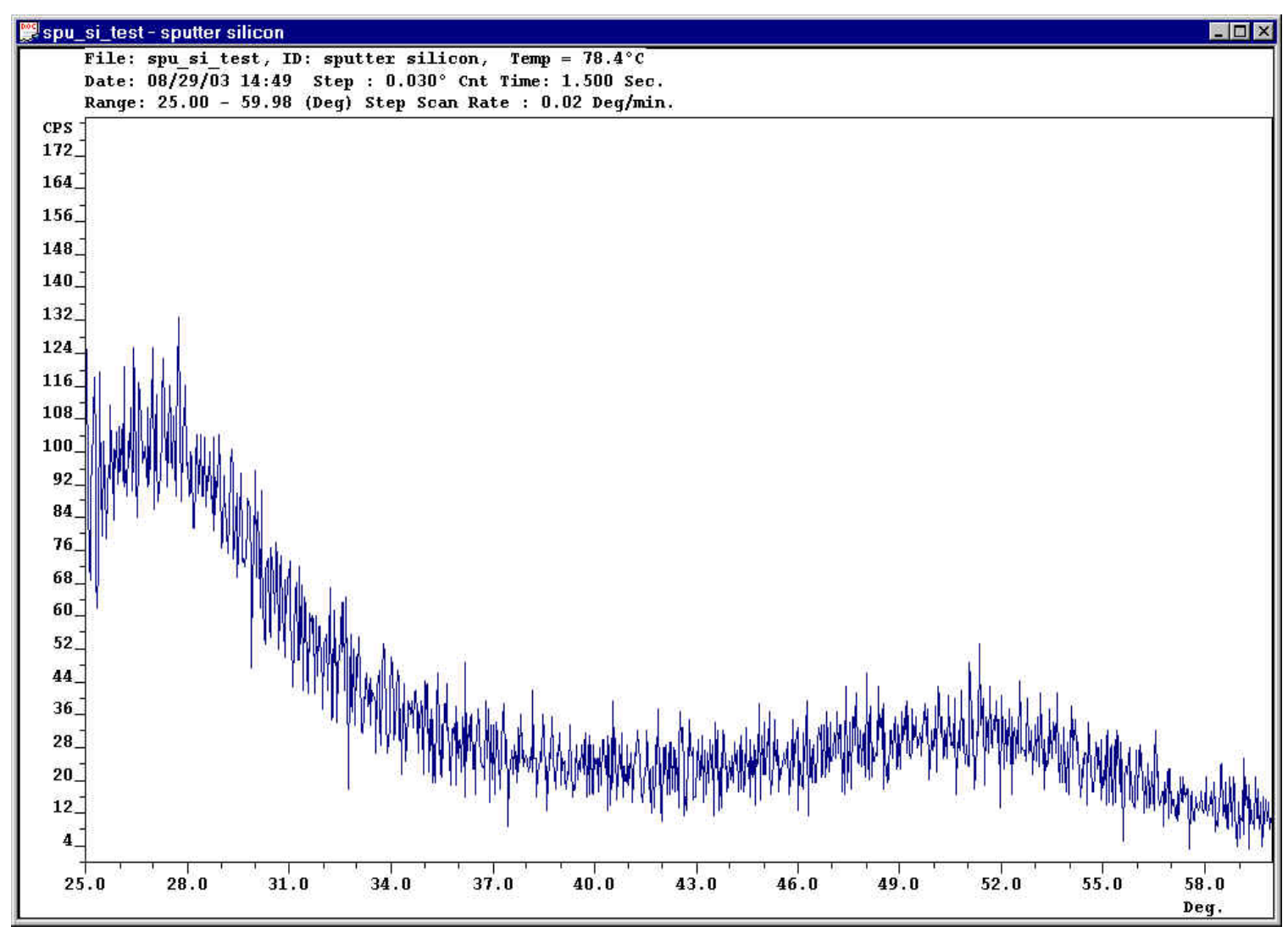

Figure 3.8 X-ray diffraction pattern of the sputtered silicon film. 


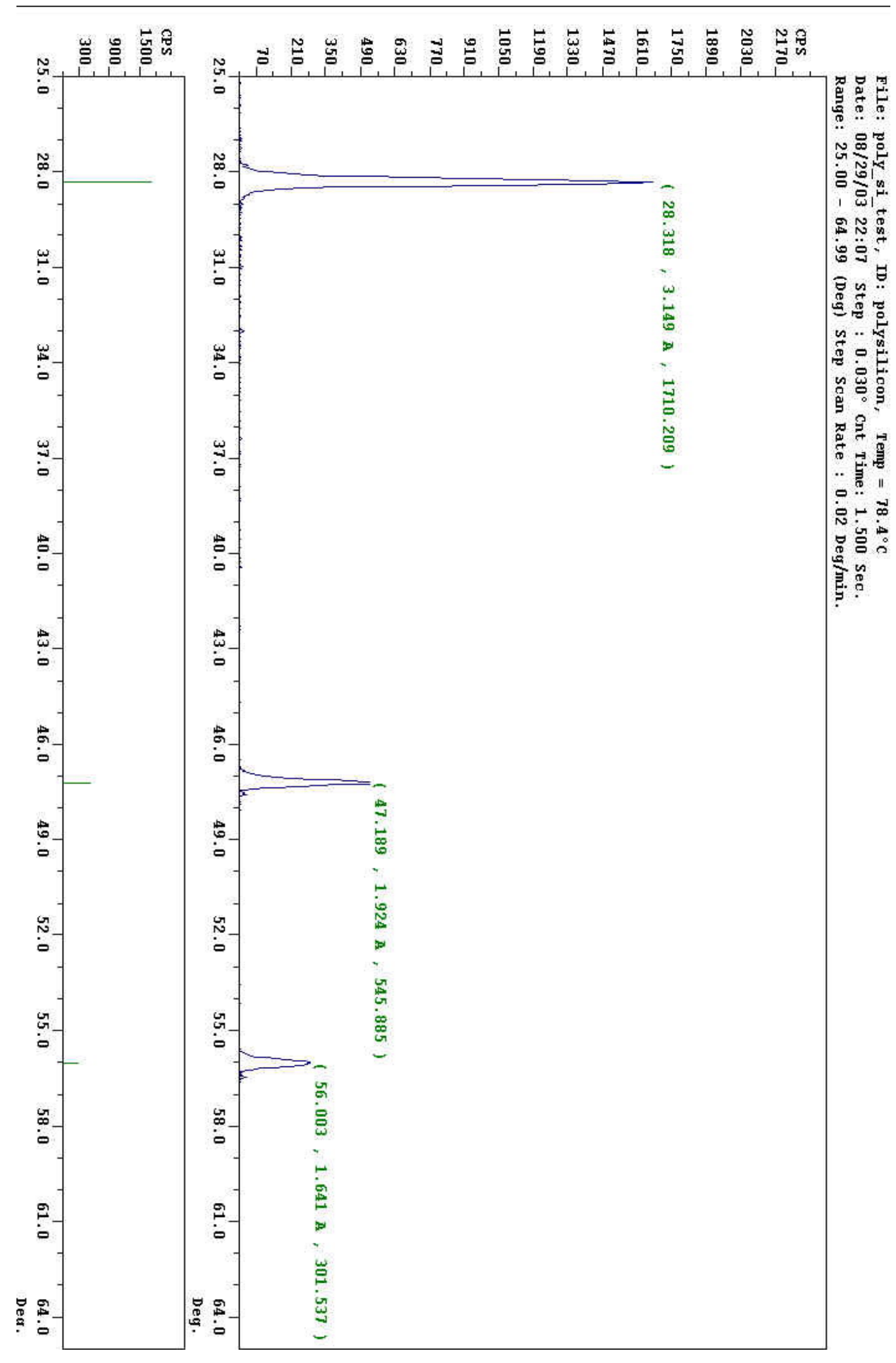

Figure 3.9 X-ray diffraction pattern of the polysilicon film. 


\subsubsection{Etching sputtered silicon films}

When using chlorine-based plasma to etch the polysilicon film, the black silicon will appear in the etched area if the exposed area is only a small fraction of the whole wafer area. This phenomenon will not repeat if the exposed area is not relatively small compared to the wafer area. The etching study will also be conducted on the sputtered silicon film to further observe the etching behavior of the chlorine-based plasma for small and large exposed etching areas.

The wafer preparation was completely the same for etching the polysilicon film. The etching stop layer was still $2 \mu \mathrm{m}$ thick silicon dioxide film. The sputtering process used fixed pressure and power, which were 5mTorr and 200Watts, respectively. Sputtering time was set to be 180 minutes to reach a silicon film thickness of about $2 \mu \mathrm{m}$. Photomask 'Odd layer' was used for pattering the photoresist film, which was $1.2 \mu \mathrm{m}$ thick, to form the etching mask. The first time, the sputtered silicon film was etched for 2 minutes. The etching result is shown in Figure 3.10. The etched area turned black as the polysilicon etching. The step height measurements shown in the second row of Table 3.7 indicated that almost no sputtered silicon was etched based on the standard photoresist etch rate of $1800 \AA / \mathrm{min}$.

Then, the wafer was etched for another two minutes. The etched area was still black and the measuring data in the third row of Table 3.6 further proved that no sputtered silicon had been etched since the black silicon appeared. 


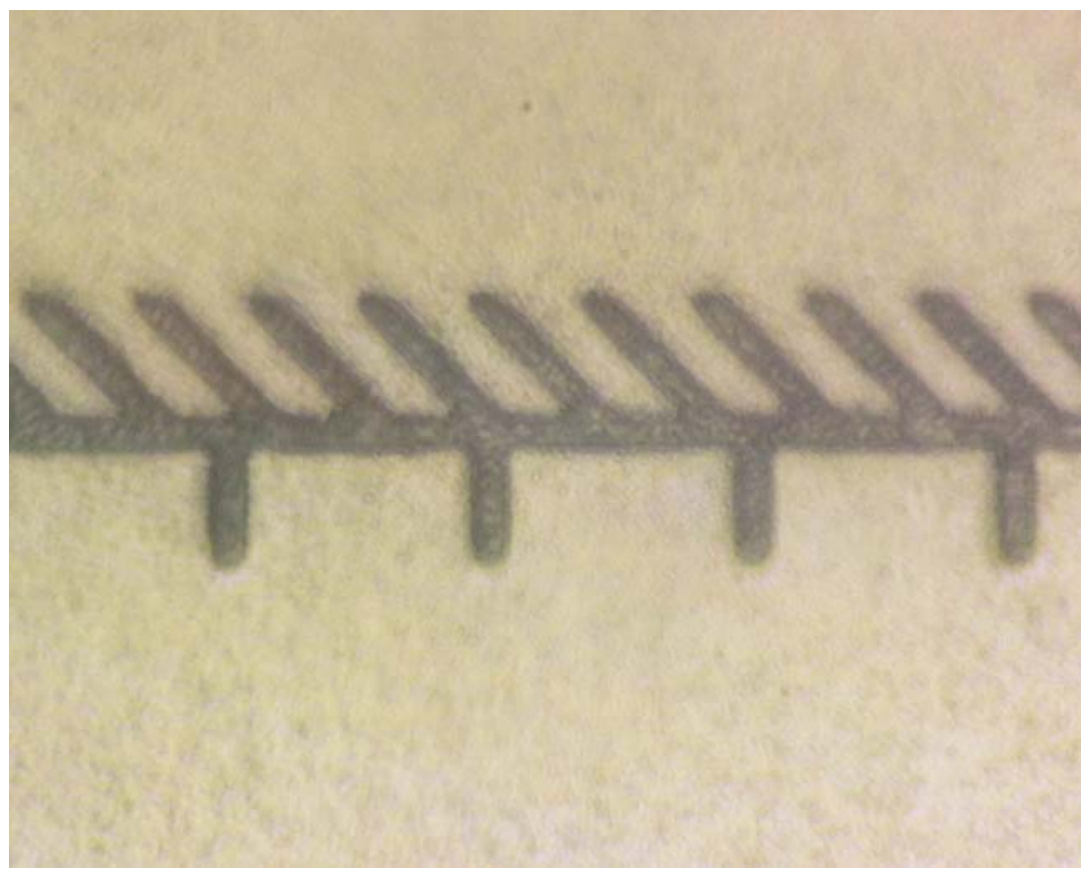

Figure 3.10 Black silicon in the etched area (sputtered silicon films, chlorine-based recipe, and photomask 'odd layer').

\begin{tabular}{|l|l|l|l|l|l|}
\hline & M1 $(\AA)$ & M2 $(\AA)$ & M3 $(\AA)$ & M4 $(\AA)$ & M5 $(\AA)$ \\
\hline After 2-minute etching & 9547 & 9874 & 10335 & 10241 & 9653 \\
\hline After 4-minute etching & 6152 & 5737 & 6489 & 7012 & 6988 \\
\hline $\begin{array}{l}\text { After removing photoresist } \\
\text { mask }\end{array}$ & 254 & 137 & 601 & 221 & 511 \\
\hline
\end{tabular}

Table 3.6 Etching results of the sputtered silicon film with chlorine-based recipe and photomask 'odd layer'.

After removing the photoresist mask, the actual etched depth was found to be about a few hundred angstroms and the etching profile was non-uniform across the wafer. Next, photomask 'final structure' was used to pattern the photoresist film and the wafer with patterned mask was loaded into LAM for 2 minutes. The top view of the area around one of the anchors after the etching process is shown in Figure 3.11. The sputtered silicon 
still turned black even though using the photomask with the large exposed area. The black silicon was even rougher and darker in this case.

Then, the same processes were carried out, namely measuring the step heights, etching the wafer for another 2 minutes, measuring the step heights, removing the photoresist mask and eventually measuring the step heights again. The testing data are shown in Table 3.7.

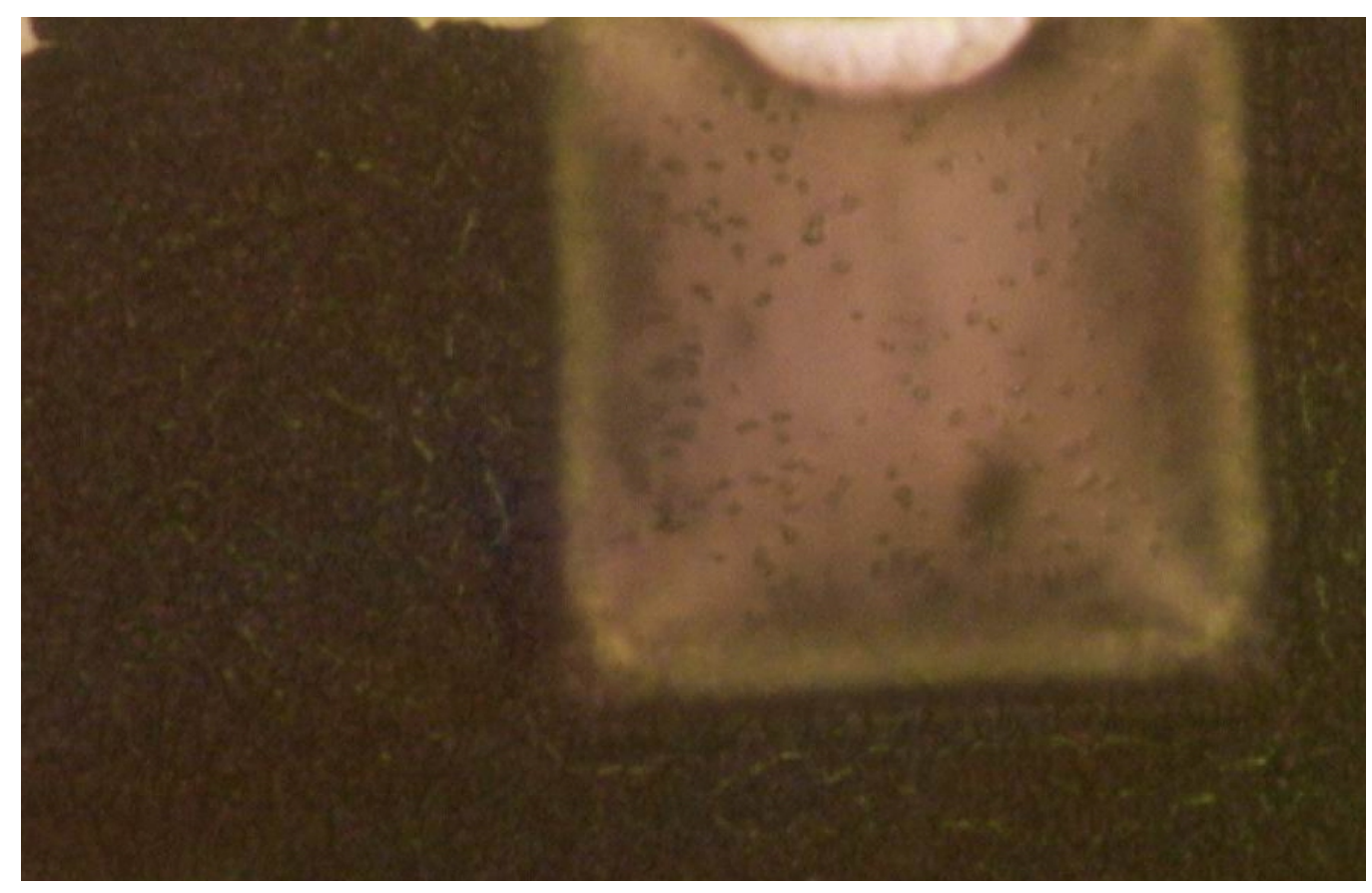

Figure 3.11 Black silicon around the anchor area (sputtered silicon film, chlorine-based recipe, and photomask 'final structure').

\begin{tabular}{|l|l|l|l|l|l|}
\hline & M1 $(\AA)$ & M2 $(\AA)$ & M3 $(\AA)$ & M4 $(\AA)$ & M5 $(\AA)$ \\
\hline After 2-minute etching & 8755 & 9233 & 9952 & 8374 & 9053 \\
\hline After 4-minute etching & 6011 & 7217 & 6328 & 5899 & 6988 \\
\hline $\begin{array}{l}\text { After removing photoresist } \\
\text { mask }\end{array}$ & 887 & 1059 & 722 & 564 & 982 \\
\hline
\end{tabular}

Table 3.7 Etching results of the sputtered silicon film with chlorine-based recipe and photomask 'final structure'. 
The study shown above indicates that the sputtered silicon cannot be etched with chlorine-based plasma. The black silicon produced with photomask 'odd layer' is due to the small exposed area, but the black silicon appeared after etching with photomask 'final structure' implies that there must be other reasons for making the sputtered silicon hardly etched. Recalling the element analysis results shown in Section 3.2.3, the impurity in the sputtered silicon is the most likely source that causes the black silicon. In the top $5000 \AA$ sputtered silicon profile, oxygen concentration is about $8 \%$ within the first few hundred angstroms, drops to $2 \%$ at the depth of $2500 \AA$ and keeps this level all the way down to the depth of $5000 \AA$. The oxygen concentration is relatively high in the sputtered silicon film compared to the polysilicon film deposited using LPCVD. The oxygen incorporated in the sputtered silicon film may form silicon dioxide. Even though the proportion of the oxide byproduct is small, it can play an important role as etching mask to prevent the chlorine-based plasmas from reacting with silicon to form volatile product. Thus, no silicon will be etched at all.

In order to solve the silicon etching problems, the exposed area in photomask 'odd layer' has to be enlarged and the impurity in the sputtered silicon film has to be removed. However, the current sputtered silicon film has already achieved the best quality that Discover24 can provide. There is almost no space to further improve it. On the other hand, the fabrication design does not allow the photomask 'odd layer' to have large exposed area. Otherwise, it will cause problems for depositing the second and the third structural layers. Therefore, new recipes have to be developed with the current configuration of LAM490. 
After studying the origin of the black silicon, a silicon etching recipe using $\mathrm{SF}_{6} / \mathrm{O}_{2}$ based plasma was developed. The detailed developing procedure is presented in Appendix A.2. Thereafter, photoresist related processes are discussed in Appendix A.3, A.4 and A.5.

\subsection{Successful microstructures}

Using the testing data and the tool parameters presented in Appendix A, microcomposite structures were successfully fabricated.

First, the wafer was thermally oxidized to grow $2 \mu \mathrm{m}$ thick oxide film. The first structural layer was $2.5 \mu \mathrm{m}$ thick polysilicon deposited using LPCVD. The actual thickness was $25076 \pm 77 \AA$. Trial recipe- 3 and photomask 'odd layer' were used to etch the polysilicon film. The wafer top view after this step is shown in Figure 3.12.

In order to keep the microstructure intact and prevent the photoresist from sticking on the substrate after the final releasing, the polysilicon film was not etched through the whole film depth. Thus, the interface between the microstructure and the substrate was still formed by polysilicon and oxide. 


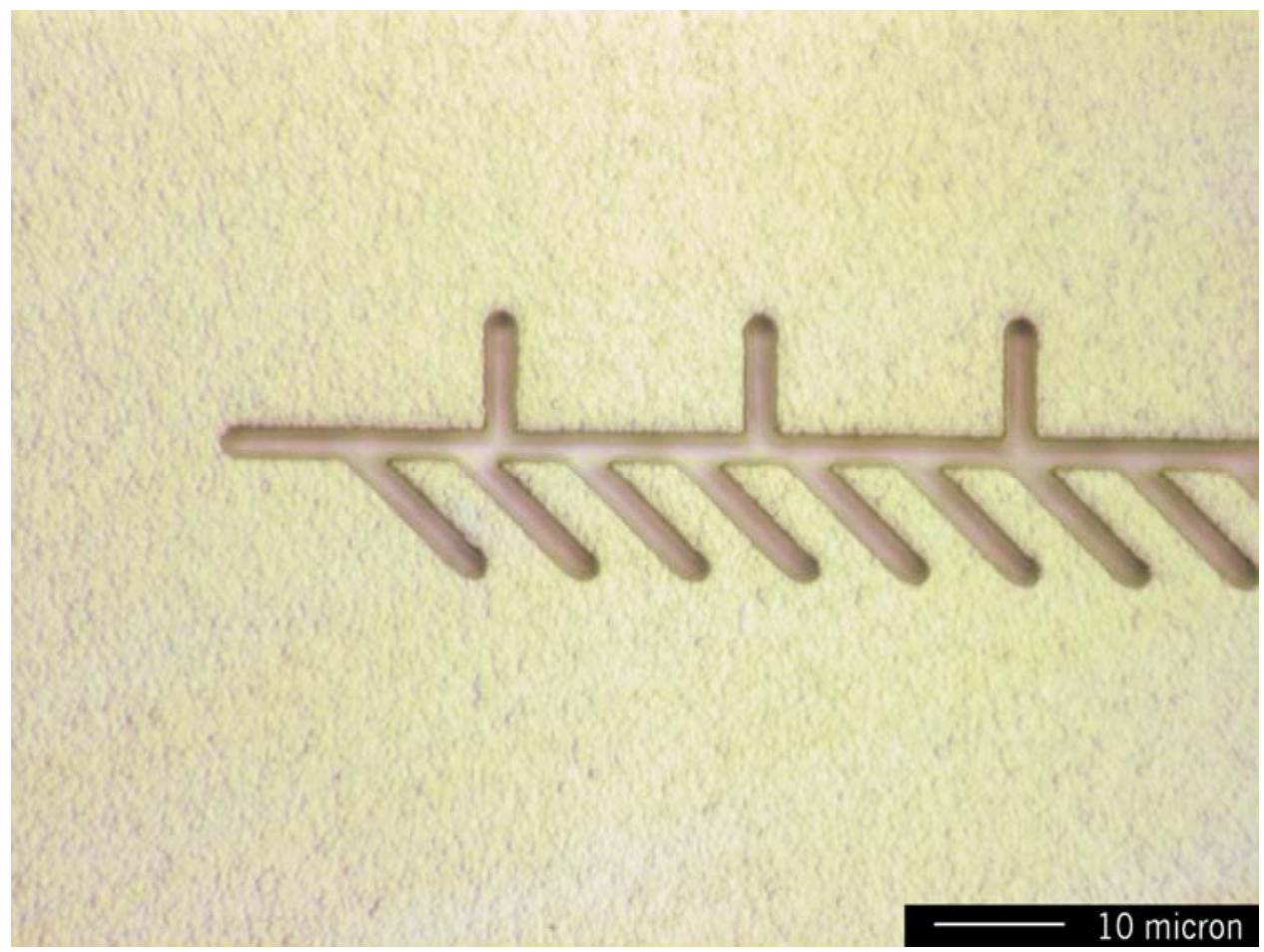

Figure 3.12 Top view of the trench area after etching the first structural layer.

Next, photoresist was filled in the trenches. From this point on, all of the processes should be conducted at low-temperature $\left(<150{ }^{\circ} \mathrm{C}\right)$. Then, $2 \mu \mathrm{m}$ thick silicon film was sputtered on the wafer. The thickness of the sputtered silicon film was $21347 \pm 366 \AA$. This second structural layer was etched using trial recipe- 3 and photomask 'even layer'. Figure 3.13 shows the wafer top view after this process. After filling photoresist, the third structural layer was sputtered. Its thickness was $22049 \pm 411 \AA$. For the third silicon layer, photomask 'odd layer' and trial recipe-3 were used to dig trenches in the silicon film as shown in Figure 3.14. 


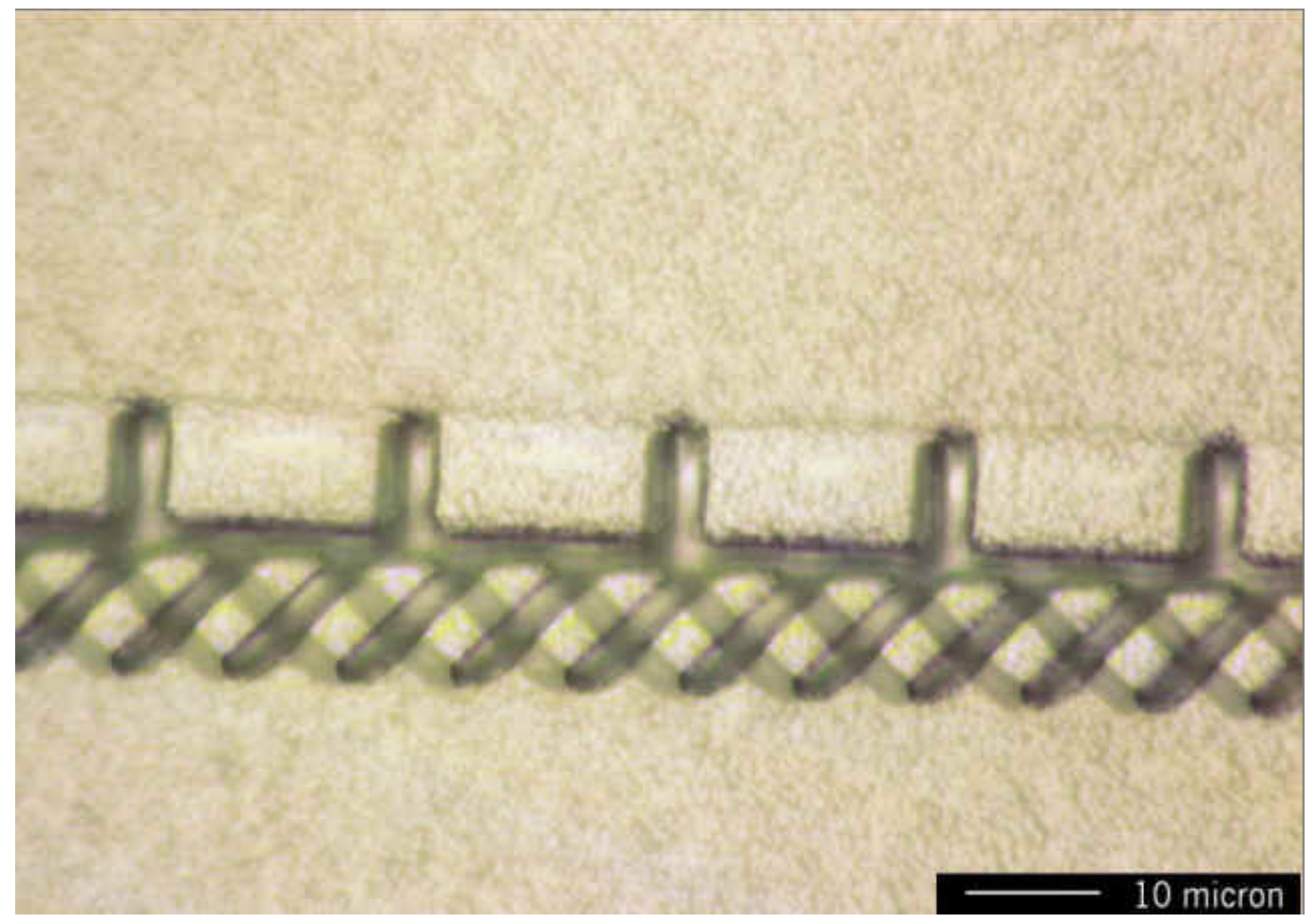

Figure 3.13 Top view of the trench area after etching the second structural layer

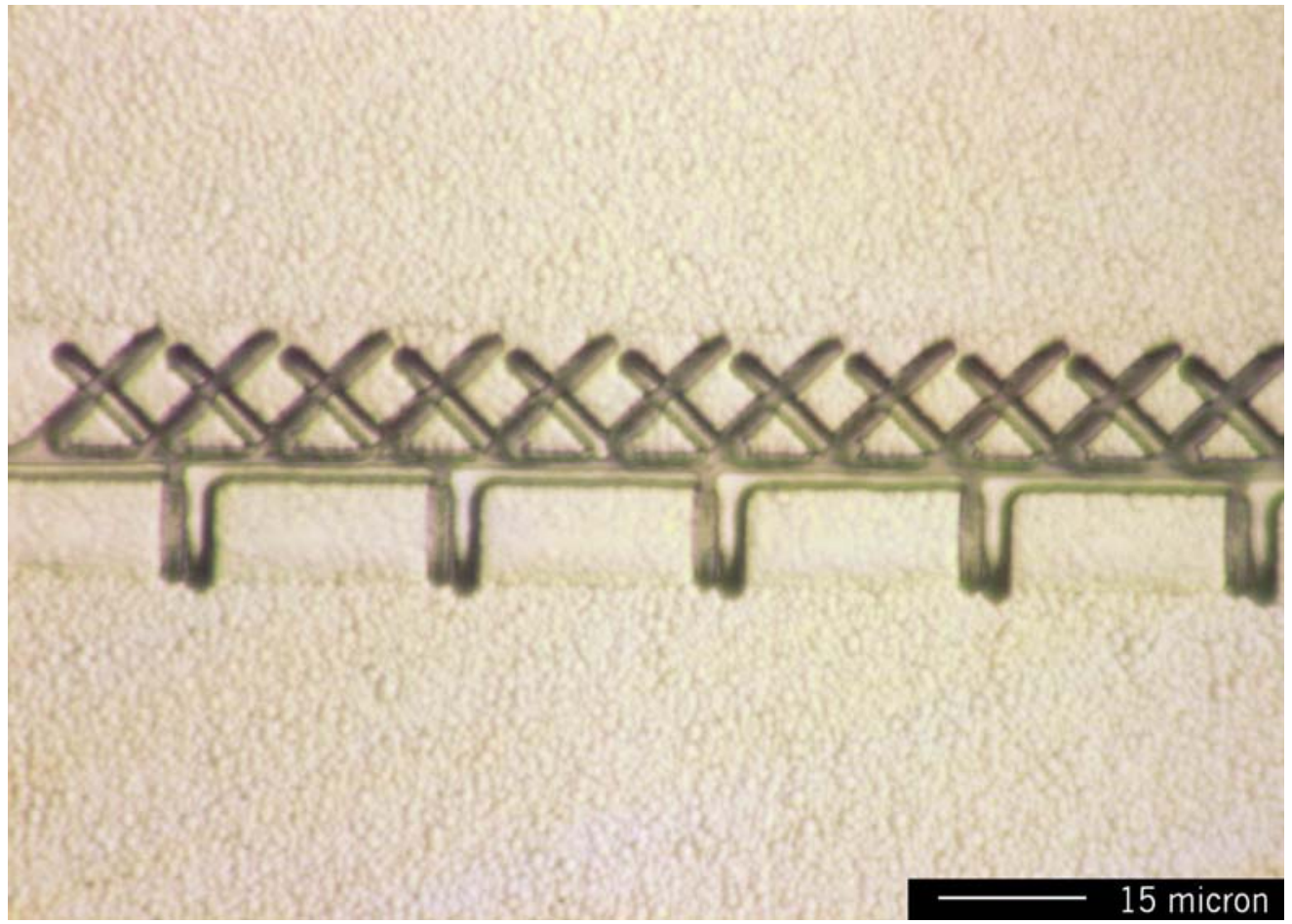

Figure 3.14 Top view of the trench area after etching the third structural layer. 
The last photoresist application process was done not only for filling the photoresist in the trenches but also for forming final etching mask. The photoresist film deposited in this step was patterned using photomask 'final structure'.

The total thickness of the three-layer silicon film was $6.8 \mu \mathrm{m}$. Based on the etch rate described in Appendix A.3, at least $2 \mu \mathrm{m}$ thick photoresist etching mask was needed. In order to ensure there was enough mask material to protect the fabricated microstructures, a $4 \mu \mathrm{m}$ thick photoresist film was applied using AZ9260 photoresist. Based on the curve in Figure A.12, the parameters shown in Table3.8 were used to apply the AZ9260 photoresist.

\begin{tabular}{|c|c|c|c|c|}
\hline & $\begin{array}{c}\text { Pre- photoresist } \\
\text { application }\end{array}$ & Pouring photoresist & Pre-spin & Spin \\
\hline Spin speed (rpm) & 2000 & 0 & 1000 & 6000 \\
\hline Time (second) & 30 & 0 & 15 & 60 \\
\hline
\end{tabular}

Table 3.8 Spin coater parameters for depositing 4 4 m thick AZ9260 photoresist.

The thickness of the AZ9260 photoresist film was measured after the developing process with photomask 'final structure.' It was represented by the step height over the microstructure, which was measured using Dek-tak. The measuring results are shown in Table 3.9.

\begin{tabular}{|c|c|c|c|c|c|}
\hline & M1 & M2 & M3 & M4 & M5 \\
\hline Thickness $(\AA)$ & 40333 & 41228 & 42026 & 41695 & 42151 \\
\hline Mean $(\AA)$ & \multicolumn{5}{|c|}{41497} \\
\hline
\end{tabular}

Table 3.9 Thickness of the AZ9260 photoresist film. 
Since the etching times in the studies of the trial recipe- 3 are very short, it is reasonable to ask if the etch rates of the silicon and the photoresist are still consistent after extensive etching.

In order to ensure a successful final etching, the etching process was divided into several steps with one minute etching in each step until the silicon films were completely etched away. An accurate method for determining if the silicon films were completely etched away was to measure the thickness of the polysilicon film on the oxide film using Nanospec4000. If the thickness was zero and oxide film was about $2 \mu \mathrm{m}$ with good curve fit, then the etching process was finished. The whole etching procedure and the step heights over the microstructures are shown in Table 3.10.

\begin{tabular}{|l|c|c|c|c|c|}
\hline & M1 & M2 & M3 & M4 & M5 \\
\hline Original step height $(\AA)$ & 40333 & 41228 & 42026 & 41695 & 42151 \\
\hline After 1 min etching $(\AA)$ & 55873 & 56861 & 55120 & 56724 & 60305 \\
\hline After 2 mins etching $(\AA)$ & 69166 & 70238 & 73152 & 71573 & 72152 \\
\hline After 3 mins etching $(\AA)$ & 85060 & 86841 & 86247 & 87613 & 88877 \\
\hline $\begin{array}{l}\text { Polysilicon thickness on the oxide } \\
\text { film after 3 mins etching }(\AA)\end{array}$ & 4029 & 2495 & 2069 & 2247 & 1154 \\
\hline $\begin{array}{l}\text { AZ9260 etch rate within the first 3 } \\
\text { mins }(\AA / \text { min) }\end{array}$ & 6572 & 6788 & 7394 & 6769 & 6864 \\
\hline After 4 mins etching $(\AA)$ & 82389 & 82448 & 81578 & 83587 & 83535 \\
\hline $\begin{array}{l}\text { AZ9260 etch rate within the first 4 } \\
\text { mins }(\AA / \text { min) }\end{array}$ & 6604 & 6813 & 7230 & 6645 & 6772 \\
\hline $\begin{array}{l}\text { Silicon etch rate within the first one } \\
\text { minute }(\AA / \text { min) }\end{array}$ & 22144 & 22446 & 20324 & 21674 & 24926 \\
\hline $\begin{array}{l}\text { Silicon etch rate within the second } \\
\text { one minute }(\AA / \text { min) }\end{array}$ & 19897 & 20190 & 25262 & 21494 & 18619 \\
\hline $\begin{array}{l}\text { Silicon etch rate within the third one } \\
\text { minute }(\AA / \text { min) }\end{array}$ & 22498 & 23416 & 20325 & 22685 & 23497 \\
\hline
\end{tabular}

Table 3.10 Final processes for etching three-layer silicon films with trial recipe-3. 
After one minute of etching, the area opposite to the main flat was etched faster than the area around the main flat with a difference of about $5000 \AA / \mathrm{min}$. Whether the difference was caused by different silicon etch rates or by different photoresist etch rates in the two areas was difficult to ascertain. After rotating the wafer by $180^{\circ}$, the wafer was etched for another one minute. This time, the step height difference was not very significant.

So far, the good curve fits were still not obtained when using Nanospec4000 to measure polysilicon thickness. This meant that the sputtered silicon had not been completely etched away. After etching the silicon film for the third one minute, polysilicon thickness with good curve fit was obtained. At this point, we can estimate the etch rate of the AZ9260 photoresist within the first three minutes by assuming the total silicon thickness is $68472 \AA$. The data shown in the seventh row of Table 3.10 were consistent with the data in Table A.18. Therefore, it was believed that the etch rate of the photoresist was consistent with trial recipe-3.

The wafer was loaded in the etching chamber for the fourth one minute etching so that the silicon film could be completely etched away under the over-etching condition. After this step, the etch rate of AZ9260 was calculated. The results are shown in the ninth row of Table 3.10. Thereafter, silicon etch rates within the one-minute step were calculated.

Generally, the silicon etch rate in this process is slower than that obtained in Appendix A.2.4, while the AZ9260 etch rate is a little faster than Shipley 1813. As shown in Column M3 and M5 of Table 3.10, the etch rate was much faster at the location of M5 than that at the location of M3 in the first one-minute. In the second one minute, 
M3 was faster than M5 because the wafer had been rotated by $180^{\circ}$. This etching problem may be caused by non-uniform distribution of the gases or other uncertain factors in the etching chamber.

After 4 minutes etching, the three-layer silicon microstructures were formed. A top view of the microstructure is shown in Figure 3.15. Thereafter, the wafer was put in BOE (Buffered oxide Etch) solution to etch the silicon dioxide film (sacrificial layer) so that the double-clamped beams could be released and suspended over the substrate.

First, a test wafer with $19932 \pm 7 \AA$ thick oxide film was dipped into BOE for 15 minutes and then the thickness was $9583 \pm 12 \AA$, and the corresponding etch rate was $690 \AA / \mathrm{min}$. Since the widest feature size on the wafer was $20 \mu \mathrm{m}$ and the releasing critical length was $10 \mu \mathrm{m}$, etching time should be $100000 \AA / 690(\AA / \mathrm{min})=145 \mathrm{mins}$.

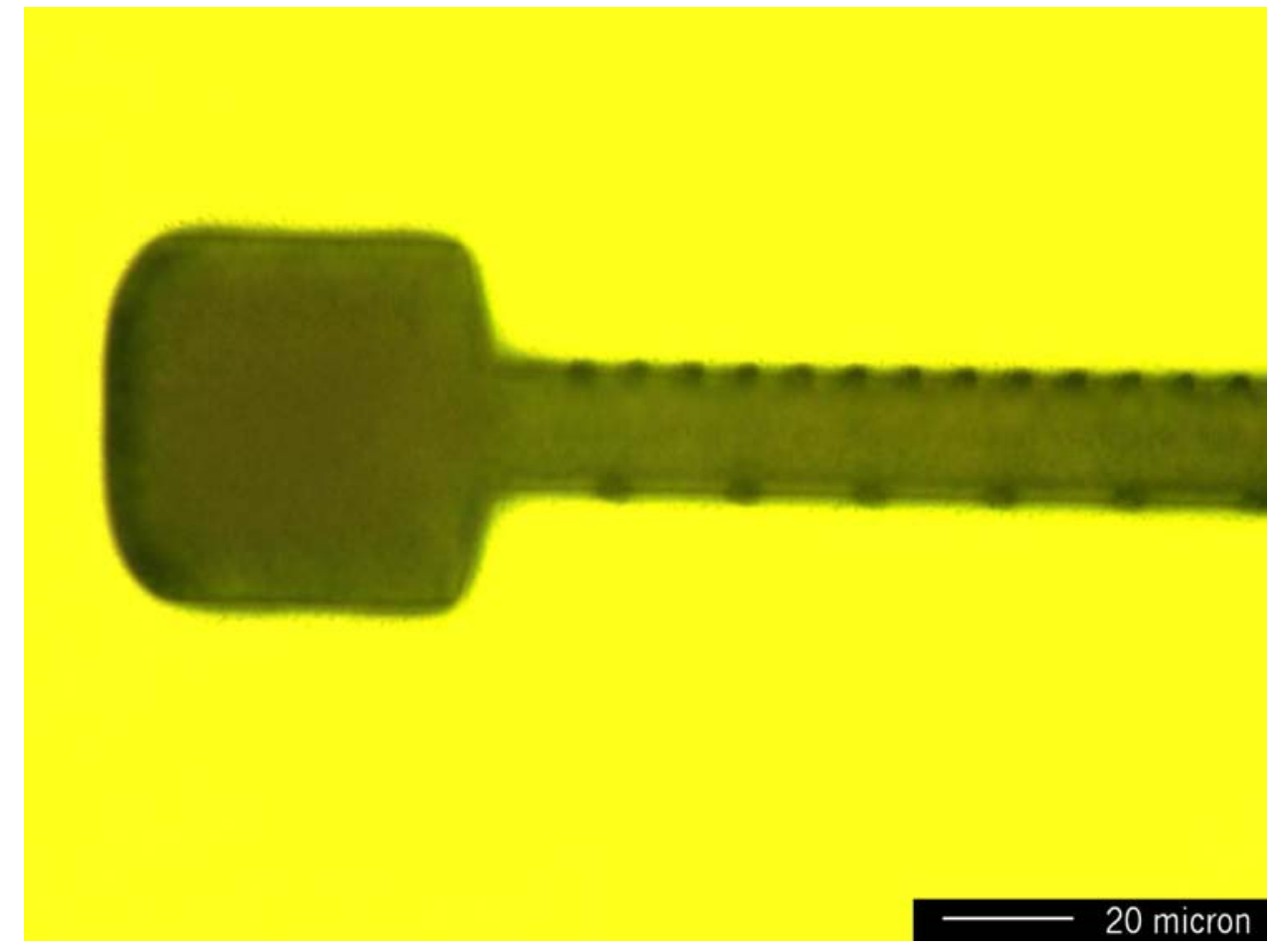

Figure 3.15 Top view of the three-layer silicon beam after being etched for 4min.using trial recipe-3 
The wafer with specimens was dipped in the BOE solution for 155 minutes to completely release the microstructures. Figure 3.16 shows the top view of the released structure. The releasing result was checked by using micro-probe to push the microstructure. The beams moved and eventually broke off, which meant the beams were completely released. Figure 3.17 shows one of the broken beams being pushed by the micro-probe. Underneath the broken beam, the oxide film surface is very clean and no residual materials scatter on the surface. Therefore, the releasing process is successful, which means that the whole fabrication is finished successfully.

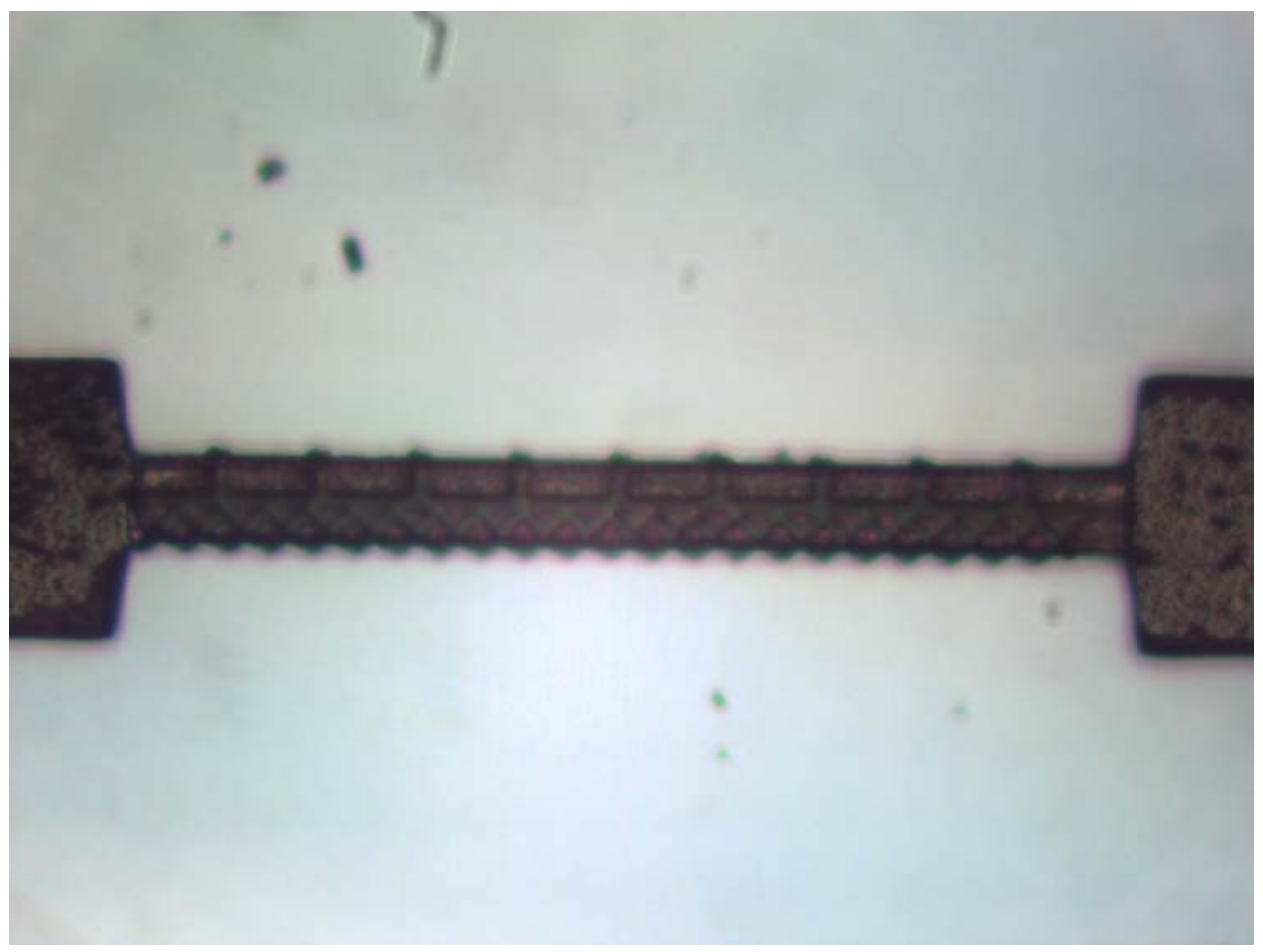

Figure 3.16 Top view of the three-layer microstructure after being released. 


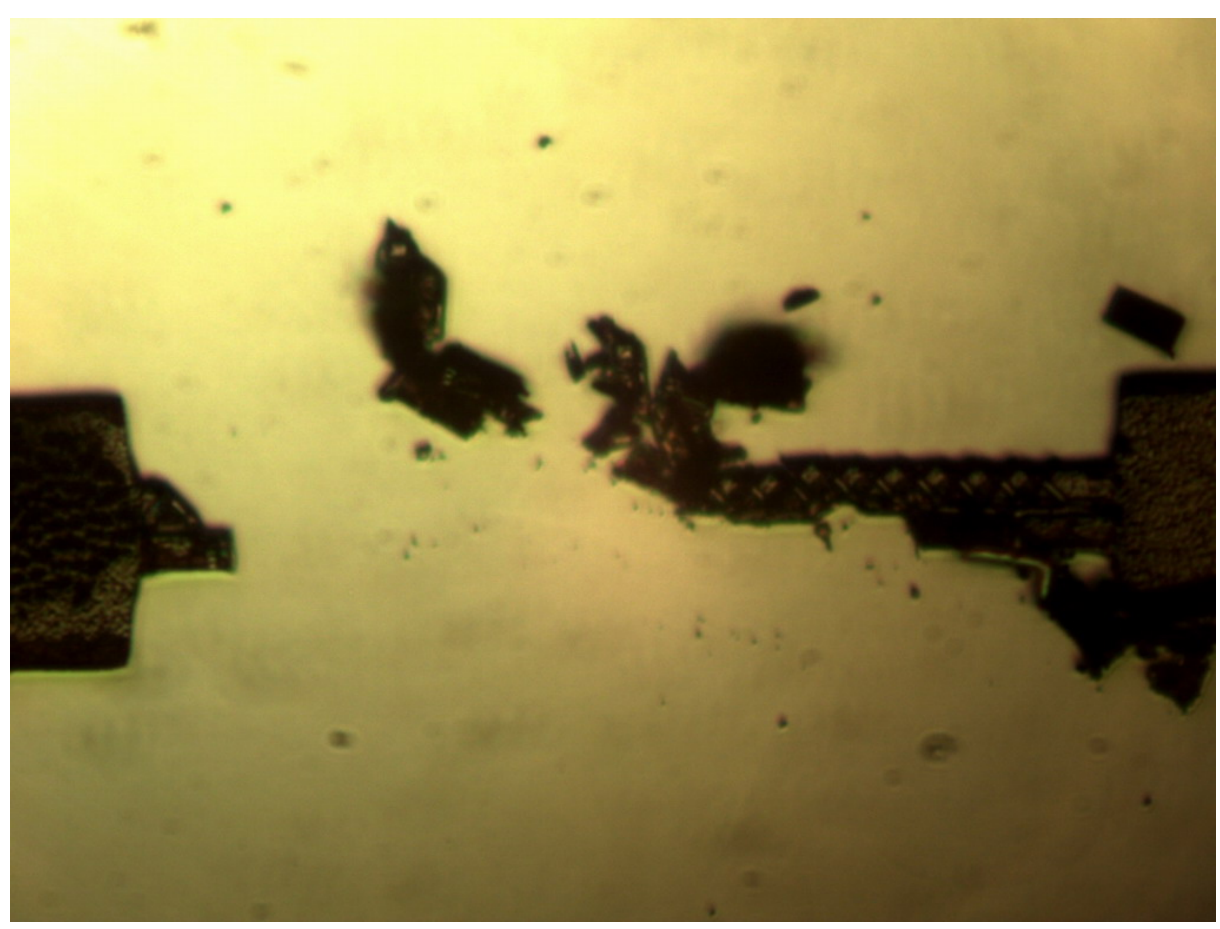

Figure 3.17 Top view of a broken microstructure. 


\section{Chapter Four}

\section{Nanoindenter and Material Modulus Testing}

\subsection{Nanoindenter}

A commercially available nanoindenter system (Triboscope, Hysitron, Inc.) was employed for conducting the microstructure testing. This system was mounted on a Nanoscope SPM system (Dimension 3100) manufactured by Veeco, Inc. Figure 4.1 and Figure 4.2 show the Triboscope controller and the SPM system stage.

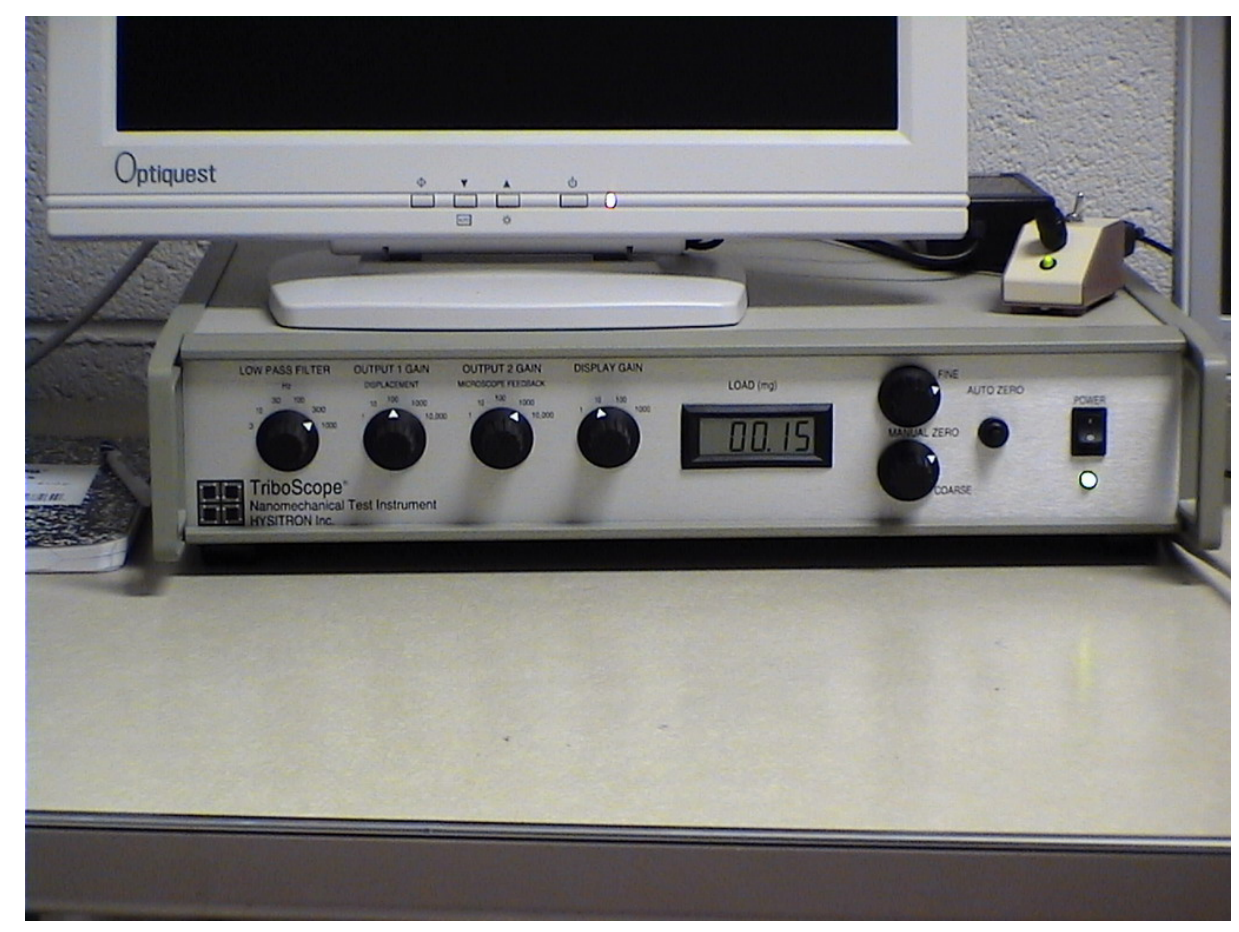

Figure 4.1 A Triboscope controller. 


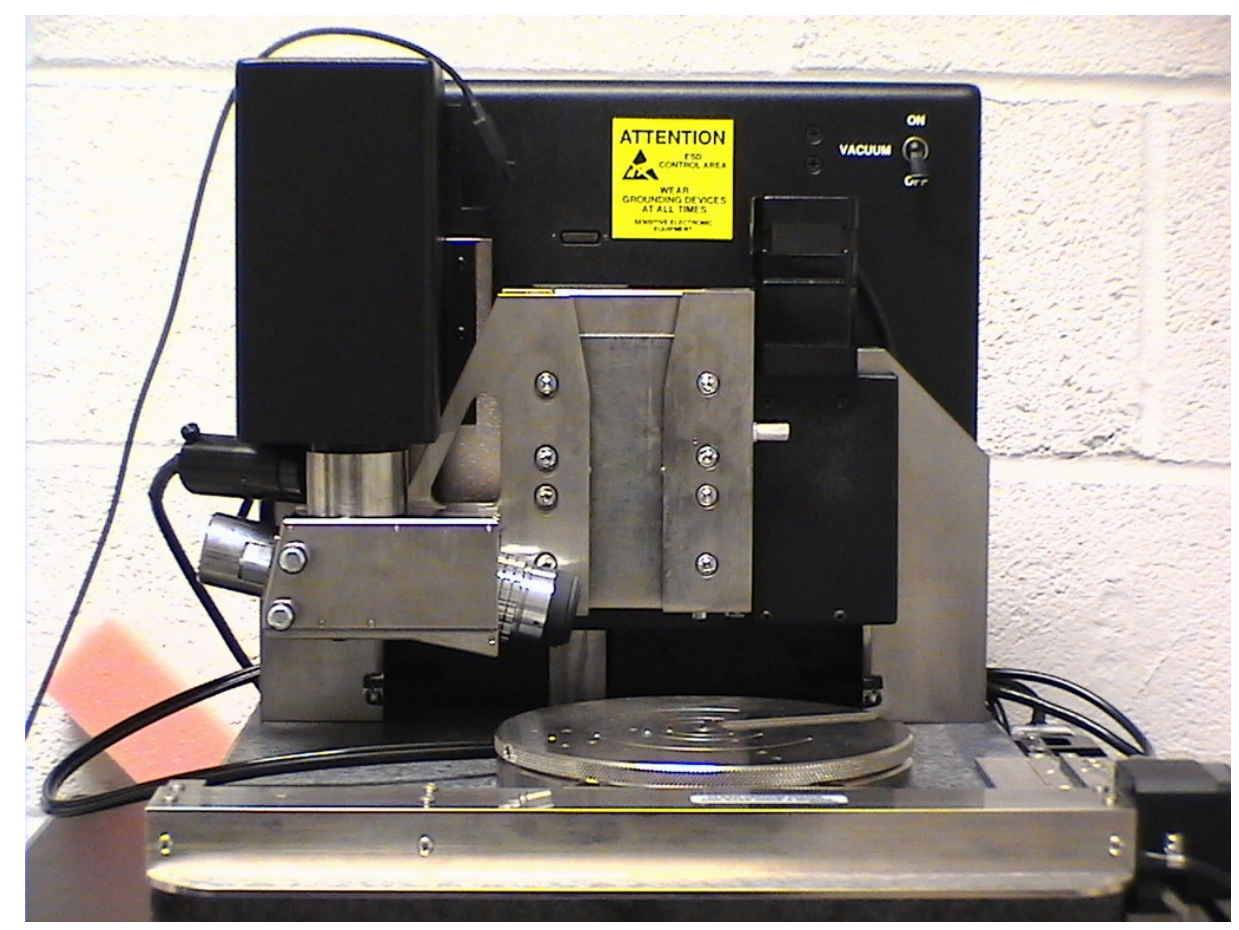

Figure 4.2 SPM system stage-Dimension 3100.

As shown in Figure 4.3 and Figure 4.4, two Hysitron transducers were employed for the tests: a 1-D transducer was used for indentation tests to determine the Young's modulus and hardness of the thin film materials; a 2-D transducer was used for applying lateral forces on the microstructures.

Compared to the 1-D transducer, the 2-D transducer has two additional forcedisplacement sensors to monitor and control the position of the indentation tip in the lateral direction. To set up the instrument, the indentation tip has to be mounted on the transducer. As shown in Figure 4.3 and Figure 4.4, the central hole with an internal screw bolt is the tip holder. The tip is screwed onto the bolt and connected to the spring system in the transducer. Figure 4.5 shows a tip mounted on a $2-\mathrm{D}$ transducer. 


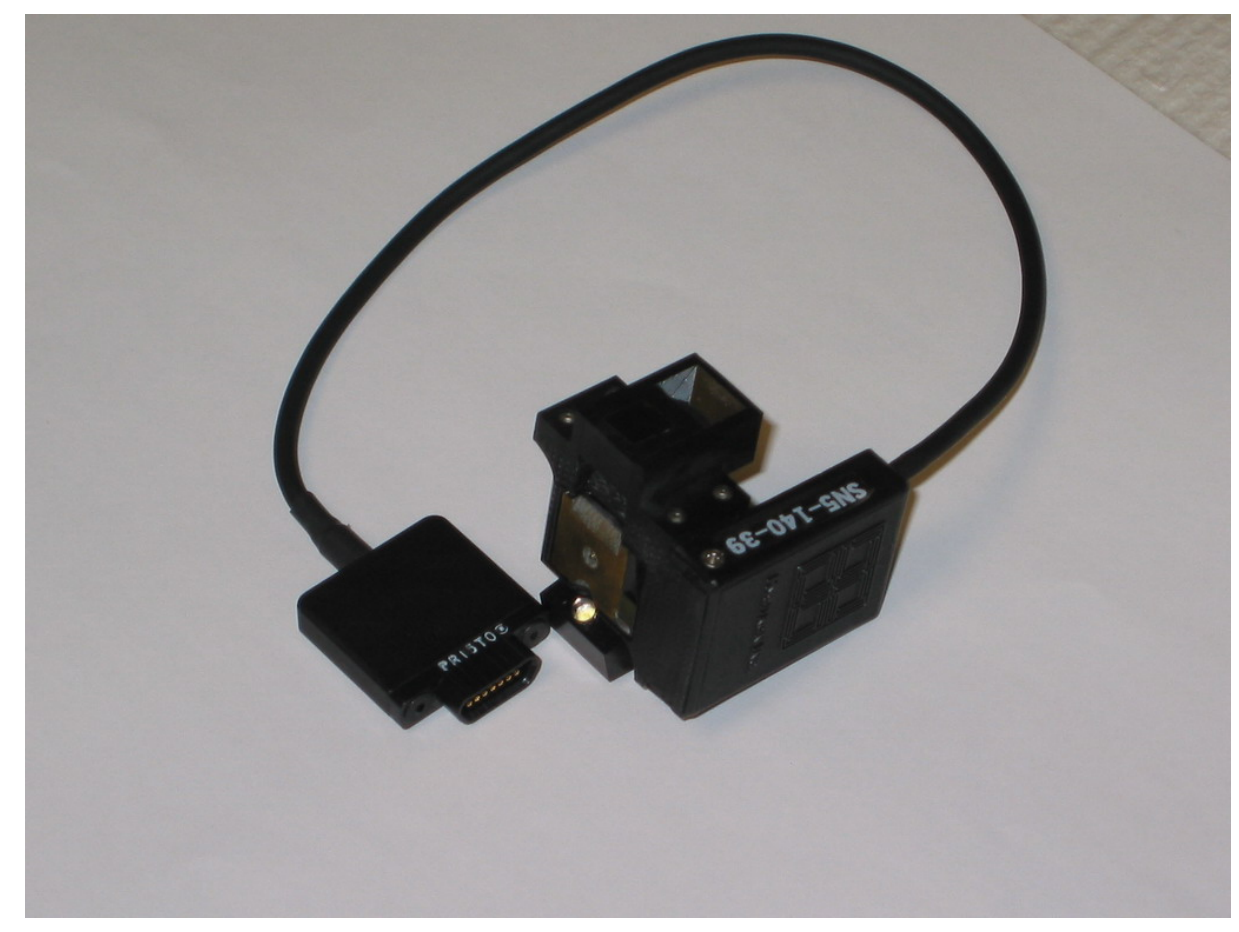

Figure 4.3 A 1-D transducer.

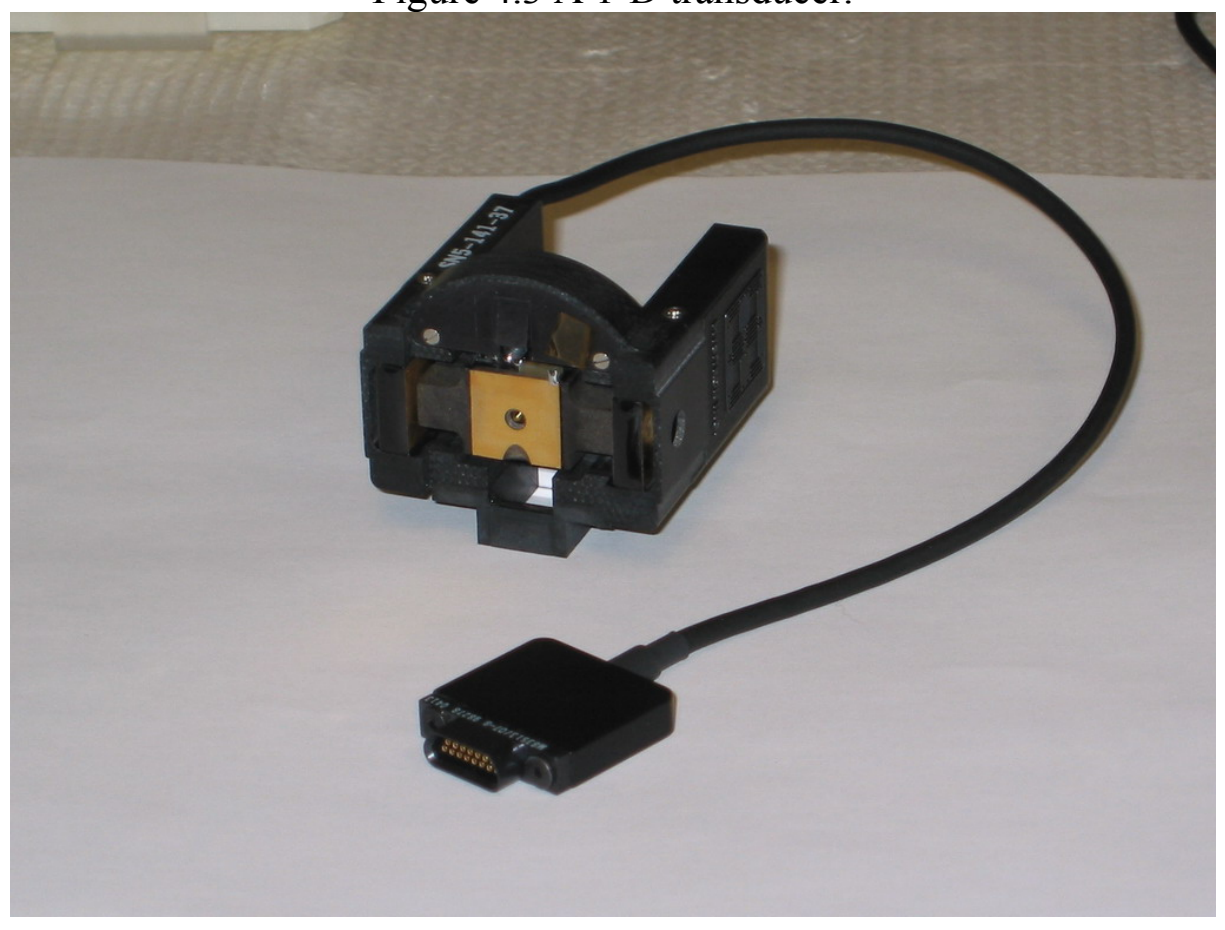

Figure 4.4 A 2-D transducer. 


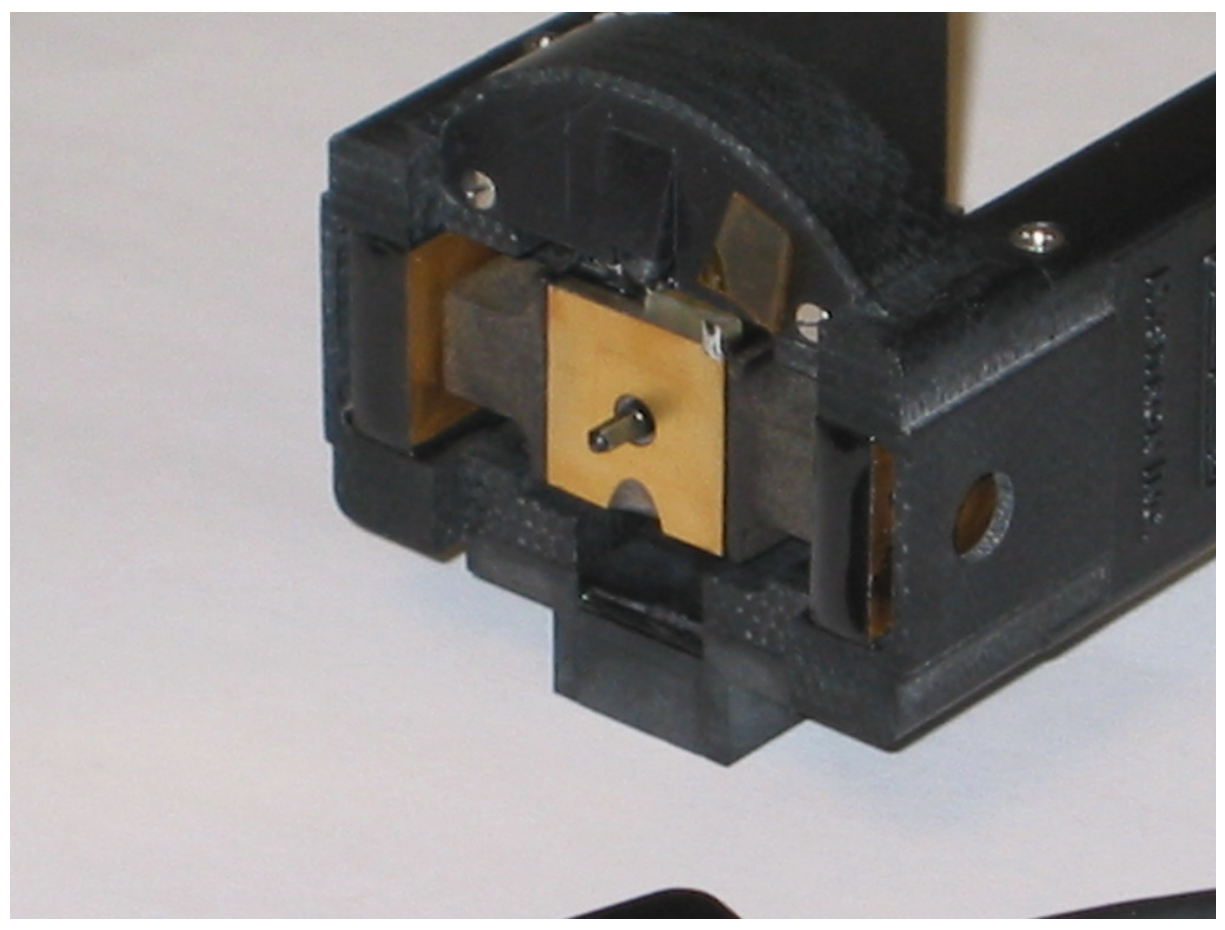

Figure 4.5 Indentation tip mounted on a 2-D transducer.

The transducer with indentation tip is assembled on a Triboscanner as shown in Figure 4.6. The Triboscanner attached with the transducer is slid into the slot in the vertical steel frame, and the signal cables are connected to the system controller as shown in Figure 4.7. The one on the right is the transducer cable connected to the Triboscanner, the one in the middle is the Triboscanner cable connected to the system controller and the one on the left is the power cable for the illumination system. With the help of the illumination system, the image of the tip can be transferred through the optical microscope to a built-in vision system and displayed in a vision window controlled by the Triboscope software. 


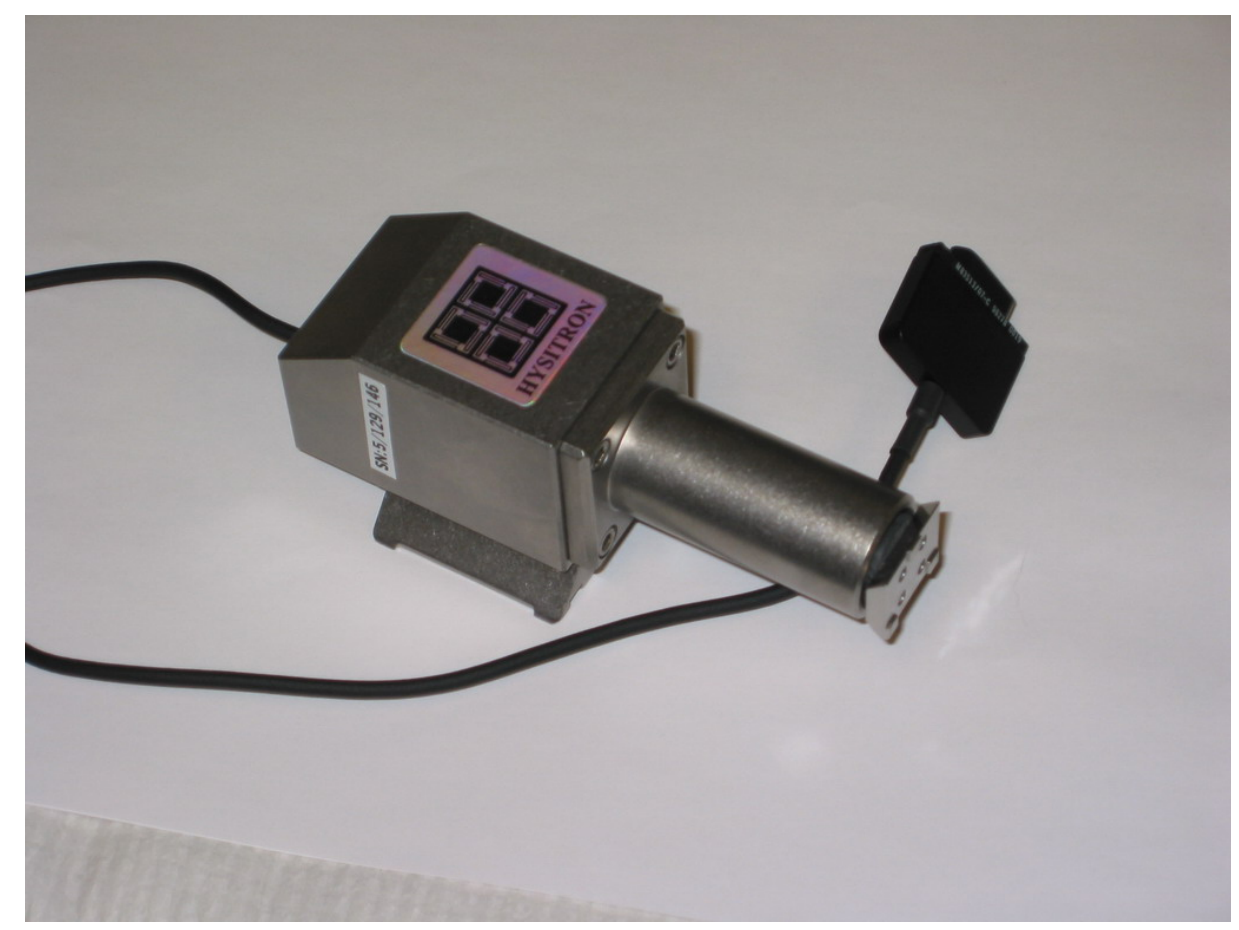

(a)

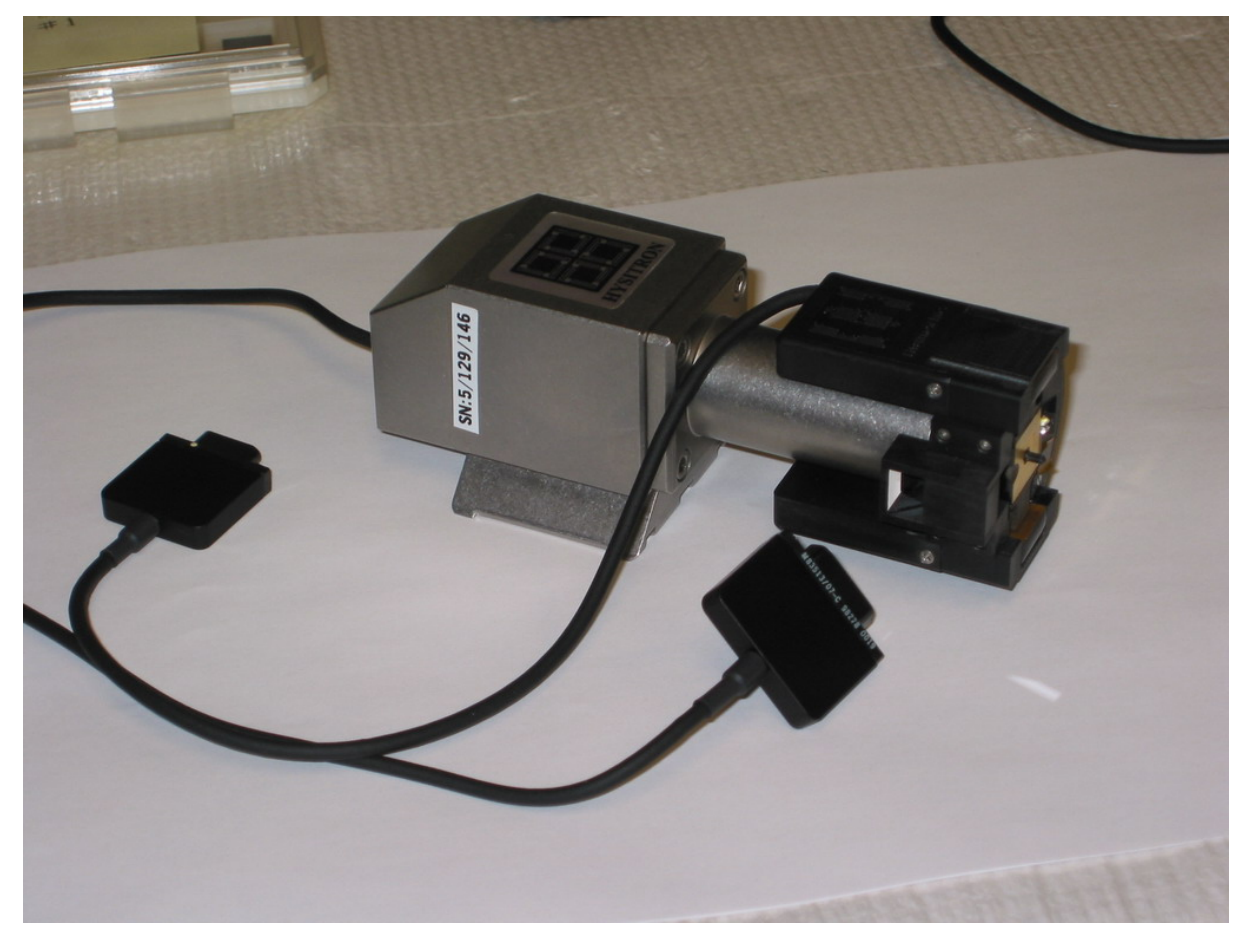

(b)

Figure 4.6 (a) Triboscanner; (b) Triboscanner with 2-D transducer. 


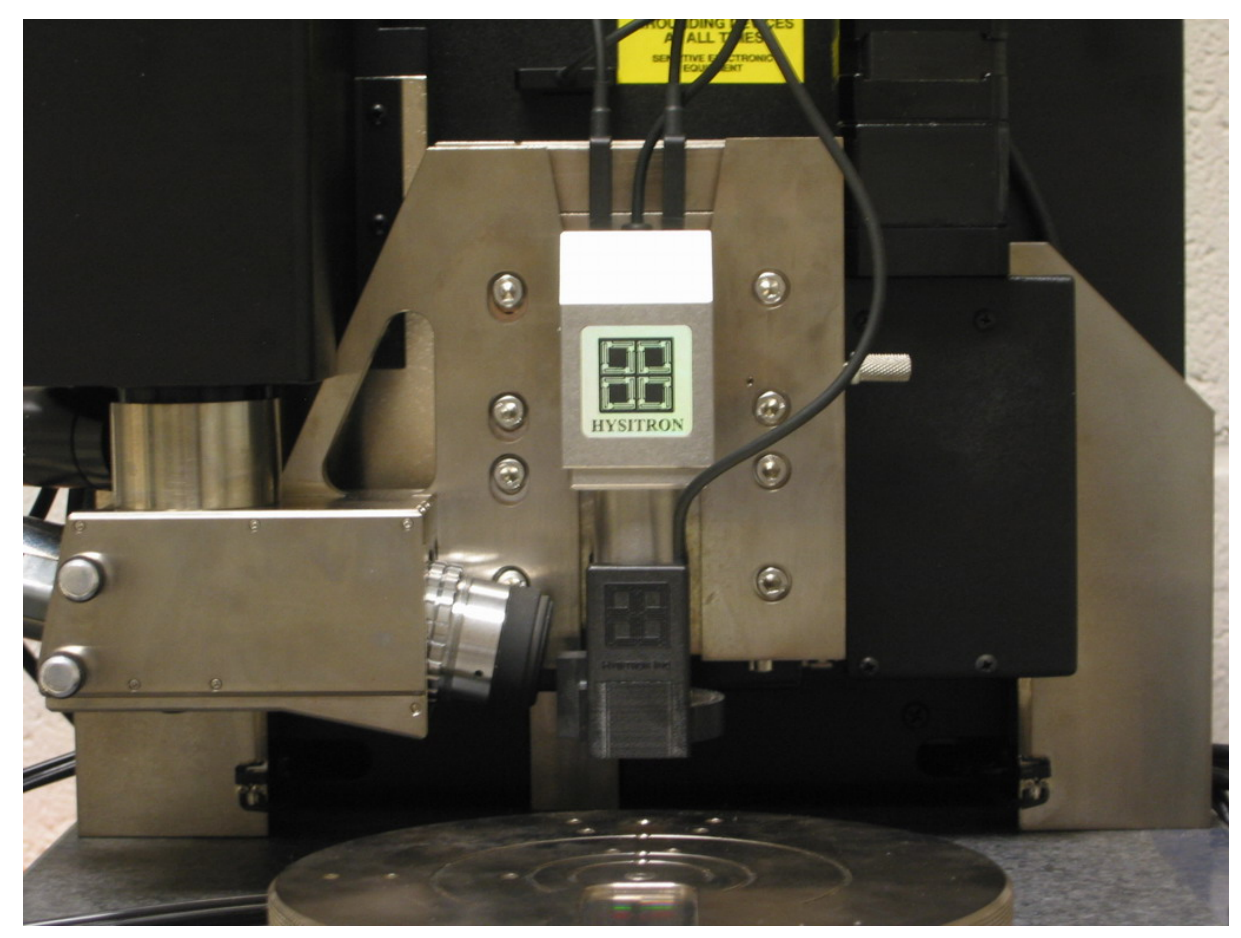

Figure 4.7 SPM stage with Nanoindentation transducer and tip.

The sample is fixed on the stage chuck by engaging the chuck vacuum system. A tracking ball is used to control the chuck X-Y position manually. Since the tracking ball cannot control the traveling length of the stage chuck, it has to be used with the vision system together to roughly locate the sample position. This will cause problems for accurately locating the specimens. This issue will be discussed in the following sections.

The 1-D transducer will be used for testing the Young's modulus for the sputtered silicon film and the baked photoresist film. The 2-D transducer will be employed to test the mechanical properties of the micro-specimens. 


\subsection{Measurement of the Elastic Modulus}

\subsubsection{Introduction of the indentation theory}

The theoretical foundation of the indentation method is the solution of the classic contact problem of an elastic half-space indented by a rigid punch. The key development for determining elastic modulus of thin film is the definition of the reduced modulus $\mathrm{E}_{\mathrm{r}}$ :

$$
\frac{1}{E_{r}}=\frac{1-v^{2}}{E}+\frac{1-v_{i}^{2}}{E_{i}}
$$

Where E and $v$ are the Young's modulus and Poisson's ratio of the sample and $\mathrm{E}_{\mathrm{i}}$ and $v_{\mathrm{i}}$ are the corresponding values of the indentation tip.

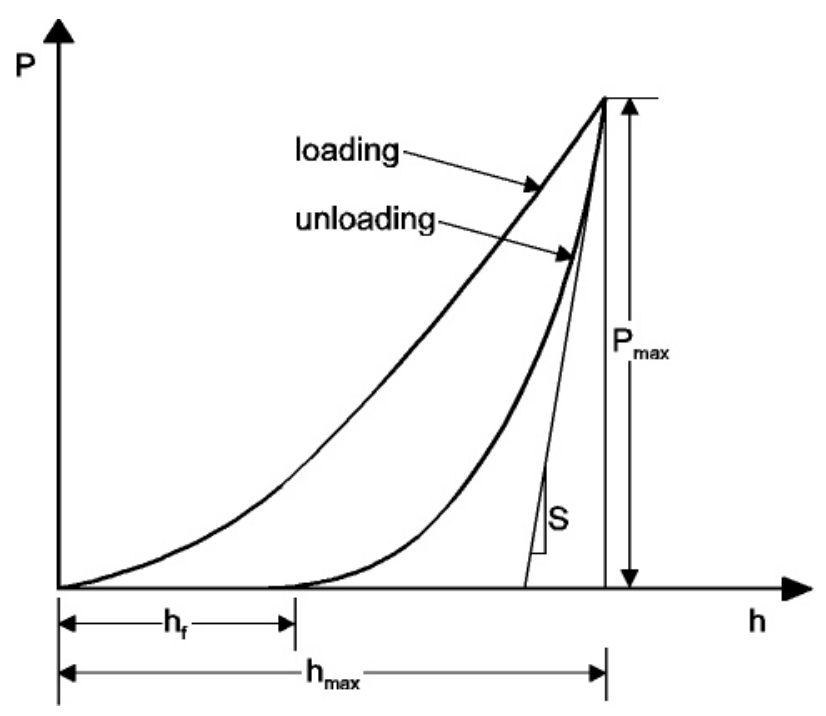

Figure 4.8 Load-displacement curve of the indentation.

The reduced modulus is determined by using load-depth curve of the indentation.

As shown in Figure 4.8, the displacement recovered during unloading is largely elastic and it can be described using the slope of the upper portion of the unloading curve as ${ }^{[24]-}$ [26]: 


$$
S=\frac{d P}{d h}=\frac{2}{\sqrt{\pi}} \beta E_{r} \sqrt{A}
$$

Where ' $\mathrm{S}$ ' is the initial unloading stiffness in the contact between the indentation tip and the sample, ' $A$ ' is the projected area of the elastic contact and $\beta$ is a parameter corresponding to the indentation tip shape.

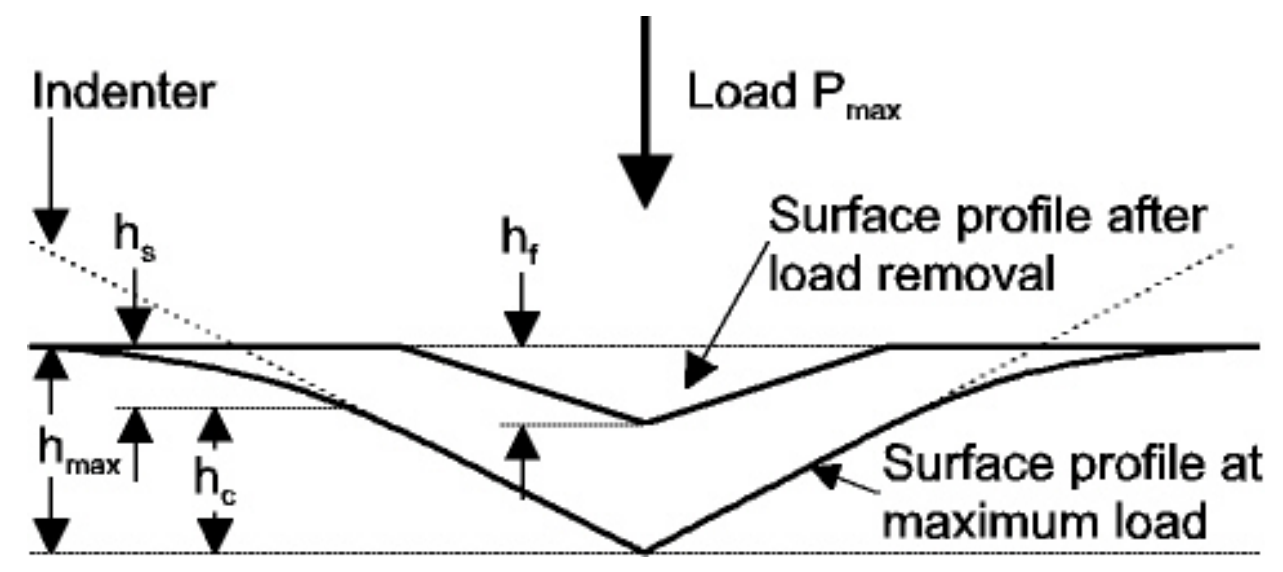

Figure 4.9 Schematic representation of a cross-section of an indentation.

Figure 4.9 depicts a cross-section of an indentation. Here, $\mathrm{h}_{\mathrm{c}}$ represents the vertical distance along which the contact is made; $\mathrm{h}_{\mathrm{f}}$ is the residual depth of the hardness impression after the unloading; $h_{s}$ is the displacement of the surface at the contact perimeter. The Berkovich indentation tip is considered as an example. Because the halfangle of the Berkovich indentation tip is $65.35^{\circ}$, the first estimate of the contact area is given by:

$$
A\left(h_{c}\right)=3 \sqrt{3} h_{c}^{2} \tan ^{2} \theta=24.5 h_{c}^{2}
$$

The real area function in terms of $h_{c}$ is determined by fitting the data of 'A' vs. ' $h_{c}$ ' to a nine-term polynomial ${ }^{[26]}$. Once the area function is obtained, the contact area 
can be calculated from the load-displacement data. Thereafter, the reduced elastic modulus and hardness can be computed as:

$$
\begin{aligned}
& E_{r}=\frac{1}{\beta} \frac{\sqrt{\pi}}{2} \frac{S}{\sqrt{A}} \\
& H=\frac{P^{\max }}{A}
\end{aligned}
$$

\subsubsection{Measurement of the Elastic modulus}

With Triboscope Nanoindentation system, the reduced Young's modulus and hardness are automatically calculated by the Triboscope system software. The actual procedure for fitting the load-displacement data and doing the calculation with Triboscope system is as follow:

1. Fit the selected portion of the unloading curve to the power law relation

$$
P=A\left(h-h_{f}\right)^{m}
$$

2. Evaluate the derivative of the power law with respect to $\mathrm{h}$ at the maximum load for calculating the contact stiffness S.

3. Calculate the contact depth $\mathrm{h}_{\mathrm{c}}$ using $h_{c}=h_{\max }-0.75 \frac{P_{\max }}{S}$

4. Calculate hardness using $H=\frac{P_{\max }}{A\left(h_{c}\right)}$

5. Calculate the reduced modulus using $E_{r}=\frac{\sqrt{\pi}}{2 \sqrt{A\left(h_{c}\right)}} S$ 
The Berkovich indentation tip was used for elastic modulus tests of the thin films. Combined with the SPM system, the Berkovich indentation tip can also function as a probe to scan the surface of the thin film and simultaneously depict the surface image before and after the indentation test. The Berkovich indentation tip used in this test is shown in Figure 4.10.

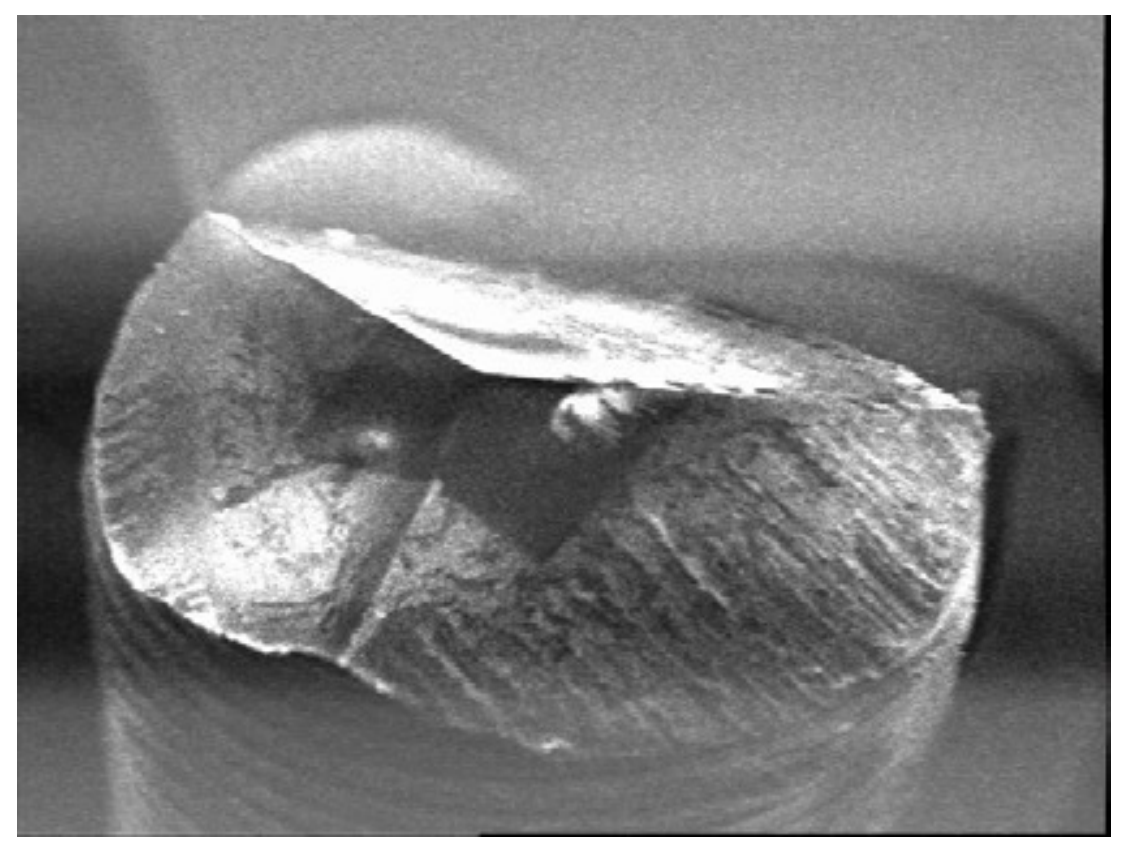

Figure 4.10 Berkovich indentation tip.

The area function of the Berkovich indentation tip was calibrated before the elastic modulus tests. This calibration was conducted to determine the coefficients of the polynomial by making more than ten indentations on the standard fused quartz and fitting these data to the area function. The Triboscope system software used six-term polynomial to describe the area function. Figure 4.11 shows the tip area function used for the indentation tests. 


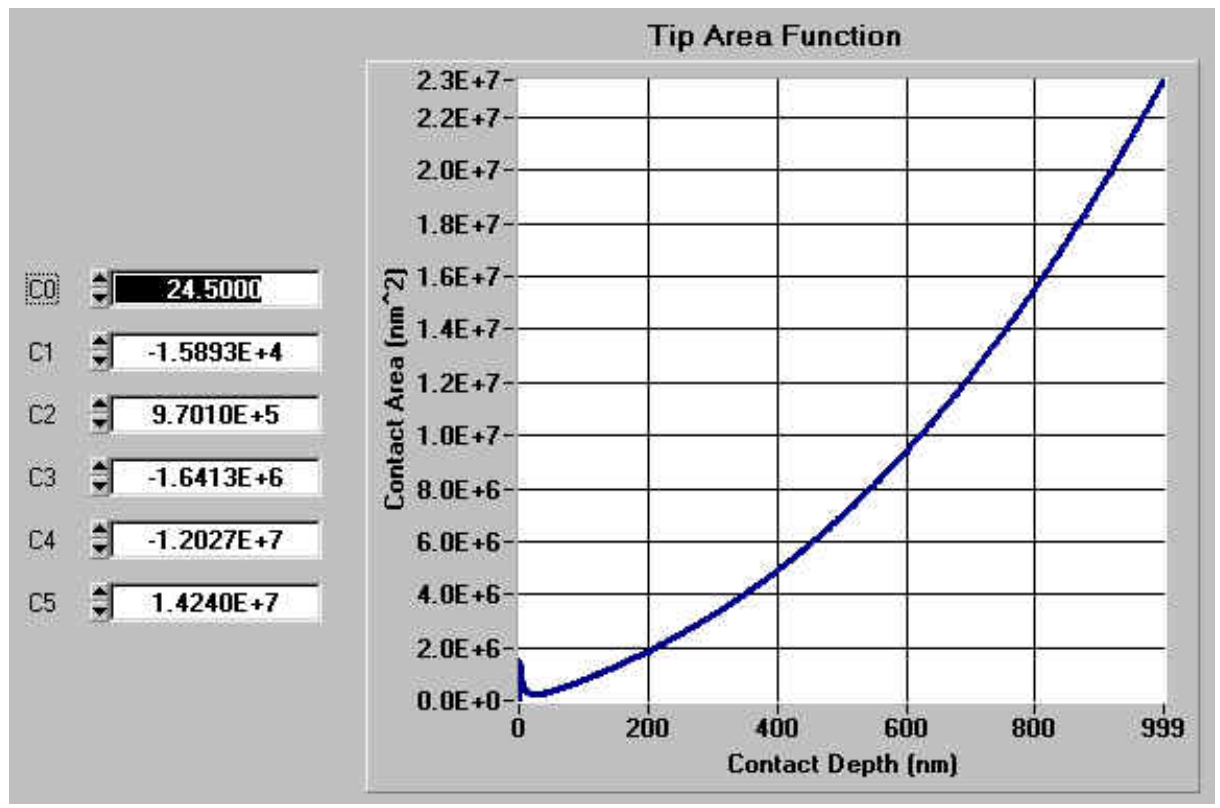

Figure 4.11 Area function of the Berkovich indentation tip.

After mounting the Berkovich indentation tip on the 1-D transducer and attaching the transducer to the system stage, the indentation system was calibrated in z-axis to ensure correct data collection. Thereafter, the specimen was mounted on the sample stage and the indentation tip was located right above the ideal indentation point. The indentation tip was engaged to contact the sample surface. Once it was engaged, the scan size was set to define the monitoring area. Typically, $5 \mu \mathrm{m}$ was used in the tests. Before the indentation test was triggered, the monitoring area was scanned to make sure no defects existed.

The silicon wafer was thermally oxidized to grow a $2 \mu \mathrm{m}$ thick silicon dioxide film. The sputtered silicon film was directly deposited on the oxide layer with a target thickness of $2 \mu \mathrm{m}$. Then, the wafer was diced into $0.8 \mathrm{~cm} x 0.8 \mathrm{~cm}$ chips. These chips were the basic testing units. 
The test was set to run with load control. A typical load function is shown in Figure 4.12. Three peak loads were used to detect the change of the reduced modulus versus the load levels to ensure appropriate load was used for collecting a meaningful reduced modulus.

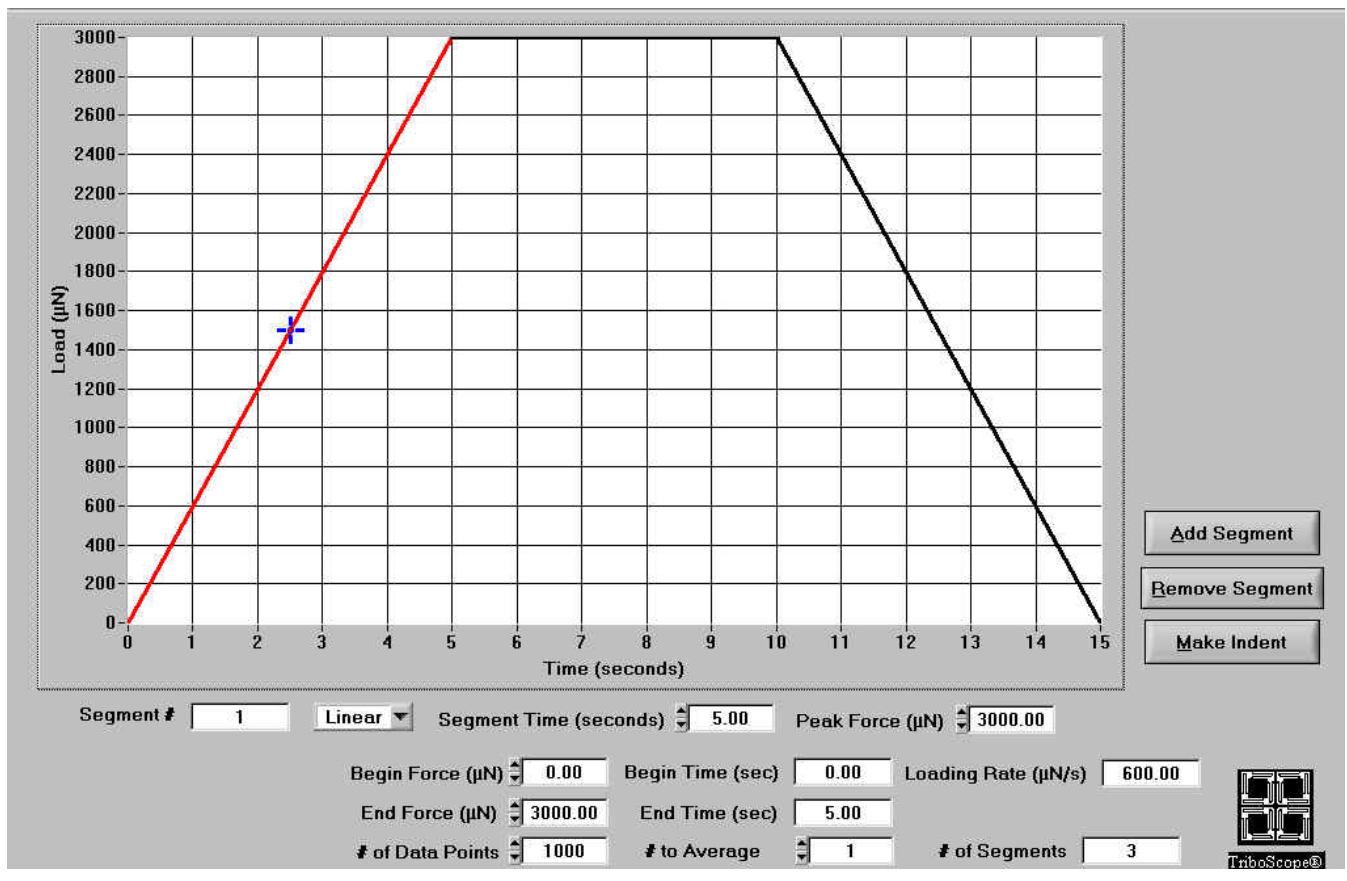

Figure 4.12 A typical load function for the indentation test.

The peak indentation load should lie in a certain region to ensure the testing results are accurate. The lower indentation limit is governed by the area function of the indentation tip. As long as the area function can generate accurate reduced modulus and hardness for the standard fused quartz, the corresponding indentation depth is appropriate for obtaining meaningful reduced modulus. The higher limit is the load needed to generate indentation depth greater than ten percent of the film thickness. On this indentation depth level, a variety of effects coming from the substrate begin to influence the testing accuracy. 
In order to find appropriate load level, three trial loads were set to make three indentation tests on the standard fused quartz and the sputtered silicon film. The three loads were $3000 \mu \mathrm{N}, 6000 \mu \mathrm{N}$ and $9000 \mu \mathrm{N}$. The load-displacement curves along with the reduced modulus and the hardness are shown in Figure 4.13 and Figure 4.14.

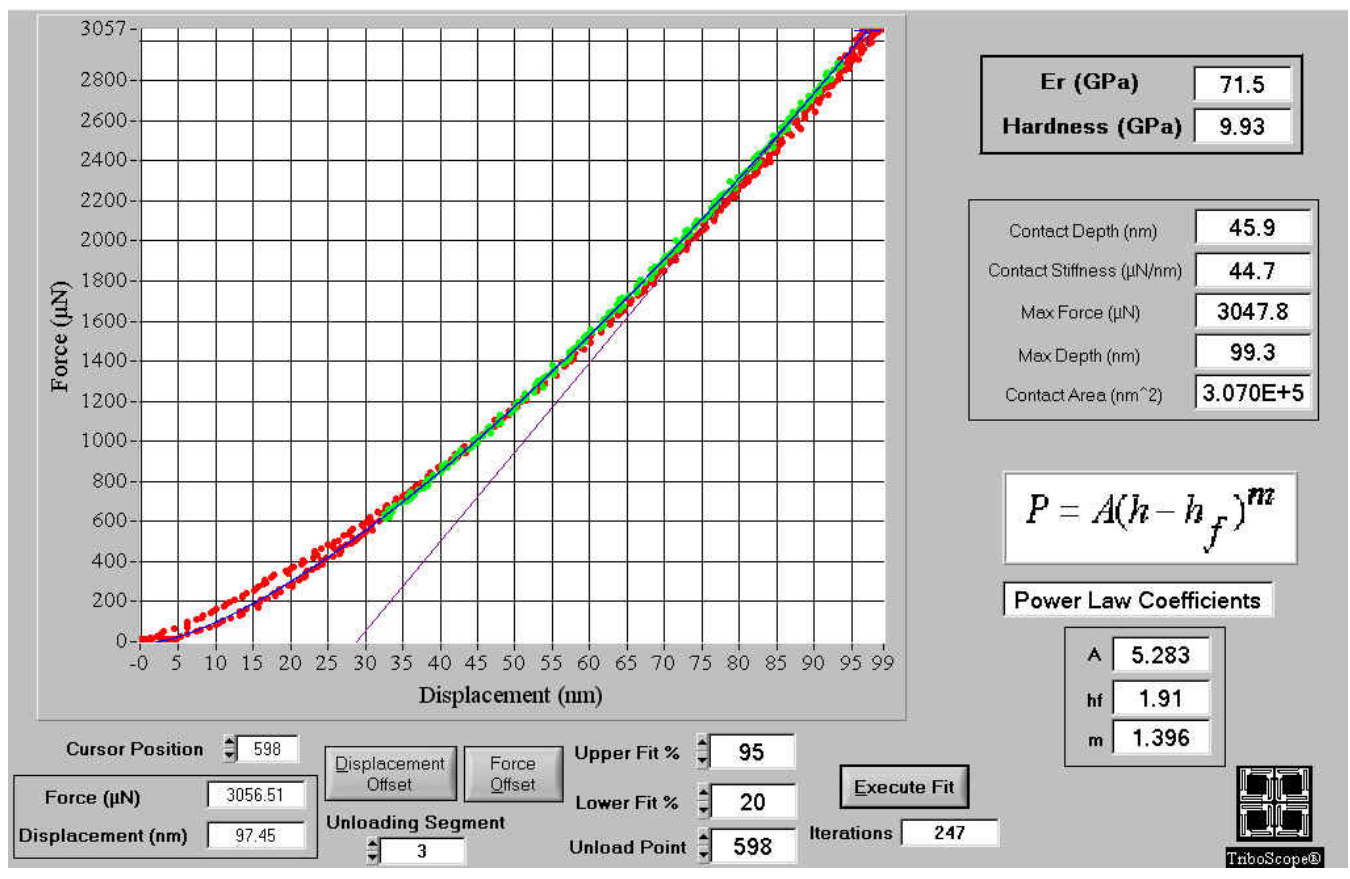

(a) 


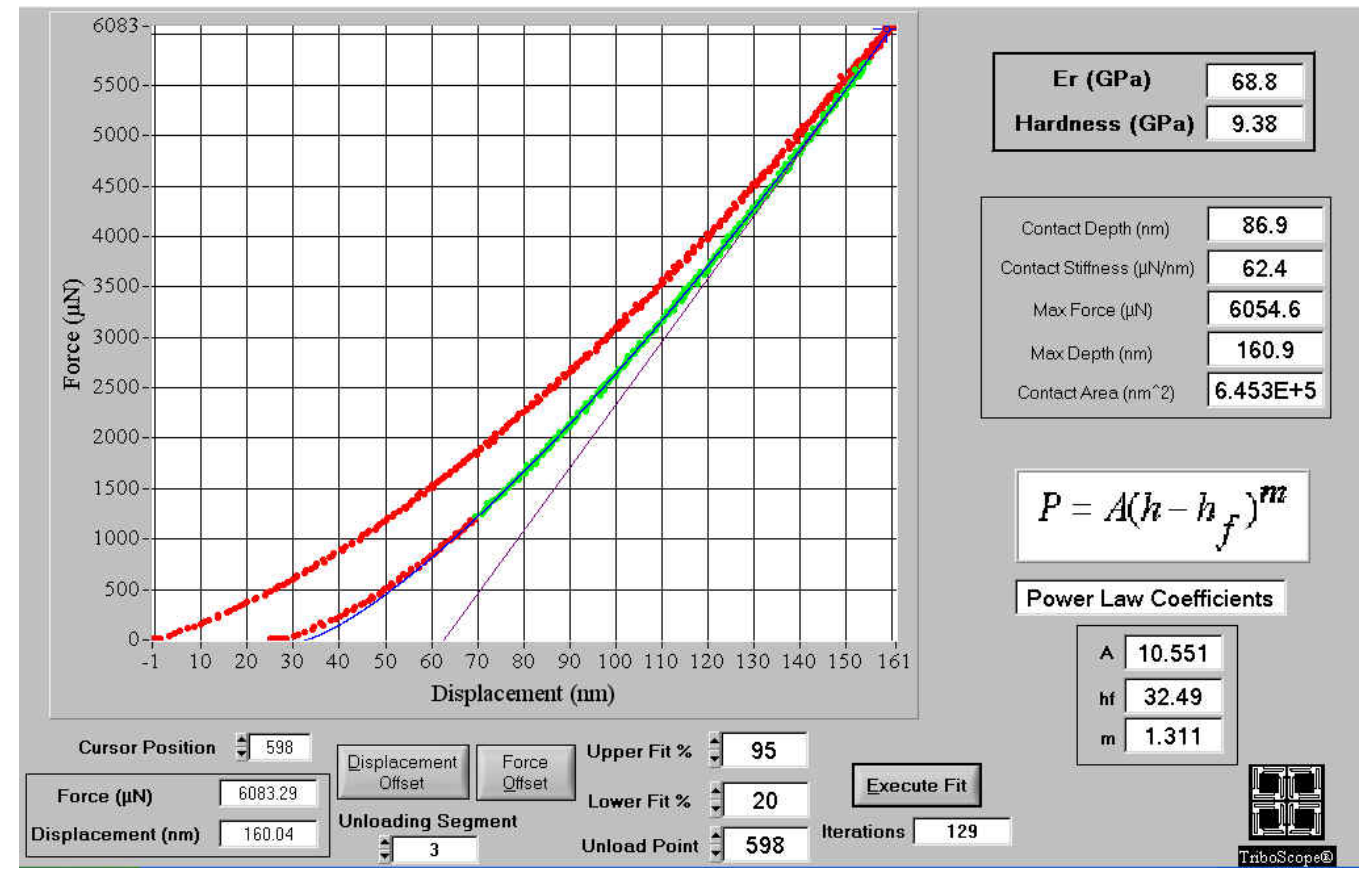

(b)

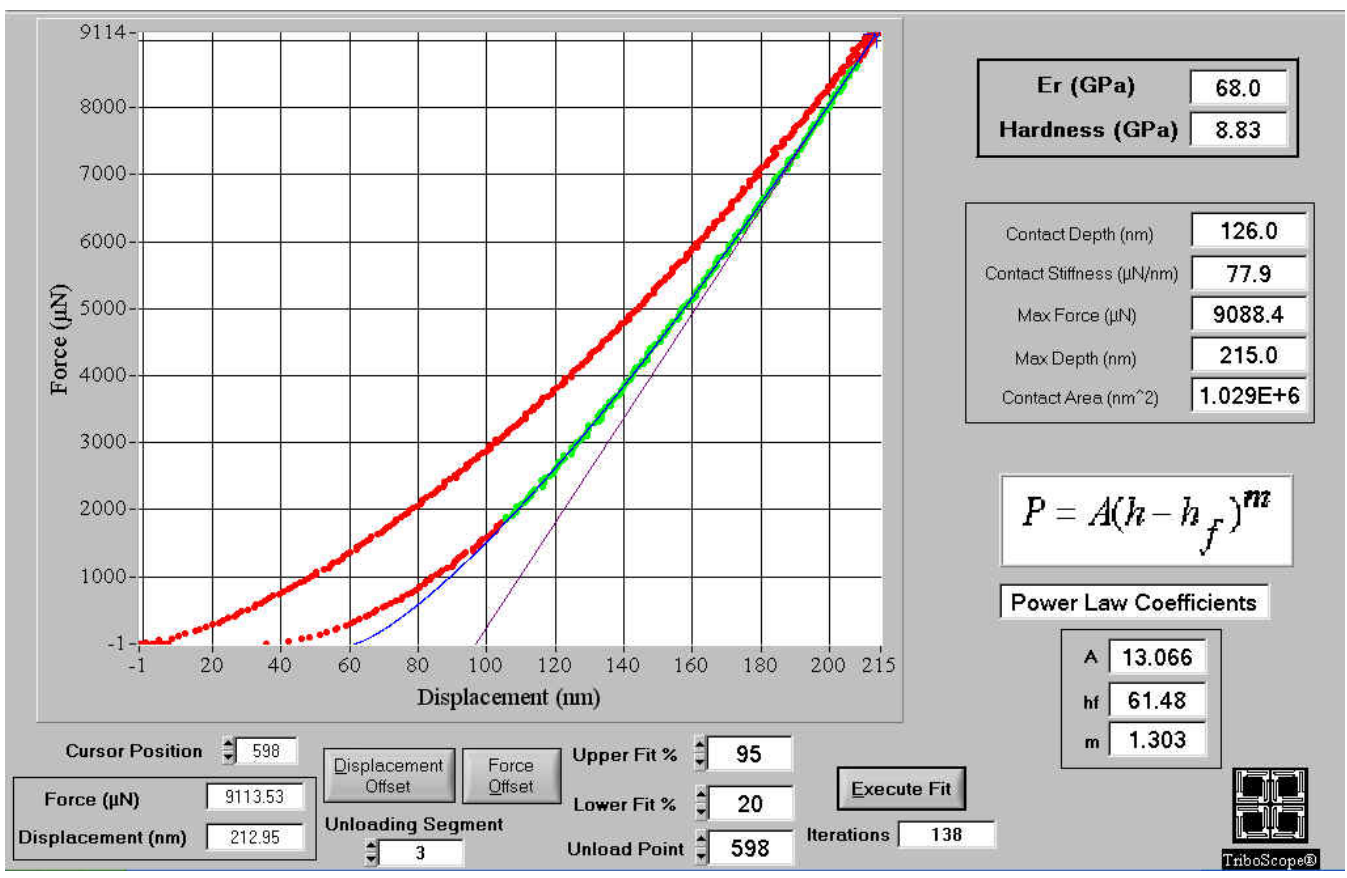

(c)

Figure 4.13 Testing results for standard fused quartz. (a) $3000 \mu \mathrm{N}$; (b) $6000 \mu \mathrm{N}$; (c) $9000 \mu \mathrm{N}$. 


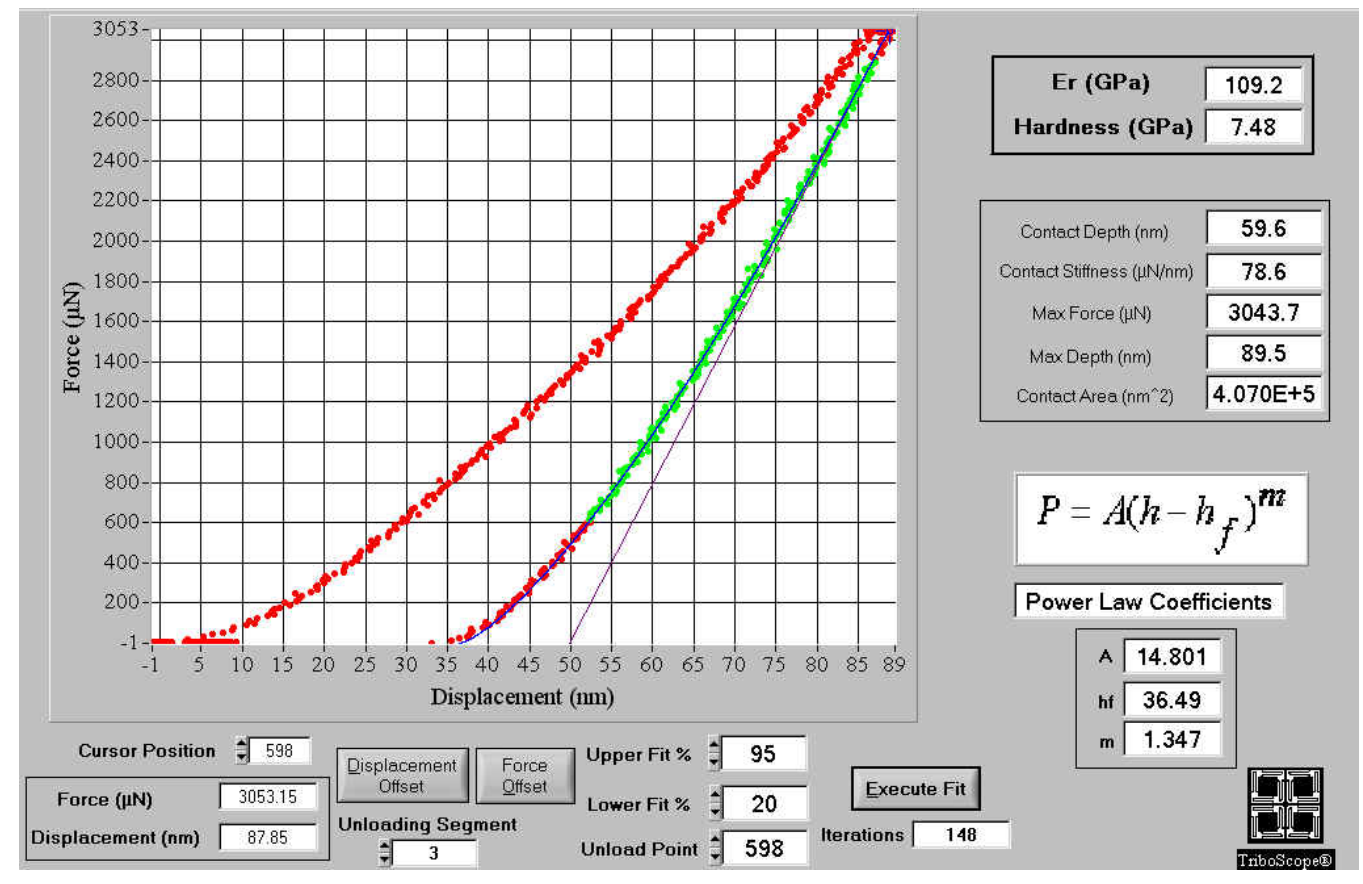

(a)

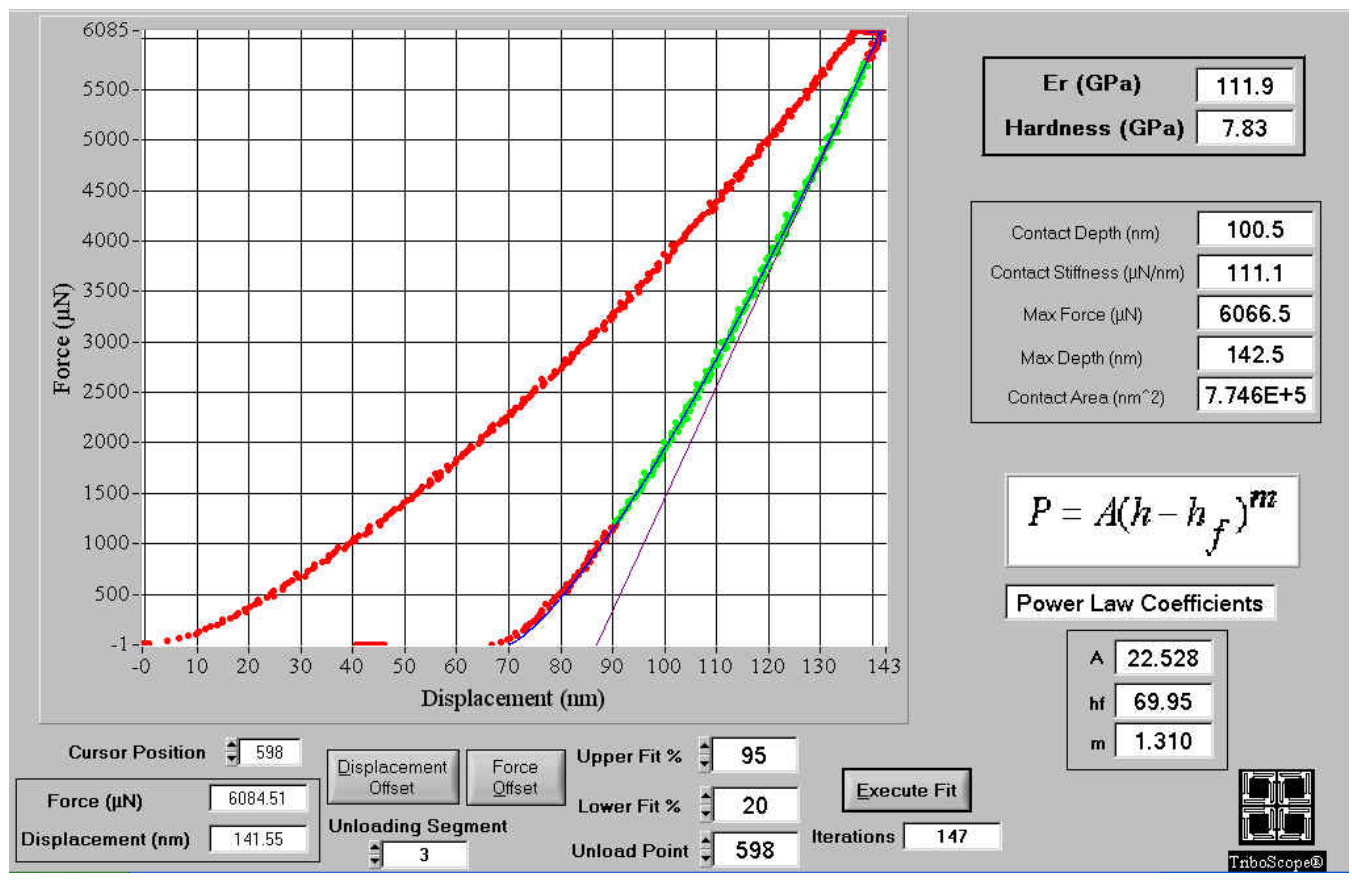

(b) 


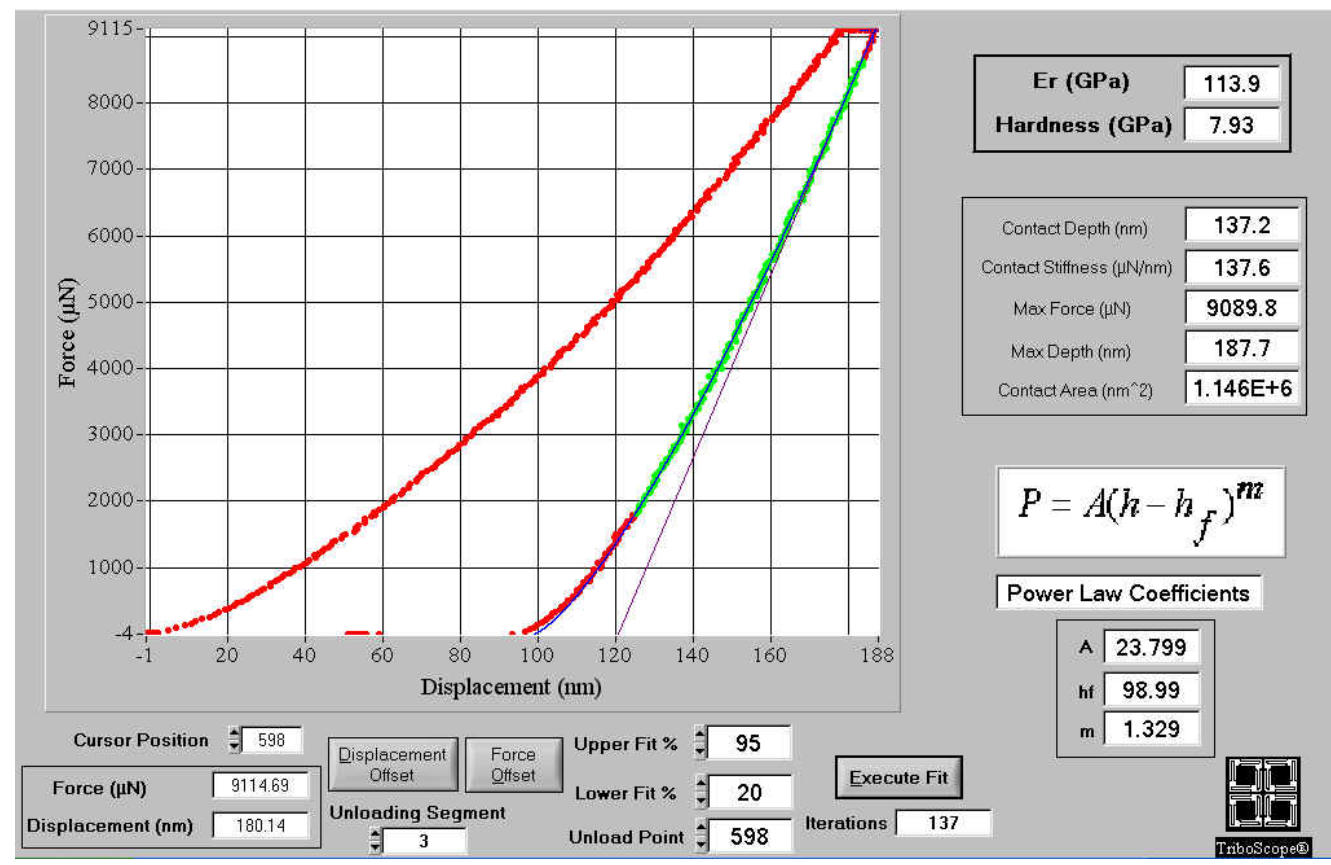

(c)

Figure 4.14 Testing results for the sputtered silicon film. (a) $3000 \mu \mathrm{N}$; (b) $6000 \mu \mathrm{N}$; (c) $9000 \mu \mathrm{N}$.

For the tests of the standard fused quartz, the reduced modulus was consistent for the three loads. The average elastic modulus was $71.8 \mathrm{GPa}$, which was very close to the standard value of $72 \mathrm{GPa}$. The actual modulus of the fused quartz was calculated using formula 4.1. $\mathrm{E}_{\mathrm{i}}$ and $v_{\mathrm{i}}$ were chosen as $1141 \mathrm{GPa}$ and 0.07 for the diamond Berkovich tip. The Poisson's ratios of fused quartz, sputtered silicon and photoresist were assumed to be $0.17,0.22$ and 0.22 , respectively. The testing results for the sputtered silicon film also showed consistent modulus. For the load of $9000 \mu \mathrm{N}$, the indentation depth reached $186 \mathrm{~nm}$, which was still less than ten percent of the thickness of the sputtered silicon film. Thus, the load level of $3000 \sim 9000 \mu \mathrm{N}$ is appropriate for the elastic modulus test.

In order to keep the indentation depth less than $200 \mathrm{~nm}, 6000 \mu \mathrm{N}$ was used for the final measurement in terms of the trial tests. Twelve indentations were made with this 
load, and the elastic modulus of the sputtered silicon film was 114.8 $\pm 2.3 \mathrm{GPa}$. Figure 4.15 shows the SPM image after one indentation test.

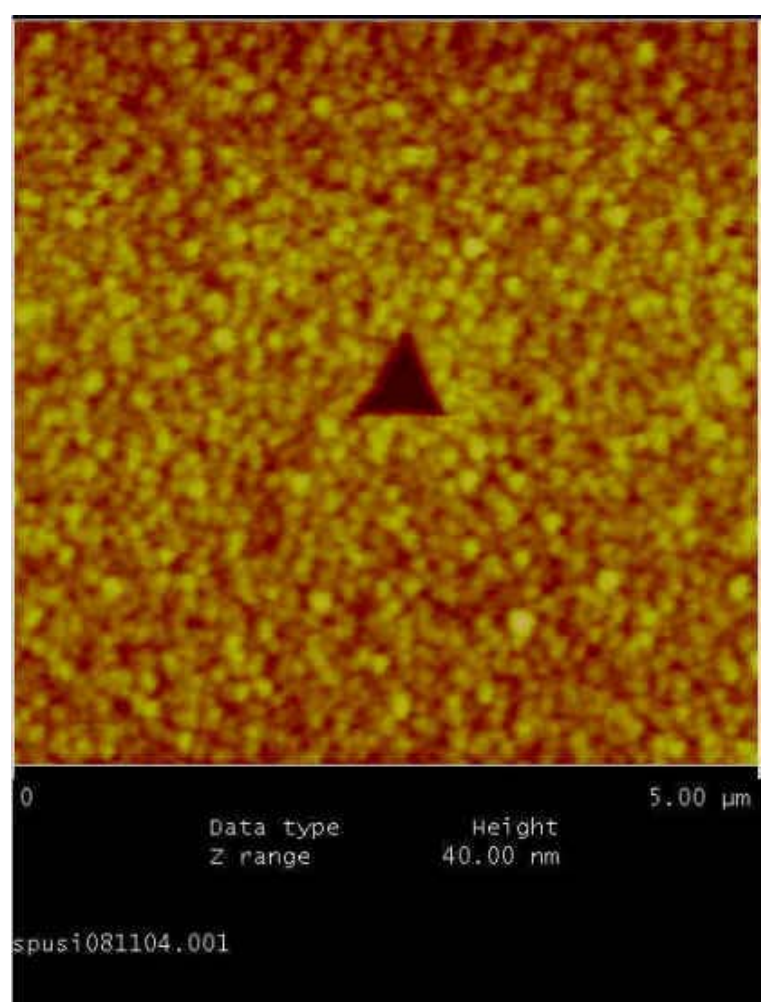

Figure 4.15 SPM image of the sputtered silicon surface after the indentation test.

The measured value is smaller than the elastic modulus of the polysilicon film, which is $150 \mathrm{GPa} \sim 160 \mathrm{GPa}$. The reason is that the oxygen impurity in the sputtered silicon film forms silicon dioxide, which scatters in the film. This will significantly lower the elastic modulus of the sputtered silicon. Therefore, the measured value of $114.8 \pm 2.3 \mathrm{GPa}$ is reasonable for the impure silicon film.

The same trial procedure was used to determine the load level for the modulus test of the baked photoresist film. In order to get relatively accurate results, the photoresist 
film was prepared with large thickness so that the substrate impact on the testing results can be eliminated. The photoresist film was directly applied on the silicon wafer. The thickness of the photoresist film lies in the range of $6 \sim 8 \mu \mathrm{m}$. The photoresist-coated wafers were loaded in the oven for soft-bake and hard-bake with a baking time of 40mins. The soft and hard bakes were conducted four times to simulate the process history experienced by the photoresist in the fabricated microstructure. After the baking processes, the wafers were diced into $0.8 \times 0.8$ chips. Figure 4.16 presents a loaddisplacement curve for one indentation test. Figure 4.17 is the SPM image of the photoresist film surface after the indentation test.

The load level shown in Figure 4.16 was used for the measurement. Ten indentations were made and the value of the elastic modulus of the photoresist film was $0.83 \pm 0.05 \mathrm{GPa}$. This value is smaller than the elastic modulus reported for thick photoresist such as SU-8, which is about $4 \sim 5 \mathrm{GPa}$. 


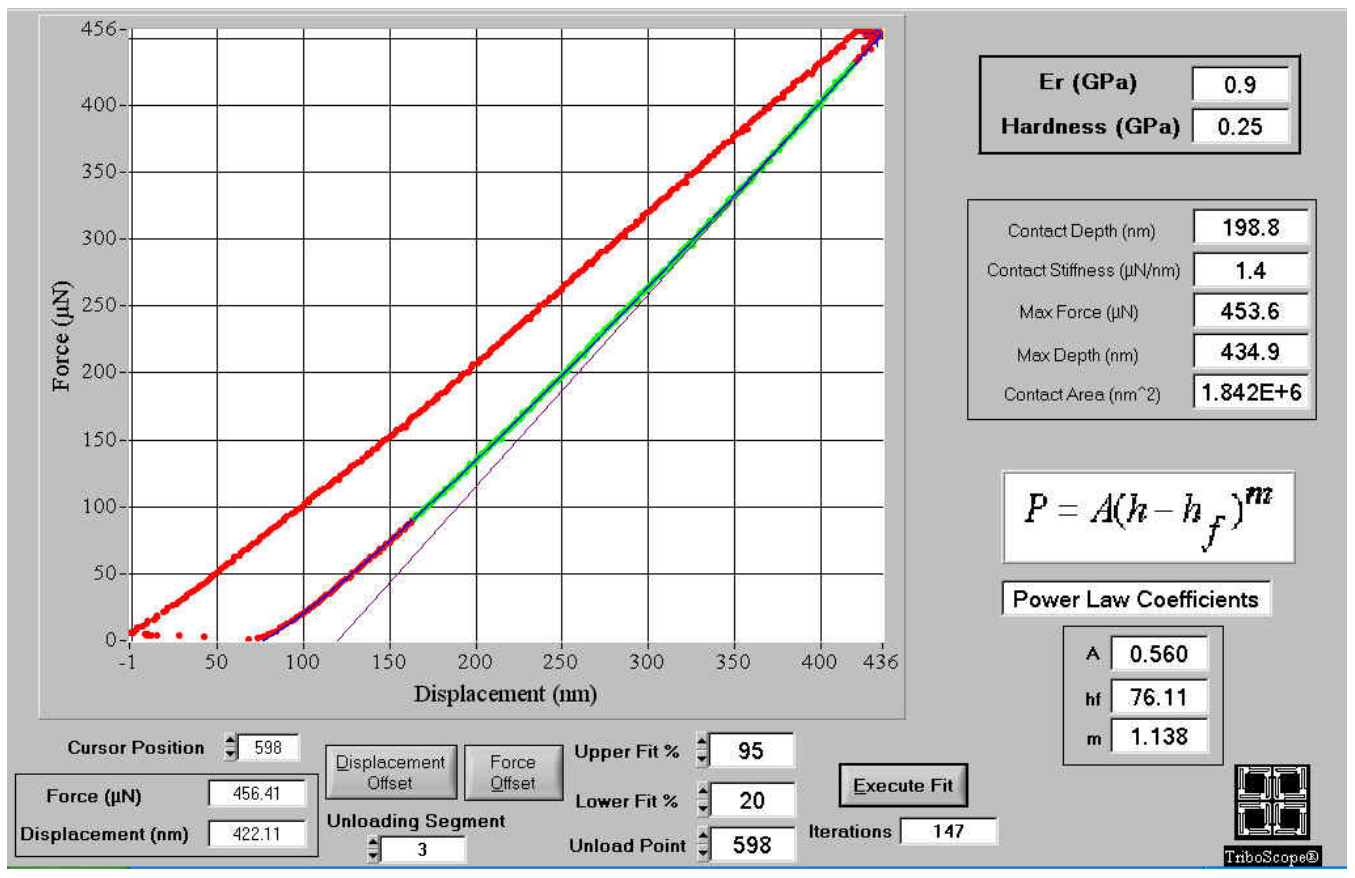

Figure 4.16 Load-displacement curve of an indentation test for the photoresist film.

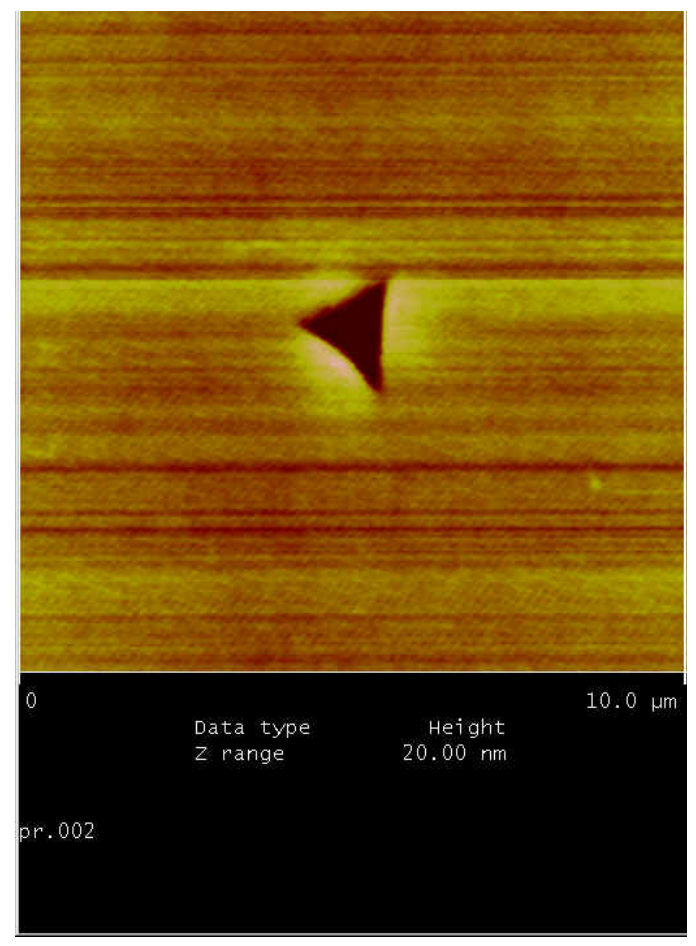

Figure 4.17 SPM image of the photoresist film surface after indentation test. 


\section{Chapter Five}

\section{Mechanical Properties of the Micro-composite Structure}

\subsection{Instrument control for data acquirement}

The mechanical property testing of the fabricated microstructures was conducted using a flat punch tip and the 2-D transducer. The flat punch tip was customized for this test from Hysitron, Inc. Figure 5.1 shows tip's side view. The flat tip is supposed to be a diamond cylinder with a cross-section diameter of $50 \mu \mathrm{m}$.

\section{Diamond flat punch tip, Diameter $50 \mu \mathrm{m}$}

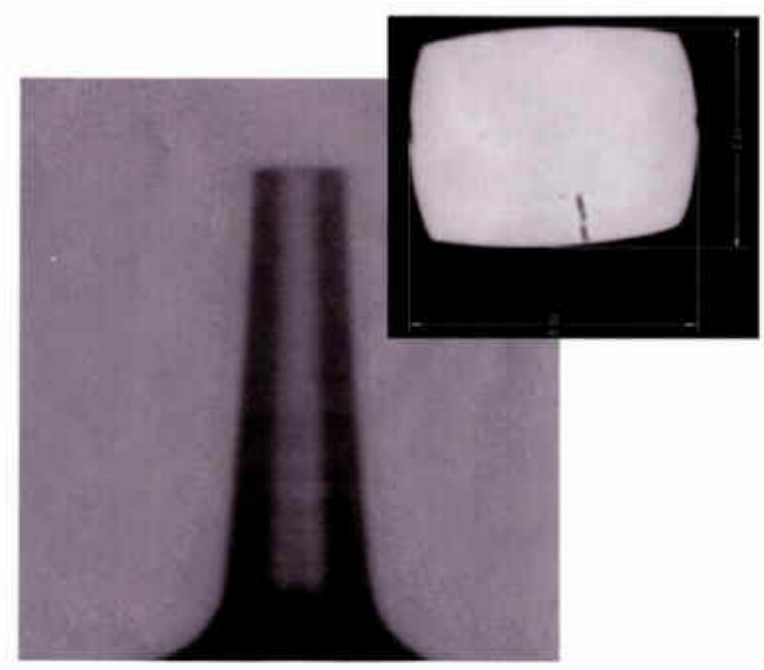

Figure 5.1 Side view of the flat punch tip. 
The actual tip sidewall is not perfectly rounded. This can be seen from the magnified cross-section of the tip's free end shown in Figure 5.2. Even though it is not perfectly round, the cross-section shape is still symmetric about the two axes across the cross-section center. The dimensions in the two symmetric axis directions are $49.3 \mu \mathrm{m}$ and $48.8 \mu \mathrm{m}$, respectively.

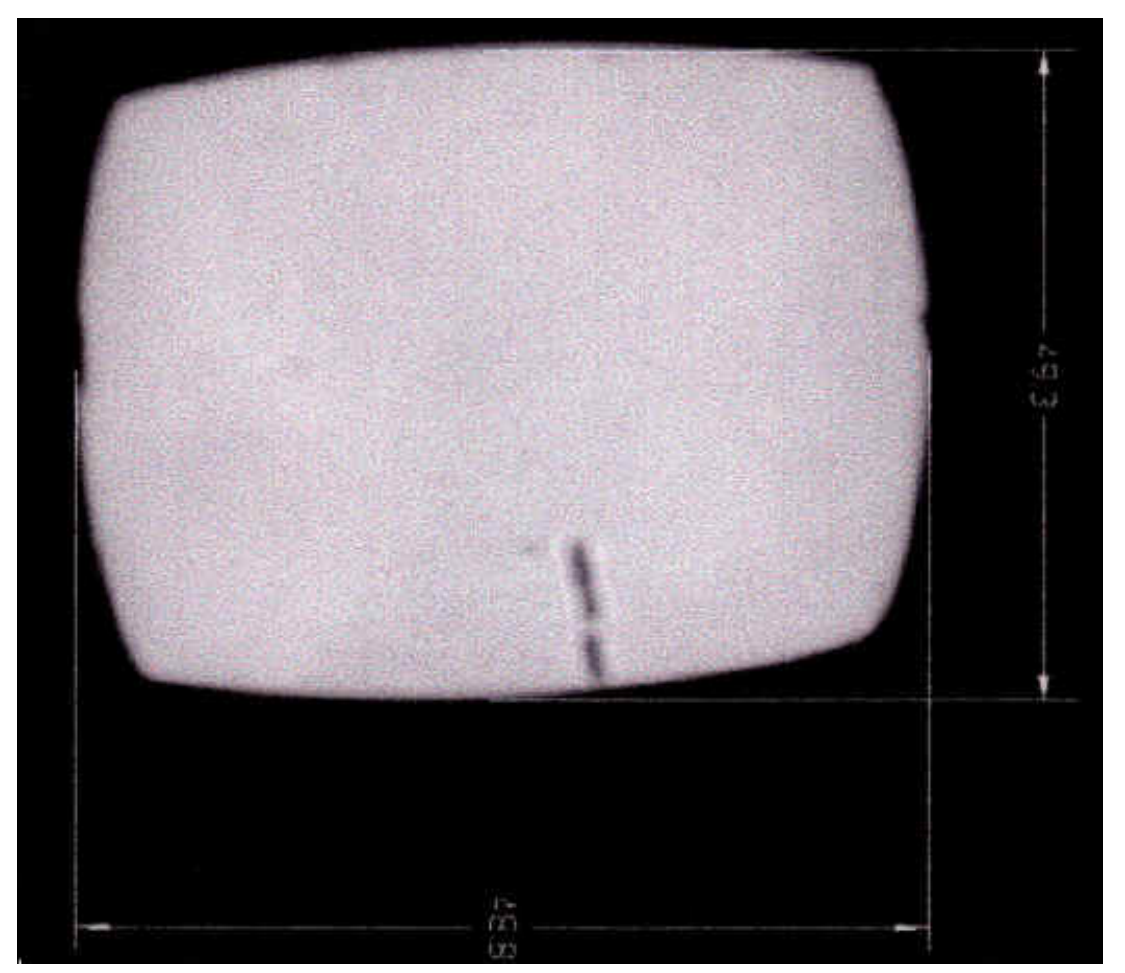

Figure 5.2 Magnified cross-section of the tip's free end.

A typical image captured through the vision system is shown in Figure 5.3. The image displaced in the video-capture window is the tip, its shadow and the nearby substrate. The one on the right is the tip and the one on the left is its shadow. The video shown in the vision window is a mirror image of the actual positions of the tip and 
specimens. If the video shows that the tip forces the beam to move from the left to the right in the vision window, the actual movement of the beam is from the right to the left.

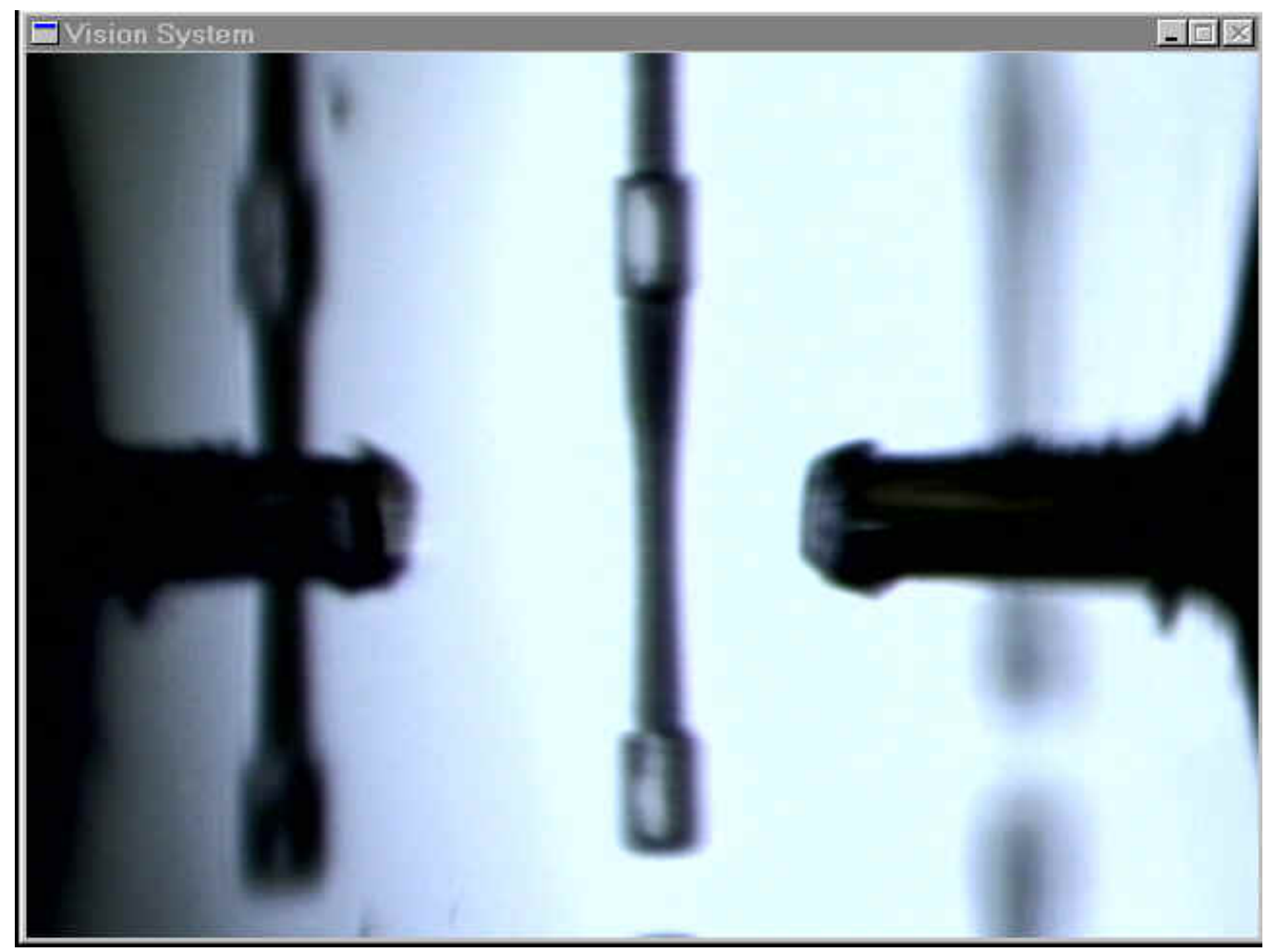

Figure 5.3 Image of the tip, its shadow and the nearby substrate captured by the vision system.

Since no in-situ visual system with high magnification is assembled on the nanoindentation system, the specimen deformation procedure cannot be clearly observed and recorded. This makes it impossible to observe what really happened in the microstructure with respect to the specific data points in the load-displacement curve. Lack of in-situ visual system will also cause difficulties in locating the tip right in front of the microstructure. The only way to tell weather the tip starts contacting the specimen is to make a scratch test after locating and engaging the tip. During the scratch test, the 
software will synchronously display the data collection of the normal displacement versus the lateral displacement. If a rapid change of the normal displacement appears within a short time, it is assumed that the tip has already contacted the specimen. This tip positioning procedure is explained in detail as follow:

1. Attach the flat punch tip to the 2-D transducer, assemble the 2-D transducer on Triboscope scanner, and fix the Triboscope scanner onto the SPM stage frame.

2. Mount the sample chip on SPM stage, use the vision system to find the tip and lower it towards the chip substrate until it is about $15-20 \mu \mathrm{m}$ above the substrate. Figure 5.3 shows what it looks like at this point.

3. Set up the lateral load function. Since the lateral test uses the displacement control, the actual controlling function is a displacement function. A typical displacement function used for this test is shown in Figure 5.4. This displacement function has five segments, and the duration time from the first segment to the fifth segment is 5 secs, 5secs, 30secs, 5secs and 10secs, respectively. The tip will move backward for $1 \mu \mathrm{m}$ in the first $5 \mathrm{secs}$ and hold there for $5 \mathrm{secs}$, then move forward $11 \mu \mathrm{m}$ within 30 secs and hold there for $5 \mathrm{secs}$. Eventually, the tip will move back to its original position within $10 \mathrm{secs}$. No normal force is applied during the lateral test. 


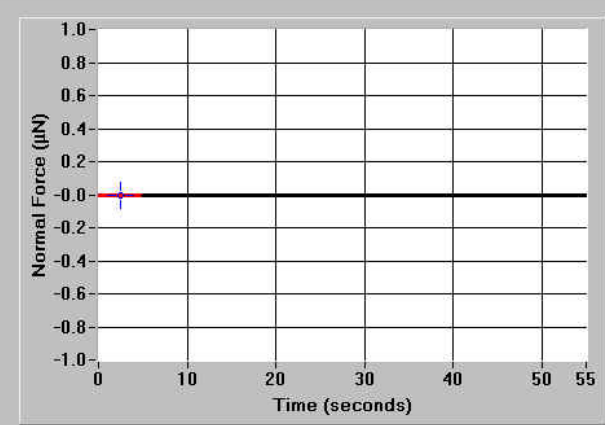

Time (seconds)

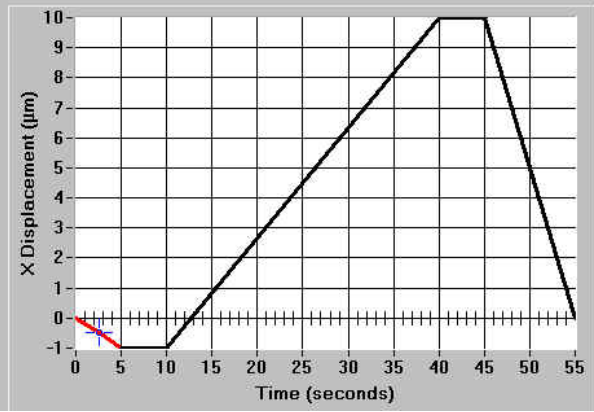

Segment *

Segment Time (seconds)

Begin Time (sec)

End Time (s)

Begin Force $(\mu \mathrm{N})$

End Force $(\mu N)$

Peak Force $(\mu \mathrm{N})$

1 $\leqslant \sqrt{5.00}$ 0.00 5.00 * 0.00 * 0.00 $\div 0.00$

Begin Displacement $(\mu \mathrm{m}) \div \sqrt{0.00}$ End Displacement $(\mu \mathrm{m}) \quad * \sqrt{-1.00}$ Max Displacement $(\mu \mathrm{m}) \quad \sqrt[4]{10.00}$ Min Displacement $(\mu \mathrm{m}) \quad \div \quad \sqrt{-1.00}$ of Data Points $\sqrt[4]{1000}$

t to Average $\sqrt[4]{1}$

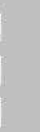

Figure 5.4 Displacement control function.

4. Calibrate the tip in the air using the displacement function shown in step 3. This operation will define the 2-D transducer constants. Figure 5.5 shows the collected data for the third scratch segment. Because the scratch is not on a sample, the data in the lateral force/time window should be scattered about zero. Due to the limitation of the spring system in the 2-D transducer, the lateral displacement has its upper limit, which is about $10 \mu \mathrm{m}$. Beyond this limit, the calibrated lateral forces will deviate from zero. This can be used as a criterion to tell weather the lateral displacement is still in the valid region. 

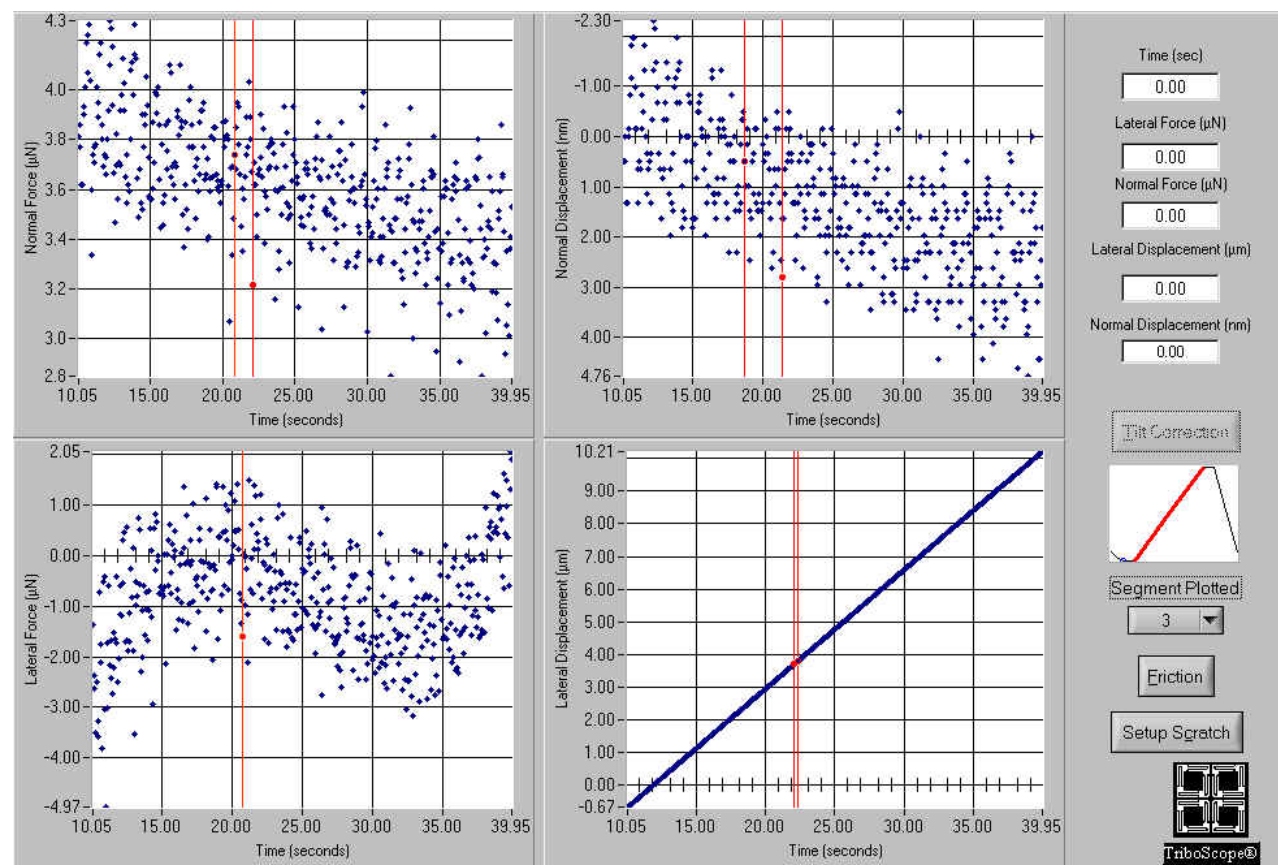

Figure 5.5 Calibration of the lateral force.

5. Locate the tip close to the center of the beam. Since there is no scalar in the vision screen and no distance control in the tracking ball system, the central position is visually estimated. The tip is engaged in the middle layer side so that the bending load will generate tensile stress in the inner layer side.

6. Engage the tip to contact the substrate. The image of this engaged tip is shown in Figure 5.6. Once the tip is engaged, the scan size has to be set to keep the tip scanning on the substrate. Due to the unpredictable distance between the tip and the beam, the scan size is set to be a few nanometers to ensure no contact between them. 


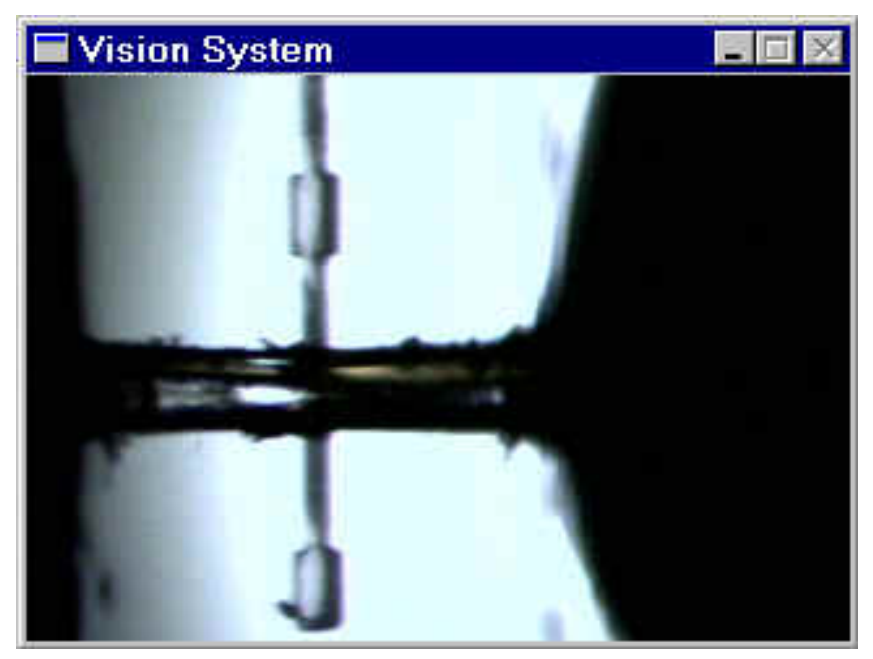

Figure 5.6 Engaged tip.

7. Start a scratch test using the displacement function shown in step3. The data collection window shows data points of the normal displacement versus the lateral displacement. If there is no contact between the tip and the specimen, the normal displacement should be smaller than $5 \mathrm{~nm}$ and the data points scatter within the band of $0 \sim 5 \mathrm{~nm}$. If the tip starts touching the specimen, the normal displacement will rapidly deviate from zero and the test will be aborted immediately. The distance that the tip has already traveled before touching the specimen will be recorded and the tip is moved towards the specimen with the same distance so that it is located right in front of the specimen. If after one displacement function the contact position is still not found, the tip will be moved $10 \mu \mathrm{m}$ towards the specimen for starting a new scratch test. This operation will be repeated until the contact position is found. The SPM software controller can only move the tip $\pm 50 \mu \mathrm{m}$ from its engaging position. If after $50 \mu \mathrm{m}$ of movement the contact position is still not found, the tip has to be disengaged. The tracking ball system will be used to move the tip towards the 
specimen a certain distance and then repeat the procedure from step5 until the contact position is found.

Once the contact position is found, the beam-bending test will be conducted and the lateral and normal load-displacement data will be recorded.

\subsection{Mechanical properties of the micro-composite structure}

A typical released beam is shown in Figure 5.7. Good releasing results were only achieved with $200 \mu \mathrm{m}$ long beams. A large number of $300 \mu \mathrm{m}$ long beams stuck on the substrate after being released. Therefore, only $200 \mu \mathrm{m}$ long beams were chosen for the mechanical property test.

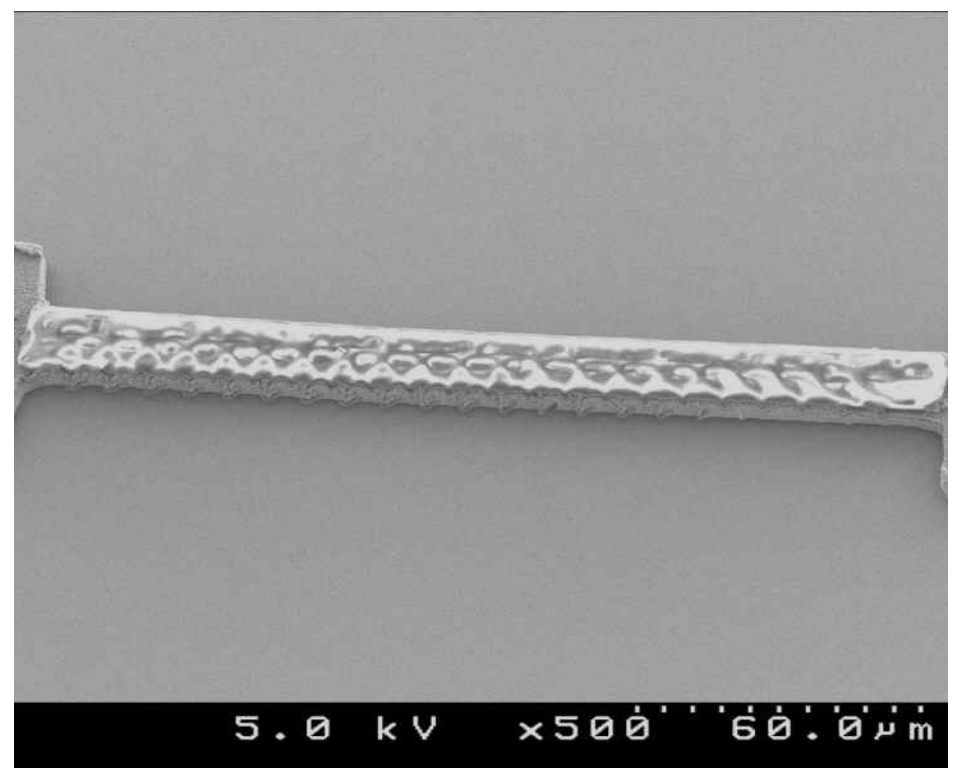

(a) 


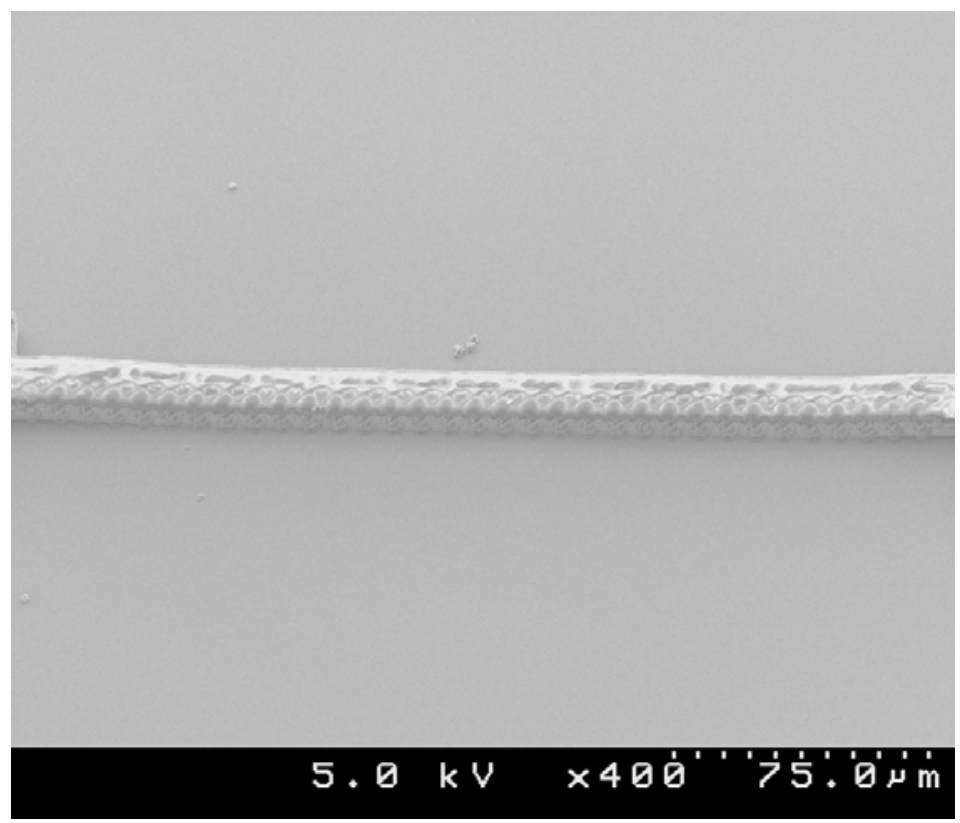

(b)

Figure 5.7 Released beams. (a) $200 \mu \mathrm{m}$ long beam; (b) $300 \mu \mathrm{m}$ long beam.

\subsubsection{Load-displacement curve and structural flexural strength}

During the test, the normal force and the normal displacement were also captured. Figure 5.8 shows the representative curves of the normal force and the normal displacement versus the lateral displacement.

The normal displacements of the tip are less than $0.15 \mu \mathrm{m}$ and the normal forces are less than $100 \mu \mathrm{N}$. Since no normal force was applied on the tip and the tip was free in the normal direction, the normal force appeared here must be caused by the friction between the tip and the specimen sidewall. As seen in Figure 5.1, the sidewall of the tip end is not perfectly straight, and it is impossible for the etching process to produce a perfectly straight sidewall for the specimens. These factors make the friction force possible. 
The vertical shift of the tip against the specimen is only $1 \%$ of the lateral displacement. Using the finite element model employed in Chapter 2 and the measured elastic moduli of the sputtered silicon and the photoresist, the normal displacement of the specimen was calculated with the maximum normal load of $100 \mu \mathrm{N}$ as shown in Figure 5.8. The result showed that the normal displacement was smaller than $0.2 \mu \mathrm{m}$. Because the suspending distance between the specimen and the substrate is $2 \mu \mathrm{m}$, the normal force is too small to make the specimen touch the substrate.

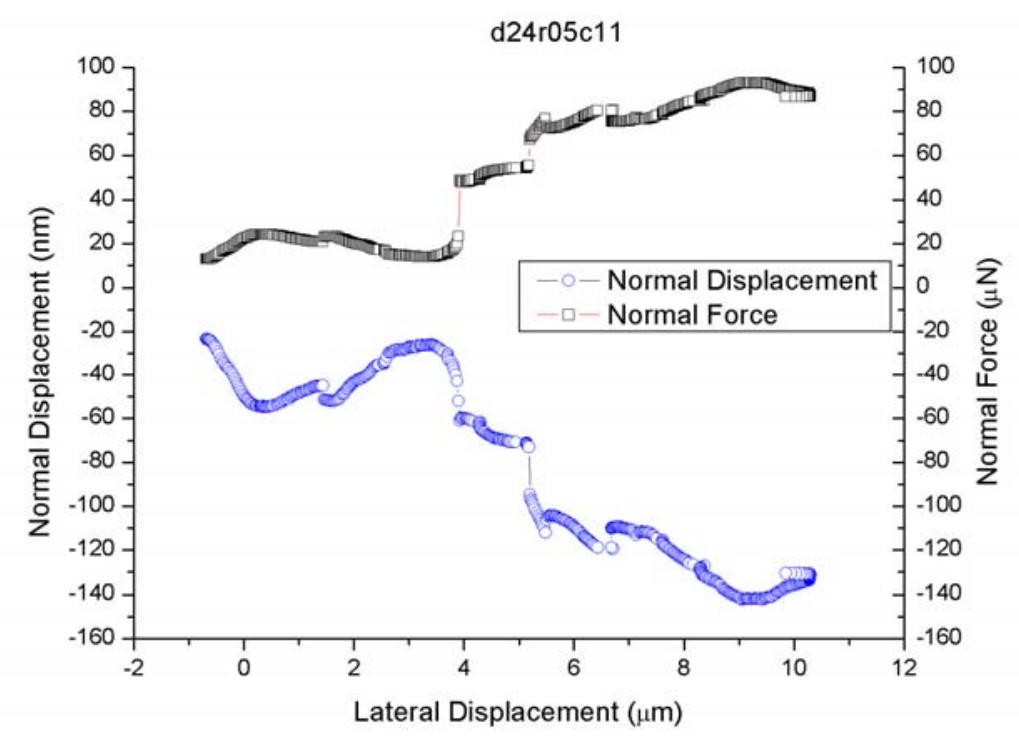

Figure 5.8 Curves of the normal force and normal displacement versus the lateral displacement.

The normal force is an eccentric load with respect to the symmetric axis of the beam and exerts torque on the beam. This will force the beam to twist with respect to the beam length direction and introduce shear stress in the cross-section. As seen in Figure 5.8, the normal force is very small within the elastic deformation region. It cannot 
significantly affect the global deformation. With the increment of lateral displacement, the normal force may reach a relatively large number at some point. Due to the nature of highly nonlinear deformation, the torsion effect cannot be simply superimposed to the lateral deformation. Moreover, the normal force randomly varies with the increment of the lateral displacement and no clue is available for tracking its variation trend. Therefore, only lateral force will be used for evaluating the mechanical properties of the microstructures. This simplification is reasonable since the ratio of the width over the depth is only about 3 and the torsion-caused deformation is not very prominent compared to the lateral deformation.

Two factors might affect the accuracy of the testing data and need to be compensated to the lateral forces and lateral displacements. One is the friction between the flat punch tip and the substrate. Another is the self-deformation of the tip.

In order to measure the friction between the flat punch tip and the substrate, a scratch test was conducted after engaging the tip. The tip was engaged at the location where no contact could happen between the tip and the microstructures. Figure 5.9 shows the testing result for the scratch test. 


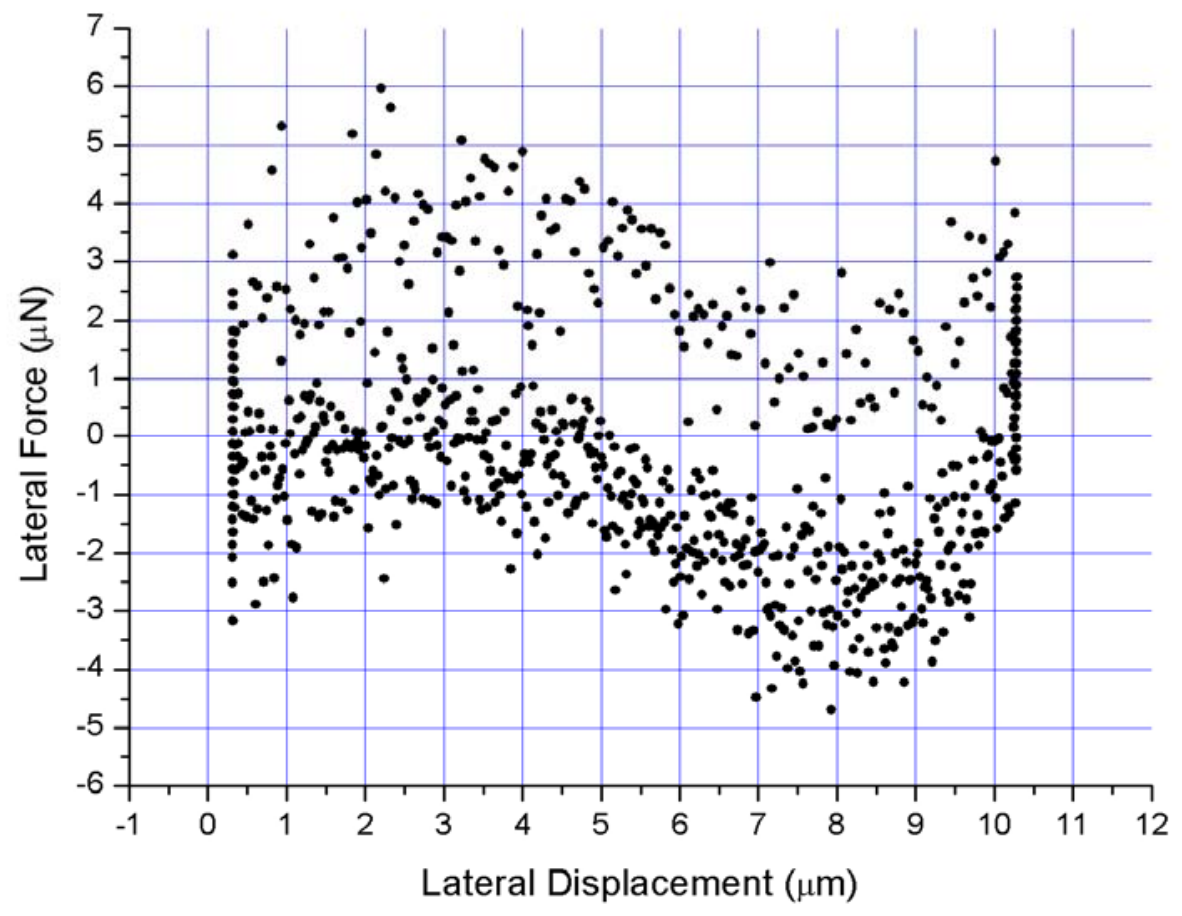

Figure 5.9 Data points of the friction versus the lateral displacement.

It can be seen that the lateral force, which is friction in this case, is almost zero and the maximum force is still less than $5 \mu \mathrm{N}$. It can be ignored compared to the lateral force applied on the microstructures. This simplification is also based on the assumption that the surface roughness of the substrate material is consistent at different locations.

The self-deformation of the tip was simulated using ABAQUS with the dimensions shown in Figure 5.1. The diamond tip was simplified as a cantilever beam with a circular cross-section. The beam length and cross-section radius were $238 \mu \mathrm{m}$ and $49.3 \mu \mathrm{m}$, respectively. Figure 5.10 shows the load-displacement curve for the free end of the tip. 


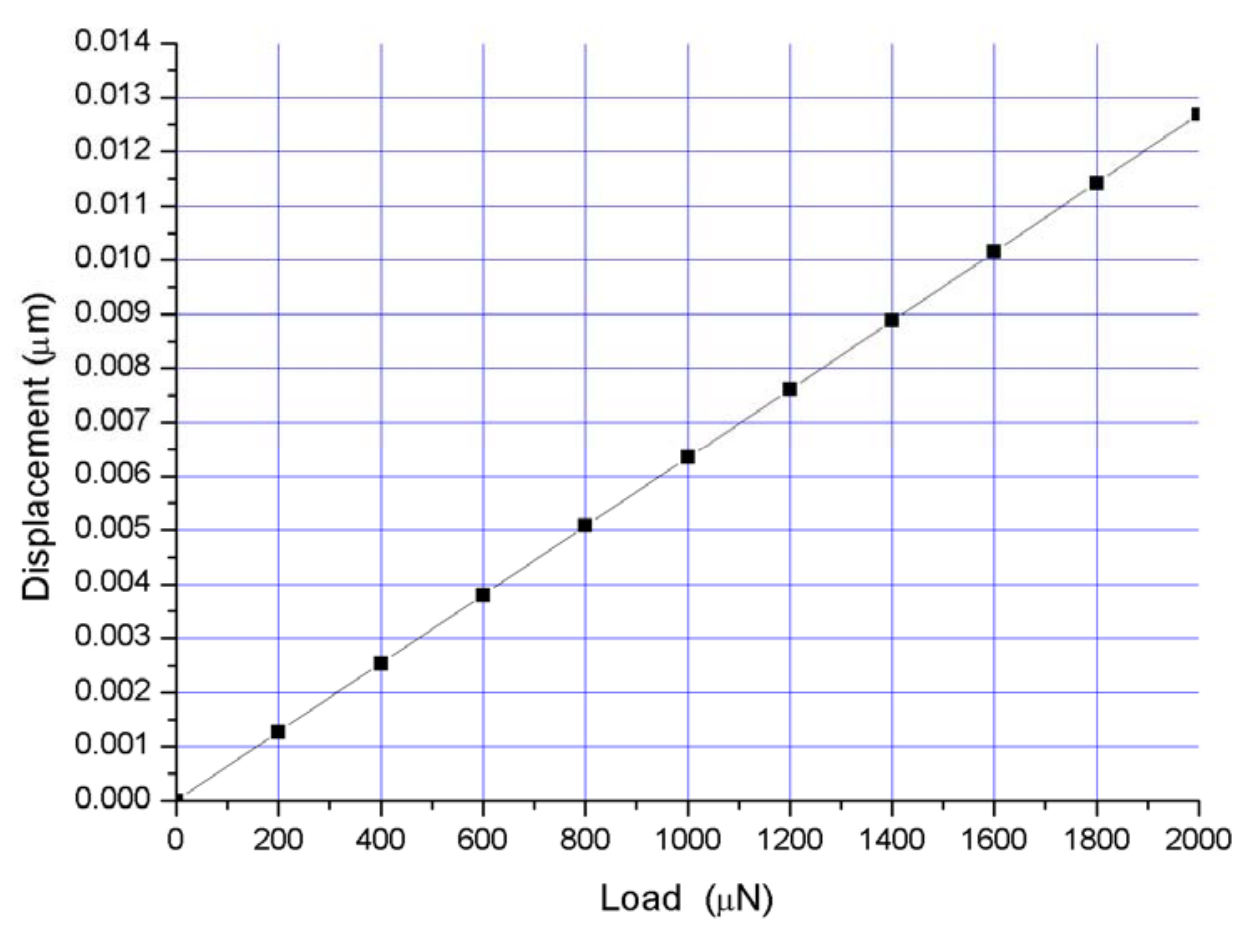

Figure 5.10 Load-displacement curve of the free end of the flat punch tip.

Corresponding to the maximum load of $1500 \mu \mathrm{N}$, the displacement of the tip is smaller than $0.01 \mu \mathrm{m}$. The effective stiffness of the flat punch tip is $160000 \mathrm{~N} / \mathrm{m}$. Compared to the average effective stiffness of the specimen, which is about $2000 \mathrm{~N} / \mathrm{m}$, the effective stiffness is large enough for the tip to be considered as a rigid body.

Based on the results presented above, the data collected from the lateral testing can be directly used to analyze the mechanical behaviors of the microstructures. Among the tested beams, 11 beams, which show consistent behaviors, were used for the analysis.

Figure 5.11 shows 11 load-displacement curves for the $20 \mu \mathrm{m} \times 200 \mu \mathrm{m}$ beams. Here, the label $\mathrm{d}^{* *} \mathrm{r} * * \mathrm{c}^{* *}$ represents the specimen location on the wafer. 


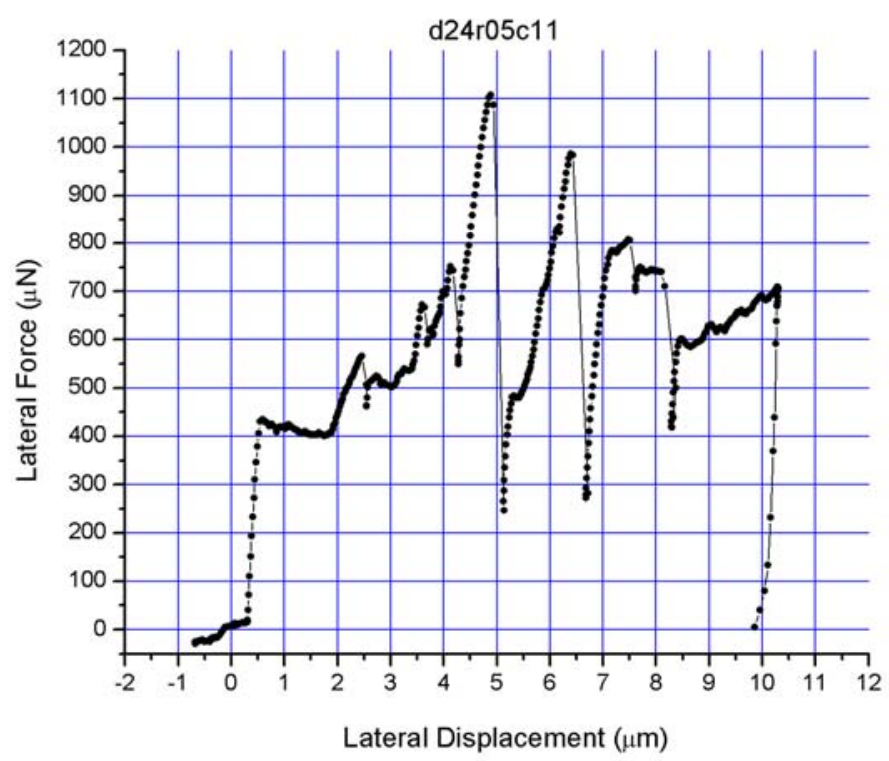

Figure 5.11--(1)

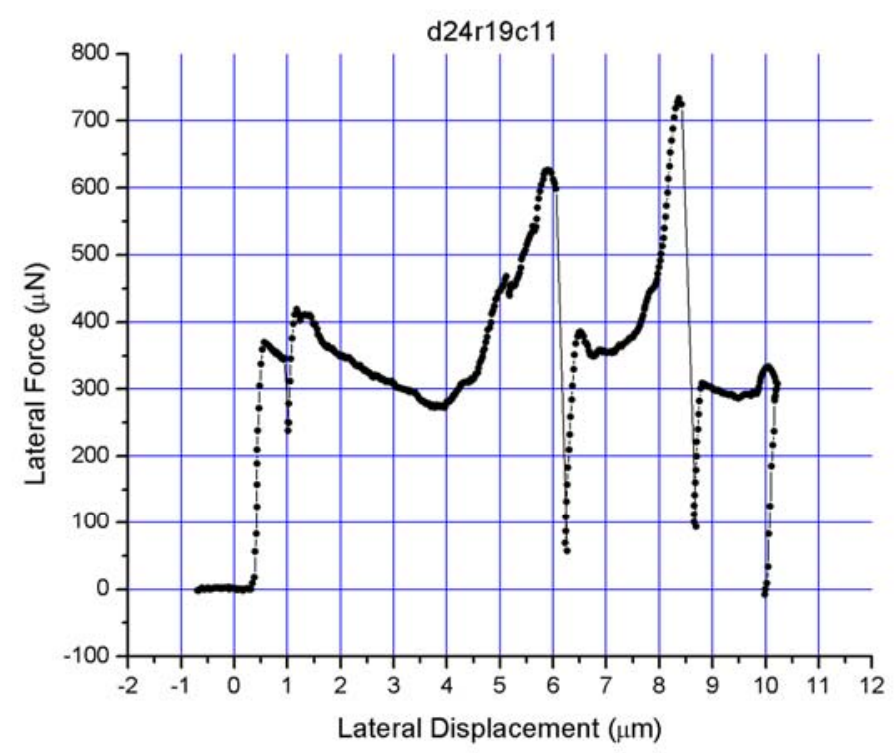

Figure 5.11-- (2) 


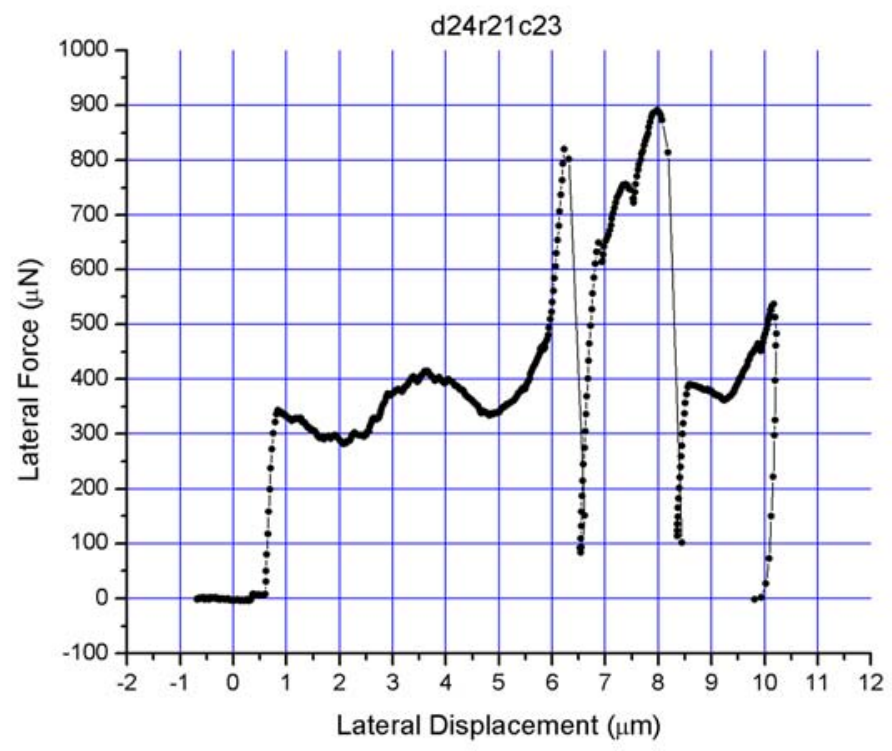

Figure 5.11-- (3)

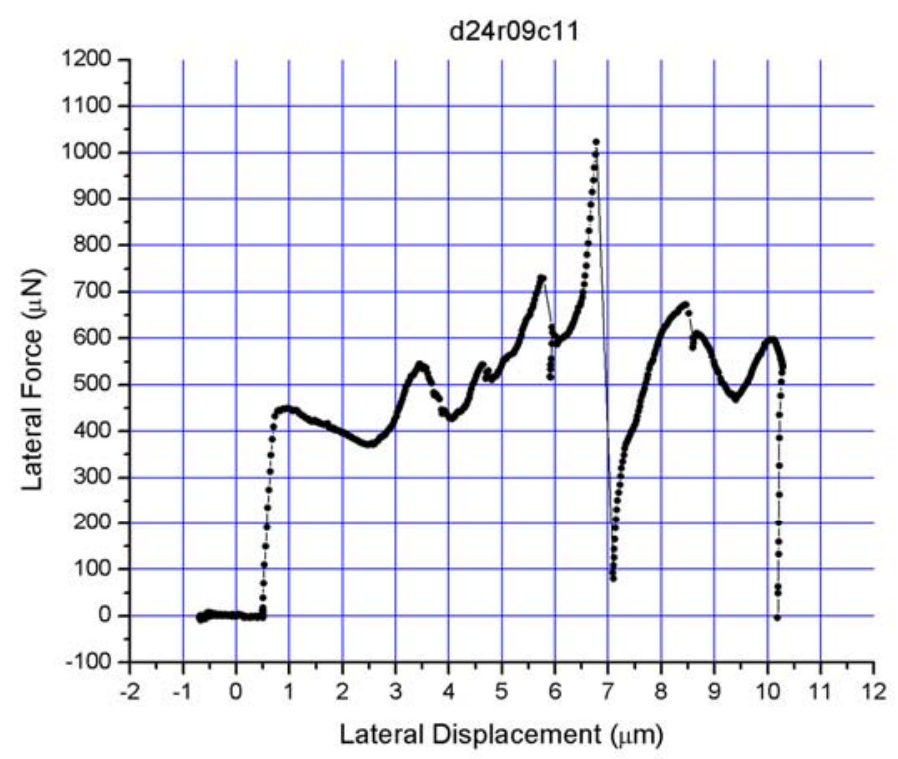

Figure 5.11-- (4) 


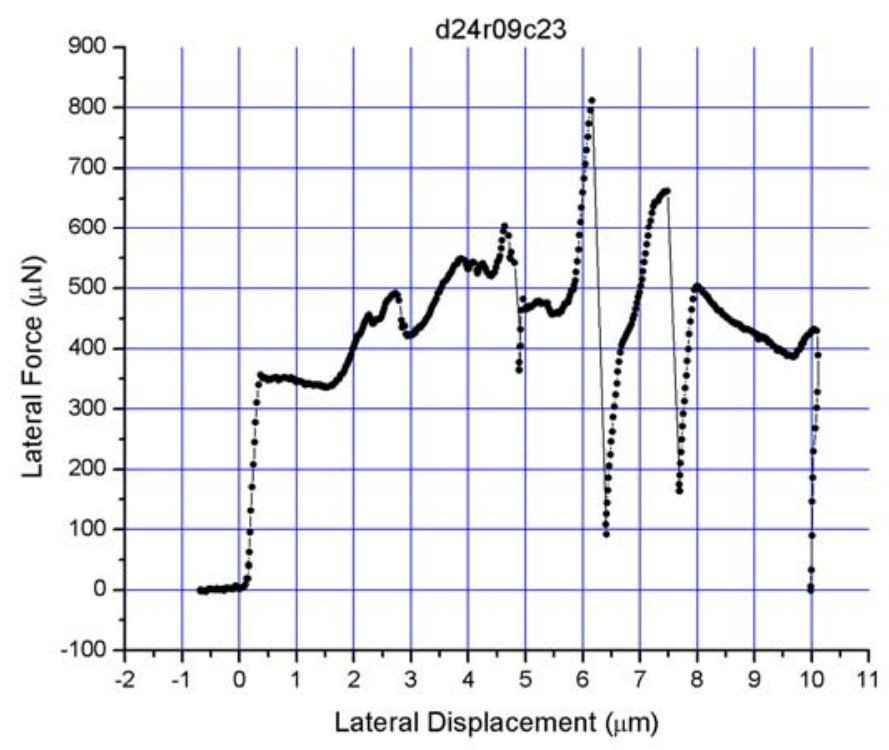

Figure 5.11-- (5)

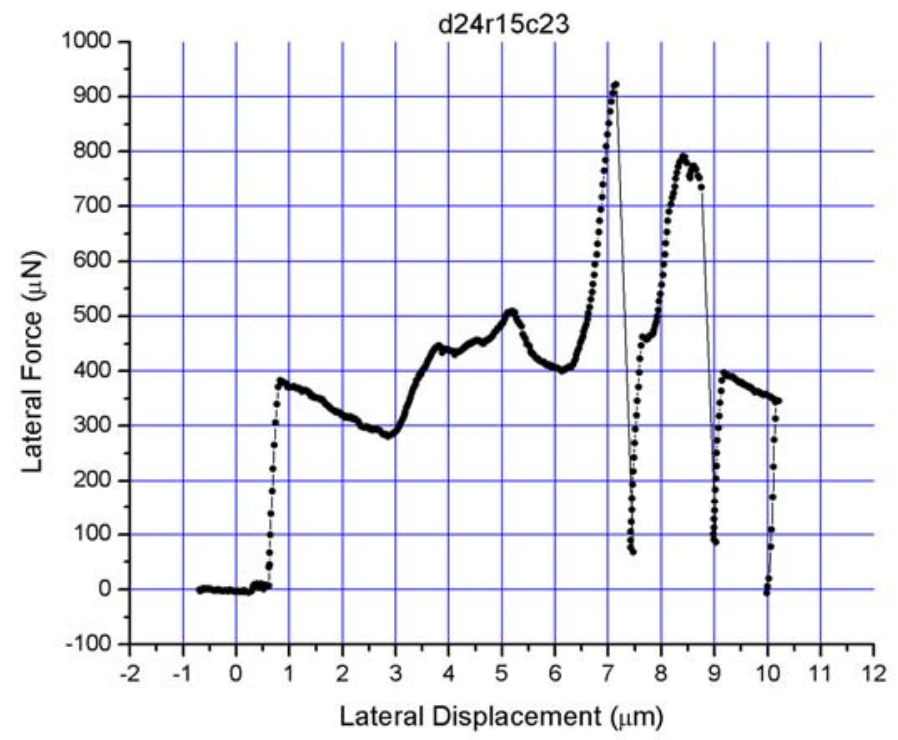

Figure 5.11-- (6) 


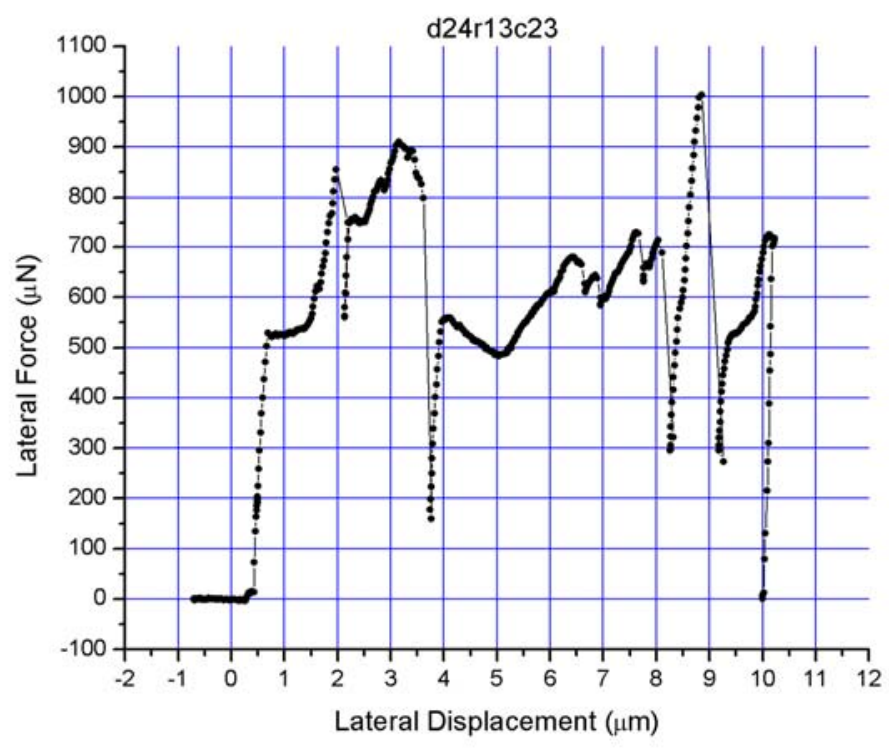

Figure 5.11-- (7)

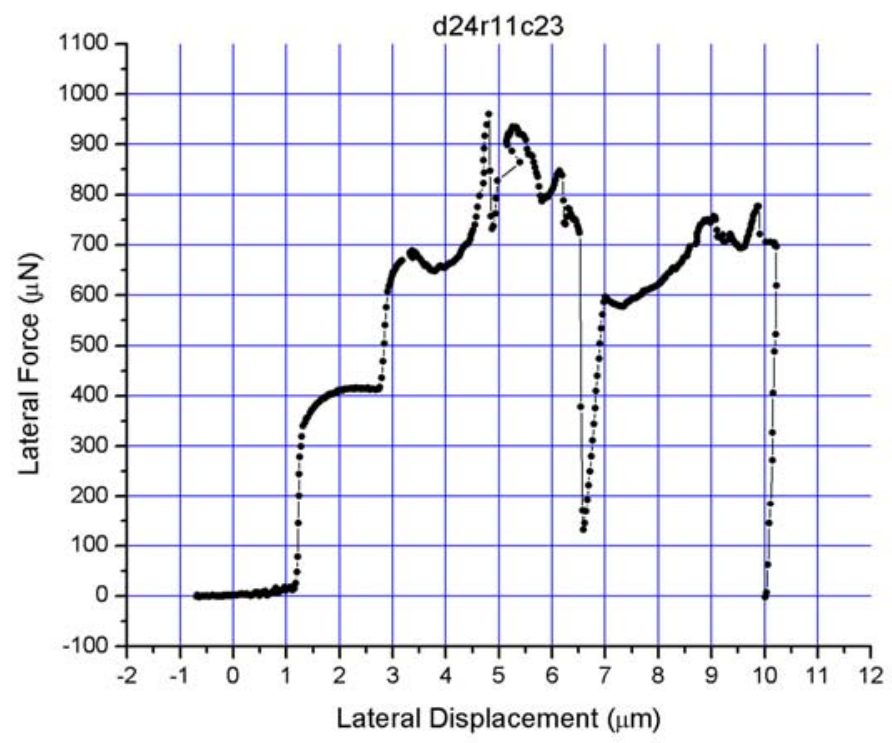

Figure 5.11-- (8) 


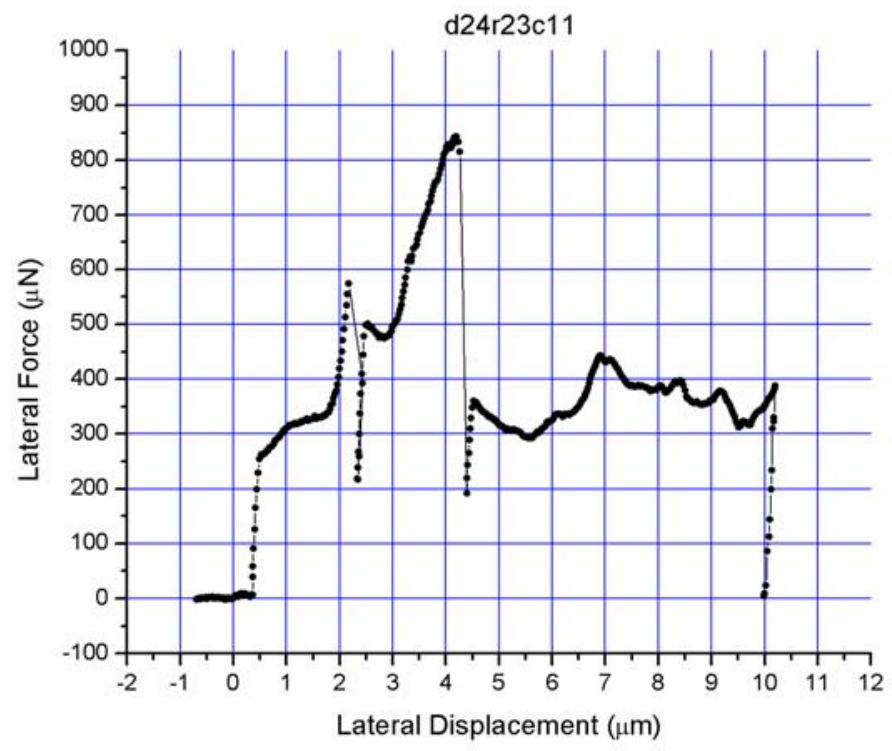

Figure 5.11-- (9)

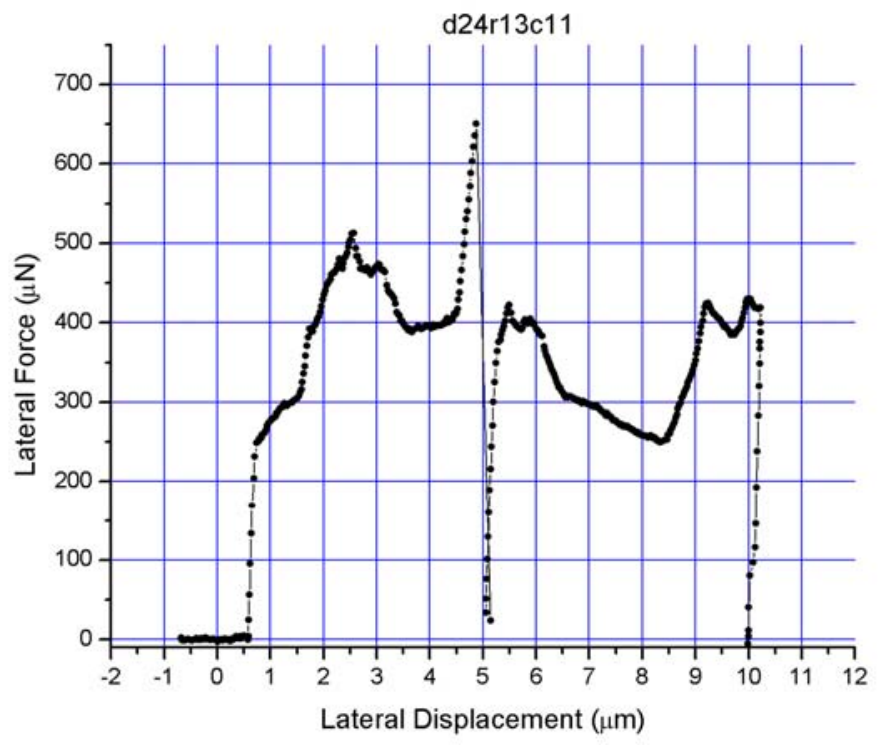

Figure 5.11-- (10) 


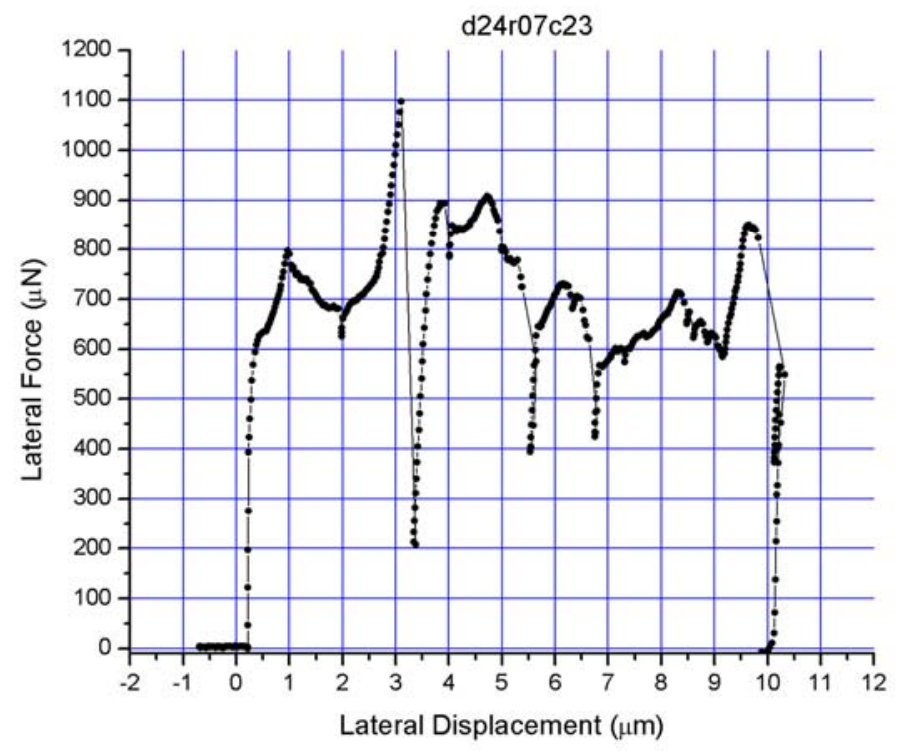

Figure 5.11-- (11)

Figure 5.11 Lateral load-displacement curves for the $20 \mu \mathrm{mx} 200 \mu \mathrm{m}$ beams.

The load-displacement curves indicate elastic deformation parts ending with obvious yield points. These yield points were used to determine the flexural strengths of the beams. For the elastic deformation, the beam was considered as a homogeneous material. The classic beam theory was used to calculate the stress at the yield point. This stress was the flexural strength of the beam.

For a double-clamped beam with a concentrated load at center, the maximum stress in the beam is calculated as follows:

$$
\sigma=\frac{M d}{I}=\frac{P L d}{8 I}
$$

Where ' $\mathrm{P}$ ' is a concentrated load, ' $\mathrm{L}$ ' is the beam span, ' $\mathrm{d}$ ' is the half beam width and 'I' is the moment of inertia. 


\begin{tabular}{|c|c|c|c|c|}
\hline Label & $(\mu \mathrm{N})$ & Point $(\mu \mathrm{m})$ & $(\mathrm{N} / \mathrm{m})$ & $(\mathrm{MPa})$ \\
\hline $\mathrm{d} 24 \mathrm{r} 09 \mathrm{c} 11$ & 443.16 & 0.273 & 1621.51 & 12.78 \\
\hline $\mathrm{d} 24 \mathrm{r} 13 \mathrm{c} 11$ & 253.83 & 0.206 & 1230.39 & 7.32 \\
\hline $\mathrm{d} 24 \mathrm{r} 05 \mathrm{c} 11$ & 435.33 & 0.276 & 1576.71 & 12.56 \\
\hline $\mathrm{d} 24 \mathrm{r} 07 \mathrm{c} 23$ & 617.37 & 0.183 & 3371.76 & 17.81 \\
\hline $\mathrm{d} 24 \mathrm{r} 09 \mathrm{c} 23$ & 356.34 & 0.287 & 1243.77 & 10.28 \\
\hline $\mathrm{d} 24 \mathrm{r} 11 \mathrm{c} 23$ & 346.47 & 0.199 & 1737.56 & 9.99 \\
\hline $\mathrm{d} 24 \mathrm{r} 13 \mathrm{c} 23$ & 528.59 & 0.277 & 1911.72 & 15.25 \\
\hline $\mathrm{d} 24 \mathrm{r} 15 \mathrm{c} 23$ & 382.94 & 0.210 & 1822.66 & 11.05 \\
\hline $\mathrm{d} 24 \mathrm{r} 21 \mathrm{c} 23$ & 342.77 & 0.233 & 1471.75 & 9.89 \\
\hline $\mathrm{d} 24 \mathrm{r} 23 \mathrm{c} 11$ & 262.12 & 0.168 & 1560.24 & 7.56 \\
\hline $\mathrm{d} 24 \mathrm{r} 19 \mathrm{c} 11$ & 369.73 & 0.231 & 1601.95 & 10.67 \\
\hline mean & 394.42 & 0.231 & 1740.91 & 11.38 \\
\hline STD & 107.79 & 0.042 & 580.43 & 3.11 \\
\hline
\end{tabular}

Table 5.1 Flexural strengths of the beams.

Table 5.1 shows the calculating results and the statistic values for 11 specimens. The average flexural strength is $11.38 \pm 3.11 \mathrm{MPa}$, which is relatively low. This is due to either the low photoresist strength or the low interface strength.

In Figure 5.11 (1)-(6), the yield point is followed by material softening, which means that damage has been introduced in the microstructure. It is believed that the damage corresponds to the interface split between the photoresist and the sputtered silicon. Due to the fabrication nature, it is very likely for the fabrication processes to 
leave defects at the interface and form imperfect bonding. This will significantly lower the structural strength.

In Figure 5.11 (7)-(11), no material softening appears right after the yield point. Beyond the yield point, plastic deformation, which is mainly conducted by the photoresist, arises in the microstructure. In these beams, the interface bonding is relatively strong compared to the photoresist strength. Actually, the probability of forming imperfect interface bonding is very high and in most of the cases, the elastic deformation is terminated by the interface split. Therefore, it is believed the low flexural strength is a reflection of the weak interface bonding.

\subsubsection{Tunnel cracking and crack bridging mechanisms in the microstructure}

Figure 5.12 shows the SEM image for beam-d24r15c23 and its load-displacement curve. It can be clearly seen that the crack starts from the interface region and no obvious damage exist in other parts. This proves that the average value shown in Table 4.1 is the strength of the interface bonding. 

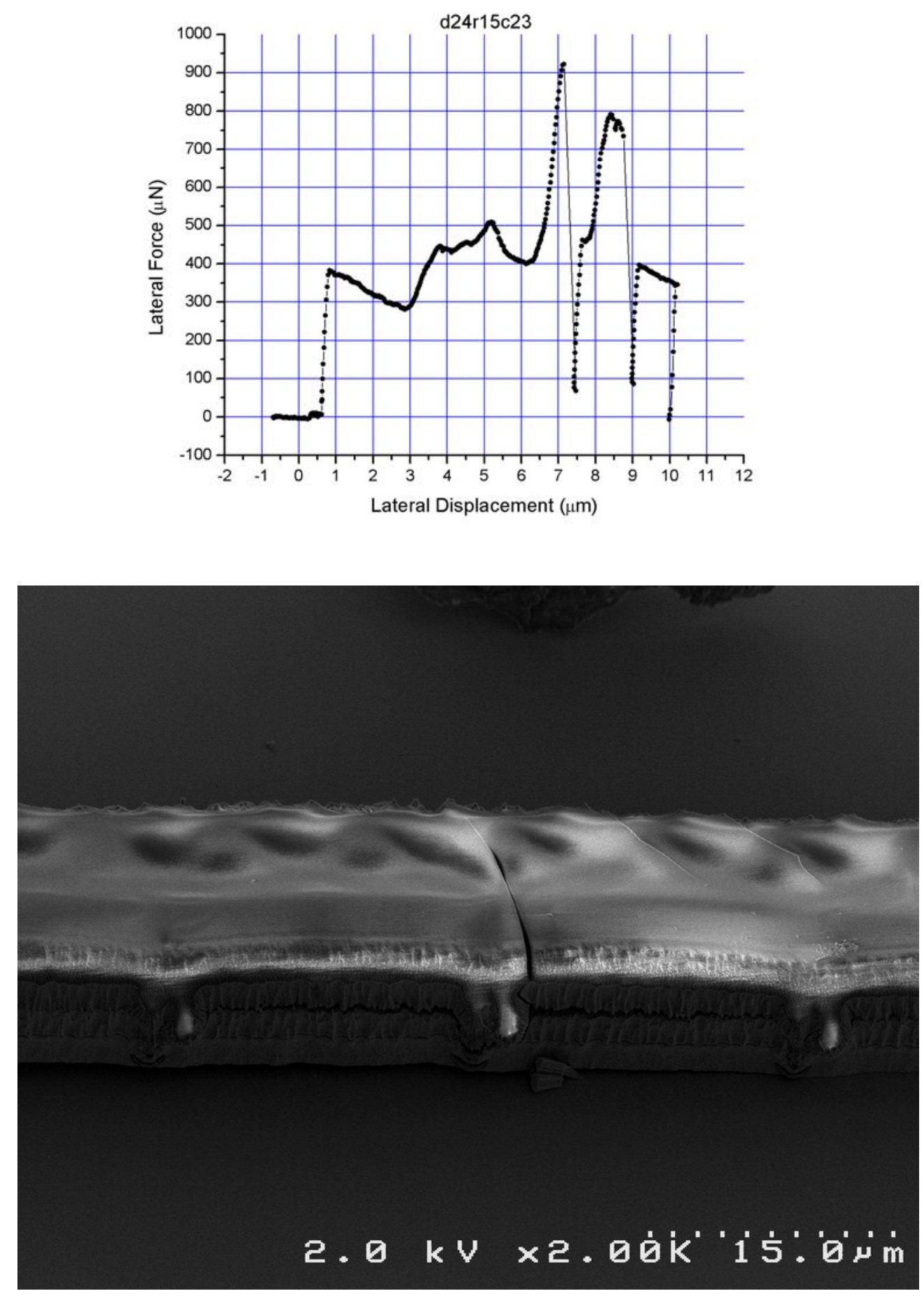

Figure 5.12 SEM image of the beam--d24r15c23 after the bending test and the corresponding load-displacement curve. 


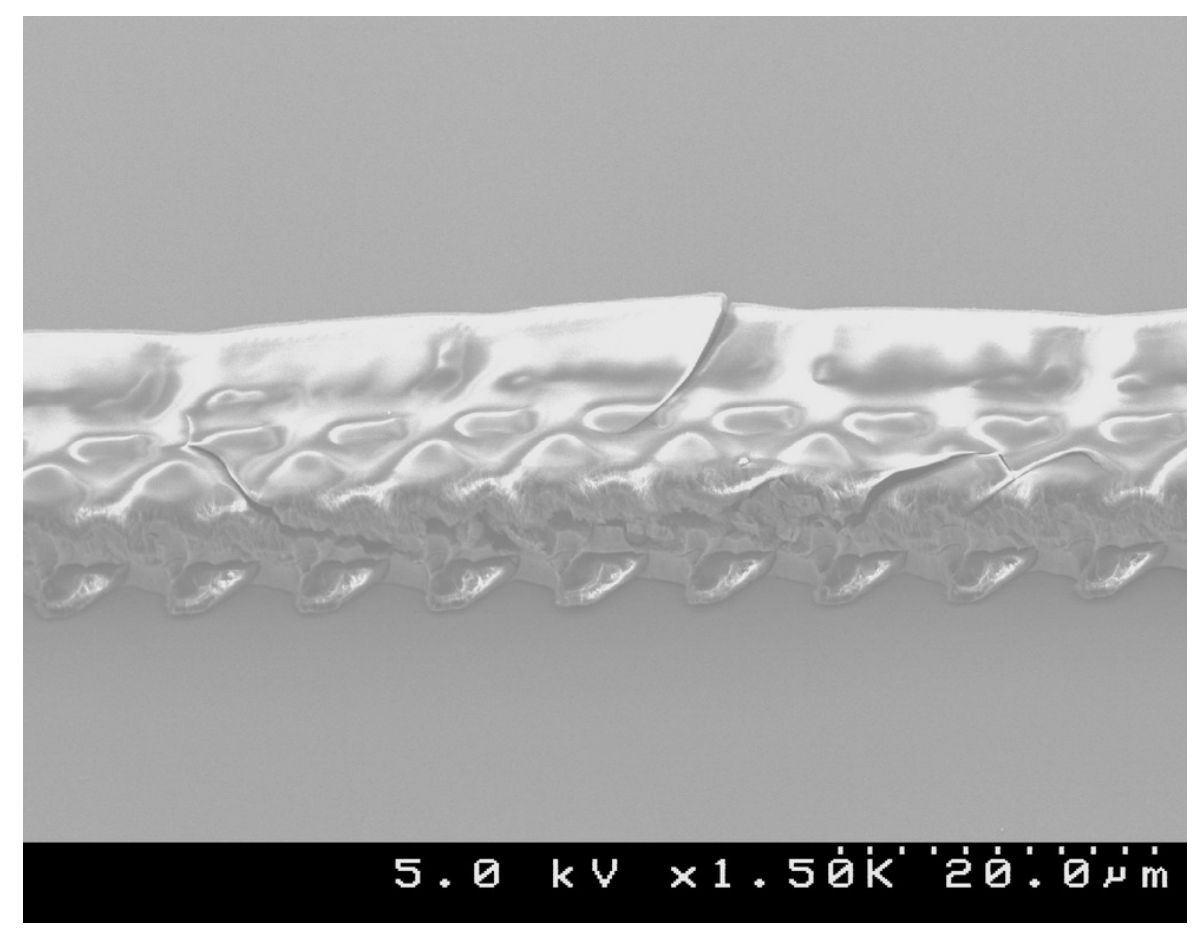

(a)

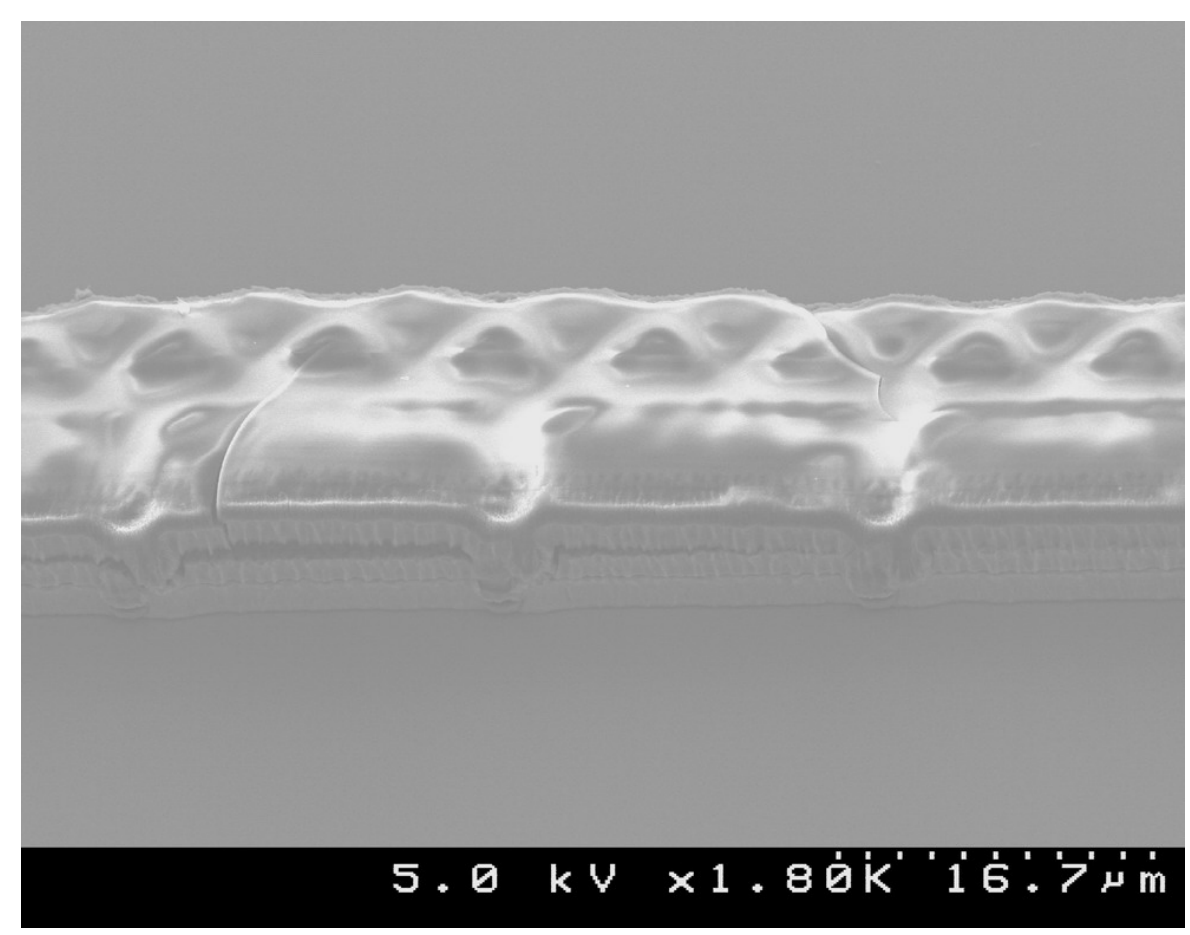

(b)

Figure 5.13 SEM images of the beam-d24r13c11 after the bending test. (a) Middle layer side; (b) Inner layer side. 


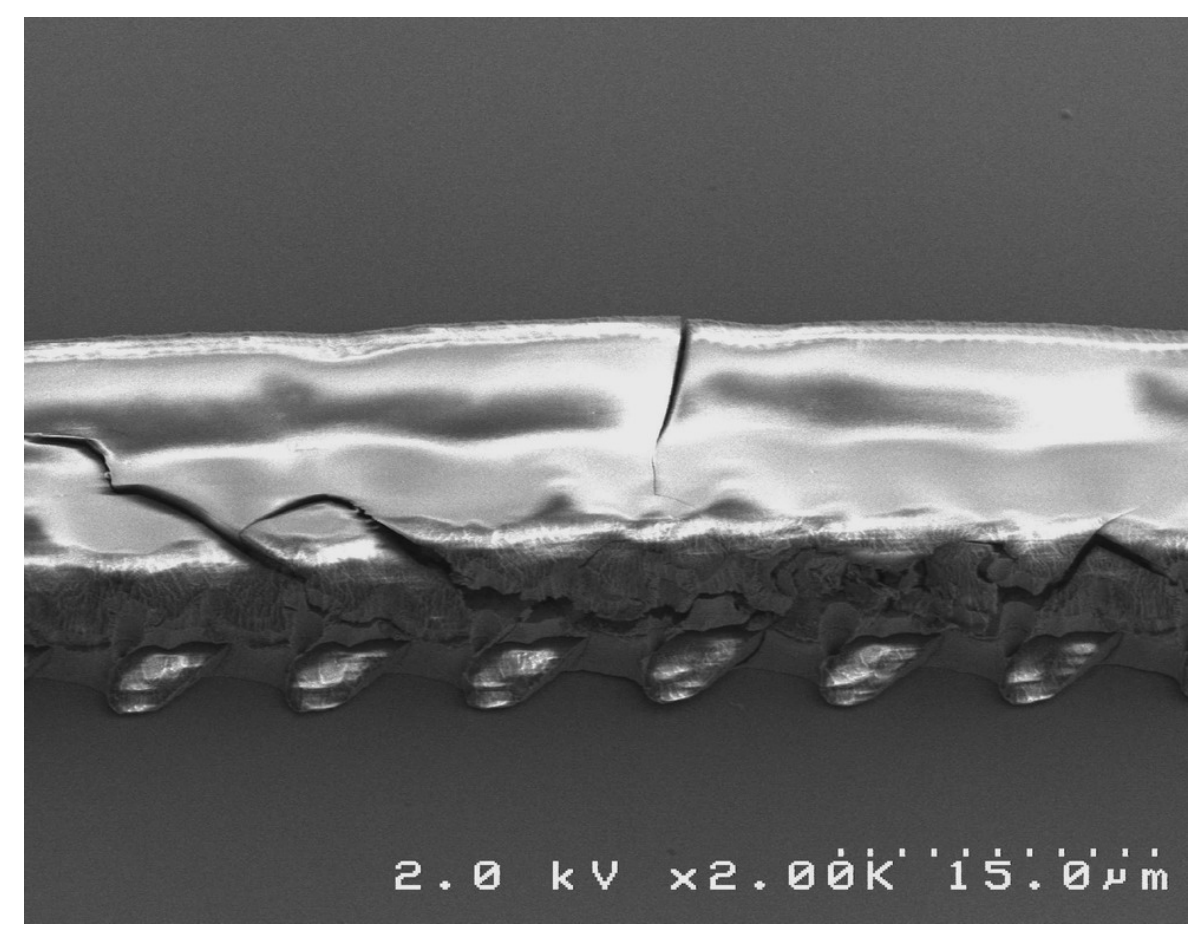

(a)

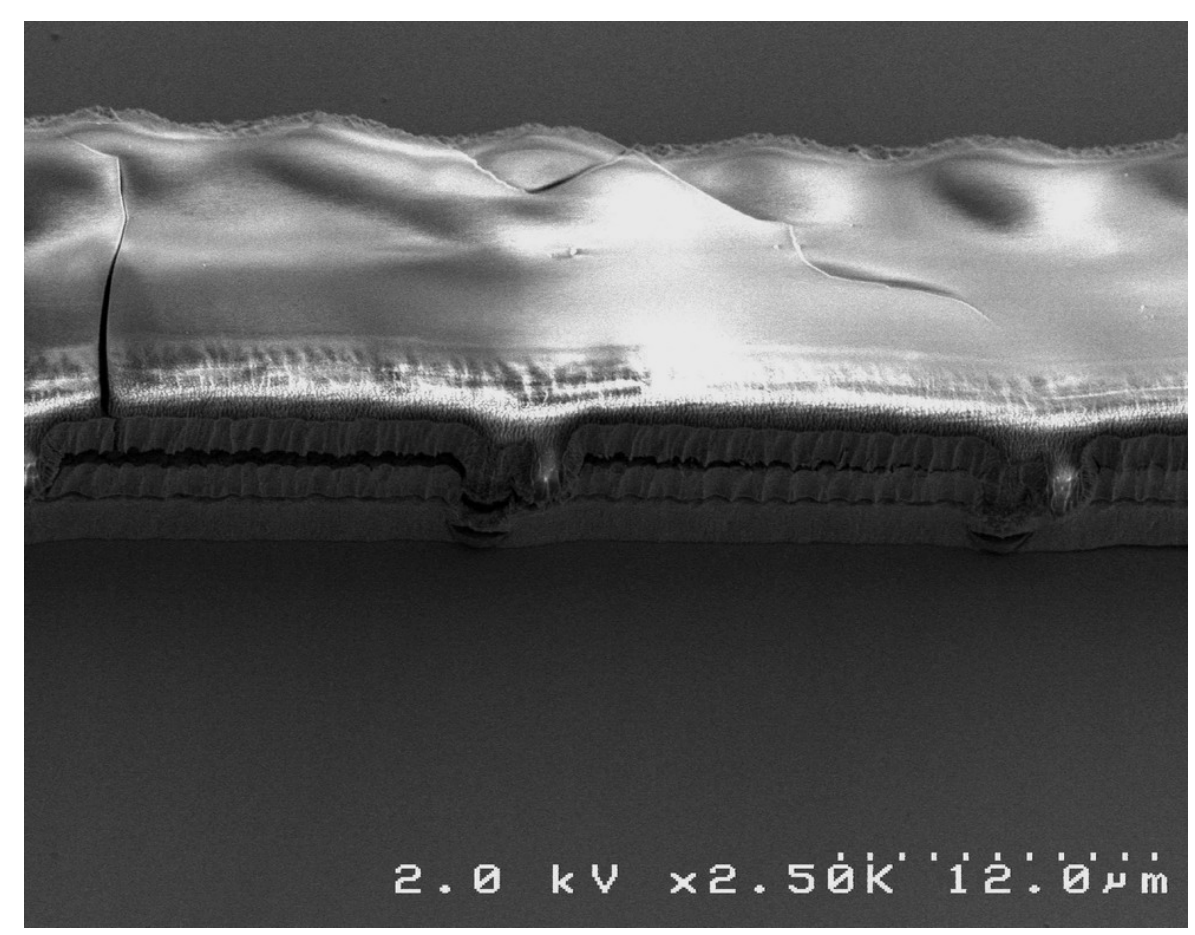

(b)

Figure 5.14 SEM images of the beam-d24r07c23 after the bending test. (a) Middle layer side; (b) Inner layer side. 
As seen in Figure 5.11, the initial yield is followed by a few rapid drops, which imply serious damage in the microstructure. Among them, some drops almost reach zero as seen in Figure 5.11-(10). In order to determine if these drops meant that the beams had been completely broken, SEM images were taken on both sides of the beams to check if through cracks exist in the microstructure.

Representative SEM images are shown in Figure 5.13 and Figure 5.14. Even though there are cracks across the top layer along the interface, and delamination between the top layer and the second layer, the beams are still partially connected.

Therefore, the rapid drops in the load-displacement curves do not represent catastrophic failure. As seen in Figure 5.13 and Figure 5.14, it is believed that the rapid drops are the responses to the appearance of the delamination. Large-scale delamination mainly happens between the top layer and the second layer. For most of the tested beams, the whole structures were still kept intact after the delamination happened and only two beams showed structural separation in the top layer. This provides the opportunity to observe the inside of the beams and helps to figure out what happened in the microstructures during the lateral testing.

Figure 5.15 shows a beam with part of its top layer broken away. It is believed that the crack initiated in the inner layer side and propagated in the top layer along the interface between the photoresist and the sputtered silicon. Once it reached the middle layer, its propagation was branched by the crossed arrangement of the trenches and formed zigzag traveling path. This clearly proves that the crossed arrangement of the trenches in the middle layer exerts its effect as the crack bridging mechanism. This image 
also proves that the beams did not fail catastrophically since the first layer was still intact and had strength to resist further load.
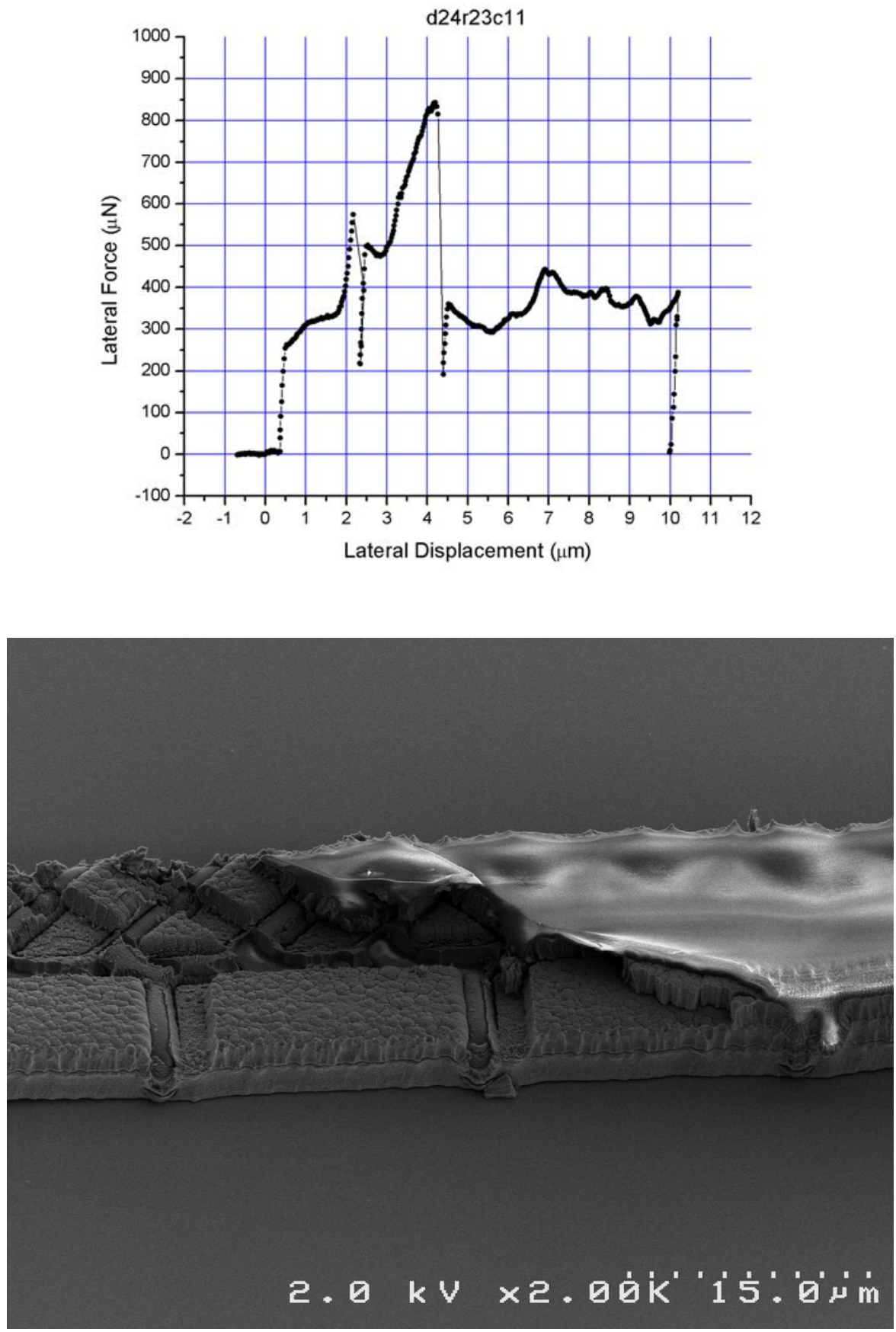

Figure 5.15 Beam-d24r23c11 with part of the top layer broken away and the corresponding load-displacement curve. 
Based on the evidence shown above, it is clear that the load-displacement curves shown in Figure 5.11 are not full description of the microstructure failure procedure.

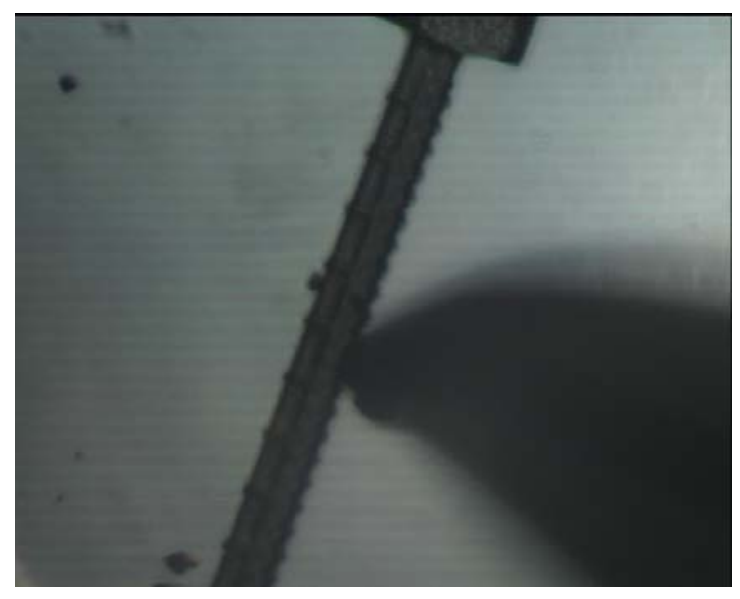

(a)

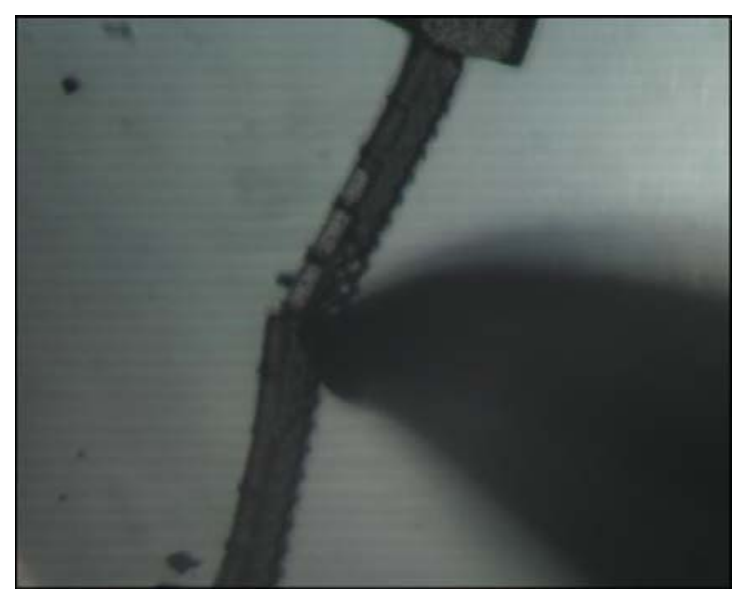

(b)

Figure 5.16 (a) Micro-probe position before pushing the beam. (b) Micro-probe position after a catastrophic failure.

In order to determine the ultimate displacement the beam can travel before a catastrophic failure occurs, a micro-probe was used to push the beam and the pushing procedure was videotaped. Figure 5.16 shows the micro-probe positions before pushing the beam and after generating a catastrophic failure. Using the beam width as a scalar 
$(20 \mu \mathrm{m})$, the failure displacement was estimated based on the traveling distance of the micro-probe. Six beams were tested and the average failure displacement was $16 \pm 1 \mu \mathrm{m}$.

\subsubsection{Measurements of toughening}

Based on the load-displacement curves presented in Figure 5.11, a straight line was drawn from the unloading point at $10 \mu \mathrm{m}$ displacement to the point with $16 \mu \mathrm{m}$ displacement and zero load to approximate the load-displacement curve in the displacement portion of $10 \mu \mathrm{m} \sim 16 \mu \mathrm{m}$. The schematic drawing is shown in Figure 5.17.

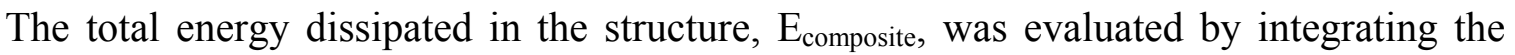
area under the load-displacement curve and the straight line. In Table 5.2, the third column lists the dissipated energy for the tested beams; the average dissipation of the composite is $\mathrm{E}_{\text {composite }}=6459 \pm 1544 \mu \mathrm{N} \bullet \mu \mathrm{m}$.

\section{d24r09c23}

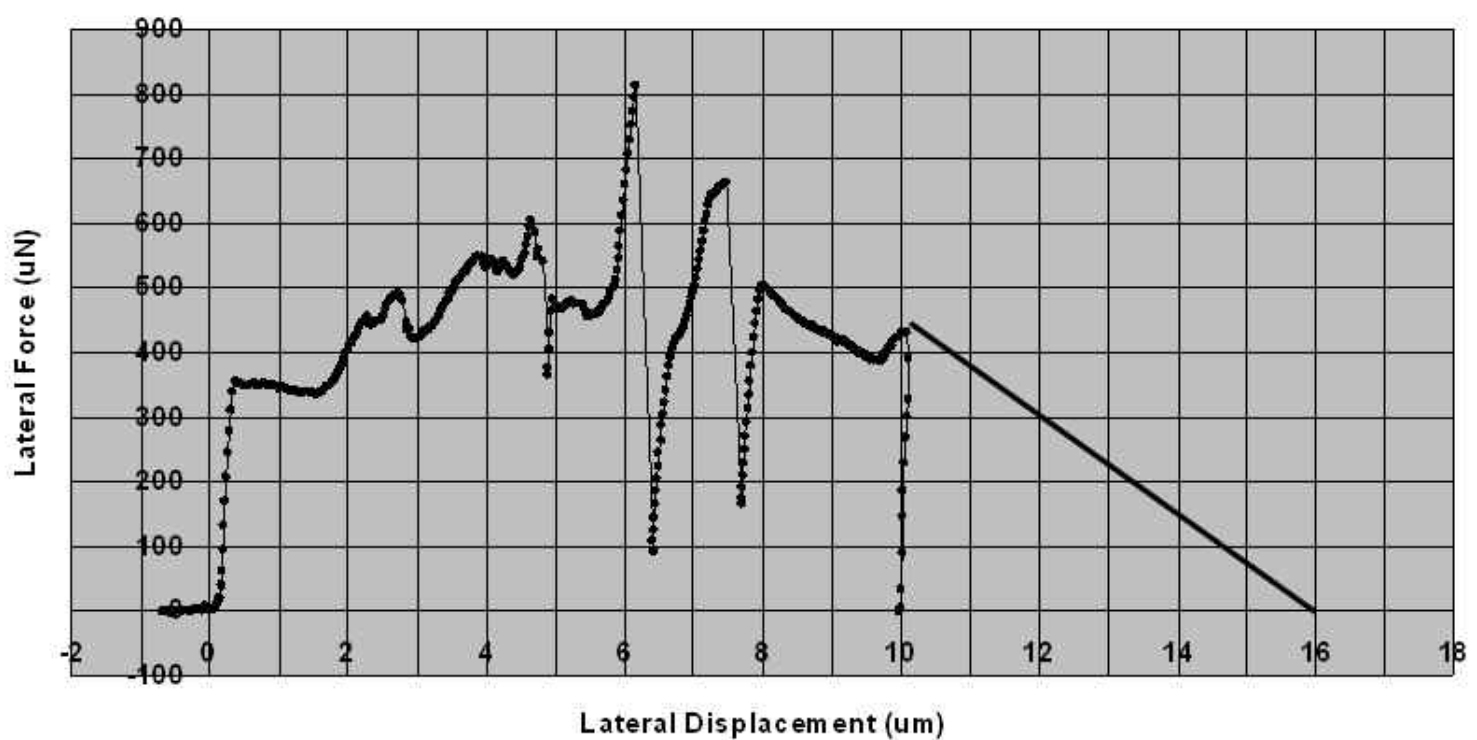

Figure 5.17 Schematic illustration of the extended load-displacement curve in the displacement portion of $10 \mu \mathrm{m} \sim 16 \mu \mathrm{m}$. 


\begin{tabular}{|c|c|c|c|c|}
\hline Label & $A_{E}(\mu \mathrm{N} \bullet \mu \mathrm{m})$ & $E_{\text {composite }}(\mu \mathrm{N} \bullet \mu \mathrm{m})$ & $A_{\text {postpeak }}(\mu \mathrm{N} \bullet \mu \mathrm{m})$ & $\mathrm{B}$ \\
\hline $\mathrm{d} 24 \mathrm{r} 09 \mathrm{c} 11$ & 61 & 6781 & 6720 & 0.009 \\
\hline $\mathrm{d} 24 \mathrm{r} 13 \mathrm{c} 11$ & 26 & 4745 & 4719 & 0.006 \\
\hline $\mathrm{d} 24 \mathrm{r} 05 \mathrm{c} 11$ & 60 & 8136 & 8076 & 0.007 \\
\hline $\mathrm{d} 24 \mathrm{r} 07 \mathrm{c} 23$ & 57 & 8753 & 8696 & 0.006 \\
\hline $\mathrm{d} 24 \mathrm{r} 09 \mathrm{c} 23$ & 51 & 5739 & 5688 & 0.009 \\
\hline $\mathrm{d} 24 \mathrm{r} 11 \mathrm{c} 23$ & 35 & 7805 & 7770 & 0.004 \\
\hline $\mathrm{d} 24 \mathrm{r} 13 \mathrm{c} 23$ & 73 & 8277 & 8204 & 0.009 \\
\hline $\mathrm{d} 24 \mathrm{r} 15 \mathrm{c} 23$ & 40 & 5210 & 5170 & 0.008 \\
\hline $\mathrm{d} 24 \mathrm{r} 21 \mathrm{c} 23$ & 40 & 5848 & 5808 & 0.007 \\
\hline $\mathrm{d} 24 \mathrm{r} 23 \mathrm{c} 11$ & 22 & 5165 & 5143 & 0.004 \\
\hline $\mathrm{d} 24 \mathrm{r} 19 \mathrm{c} 11$ & 43 & 4591 & 4548 & 0.009 \\
\hline MEAN & 46 & 6459 & 6413 & 0.007 \\
\hline STD & 16 & 1544 & 1534 & 0.002 \\
\hline
\end{tabular}

Table 5.2 Dissipated energy of the microstructures.

In order to compare the fracture resistance of the fabricated microstructure and the monolithic sputtered silicon, two measures of toughening were defined.

\section{Energy dissipation}

The toughness of the sputtered silicon, $2 \gamma$, was assumed to be $1 \mathrm{~N} / \mathrm{m}$. The dissipated energy of a monolithic sputtered silicon structure with the same dimensions as 
the fabricated microstructure, $\mathrm{E}_{\mathrm{Si}}$, was evaluated by multiplying the toughness by the area of the beam cross-section, which is $20 \mu \mathrm{m} \bullet 6.8 \mu \mathrm{m}$. Therefore, $\mathrm{E}_{\mathrm{Si}}$ is equal to $136 \mu \mathrm{N} \bullet \mu \mathrm{m}$. Thus, $\frac{E_{\text {composite }}}{E_{S i}}=\frac{6459}{136}=47$.

This result shows that the dissipated energy of the fabricated microstructure is almost two orders of magnitude higher than that of the monolithic sputtered silicon.

\section{Brittleness}

The structural brittleness is defined through the brittleness factor, $B=\frac{A_{E}}{A_{\text {postpeak }}}$, where $A_{E}$ is the area under the elastic part of the load-displacement curve (elastic strain energy), and $A_{\text {postpeak }}$ is the area in the post-peak region of the force-displacement curve. For monolithic sputtered silicon, the only energy dissipation is the energy of the newly created fracture surfaces, so that the $\mathrm{B}=\infty$.

For the fabricated microstructure, $\mathrm{B}$ is equal to $0.007 \pm 0.002$. This very small number implies that the fabricated microstructure exhibits significant ductility. 


\subsection{Conclusions}

\section{Innovative fabrication processes were designed and a 3-D micro-composite structure was fabricated.}

Multi-fabrication methods were employed to form the micro-composite structures using the innovative fabrication processes designed with respect to the available facilities and their process limits. Photoresist, which is normally used as etching mask, was employed as a structural component. This is the first attempt to use photoresist as a structural material. Due to the complexity of the fabrication, new fabrication recipes were developed to fulfill the specific tasks. A 3-D micro-composite structure with a crossedlamellar architecture was successfully fabricated. This fabricated microstructure realizes the designed goals and possesses the mechanisms of fracture resistance.

\section{Tunneling cracks appear in the inner layer and the designed crack bridging mechanism exerts its effect on blocking crack propagation.}

As seen in Figure 5.12-Figure 5.14, tunneling cracks appeared in the inner layer and continued to propagate along a zigzag path after they reached the middle layer, which includes the crossed-lamellar structure. The direct reflection of the non-straight crack path is the complex zigzag load-displacement curves shown in Figure 5.11. This proves that the designed microstructure possesses ductile and tough characters. In the load- 
displacement curve, slight drops represent the initialization of the tunneling cracks. The rapid drops are the responses to the longer crack propagation and the delamination.

\section{The dissipated energy of the fabricated microstructure is two-order higher than that of the monolithic material.}

The ratio of the dissipated energy of the fabricated microstructure and the monolithic sputtered silicon is 47 . This proves that the designed structure shows an increasing fracture resistance. The tunnel cracks in the inner layer, the crack branching along the layer interfaces and the crack bridging in the middle layer contribute to the relatively high energy dissipation. Since the designed microstructure has only two lengthscales, there is no doubt that higher order increase of the dissipated energy can be achieved with refined design to introduce more length-scales in the microstructure. The very small brittleness factor of the micro-composite structure proves that the designed structure possesses apparent ductility.

\subsection{Future works}

Based on the analysis described in the last section, the first requirement for achieving an even tougher structure is to exert constraint in the Z-direction so that no delamination can be easily initiated. This is can be fulfilled by sputtering the fourth silicon layer on the top of the three-layer composite structure. A 'final structure' photomask with wider features will be used to pattern the wafer. Therefore, the microstructure is completely covered by sputtered silicon in all directions. This silicon 
shell will keep the structure intact in Z-direction and improve the bending strength of the microstructure. Once the load reaches the bending strength of the silicon shell, tunnel cracking may happen in the designed inner layer. The crossed-lamellar structure in the designed middle layer will branch the tunneling cracks and help the microstructure to absorb more energy before it fails catastrophically. Much higher work of fracture is expected from this improvement.

Another possible attempt to improve the microstructure is to use $\mathrm{Si} / \mathrm{C}$ combination to replace current $\mathrm{Si} /$ Photoresist. Using $\mathrm{Si} / \mathrm{C}$ instead of $\mathrm{SiC} / \mathrm{C}$ discussed in Chapter 2 would have the advantage of the mature silicon fabrication processes. With this method, the bonding between the silicon and the carbon is expected to be strong and tight due to the nature of the carbon deposition methods, either sputtering or LPCVD. With the allowance of the budget, it could be attempted to deposit more than three layers in the Zdirection. This will further lower the ratio of the width over the depth and reduce the torsion effect that may be caused by the unexpected interaction between the indentation tip and the specimen sidewall.

In order to obtain a complete description of the structural failure procedure, a nanoindentation system with more than $10 \mu \mathrm{m}$ lateral traveling capability is preferred. An in-situ video system for real-time observation of the specimen deformation is definitely necessary. This will make it possible to match the particular structural deformations to the specific data points in the load-displacement curve and help to understand the mechanical behaviors of the microstructure better.

Overall, optimizing the micro-structural design, improving the fabrication process, and acquiring capable testing facility will make this project more successful. 


\section{Chapter Six}

\section{A Model for Strengthening of Polysilicon Devices}

\subsection{Introduction}

While the results presented in Kahn et al. ${ }^{[27]}$ clearly demonstrate that cyclic mechanical loading can either weaken or strengthen notched polysilicon MEMS structures, the irreversible deformation mechanisms responsible for such behavior have not yet been identified. Attempts to determine whether dislocations or microcracks are generated near the root of the notch under cyclic loads, using TEM, produced no conclusive evidence. This paper presents a model that predicts strengthening of notched polysilicon MEMS structures when a sufficiently large mean stress is followed by relatively small cyclic stress amplitude. Whether the same model could be extended to predict the weakening that results from the application of a large mean stress followed by large stress amplitude is yet to be determined.

To clarify what is meant by strengthening and weakening, the experimental procedure and results presented by Kahn et al. are reviewed. The loading applied to notched polysilicon MEMS structures involved four steps (Figure 6.1):

1. The loading is ramped up to produce a (tensile or compressive) mean stress, $\sigma_{\mathrm{m}}$, at the root of the notch.

2. $\sim 10^{5}-10^{6}$ cycles of stress amplitude, $\Delta \sigma$, is superimposed on $\sigma_{\mathrm{m}}$.

3. The loading is removed.

4. The loading is ramped up until the specimen fractures; the failure stress at the root of the notch is defined to be the strength. 
If the strength is higher (lower) than the average stress that produced fracture in specimens that were not subjected to steps 1 and 2, the polysilicon is said to have strengthened (weakened). As illustrated in Figure 6.2, when relatively small stress amplitudes are superimposed on relatively large tensile or compressive mean stresses, the polysilicon exhibits strengthening. On the contrary, larger stress amplitudes weaken polysilicon regardless of the value of mean stress (note that the devices could not be tested under large stress amplitudes superimposed on large tensile mean stresses). The representative strengthening results corresponding to a $2.0 \mathrm{GPa}$ stress amplitude are shown in Figure 6.3. The symmetry of the data with respect to the sign of the mean stress suggests a shear-dominated strengthening mechanism such as plasticity.

\subsection{Plastic Flow in Amorphous Silicon and the Drucker-Prager}

\section{Model}

A possible explanation for the strengthening is that the cyclic loading leads to some sort of plastic deformation near the root of the notch, and results in residual compressive stresses upon unloading. The compressive prestress would result in a higher apparent strength when the structure is subsequently loaded in monotonically increasing tension. This possibility is supported by the molecular dynamics calculations performed by Demkowicz and Argon ${ }^{[29]}$, which show that amorphous silicon can plastically deform. If the grain boundaries in Kahn et al.'s polycrystalline silicon MEMS structures were in fact comprised of amorphous silicon, then under certain levels of mean stress and stress amplitude, plasticity could have evolved along the grain boundaries, and compressive residual stresses could have developed at the root of the notch upon removal of the loads. 
The molecular dynamics simulations involved shear loading at constant volume. The system pressure, deviatoric stress, and deviatoric plastic strain are defined, respectively, as

$$
\begin{gathered}
p=-\frac{\sigma_{i i}}{3} \\
s_{i j}=\sigma_{i j}-\frac{\sigma_{i i}}{3} \delta_{i j} \\
e_{i j}^{p}=\varepsilon_{i j}^{p}-\frac{\varepsilon_{i i}^{p}}{3} \delta_{i j}
\end{gathered}
$$

where the $\sigma_{i j}\left(\varepsilon_{i j}^{p}\right)$ are the components of the stress (plastic strain) tensor, and repeated indices are summed.

Using the Stillinger-Weber empirical potential for silicon ${ }^{[30]}$, Demkowicz and Argon created amorphous samples with four different initial densities by melting the diamond cubic crystal structure, and then slowly quenching the liquid at different rates using constant pressure molecular dynamics.

For four values of initial density, $\rho$, the deviatoric stress and pressure as functions of deviatoric strain are shown in Figure 4. The plasticity is very sensitive to the density of the initial unstressed amorphous silicon, and can produce either dilatancy or compaction (the pressure in the constant volume simulations increases or decreases). For the lowest density, elastic loading terminates with a sharp yield phenomenon that is followed by significant strain softening and concomitant large drop in system pressure. As pointed out by Demkowicz and Argon, the pressure drop, which implies compaction in a constant pressure simulation, is opposite to the behavior of metallic glasses, which expand during deformation. For the two highest densities, the plastic deformation is 
associated with an increase in pressure, implying that under constant pressure the silicon would dilate. The plastic deformation predicted by the atomistic calculations can be approximated using the Drucker-Prager plasticity model. The yield surface (function) is defined as

$$
F=\sigma_{e}-p \tan \beta-c=0
$$

where $\sigma_{e}=\sqrt{\frac{3}{2} s_{i j} S_{i j}}$ is the equivalent (von Mises) stress, $\beta$ is the friction angle, and $c=\sqrt{3} \tau_{o}$ is the cohesion of the material written in terms of the shear strength $\tau_{o}$. A schematic of the yield surface for the model is shown in Figure 6.5.

The plastic (flow) potential is

$$
G=\sigma_{e}-p \tan \psi
$$

where $\psi$ is the dilatation angle. The increments of plastic strain are obtained from the potential as

$$
d \varepsilon_{i j}^{p}=d \lambda \frac{\partial G}{\partial \sigma_{i j}}
$$

where

$$
d \lambda=\frac{d \varepsilon^{p}}{1-\frac{1}{3} \tan \psi}
$$

and $d \varepsilon^{p}=\sqrt{\frac{2}{3} d \varepsilon_{i j}^{p} d \varepsilon_{i j}^{p}}$ is the equivalent plastic strain increment. 
The curves in Figure 6.4 were used as inputs into the finite element program ABAQUS ${ }^{[31]}$ to calibrate values of friction angle, $\beta$, dilatation angle $\psi$, and relevant isotropic hardening parameters, using a two dimensional plate, comprised of four-node quadrilateral elements, subjected to a shear deformation. The elastic modulus of the amorphous silicon was obtained as 150GPa from the data in Figure 6.4(a), and the Poisson's ratio was assumed to be 0.22 . The representative trial curves for initial density $\rho=2342 \mathrm{~kg} / \mathrm{m}^{3}$ are shown in Figure 6.6, the best fit being provided by the combination $\beta=8.5^{\circ}, \psi=-35^{\circ}$. Because the friction and dilatation angles are not equal, the plasticity model is nonassociated, and the material stiffness matrices are not symmetric.

As explained subsequently, the strengthening is predicted by both dilating and contracting amorphous grain boundaries. The demonstrative simulations presented next were performed using the Drucker-Prager parameters corresponding to the lowest density silicon.

\subsection{Simulations of Cyclic Loading on Notched Polysilicon Specimens}

The plasticity model was used to simulate cyclic loading experiments on the notched specimen shown in Figure 6.7. This geometry is representative of the specimens tested by Kahn et al. The stresses near the root of the notch are controlled by the applied traction, $\sigma$. To determine whether the level of stresses applied in Kahn et al.'s experiments could produce plastic deformation within the amorphous grain boundaries near the root of the notch, two finite element models were used. The first, shown in 
Figure 6.8 , is a local-global model that retains a discrete description of the crystalline structure surrounding the notch, while efficiently incorporating the far-field behavior through a homogenized polysilicon with a Young's modulus equal to $160 \mathrm{GPa}$ and a Poisson's ration equal to 0.22 . The randomly sized crystals, generated through PoissonVoronoi tessellation ${ }^{[32]}$, have linear dimensions in the range $\sim 200-300 \mathrm{~nm}$, and are separated by $\sim 1-2 \mathrm{~nm}$ thick grain boundaries made of amorphous silicon. The second model, shown in Figure 6.10 and discussed subsequently, consists of homogeneous silicon containing a single plastically deforming grain boundary. It is noted that the first finite element model does not include the three dimensional geometry of the fine grained polysilicon tested by Kahn et al. Instead, it is a demonstrative plane stress model with columnar grains.

With respect to the loading described in Figure 6.1, the failure map shown in Figure 6.2 is explored by applying 500 cycles to each of four combinations of mean stress and stress amplitude; (1) $\sigma_{\mathrm{m}}=0.8 \mathrm{GPa}, \Delta \sigma=1.6 \mathrm{GPa}$ (small tensile mean stress, small stress amplitude), (2) $\sigma_{\mathrm{m}}=-1.0 \mathrm{GPa}, \Delta \sigma=1.6 \mathrm{GPa}$ (small compressive mean stress, small stress amplitude), (3) $\sigma_{\mathrm{m}}=2.0 \mathrm{GPa}, \Delta \sigma=2.0 \mathrm{GPa}$ (large tensile mean stress, small stress amplitude), (4) $\sigma_{\mathrm{m}}=-3.5 \mathrm{GPa}, \Delta \sigma=2.0 \mathrm{GPa}$ (large compressive mean stress, small stress amplitude).

In agreement with the experimental results, the structure remained elastic for cases (1) and (2); no residual stresses developed upon removal of the cyclic loading. Therefore no strengthening would be observed upon subsequent tensile loading.

Figure 6.9 shows the distributions of the stress component $\sigma_{22}$ near the root of the notch for cases (3) and (4). It is observed that after 500 cycles modest levels of 
compressive stresses develop; $\sim 0.5-1 \mathrm{GPa}$ for the tensile mean stress and $\sim 0.1-0.3 \mathrm{GPa}$ for the compressive mean stress. However, it was observed during the finite element simulations that the magnitude of the compressive residual stresses increases with increasing number of cycles, the rate of increase being larger for the case of tensile mean stress.

The detailed crystalline description precludes the possibility of simulating a larger number of load cycles in the nonlinear material structural model. Therefore, taking advantage of the fact that the local-global simulations indicated that plastic deformation develops in only one grain boundary, the model shown in Figure 6.10 was constructed to simulate larger numbers of cycles. This model, involving one grain boundary $216 \mathrm{~nm}$ long near the root of the notch, was loaded for 1000 cycles with the aforementioned combinations of mean stress and stress amplitude.

As for the local-global model, no residual stresses develop for the first two cases. Figure 6.11 shows the results for $\sigma_{\mathrm{m}}=2.0 \mathrm{GPa}, \Delta \sigma=2.0 \mathrm{GPa}$ and $\sigma_{\mathrm{m}}=-3.5 \mathrm{GPa}, \Delta \sigma$ $=2.0 \mathrm{GPa}$. Both cases produce significant residual compressive stresses; $\sim 1.39 \mathrm{GPa}$ maximum compression for the case of tensile mean stress, and $\sim 0.9 \mathrm{GPa}$ maximum compression for the case of compressive mean stress. Remarkably, the levels of compressive stress are of the same order as the experimentally observed strengthening.

To determine whether the predictions are sensitive to the dilatant/compactive nature of the plastic deformation, the simulations were repeated with all parameters kept the same except for the dilatancy angle, which was assumed as $\psi=+35^{\circ}$ (the sign was switched to produce dilatancy). Figure 6.12 shows that the residual stress distribution is 
similar to that predicted by the previous model, the largest value of residual compressive stress being slightly higher.

\subsection{Conclusions}

The model presented in this paper predicts levels of cyclic loading-induced strengthening of polysilicon MEMS structures that are qualitatively and quantitatively consistent with experimental data. The apparent strengthening results from the residual compressive stresses that result from the plastic deformation of amorphous grain boundaries. Because the arrangement of the grains and grain boundaries is random, the demonstrative simulations presented in this paper represent but discrete realizations of a stochastic process. An ensemble of such simulations could be calculated using a Monte Carlo procedure to quantify the statistical nature of the strengthening. 


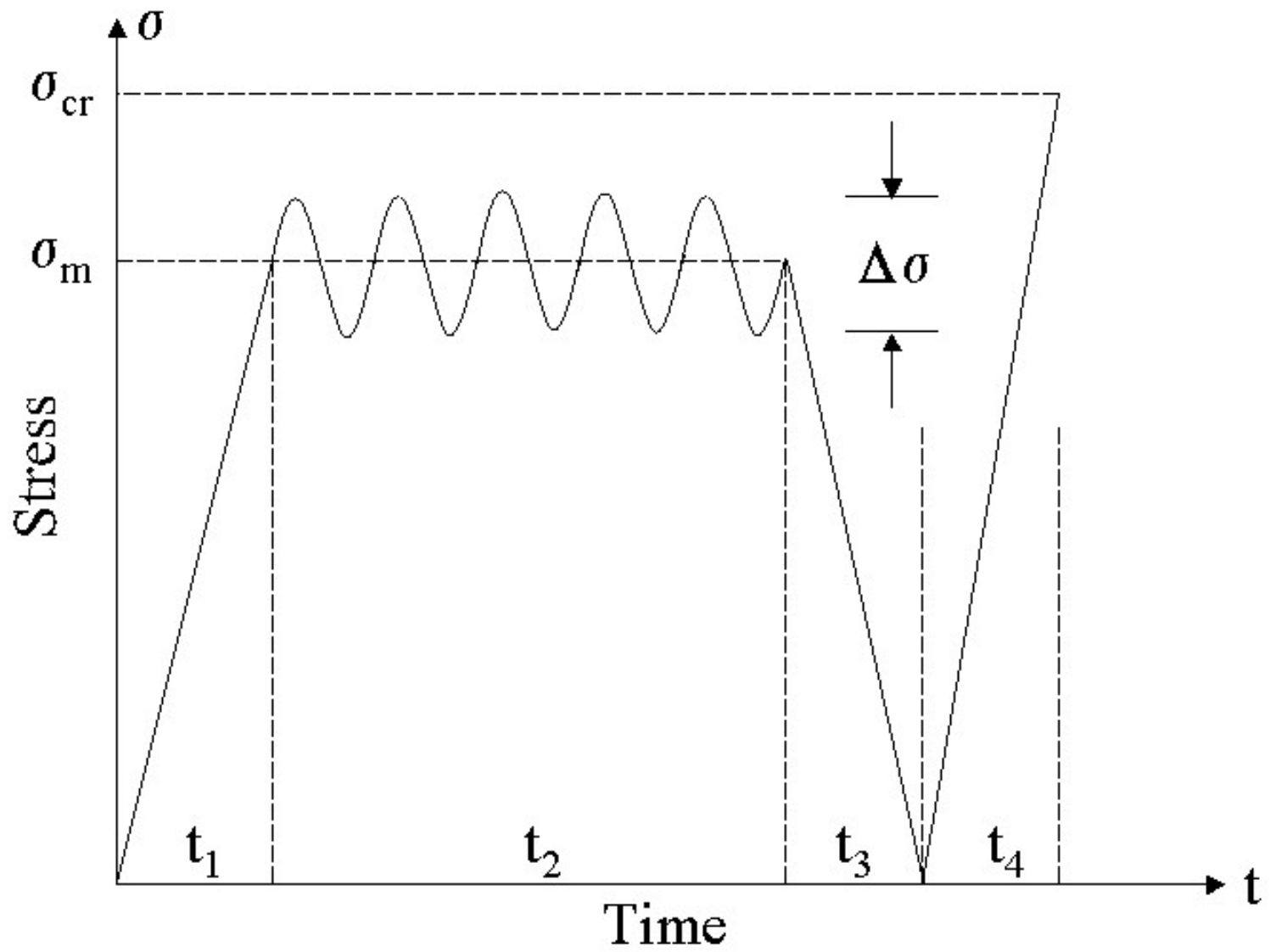

Figure 6.1 Schematic illustration of cyclic loading applied to notched polysilicon MEMS specimens. 


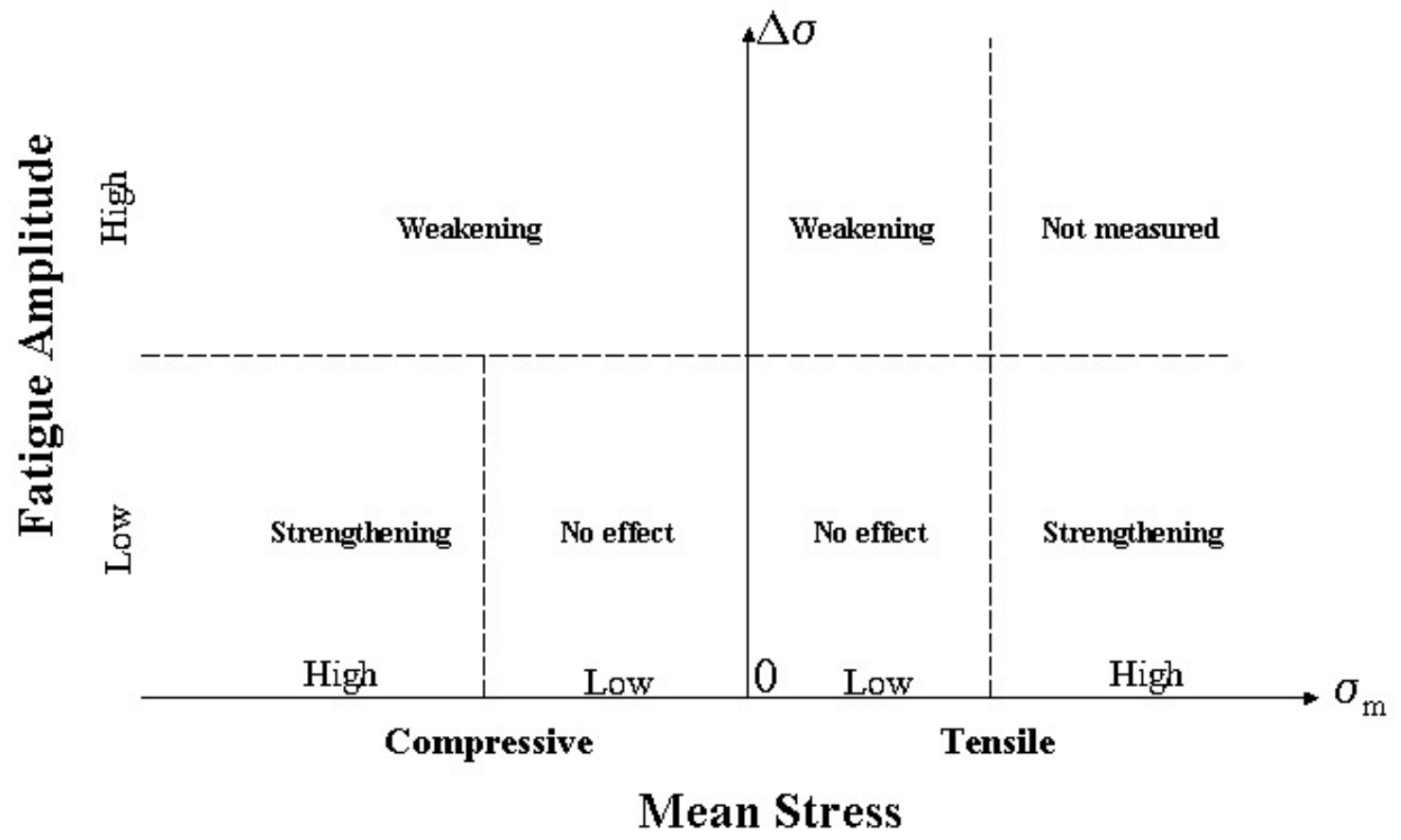

Figure 6.2 Effects of mean stress and stress amplitude on nominal strength of notched polysilicon MEMS structures. 


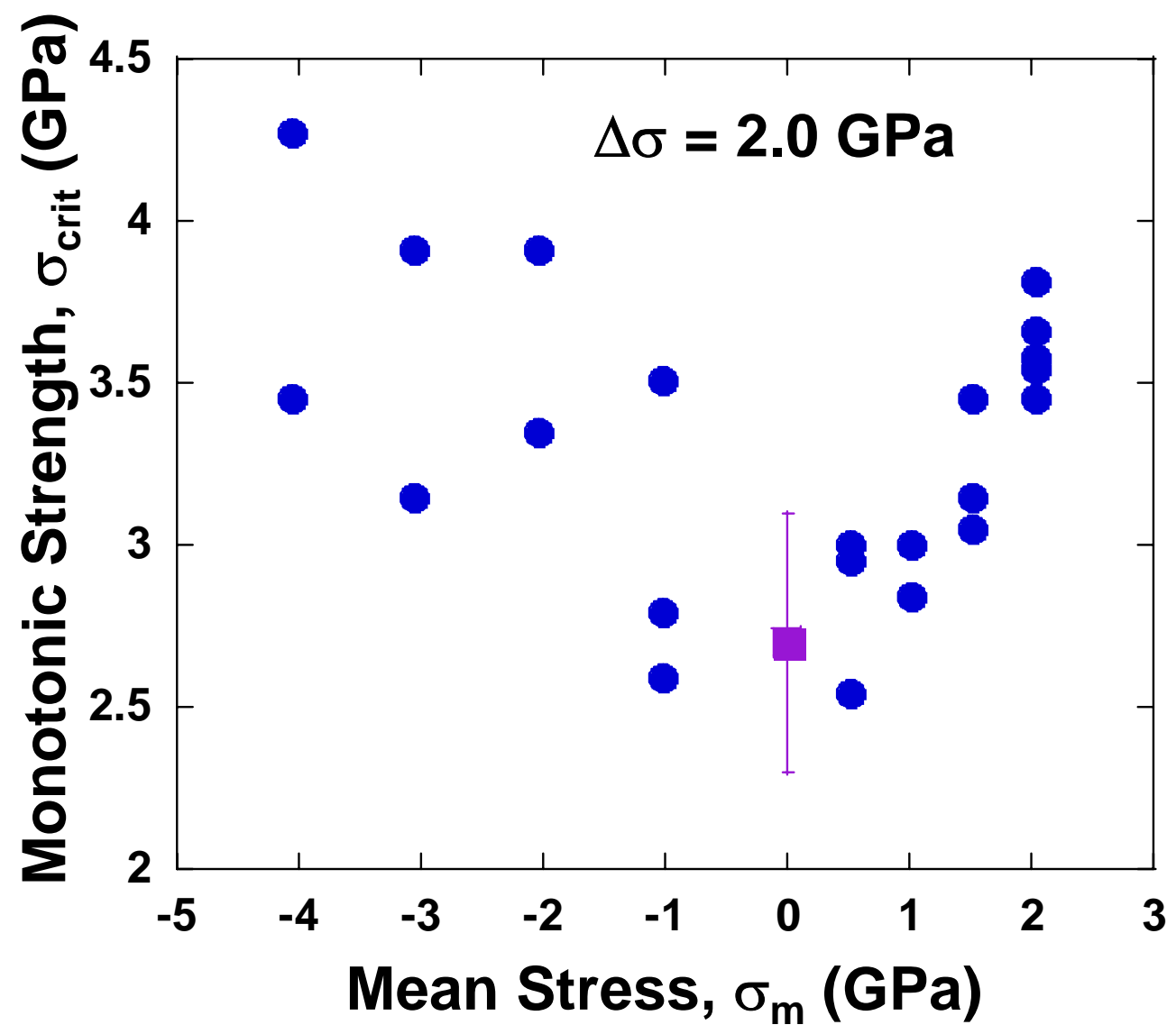

Figure 6.3 Effects of mean stress on nominal strength of notched polysilicon MEMS structures for a relatively low value of cyclic stress amplitude, $\Delta \sigma=2 \mathrm{GPa}$. 


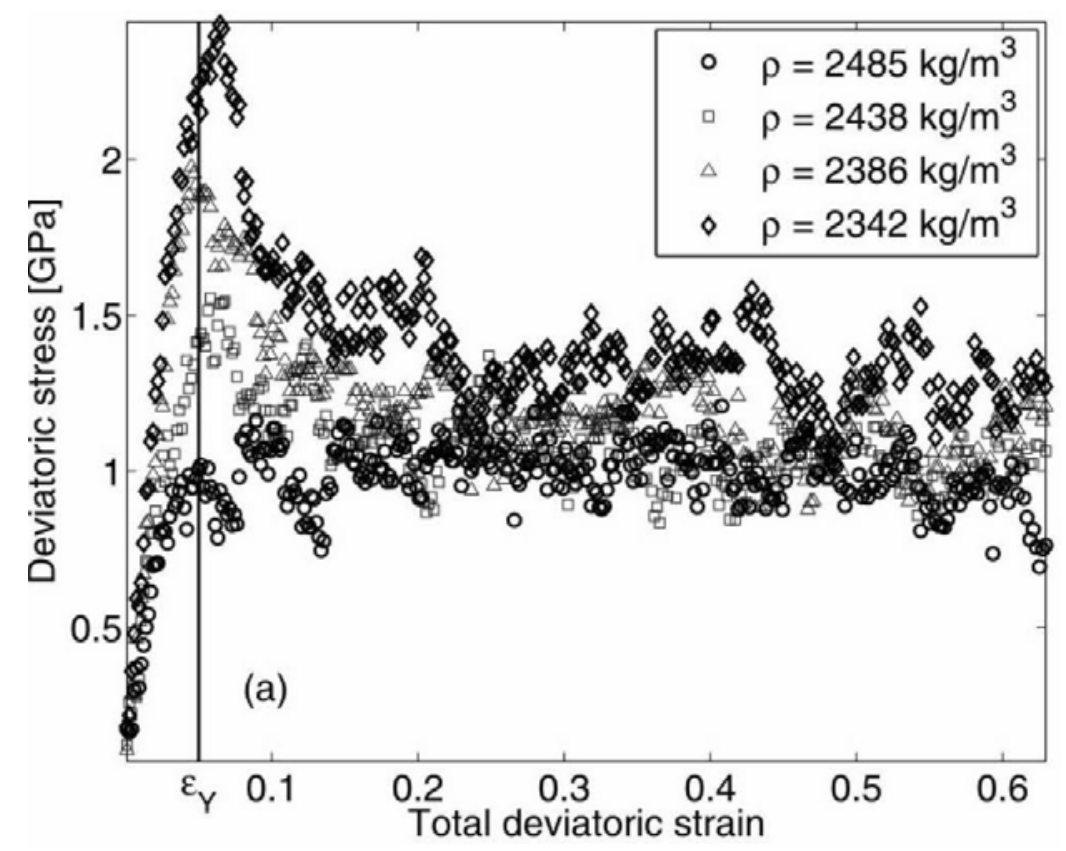

(a)

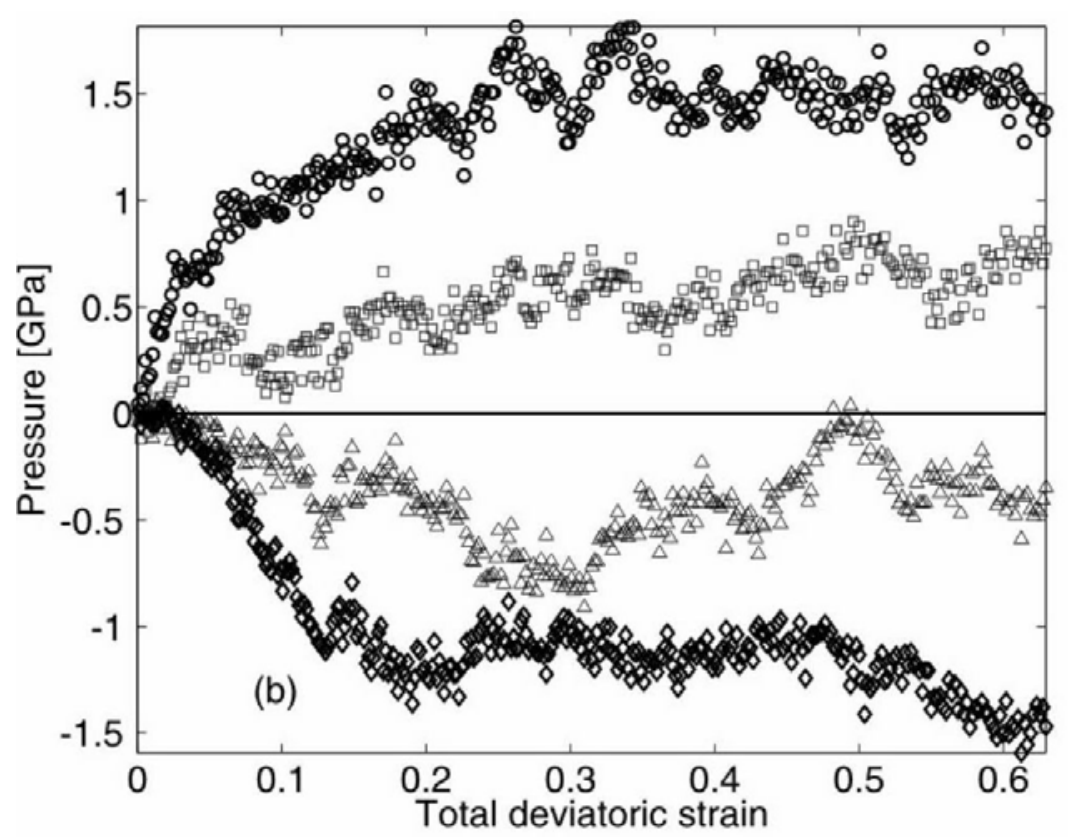

(b)

Figure 6.4 Plastic-like behavior of amorphous silicon predicted by atomistic calculations; (a) Deviatoric stress versus deviatoric strain; (b) Pressure versus deviatoric strain. 


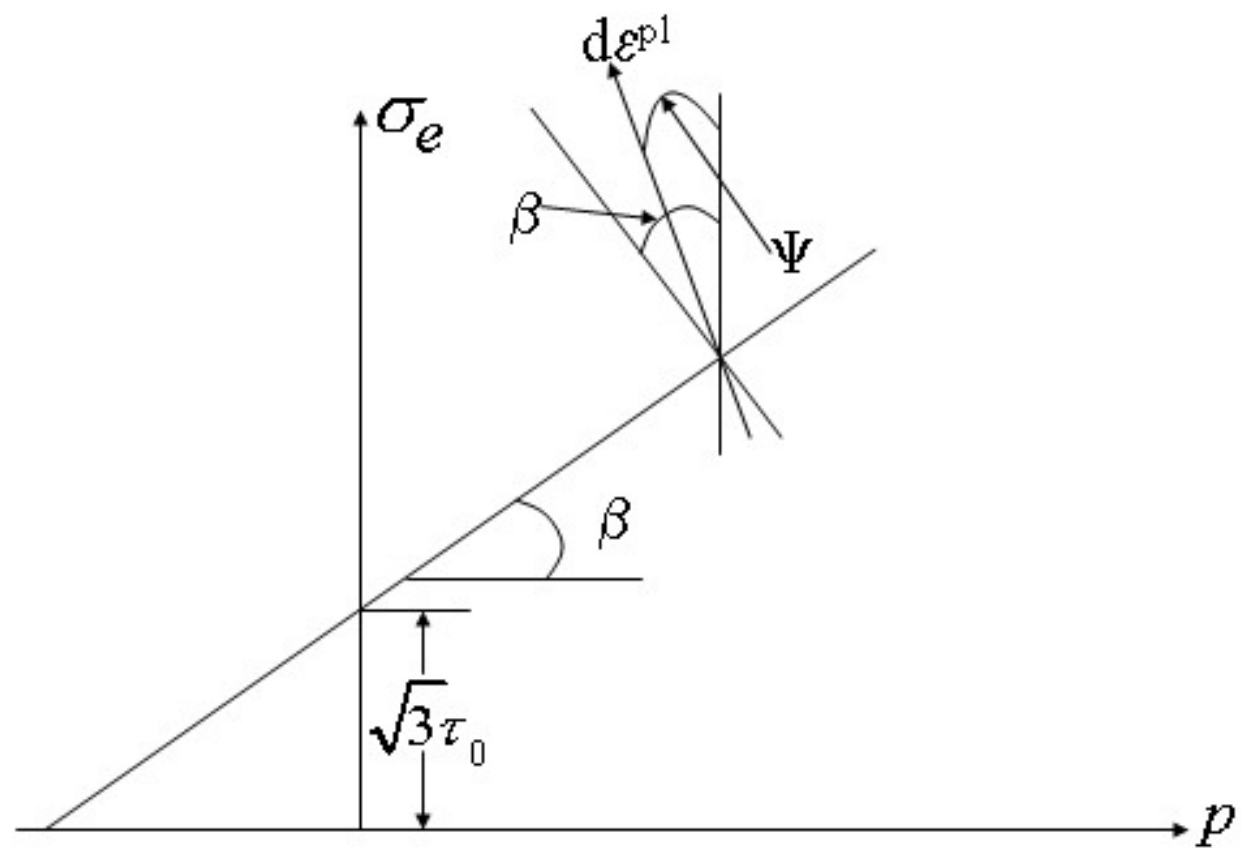

Figure 6.5 Yield surface of linear Drucker-Prager plasticity model. 


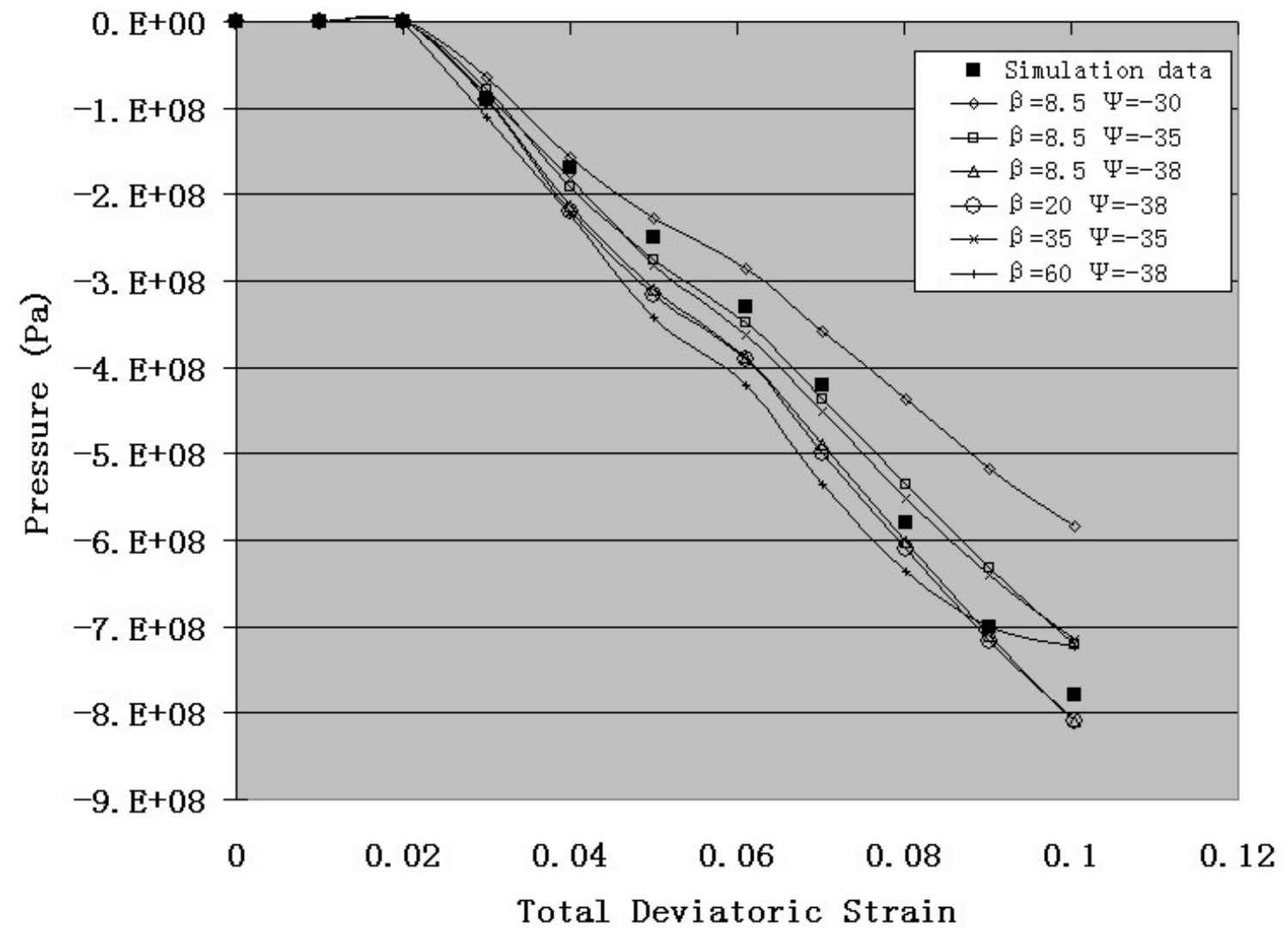

Figure 6.6 Calibration of friction and dilatation angles of linear Drucker-Prager model for amorphous silicon with initial density $\rho=2342 \mathrm{~kg} / \mathrm{m} 3$. 


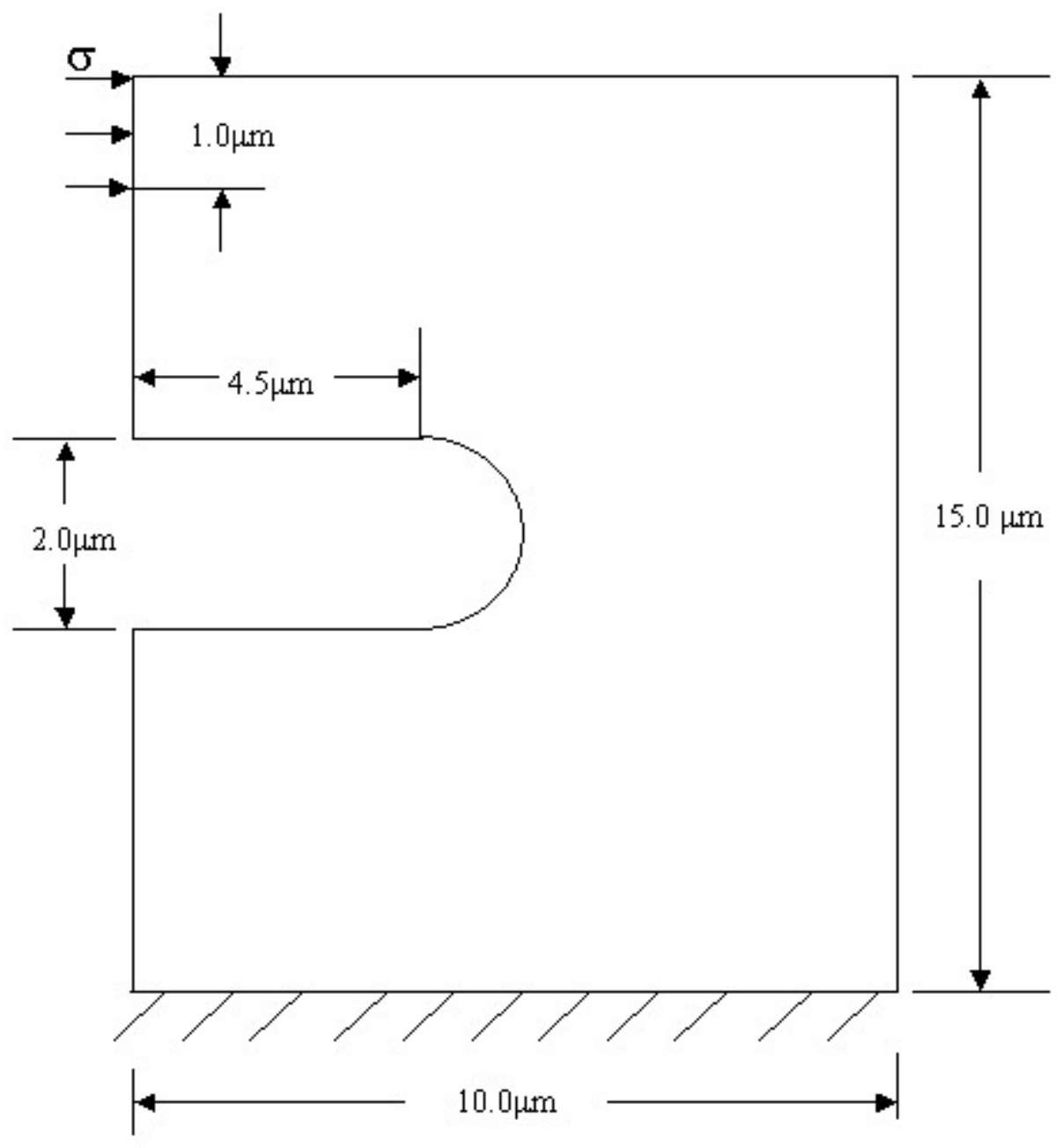

Figure 6.7 Geometry of notched polysilicon specimen subjected to cyclic loading. 


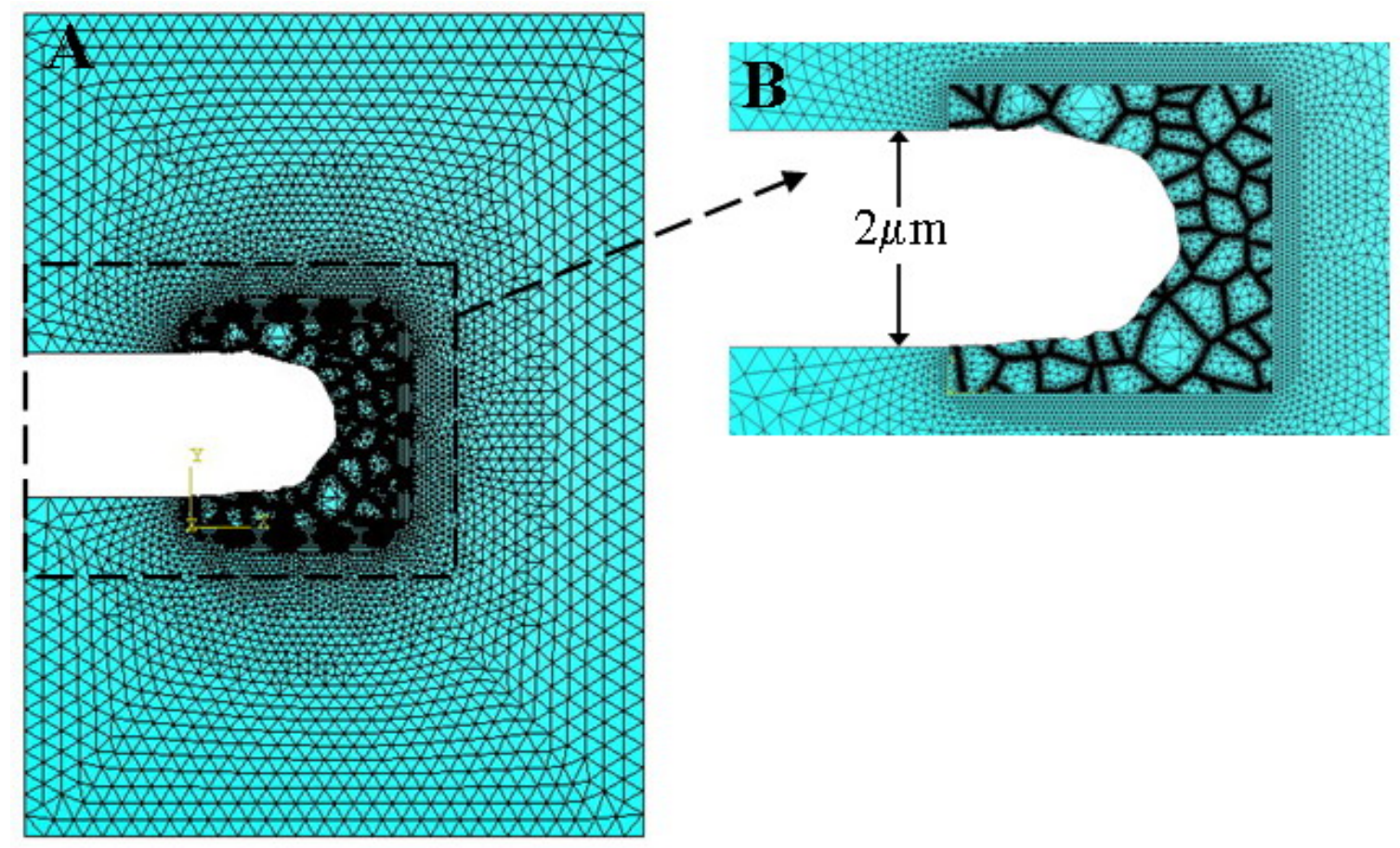

Figure 6.8 (A) Local-global model of a notched polysilicon MEMS specimen. (B) Closeup view of the root of the notch, showing the discrete polycrystalline structure surrounded by homogeneous silicon. 


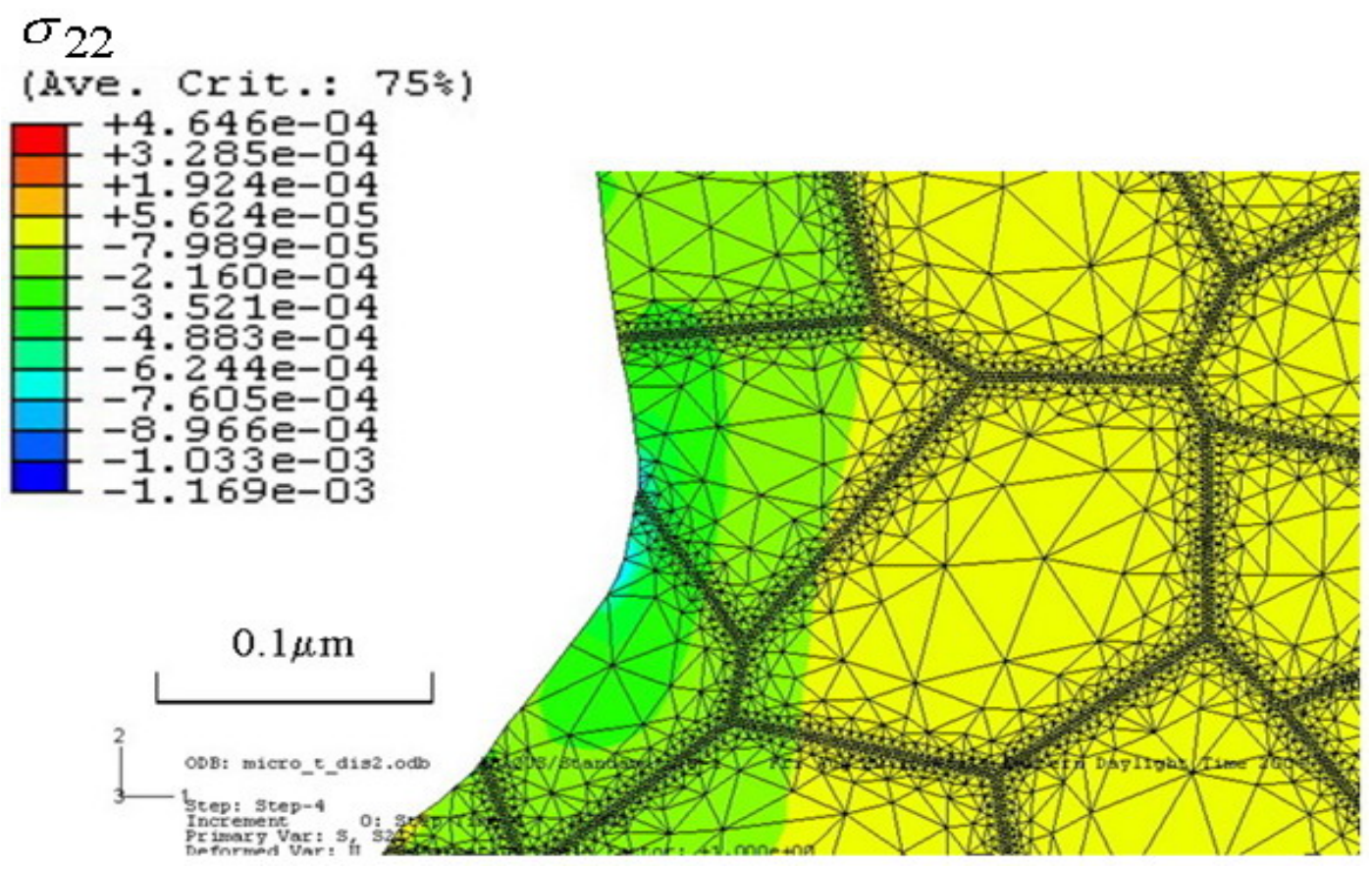

(a)

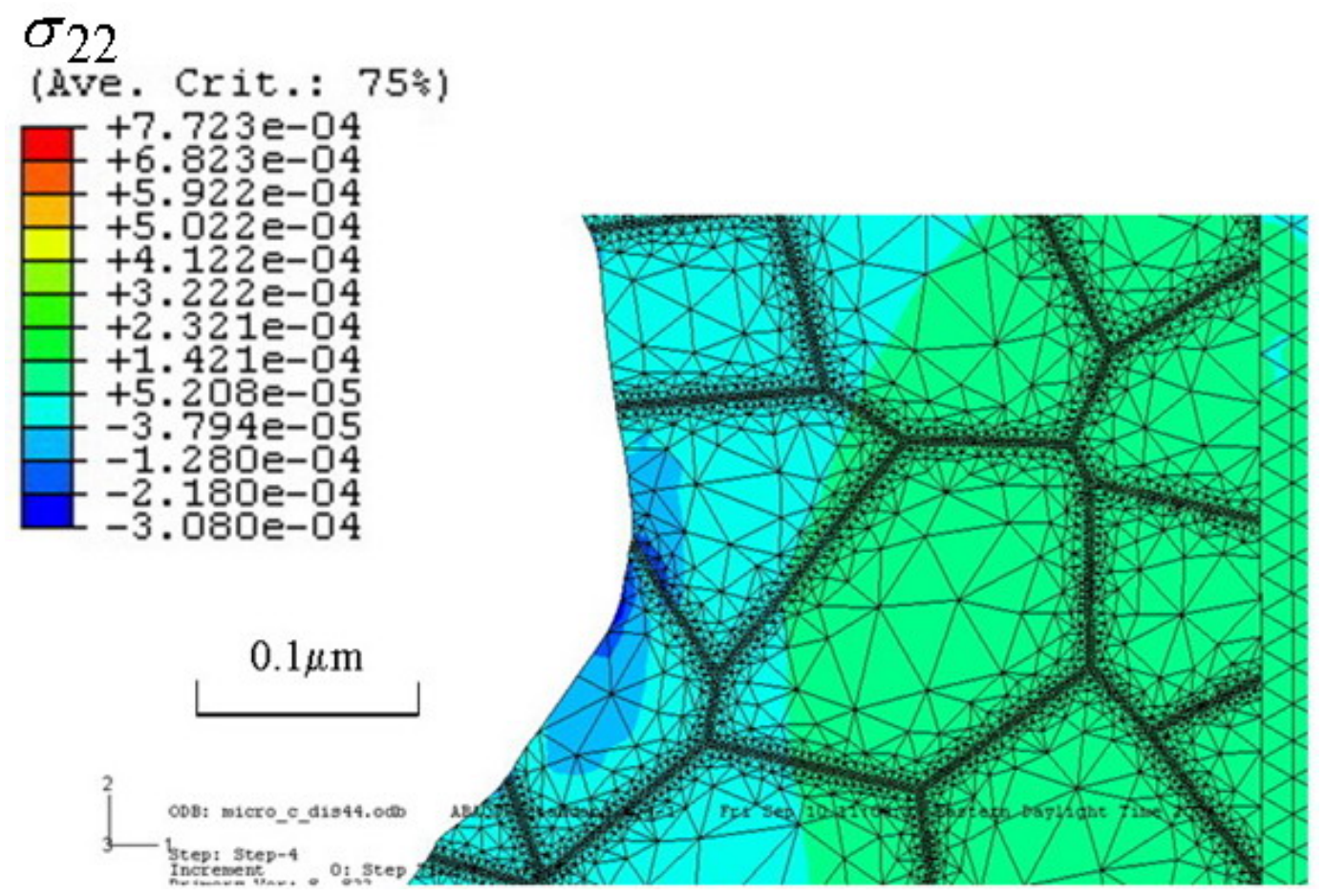

$75 \div)$

(b)

Figure 6.9 Residual stress distribution after 500 cycles; (a) $\sigma_{\mathrm{m}}=2.0 \mathrm{GPa}, \Delta \sigma=2.0 \mathrm{GPa}$, (b) $\sigma_{\mathrm{m}}=-3.5 \mathrm{GPa}, \Delta \sigma=2.0 \mathrm{GPa}$. 


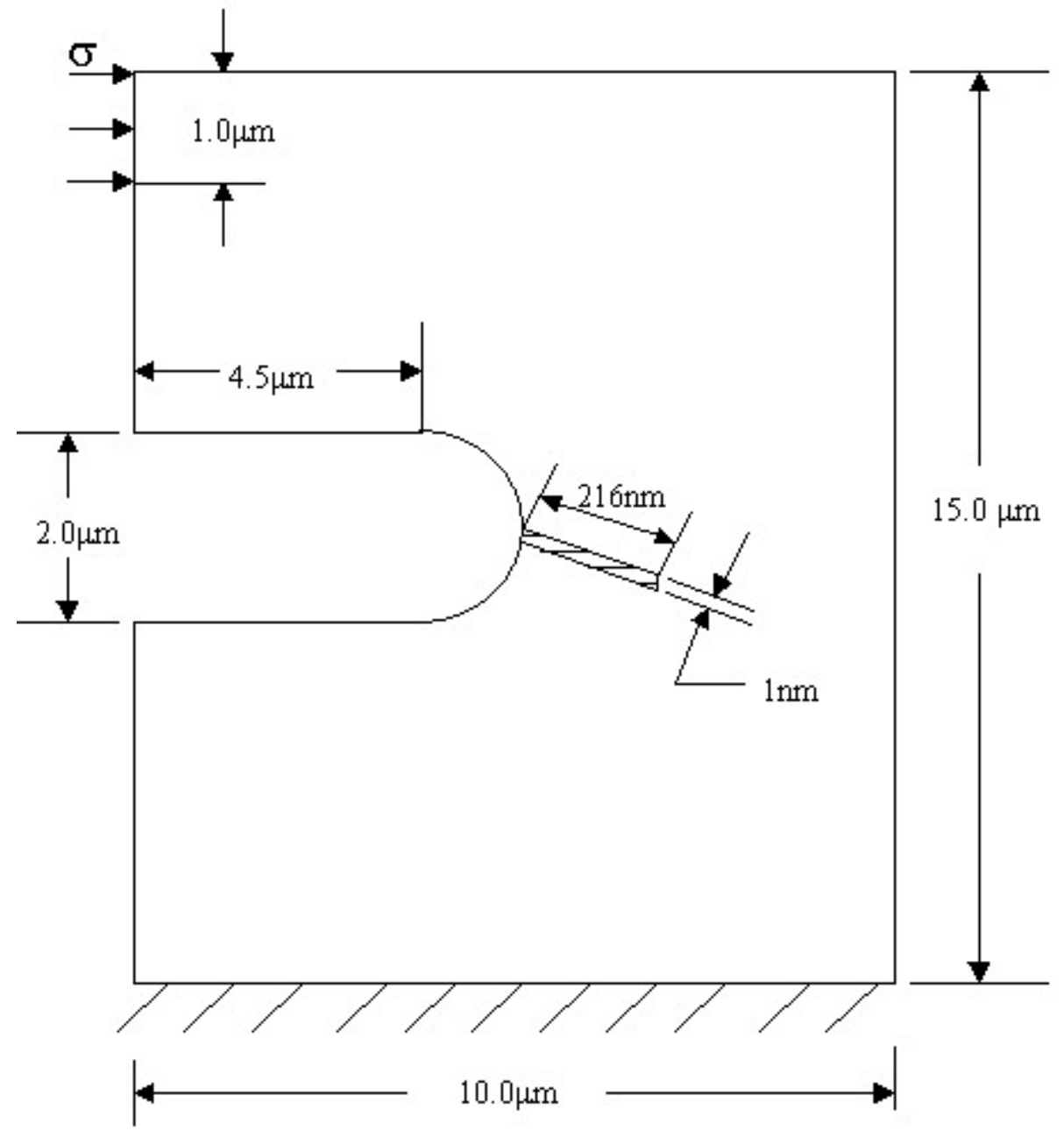

Figure 6.10 Homogeneous notched polycrystalline structure containing a single plastically deforming grain boundary. 


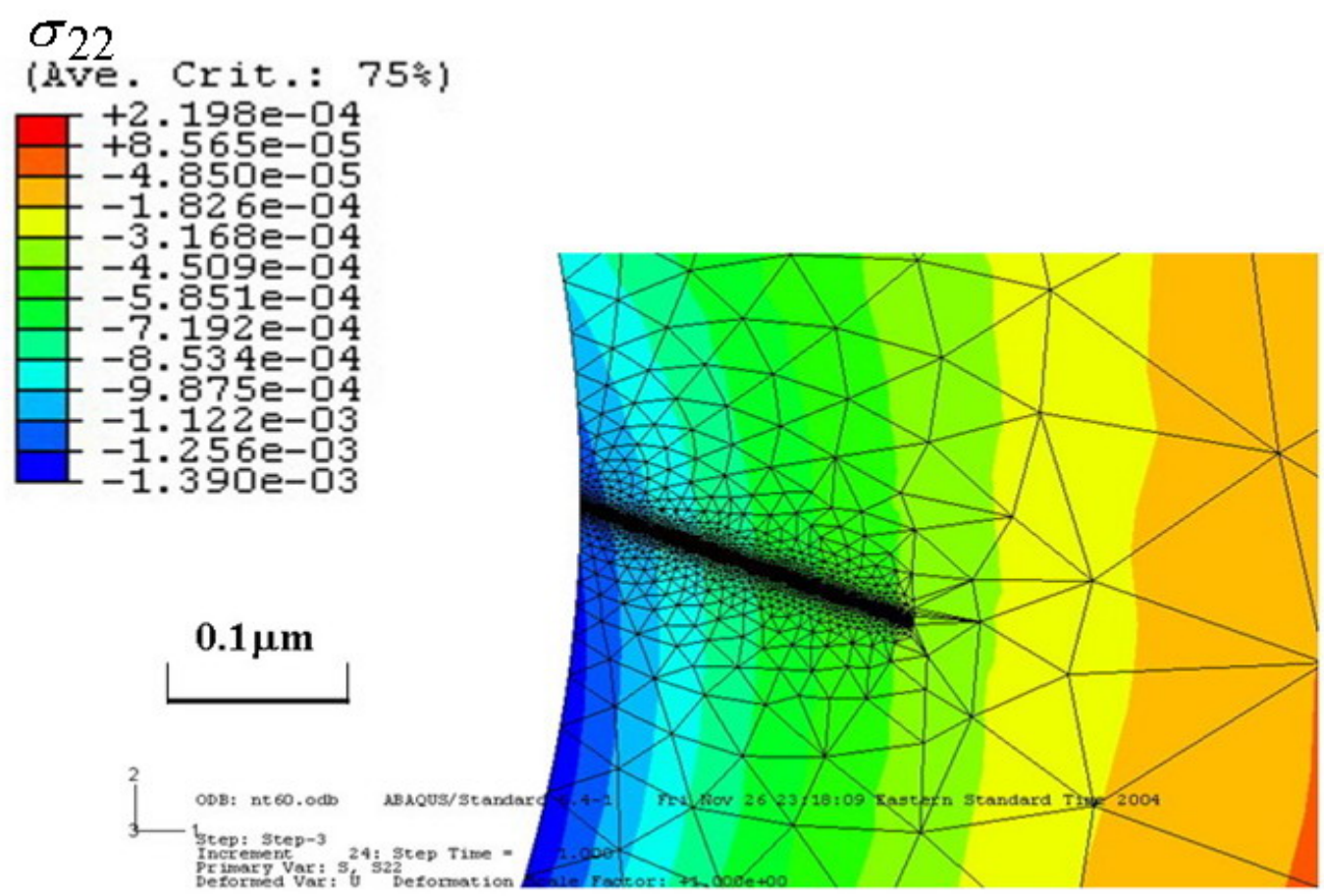

(a)
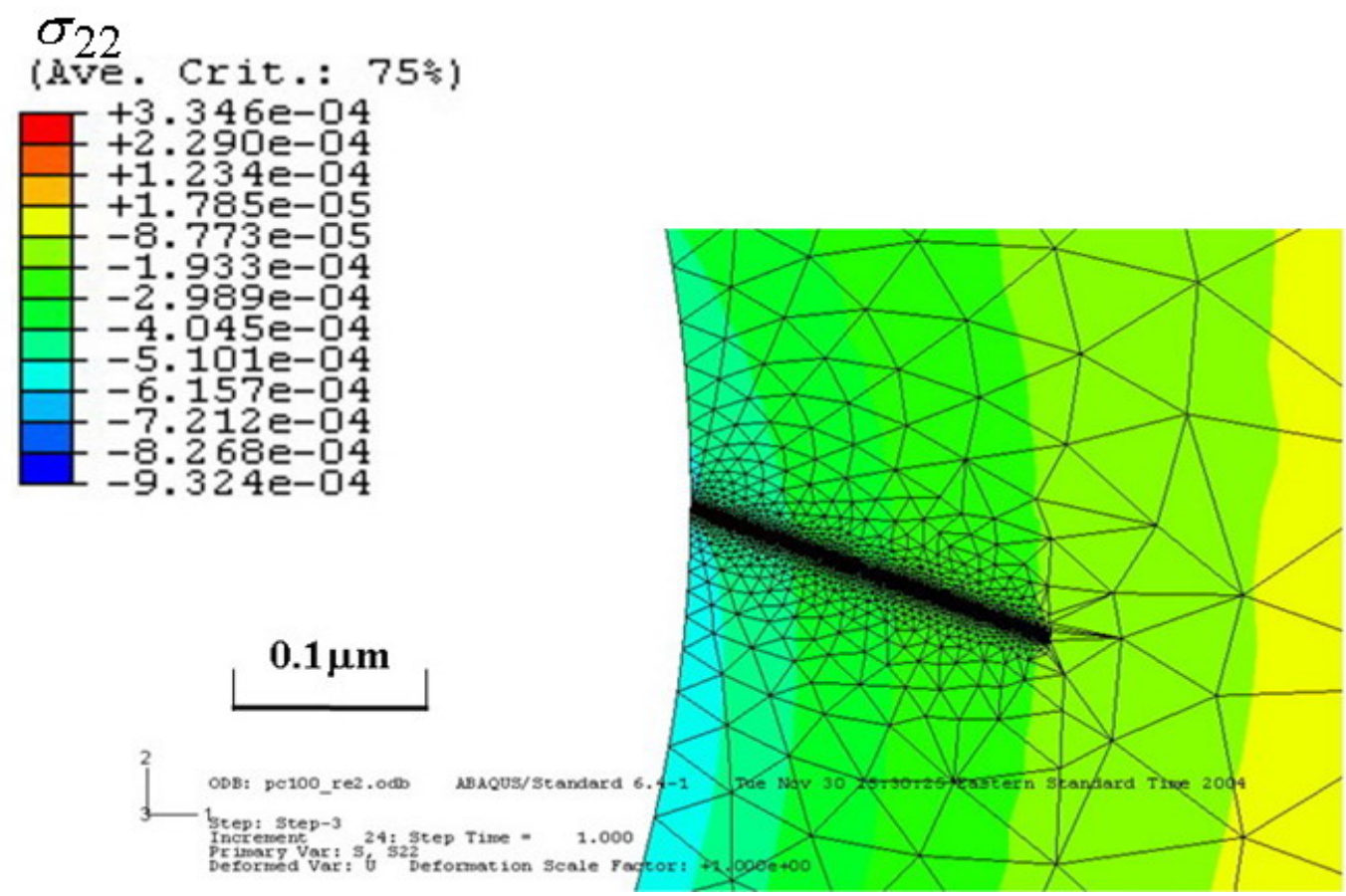

(b)

Figure 6.11 Residual stress distribution in notched polysilicon MEMS specimen after 1000 cycles; (a) $\sigma_{\mathrm{m}}=2.0 \mathrm{GPa}, \Delta \sigma=2.0 \mathrm{GPa}$, (b) $\sigma_{\mathrm{m}}=-3.5 \mathrm{GPa}, \Delta \sigma=2.0 \mathrm{GPa}$. 


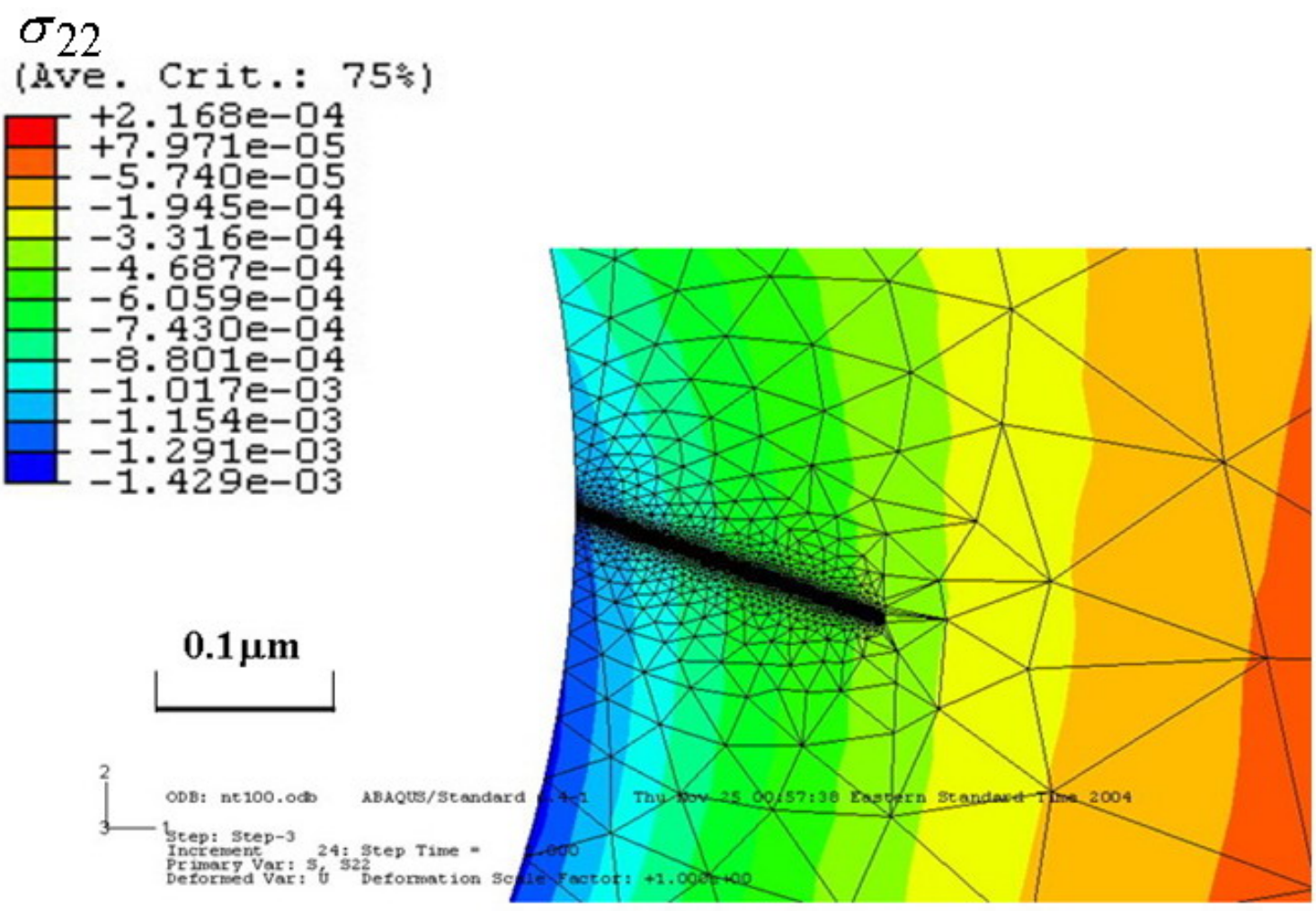

Figure 6.12 Residual stress distribution in notched polysilicon MEMS specimen after 1000 cycles; $\sigma_{\mathrm{m}}=2.0 \mathrm{GPa}, \Delta \sigma=2.0 \mathrm{GPa}\left(\psi=35^{\circ}\right)$. 


\section{Appendix A}

\section{A.1 Process flow}

Table A.1 lists a process flow designed for fabricating the microstructure. A brief description for each step is also presented in this table. The fabrication parameters are estimated values based on the successful experience of previous processes. During the actual fabrication, new problems will definitely arise due to the complexity of the designed microstructure. Appropriate adjustment of the process flow is needed throughout the whole fabrication procedure, and the actual process parameters will be determined by using testing experiments.

\begin{tabular}{|l|l|l|l|}
\hline Step & Process & Note & Time \\
\hline 1 & RCA clean & $\begin{array}{l}\text { Single side polished bare Si } \\
(100) \text { wafer, diameter } \\
100.0 \mathrm{~mm}, \text { dopant type/P- } \\
\text { type Boron, thickness } 455- \\
575 \mu \mathrm{m}\end{array}$ & 3 Hours \\
\hline 2 & $\begin{array}{l}\text { Thermal oxidation } \\
\text { Tube 322-2 } \\
\text { Target thickness } 2.0 \mu \mathrm{m}\end{array}$ & $\begin{array}{l}\text { Thermal silicon dioxide } \\
\text { deposition at temperature of } \\
1075^{0} \mathrm{C} \text { for } 10 \text { hours. }\end{array}$ & 14 Hours \\
\hline 3 & $\begin{array}{l}\text { Measure oxide thickness on } \\
\text { ellipsometer. }\end{array}$ & & 1 Hour \\
\hline 4 & $\begin{array}{l}\text { RCA clean } \\
\text { (If necessary) }\end{array}$ & 3 Hours \\
\hline
\end{tabular}




\begin{tabular}{|c|c|c|c|}
\hline 5 & $\begin{array}{l}\text { Deposit undoped polysilicon } \\
\text { by LPCVD } \\
\text { Tube } 321-2 \\
\text { Target thickness } 2.5 \mu \mathrm{m}\end{array}$ & $\begin{array}{l}\text { At temperature of } 617^{\circ} \mathrm{C} \text { for } \\
315 \text { minutes with a } \\
\text { deposition rate of } 95 \AA / \mathrm{min} \text {. }\end{array}$ & 11 Hours \\
\hline 6 & $\begin{array}{l}\text { Measure film thickness of } \\
\text { Polysilicon on oxide film, } \\
\text { using ellipsometer. }\end{array}$ & & 1 Hour \\
\hline 7 & $\begin{array}{l}\text { HMDS } \\
\text { Photoresist } \\
\text { Soft bake } \\
\text { Align/Expose/Develop } \\
\text { (mask1) } \\
\text { Hard bake } \\
\end{array}$ & $\begin{array}{l}\text { Spinner speed } 4000 \mathrm{rpm} \text { for } \\
1.3 \mu \mathrm{m} \text { thick photoresist. } \\
\text { Soft bake: } 30 \text { mins } / 95{ }^{\circ} \mathrm{C} \\
\text { Expose time } 2 \mathrm{Seconds} \\
\text { Hard bake: } 30 \text { mins } / 115^{\circ} \mathrm{C}\end{array}$ & 4 Hours \\
\hline 8 & $\begin{array}{l}\text { RIE etch ploysilicon in Lam } \\
\text { Etch stop on oxide }\end{array}$ & $\begin{array}{l}\text { Chlorine as etching gas, etch } \\
\text { rate is about } 3000 \AA / \mathrm{min} \text {. }\end{array}$ & 3 Hours \\
\hline 9 & $\begin{array}{l}\text { Remove photoresist using } \\
\text { Piranha clean process }\end{array}$ & & 1 Hour \\
\hline 10 & $\begin{array}{l}\text { HMDS } \\
\text { Photoresist } \\
\text { Soft bake } \\
\text { Align/Expose/Develop } \\
\text { (mask3) } \\
\text { Hard bake } \\
\end{array}$ & $\begin{array}{l}\text { Spinner speed } 6000-7000 \\
\text { rpm for } 1.0 \mu \mathrm{m} \text { thick } \\
\text { photoresist } \\
\text { Soft bake: } 30 \text { mins } / 95{ }^{\circ} \mathrm{C} \\
\text { Expose time } 1.6 \text { Seconds } \\
\text { Hard bake: } 30 \text { mins } / 115^{\circ} \mathrm{C}\end{array}$ & 6 Hours \\
\hline 11 & $\begin{array}{l}\text { Photoresist etching with RIE } \\
\text { in Tegal }\end{array}$ & $\begin{array}{l}\mathrm{CHF}_{3}, \mathrm{O}_{2} \text { and Helium as } \\
\text { etching gases with a etch } \\
\text { rate of } 4000 \AA / \mathrm{min} \text {. }\end{array}$ & 3 Hours \\
\hline 12 & Sputter doped-Silicon & $\begin{array}{l}\text { Doped-Silicon target with a } \\
\text { purity of } 99.999 \% \text {. Deposit } \\
3.0 \mu \mathrm{m} \text { thick silicon film } \\
\text { with rate of } 100 \AA \mathrm{min} \text {. }\end{array}$ & 4 Hours \\
\hline 13 & $\begin{array}{l}\text { HMDS } \\
\text { Photoresist } \\
\text { Soft bake } \\
\text { Align/Expose/Develop } \\
\text { (mask2) } \\
\text { Hard bake }\end{array}$ & $\begin{array}{l}\text { Spinner speed 4000rpm for } \\
1.3 \mu \mathrm{m} \text { thick photoresist. } \\
\text { Soft bake: } 30 \text { mins } / 95{ }^{\circ} \mathrm{C} \\
\text { Expose time } 2 \text { Seconds } \\
\text { Hard bake: } 30 \text { mins } / 115^{0} \mathrm{C}\end{array}$ & 6 Hours \\
\hline 14 & Etch sputtered Silicon in Lam & $\begin{array}{l}\text { Chlorine as etching gas, etch } \\
\text { rate is about } 3000 \AA / \mathrm{min} \text {. }\end{array}$ & 3 Hours \\
\hline
\end{tabular}




\begin{tabular}{|c|c|c|c|}
\hline 15 & $\begin{array}{l}\text { Photoresist etching with RIE } \\
\text { in Tegal }\end{array}$ & $\begin{array}{l}\mathrm{CHF}_{3}, \mathrm{O}_{2} \text { and Helium as } \\
\text { etching gases with a etch } \\
\text { rate of } 4000 \AA / \mathrm{min} \text {. }\end{array}$ & 3 Hour \\
\hline 16 & $\begin{array}{l}\text { HMDS } \\
\text { Photoresist } \\
\text { Soft bake } \\
\text { Align/Expose/Develop } \\
\text { (mask3) } \\
\text { Hard bake }\end{array}$ & $\begin{array}{l}\text { Spinner speed } 6000-7000 \\
\text { rpm for } 1.0 \mu \mathrm{m} \text { thick } \\
\text { photoresist } \\
\text { Soft bake: } 30 \text { mins } / 95{ }^{0} \mathrm{C} \\
\text { Expose time } 1.6 \mathrm{Seconds} \\
\text { Hard bake: } 30 \text { mins } / 115^{0} \mathrm{C}\end{array}$ & 6 Hours \\
\hline 17 & $\begin{array}{l}\text { Photoresist etching with RIE } \\
\text { in Tegal }\end{array}$ & $\begin{array}{l}\mathrm{CHF}_{3}, \mathrm{O}_{2} \text { and Helium as } \\
\text { etching gases with a etch } \\
\text { rate of } 4000 \AA / \mathrm{min} \text {. }\end{array}$ & 3 Hours \\
\hline 18 & Sputter doped-Silicon & $\begin{array}{l}\text { Doped-Silicon target with a } \\
\text { purity of } 99.999 \% \text {. Deposit } \\
3.0 \mu \mathrm{m} \text { thick silicon film } \\
\text { with rate of } 100 \AA \mathrm{min} \text {. }\end{array}$ & 4 Hours \\
\hline 19 & $\begin{array}{l}\text { HMDS } \\
\text { Photoresist application } \\
\text { Soft bake } \\
\text { Align/Expose/Develop } \\
\text { (mask1) } \\
\text { Hard bake }\end{array}$ & $\begin{array}{l}\text { Spinner speed 4000rpm for } \\
1.3 \mu \mathrm{m} \text { thick photoresist. } \\
\text { Soft bake: } 30 \text { mins } / 95{ }^{\circ} \mathrm{C} \\
\text { Expose time } 2 \mathrm{Seconds} \\
\text { Hard bake: } 30 \mathrm{mins} / 115^{\circ} \mathrm{C}\end{array}$ & 6 Hours \\
\hline 20 & Etch sputtered Silicon in Lam & $\begin{array}{l}\text { Chlorine as etching gas, etch } \\
\text { rate is about } 3000 \AA / \mathrm{min} \text {. }\end{array}$ & 3 Hours \\
\hline 21 & $\begin{array}{l}\text { Photoresist etching with RIE } \\
\text { in Tegal }\end{array}$ & $\begin{array}{l}\mathrm{CHF}_{3}, \mathrm{O}_{2} \text { and Helium as } \\
\text { etching gases with a etch } \\
\text { rate of } 4000 \AA / \mathrm{min} \text {. }\end{array}$ & 3 Hour \\
\hline 22 & $\begin{array}{l}\text { HMDS } \\
\text { Thicker Photoresist application } \\
\text { (AZ 9260) } \\
\text { Soft bake } \\
\text { Align/Expose/Develop } \\
\text { (mask4) } \\
\text { Hard bake }\end{array}$ & $\begin{array}{l}\text { Spinner speed } 2500 \mathrm{rpm} \text { for } \\
8.0 \mu \mathrm{m} \text { thick } \mathrm{AZ} \text { photoresist. } \\
\text { Soft bake: } 60 \mathrm{mins} / 95^{\circ} \mathrm{C} \\
\text { Expose time } 12 \mathrm{Seconds} \\
\text { Hard bake: } 60 \text { mins } / 115^{\circ} \mathrm{C}\end{array}$ & 6 Hours \\
\hline 23 & $\begin{array}{l}\text { Etch multi-layered silicon } \\
\text { (sputtered Si/sputtered } \\
\text { Si/Polysilicon) in Lam. }\end{array}$ & $\begin{array}{l}\text { Chlorine as etching gas, etch } \\
\text { rate is about } 3000 \AA / \mathrm{min} \text {. }\end{array}$ & 6 Hours \\
\hline 24 & Dicing & $\begin{array}{l}\text { Dicing wafer by using } \\
\text { diamond saw. }\end{array}$ & 3 Hours \\
\hline 25 & Release structures with BOE. & $\begin{array}{l}\text { Buffered } \\
\text { In MEMS Lab }\end{array}$ & 8 Hours \\
\hline
\end{tabular}

Table A.1 Fabrication procedures. 


\section{A.2 Silicon etching recipe with $\mathrm{SF}_{6} / \mathrm{O}_{2}$ based plasma}

\section{A.2.1 Black silicon}

It is necessary to understand the mechanism for forming the black silicon so that appropriate recipes can be designed. Jansen et al. ${ }^{[21]-[23]}$ studied the origin of the black silicon and described the reasons for its formation. The key factor is the oxygen content in the plasmas etchant and the oxygen concentration in the target film.

When using fluorine-based plasmas to etch silicon, there is a balance between the fluorine radicals used to etch silicon and the oxygen radicals used to form passivation layer in the silicon film. Because of the directional bombarding nature of the plasma etching, passivation layers formed on the sidewall of the trenches will prevent the silicon from being etched laterally. If the content of oxygen is high, the balance will be broken and passivation layers may be also formed at the bottom of the trenches. This will stop the silicon etching in the vertical direction. If the silicon film includes impurities, such as oxygen or native oxide, these impurities will react with etchant and form localized passivation layers, which play as micro-masks distributed randomly in the film. With the help of these localized micro-masks, directional plasmas etching will produce silicon spikes or silicon grass as shown in Figure A.1. 


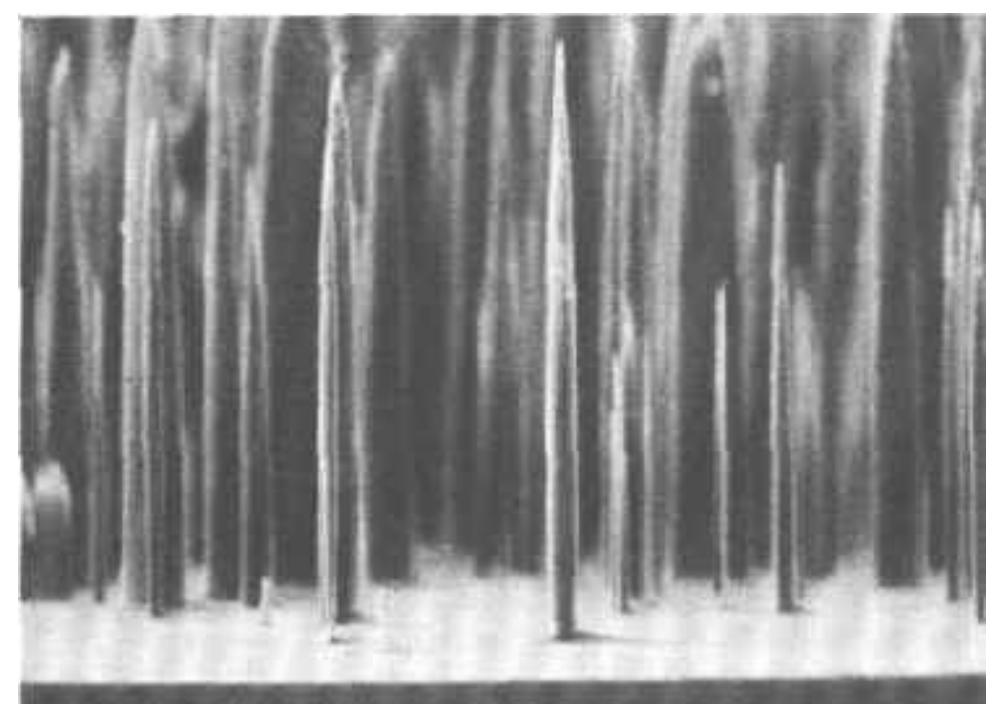

Figure A.1 Black silicon spikes ${ }^{[21]}$.

The black silicon can be etched away using $\mathrm{SF}_{6} / \mathrm{O}_{2}$-based plasma. This can be realized by either adjusting the flow rates of $\mathrm{SF}_{6}$ and $\mathrm{O}_{2}$ or by adding new species into the recipe. Proper combination of these species will make the etching effect dominant over the passivation effect and force the micro-masks to be volatilized.

Jansen et al. took advantage of this idea and added $\mathrm{CHF}_{3}$ into the $\mathrm{SF}_{6} / \mathrm{O}_{2}$ recipe. This is the so-called black silicon method (BSM). This method employs the fact that the silicon will turn black when it is anisotropically etched with $\mathrm{SF}_{6} / \mathrm{O}_{2}$ plasma. As shown in Figure A.2, $\mathrm{F}^{*}$ radicals react with silicon and form volatile $\mathrm{SiF}_{4}$. Both the oxygen radicals and the fluorine radicals are used to passivate the exposed silicon surface with $\mathrm{SiO}_{\mathrm{x}} \mathrm{F}_{\mathrm{y}}$. Once the sidewalls of the trenches are fully covered with $\mathrm{SiO}_{\mathrm{x}} \mathrm{F}_{\mathrm{y}}$ and the silicon turns black, an anisotropic etching state is reached. Namely, the appearance of the black silicon represents the critical point after which the etching process is completely anisotropic. Thereafter, $\mathrm{CHF}_{3}$ is added into the $\mathrm{SF}_{6} / \mathrm{O}_{2}$ plasma. It provides $\mathrm{CF}_{\mathrm{x}}{ }^{+}$ions for reacting with $\mathrm{SiO}_{\mathrm{x}} \mathrm{F}_{\mathrm{y}}$ and forming volatile $\mathrm{CO}_{\mathrm{x}} \mathrm{F}_{\mathrm{y}}$. 


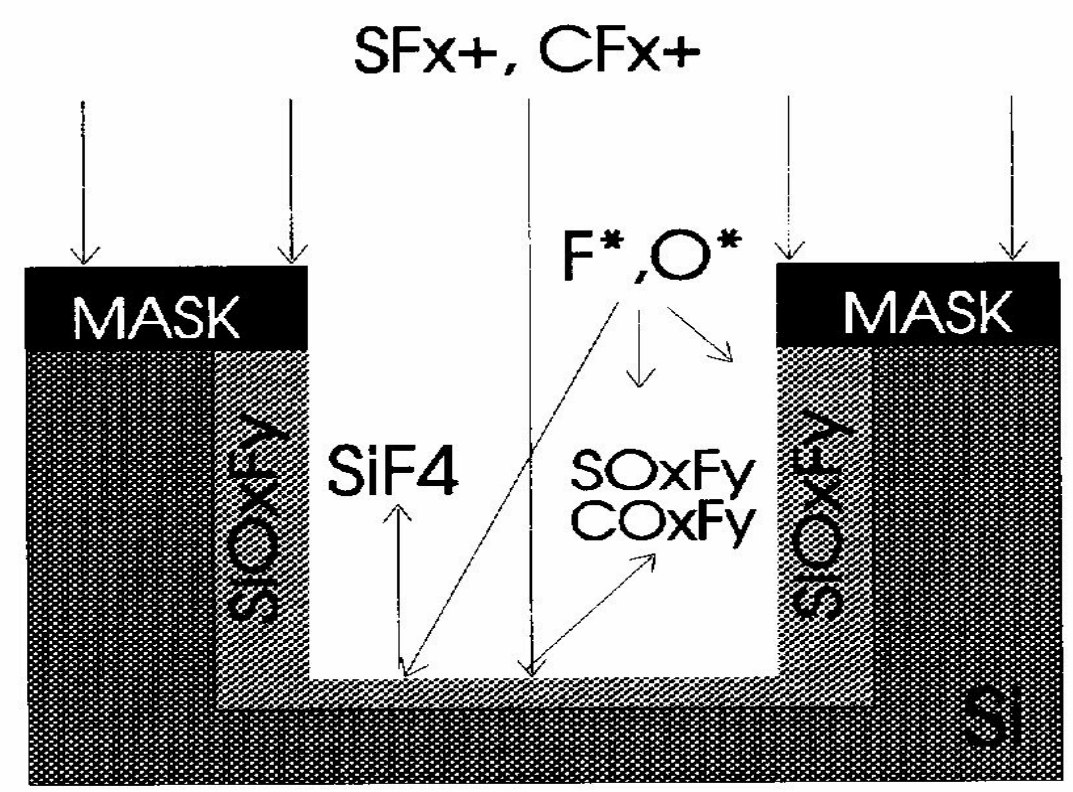

Figure A.2 Etching mechanism of the black silicon method ${ }^{[21]}$.

For different etching processes, the flow rates of $\mathrm{SF}_{6}, \mathrm{O}_{2}$ and $\mathrm{CHF}_{3}$ need to be adjusted to get best etching results. The objective of the adjustment is to find the critical point, and then remove the passivation materials and micro-masks by adding $\mathrm{CHF}_{3}$ to certain level to enhance the etching plasma. With this method, the etch rate can reach $5 \mu \mathrm{m} / \mathrm{min}$. Since different reactors have different designs and working chambers, the recipe does not include fixed gas content.

\section{A.2.2 Trial Recipe-1}

The idea of the black silicon method can be employed to etch black silicon in the etched sputtered silicon film. In LAM490, the existing gas configuration does not include 
$\mathrm{CHF}_{3}$. Therefore, the flow rates of $\mathrm{SF}_{6}$ and $\mathrm{O}_{2}$ will be adjusted to obtain a proper flow ratio for etching the black silicon.

Since the combination of $\mathrm{SF}_{6} / \mathrm{O}_{2}$ has never been practiced in LAM490 before, the typical recipes provided by Jansen et al. ${ }^{[22]}$ will be used as reference. Two of them are: power $=75 \mathrm{Watts}$, pressure $=100 \mathrm{mTorr}, \mathrm{SF}_{6} / \mathrm{O}_{2} / \mathrm{CHF}_{3}=28 / 15 / 21 \mathrm{sccm}$ and power $=$ 75 Watts, pressure $=75 \mathrm{mTorr}, \mathrm{SF}_{6} / \mathrm{O}_{2} / \mathrm{CHF}_{3}=27 / 11 / 21 \mathrm{sccm}$. The ratio of the flow rate for $\mathrm{SF}_{6}$ and $\mathrm{O}_{2}$ is close to 2 . This ratio will be used for the first trial recipe.

\begin{tabular}{|l|c|c|c|c|}
\hline & Step1 & Step2 & Step3 & Step4 \\
\hline Pressure (mTorr) & 500 & 500 & 400 & 400 \\
\hline RF Top Power (Watt) & 0 & 300 & 0 & 200 \\
\hline Gap $(\mathrm{cm})$ & 0.8 & 0.8 & 0.6 & 0.6 \\
\hline $\mathrm{Cl}_{2}(\mathrm{sccm})$ & 0 & 0 & 80 & 80 \\
\hline $\mathrm{O}_{2}(\mathrm{sccm})$ & 0 & 0 & 0 & 0 \\
\hline Helium $(\mathrm{sccm})$ & 0 & 0 & 120 & 120 \\
\hline $\mathrm{C}_{2} \mathrm{~F}_{6}(\mathrm{sccm})$ & 100 & 100 & 0 & 0 \\
\hline $\mathrm{SF}_{6}(\mathrm{sccm})$ & 0 & 0 & 0 & 0 \\
\hline Function & Stability & Time & Stability & Time \\
\hline Time $($ second) & 20 & 20 & 20 & Varies with process \\
\hline
\end{tabular}

Table A.2 Details of chlorine-based silicon etching recipe.

The trial recipe was developed by referring the basic steps of the chlorine-based recipe in LAM490 as listed in Table A.2. The first and second steps were designed for removing native oxide using $\mathrm{C}_{2} \mathrm{~F}_{6}$ plasmas. In the trial recipe-1, they were retained. Step 3 was a pre-etching process to stabilize the gas flow. In this step, helium was pumped into the chamber as a carrier gas to help the etching gases to flow in the chamber. At this point, the power was turned off so that no etching process was conducted. The trial recipe-1 started taking effect from this step. Instead of using $\mathrm{Cl}_{2}, \mathrm{SF}_{6}$ and $\mathrm{O}_{2}$ were 
pumped into the chamber. The flow rates for $\mathrm{SF}_{6}$ and $\mathrm{O}_{2}$ were set to be $40 \mathrm{sccm}$ and $20 \mathrm{sccm}$, respectively. Helium was still used for carrier gas. In step 4, the power was turned on and etching process started. Table A.3 shows the details of the trial recipe-1. The trial recipe- 1 was run for several times upon one sputtered silicon film to study the etching properties. Since the first two steps were used for removing native oxide and $\mathrm{C}_{2} \mathrm{~F}_{6}$ etched silicon isotropically, they were disengaged for the second and latter runs.

\begin{tabular}{|l|c|c|c|c|}
\hline & Step1 & Step2 & Step3 & Step4 \\
\hline Pressure (mTorr) & 500 & 500 & 400 & 400 \\
\hline $\mathrm{RF}$ Top Power (Watt) & 0 & 300 & 0 & 200 \\
\hline $\mathrm{Gap}(\mathrm{cm})$ & 0.8 & 0.8 & 0.6 & 0.6 \\
\hline $\mathrm{Cl}_{2}(\mathrm{sccm})$ & 0 & 0 & 0 & 0 \\
\hline $\mathrm{O}_{2}(\mathrm{sccm})$ & 0 & 0 & 20 & 20 \\
\hline Helium $(\mathrm{sccm})$ & 0 & 0 & 120 & 120 \\
\hline $\mathrm{C}_{2} \mathrm{~F}_{6}(\mathrm{sccm})$ & 100 & 100 & 0 & 0 \\
\hline $\mathrm{SF}_{6}(\mathrm{sccm})$ & 0 & 0 & 40 & 40 \\
\hline Function & Stability & Time & Stability & Time \\
\hline Time (second) & 20 & 20 & 20 & Varies with process \\
\hline
\end{tabular}

Table A.3 Trial recipe-1

First, the polysilicon film was etched to study the etching uniformity and collect data for estimating the etch rate. The testing wafers were prepared with $2 \mu \mathrm{m}$ thick thermally grown silicon dioxide film and $3 \mu \mathrm{m}$ thick LPCVD polysilicon film. For the first test, no etching mask was used and the completely exposed polysilicon film was etched for one minute. After that, most of the polysilicon film was gone and only a tiny part around the center was left. This indicates that the etch rate was faster than $3 \mu \mathrm{m} / \mathrm{min}$ and the etching uniformity was not very good. Another test was run for 30 seconds, and then fringes appeared on the wafer as sketched in Figure A.3. 
The wafer surface included three major fringes as labeled in Figure A.3. Area 1 was close to the wafer edge and had a width $\mathrm{t} 1$ of about $5 \mathrm{~mm}$. The polysilicon thickness in this area was about zero. Area 2 was wider than area 1 and had a width of $1.3 \mathrm{~cm}$. The polysilicon thickness in this area varied from $800 \AA$ to $10000 \AA$, from outside edge to inside edge. Area 3 had a polysilicon thickness of $15000 \AA$ around the wafer center.

These data clearly sketch a non-uniform etching profile across the wafer radius. The etch rate varies very much from the wafer edge to the wafer center. This might have been caused by lower gas flow rate in the etching chamber. As the etch rate is very high, the low flow rate cannot continuously provide enough plasma and will delay the etching process in the region with lower plasma concentration. In the chlorine-based recipe, the gas flow rate is $80 \mathrm{sccm}$, which is larger than the summation of the flow rates of $\mathrm{SF}_{6}$ and $\mathrm{O}_{2}$.

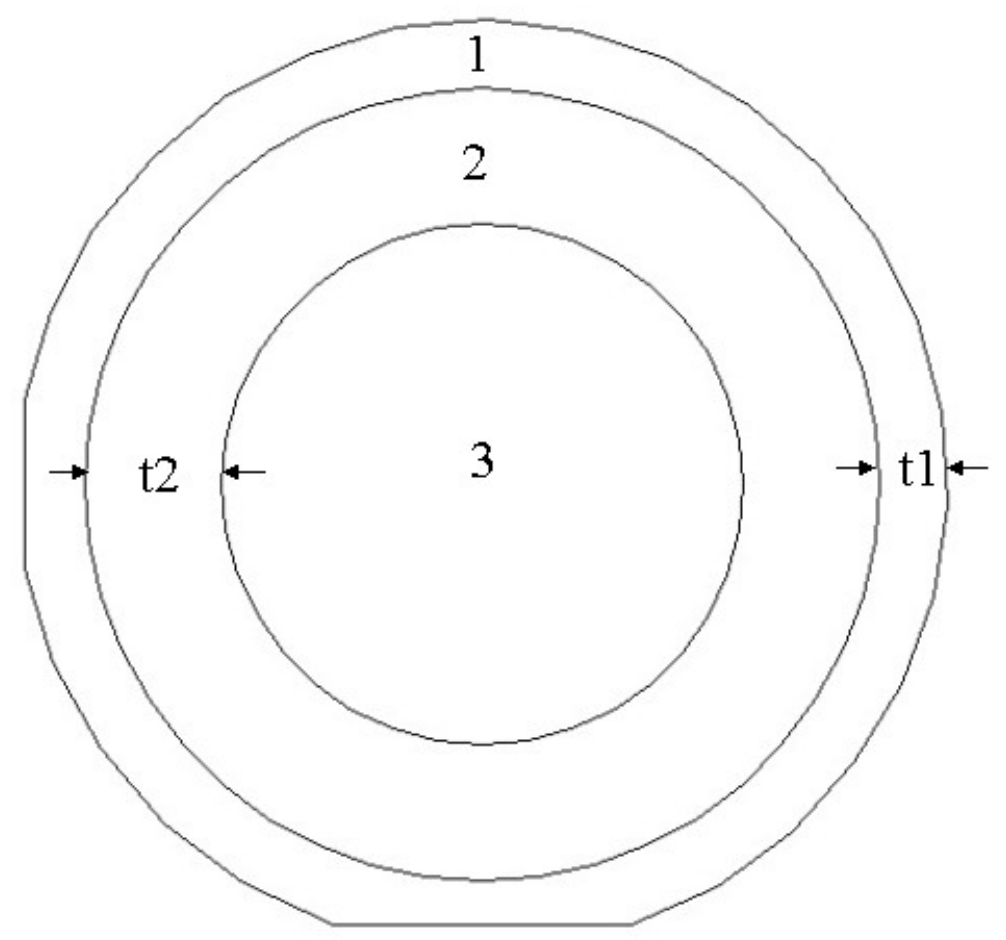

Figure A.3 Sketch of the etching results for polysilicon film using trial recipe-1. 


\section{A.2.3 Trial Recipe-2}

In order to overcome the drawback included in the trial recipe-1, the flow rates were increased to $80 \mathrm{sccm}$ and $40 \mathrm{sccm}$ for $\mathrm{SF}_{6}$ and $\mathrm{O}_{2}$. As higher power may help to form uniform and stable plasma, 300Watts was used in step 4. In step3, longer stabilizing time was set to further improve the uniformity of the gas distribution in the chamber. The details of the trial recipe- 2 are shown in Table A.4.

\begin{tabular}{|l|c|c|c|c|}
\hline & Step1 & Step2 & Step3 & Step4 \\
\hline Pressure (mTorr) & 500 & 500 & 400 & 400 \\
\hline RF Top Power (Watt) & 0 & 300 & 0 & 300 \\
\hline Gap $(\mathrm{cm})$ & 0.8 & 0.8 & 0.6 & 0.6 \\
\hline $\mathrm{Cl}_{2}(\mathrm{sccm})$ & 0 & 0 & 0 & 0 \\
\hline $\mathrm{O}_{2}(\mathrm{sccm})$ & 0 & 0 & 40 & 40 \\
\hline $\mathrm{Helium}^{(\mathrm{sccm})}$ & 0 & 0 & 120 & 120 \\
\hline $\mathrm{C}_{2} \mathrm{~F}_{6}(\mathrm{sccm})$ & 100 & 100 & 0 & 0 \\
\hline $\mathrm{SF}_{6}(\mathrm{sccm})$ & 0 & 0 & 80 & 80 \\
\hline Function & Stability & Time & Stability & Time \\
\hline Time (second) & 20 & 20 & 30 & Vary with process \\
\hline
\end{tabular}

Table A.4 Trial recipe-2 


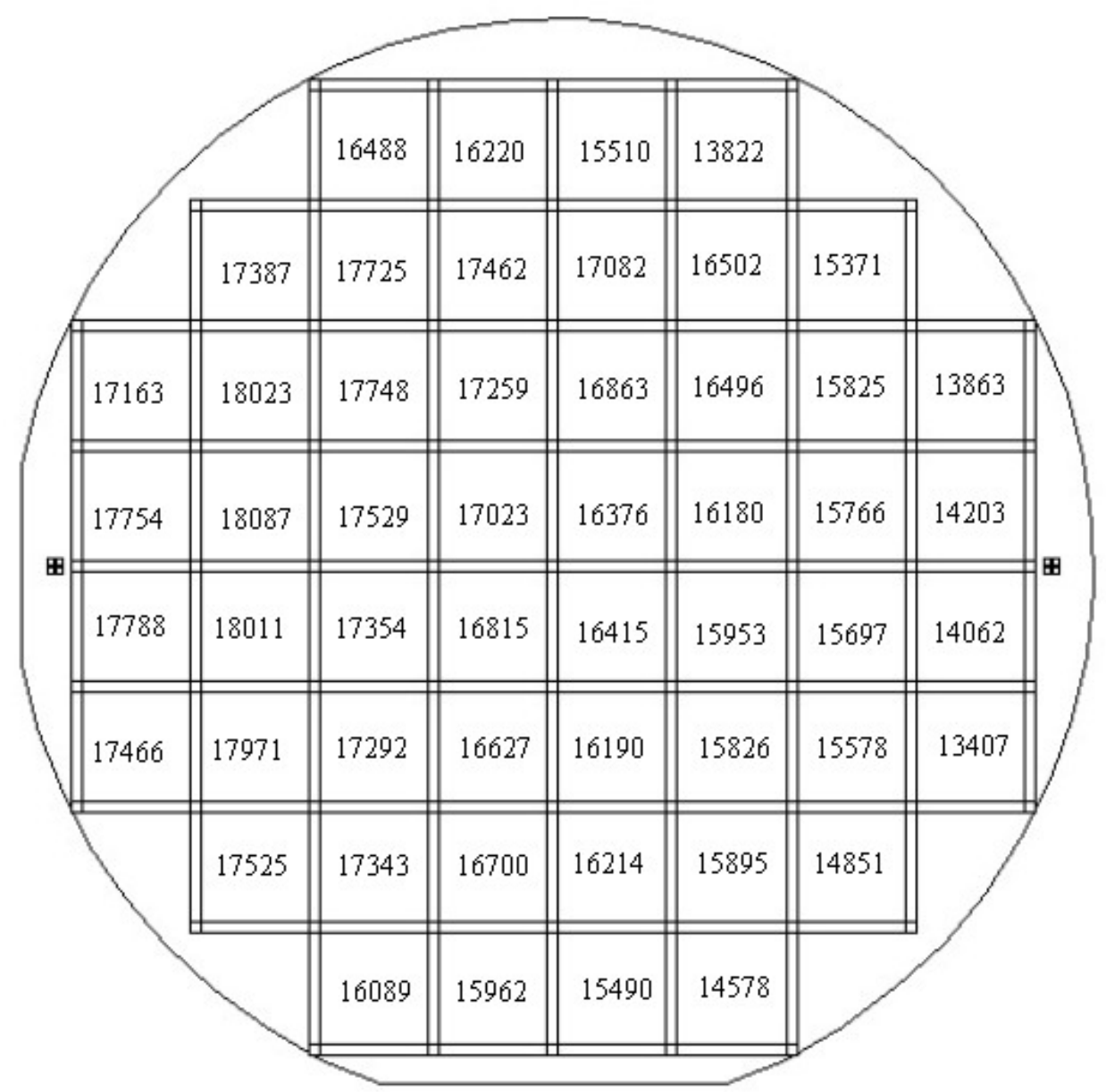

Figure A.4 Polysilicon film thicknesses after 20 seconds etching using trial recipe-2.

After being etched for 20 seconds, the wafer surface was relatively smooth even though dense fringes still could be seen around the wafer edge. The etching uniformity was significantly improved with the modified recipe. Polysilicon film thickness was measured across the whole wafer, not just 5 points for general measurement.

Figure A.4 shows the average thickness in each small die. If only the central $4 x 4$ dice in Figure A.4 are considered, the average thickness is $16836 \AA$, which results in an etch rate of $39492 \AA / \mathrm{min}$ with a uniformity of $6 \%$. This is an acceptable etching profile. 
The trial recipe- 2 produces much better etching results compared to the trial recipe- 1 . However, there is still an etching profile gradient from the center to the edge.

The etching power, the chamber pressure and the gap between wafer stage and cathode were adjusted for solving the uniformity problem, but no significant improvement was obtained. Thus, the trial recipe-2 was accepted for further tests.

Next, photomask 'odd layer' was used to pattern the $1.2 \mu \mathrm{m}$ thick photoresist mask. The polysilicon film was etched for 20 seconds using trial recipe-2. No obvious black silicon was found in the etched area. However, some etched areas turned rough and grey. Scattered black spots were found in the trenches as shown in Figure A.5.

The film was etched for another 20 seconds, and then even more black spots appeared in the etched area as shown in Figure A.6. Photoresist was removed after 40 seconds etching and the feature step heights were measured. The testing data are listed in Table A.5.

\begin{tabular}{|l|l|l|l|l|l|}
\hline & M1 $(\AA)$ & M2 $(\AA)$ & M3 $(\AA)$ & M4 $(\AA)$ & M5 $(\AA)$ \\
\hline After 20 seconds etching & 19021 & 21237 & 20854 & 21086 & 22339 \\
\hline After 40 seconds etching & 23452 & 24875 & 24608 & 23117 & 25332 \\
\hline $\begin{array}{l}\text { After removing photoresist } \\
\text { mask }\end{array}$ & 15663 & 17029 & 16114 & 15247 & 16873 \\
\hline
\end{tabular}

Table A.5 Etching results of the polysilicon film (trial recipe-2 and photomask 'odd layer'). 


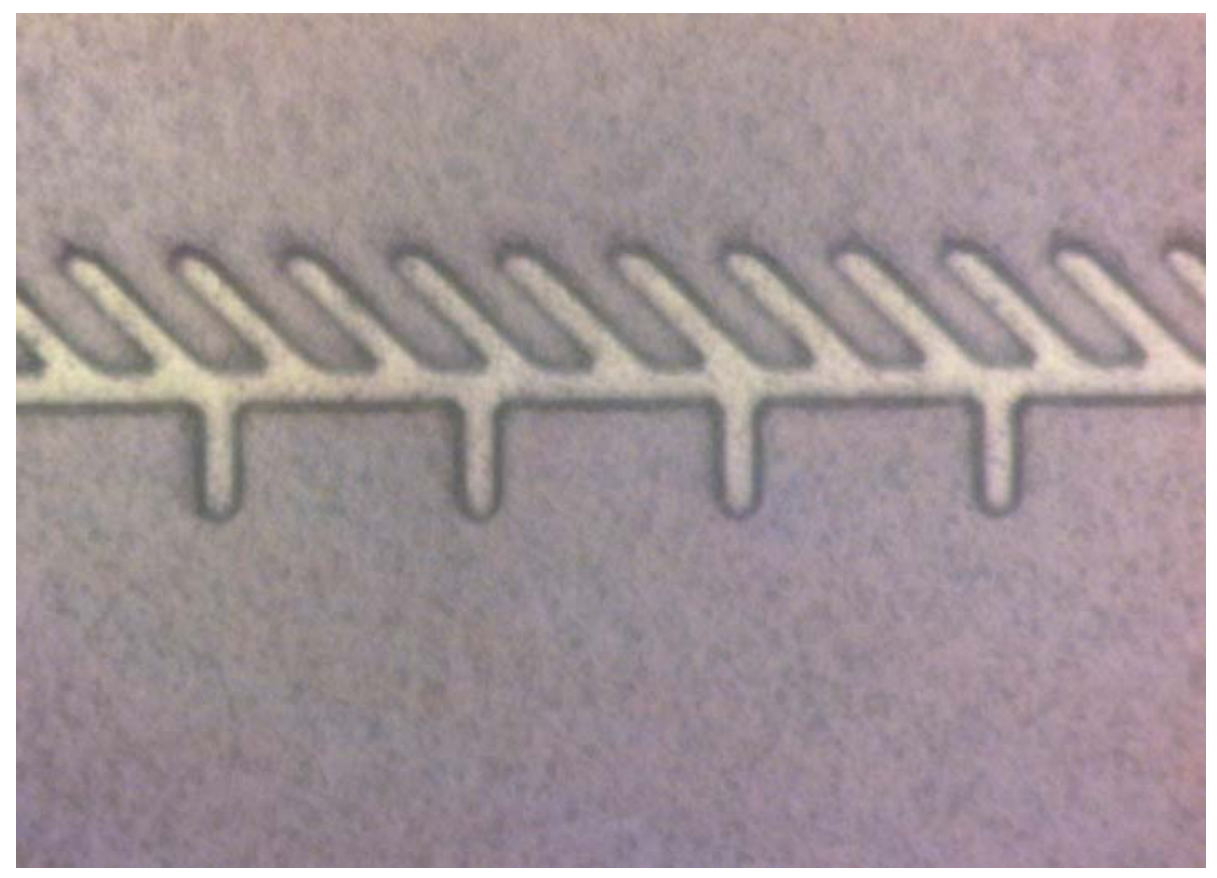

Figure A.5 Black spots in the trenches after 20secs etching for the polysilicon film (trial recipe-2, photomask 'odd layer').

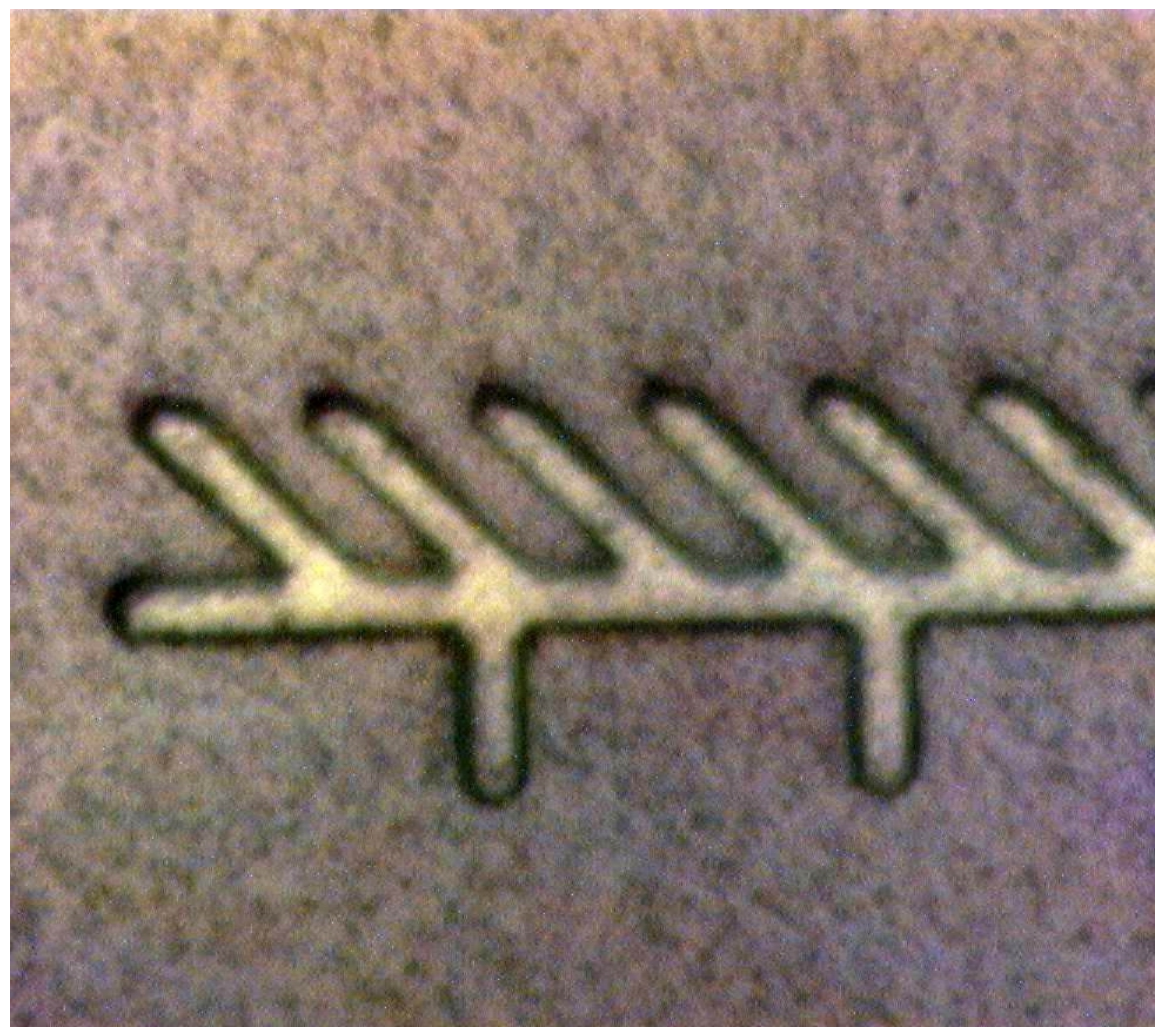

Figure A.6 Black spots in the trenches after 40sec etching for the polysilicon film (trial recipe-2, photomask 'odd layer'). 
Assuming the etch rate of photoresist is consistent within all of the etching steps, the photoresist etch rate can be obtained by subtracting the difference between the third row and the fourth row of Table A.5 from the total thickness of the photoresist film, which is $1.2 \mu \mathrm{m}$. Thereafter, the silicon etch rate in each step can be determined with respect to the photoresist etch rate. The calculated data are shown in Table A.6.

\begin{tabular}{|l|l|l|l|l|l|}
\hline & $\begin{array}{l}\text { M1 } \\
(\AA / \mathrm{min})\end{array}$ & $\begin{array}{l}\text { M2 } \\
(\AA / \mathrm{min})\end{array}$ & $\begin{array}{l}\text { M3 } \\
(\AA / \mathrm{min})\end{array}$ & $\begin{array}{l}\text { M4 } \\
(\AA / \mathrm{min})\end{array}$ & $\begin{array}{l}\text { M5 } \\
(\AA / \mathrm{min})\end{array}$ \\
\hline Photoresist Etch rate & 6316 & 6231 & 5259 & 6195 & 5312 \\
\hline $\begin{array}{l}\text { Silicon Etch rate in the first 20 } \\
\text { seconds }\end{array}$ & 27379 & 33942 & 31821 & 33453 & 36329 \\
\hline $\begin{array}{l}\text { Silicon Etch rate in the second 20 } \\
\text { seconds }\end{array}$ & 19610 & 17145 & 16521 & 12288 & 14290 \\
\hline
\end{tabular}

Table A.6 Etch rates of the polysilicon film using trial recipe-2.

The silicon etch rate drops in the second run. This can be explained because the passivation effect tends to dominate the process, as evidenced by the appearance of more black spots in the trenches.

The same testing processes were run on the sputtered silicon films to verify the etching consistency. Two microns thick sputtered silicon film was prepared and the film was covered by the photomask 'odd layer'. It was etched for 20 seconds using trial recipe-2. Almost the same etching result as shown in Figure A.6 was obtained. No continuous black silicon appeared, but dense black spots scattered in the etched area.

Step height measurements in Table A.7 imply that the etch rate of the sputtered silicon film is slower than that of the polysilicon film, while the etch rate of the photoresist film is consistent in terms of the comparison of Table A.6 and Table A.8. 


\begin{tabular}{|l|l|l|l|l|l|}
\hline & M1 $(\AA)$ & M2 $(\AA)$ & M3 $(\AA)$ & M4 $(\AA)$ & M5 $(\AA)$ \\
\hline After 20 seconds etching & 17748 & 18298 & 17418 & 19109 & 17767 \\
\hline After removing photoresist mask & 7521 & 8843 & 7759 & 9066 & 8024 \\
\hline
\end{tabular}

Table A.7 Etching results of the sputtered silicon film (trial recipe-2, photomask 'odd layer').

\begin{tabular}{|l|l|l|l|l|l|}
\hline & $\begin{array}{l}\text { M1 } \\
(\AA / \mathrm{min})\end{array}$ & $\begin{array}{l}\text { M2 } \\
(\AA / \mathrm{min})\end{array}$ & $\begin{array}{l}\text { M3 } \\
(\AA / \mathrm{min})\end{array}$ & $\begin{array}{l}\text { M4 } \\
(\AA / \mathrm{min})\end{array}$ & $\begin{array}{l}\text { M5 } \\
(\AA / \mathrm{min})\end{array}$ \\
\hline Photoresist Etch rate & 5319 & 7635 & 7022 & 5869 & 6771 \\
\hline $\begin{array}{l}\text { Silicon Etch rate in the first } 20 \\
\text { seconds }\end{array}$ & 22563 & 26529 & 23277 & 27198 & 24072 \\
\hline
\end{tabular}

Table A.8 Etch rates of the sputtered silicon film (trial recipe-2, photomask 'odd layer').

The lower etch rate of the sputtered silicon film demonstrates that the passivation effect is prominent during the etching process using trial recipe-2. That makes sense because the sputtered silicon film is incorporated with oxygen impurity. The pronounced oxygen concentration makes the reaction balance more close to the passivation side.

\section{A.2.4 Trial Recipe-3}

Generally, increasing the content of oxygen in the plasma will lead the etching process to the direction of forming black silicon. On the other hand, the recipe with lower proportion of oxygen will etch silicon rapidly with no black silicon left behind. It is necessary to lower the flow rate of oxygen in the trial recipe- 2 in order to weaken the passivation effect. 
As shown in Table A.9, the new recipe, trial recipe-3, has a ratio of 4 for the flow rates of $\mathrm{SF}_{6}$ and $\mathrm{O}_{2}$. The total gas flow rate is kept the same as the trial recipe- 2 .

\begin{tabular}{|l|c|c|c|c|}
\hline & Step1 & Step2 & Step3 & Step4 \\
\hline Pressure (mTorr) & 500 & 500 & 400 & 400 \\
\hline RF Top Power (Watt) & 0 & 300 & 0 & 300 \\
\hline Gap $(\mathrm{cm})$ & 0.8 & 0.8 & 0.6 & 0.6 \\
\hline $\mathrm{Cl}_{2}(\mathrm{sccm})$ & 0 & 0 & 0 & 0 \\
\hline $\mathrm{O}_{2}(\mathrm{sccm})$ & 0 & 0 & 24 & 24 \\
\hline Helium $(\mathrm{sccm})$ & 0 & 0 & 120 & 120 \\
\hline $\mathrm{C}_{2} \mathrm{~F}_{6}(\mathrm{sccm})$ & 100 & 100 & 0 & 0 \\
\hline $\mathrm{SF}_{6}(\mathrm{sccm})$ & 0 & 0 & 96 & 96 \\
\hline Function & Stability & Time & Stability & Time \\
\hline Time (second) & 20 & 20 & 30 & Varies with process \\
\hline
\end{tabular}

Table A.9 Trial recipe-3

The same tests as described in section A.2.2 were conducted with the trial recipe3. The etching results are presented in Table A.10 A.13. Figure A.7 and Figure A.8 are the top views of the trench areas for the polysilicon film and the sputtered silicon film after being etched for 40 seconds and 20 seconds, respectively. No black silicon appears in the etched area for both polysilicon and sputtered silicon. The trial recipe-3 provides consistent etch rate through multi-step etching processes. The etch rate for polysilicon varies from $36000 \AA / \mathrm{min}$ to $42000 \AA / \mathrm{min}$ and the mean is $37469 \AA / \mathrm{min}$ with a uniformity of $8.06 \%$. It shows that the trial recipe- 3 etches the edge area faster than the central area. It is consistent with the trial recipe- 1 and the trial recipe-2. For the sputtered silicon film, the average etch rate is $34536 \AA / \mathrm{min}$ with a uniformity of $8.46 \%$, which is relatively lower than that of the polysilicon film. 


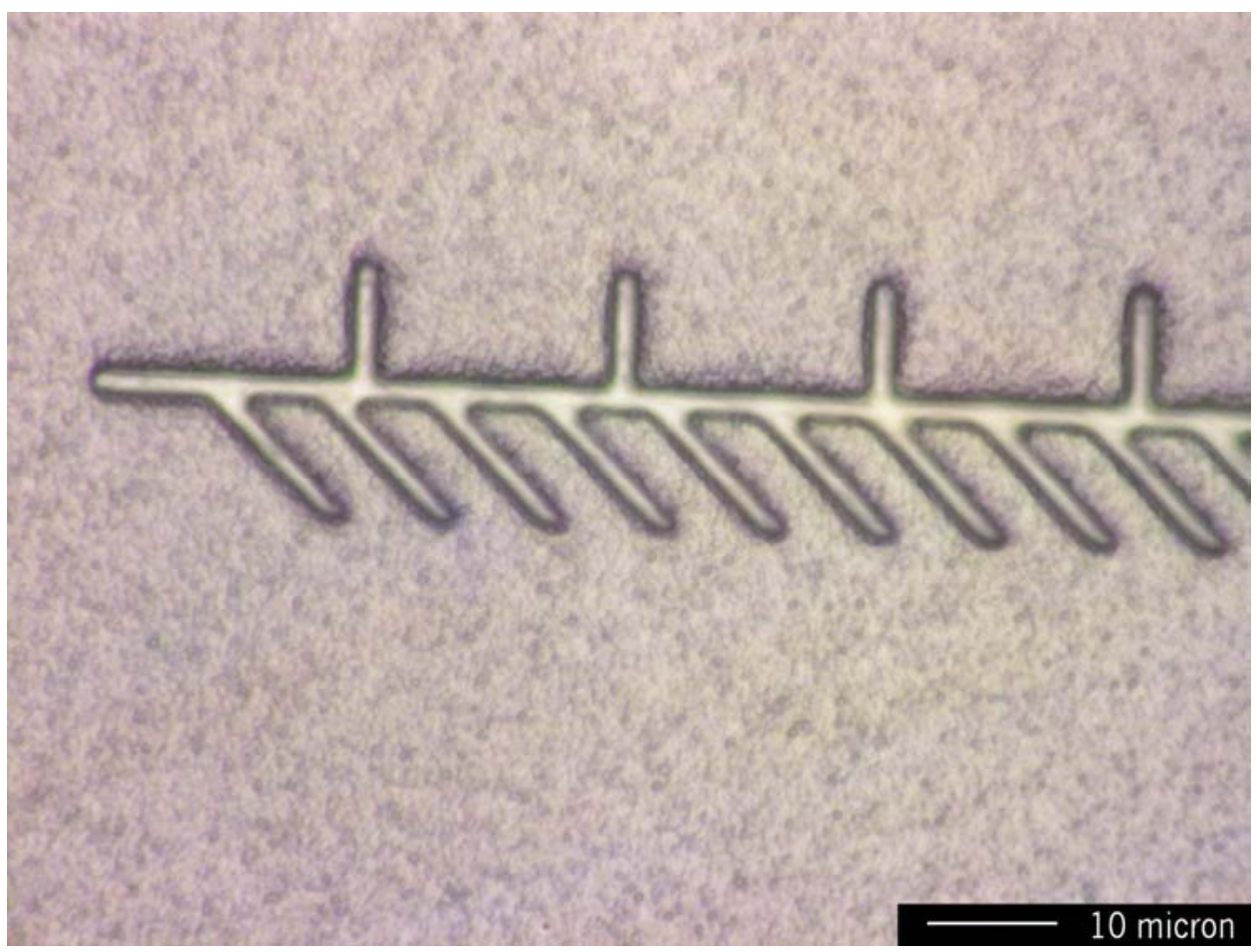

Figure A.7 Clear trench areas (polysilicon film, trial recipe-3 and photomask 'odd layer').

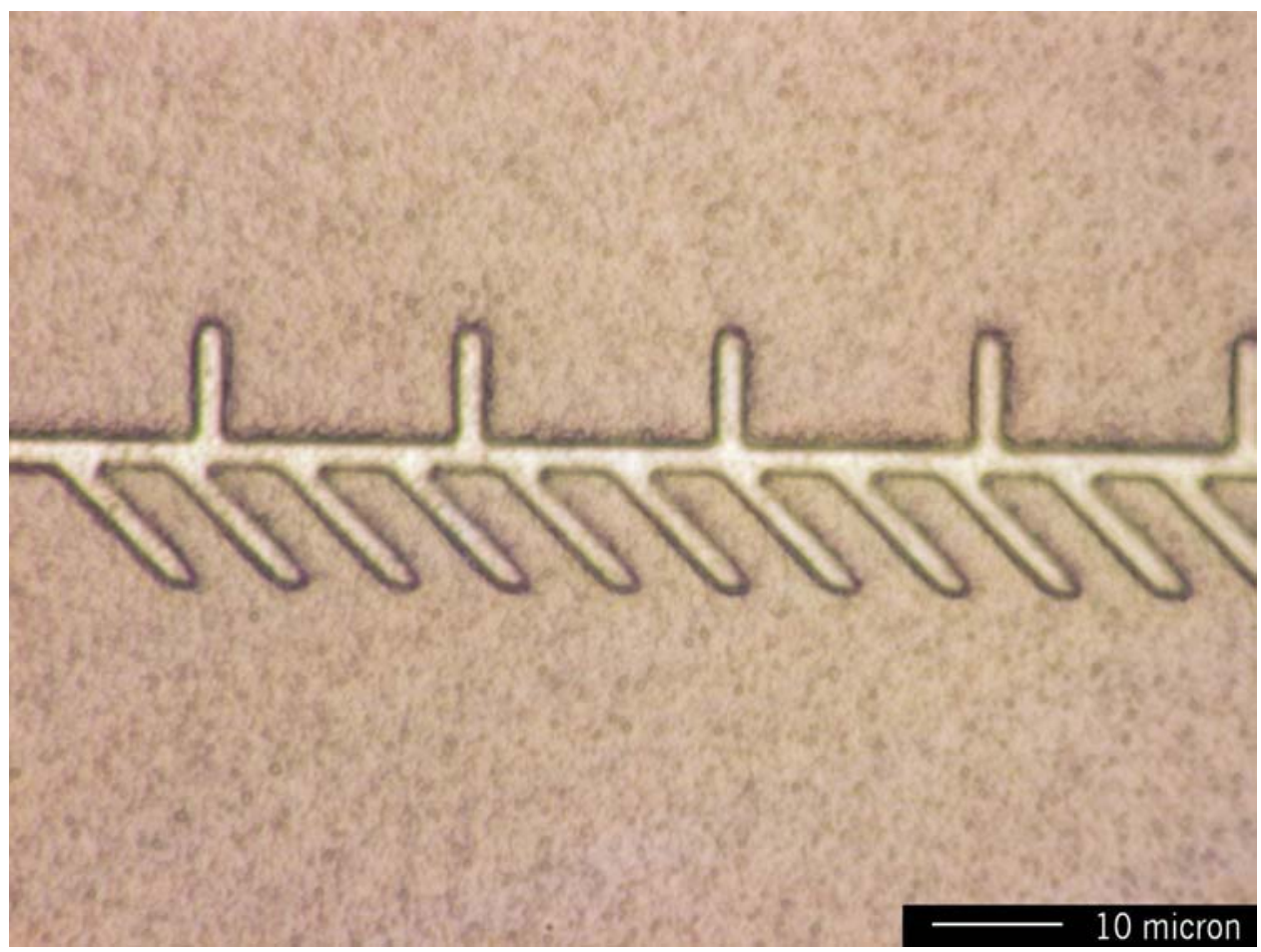

Figure A.8 Clear trench areas (sputtered silicon film, trial recipe-3 and photomask 'odd layer'). 


\begin{tabular}{|l|l|l|l|l|l|}
\hline & M1 $(\AA)$ & M2 $(\AA)$ & M3 $(\AA)$ & M4 $(\AA)$ & M5 $(\AA)$ \\
\hline After 20 seconds etching & 22357 & 23837 & 23223 & 22986 & 24039 \\
\hline After 40 seconds etching & 32588 & 34022 & 35217 & 33225 & 34921 \\
\hline $\begin{array}{l}\text { After removing photoresist } \\
\text { mask }\end{array}$ & 24157 & 25449 & 27051 & 24773 & 26378 \\
\hline
\end{tabular}

Table A.10 Etching results of the polysilicon film (trial recipe-3 and photomask 'odd layer').

\begin{tabular}{|c|c|c|c|c|c|}
\hline & $\begin{array}{l}\text { M1 } \\
(\AA / \mathrm{min})\end{array}$ & $\begin{array}{l}\text { M2 } \\
(\AA / \min )\end{array}$ & $\begin{array}{l}\text { M3 } \\
(\AA / \min )\end{array}$ & $\begin{array}{l}\text { M4 } \\
(\AA / \min )\end{array}$ & $\begin{array}{l}\text { M5 } \\
(\AA / \mathrm{min})\end{array}$ \\
\hline Photoresist Etch rate & 5354 & 5140 & 5751 & 5322 & 5186 \\
\hline $\begin{array}{l}\text { Silicon Etch rate in the first } 20 \\
\text { seconds }\end{array}$ & 36425 & 40652 & 39420 & 38280 & 41303 \\
\hline $\begin{array}{l}\text { Silicon Etch rate in the second } 20 \\
\text { seconds }\end{array}$ & 36046 & 35696 & 41733 & 36039 & 37831 \\
\hline
\end{tabular}

Table A.11 Etch rates of the polysilicon film (trial recipe-3 and photomask 'odd layer').

\begin{tabular}{|l|l|l|l|l|l|}
\hline & M1 $(\AA)$ & M2 $(\AA)$ & M3 $(\AA)$ & M4 $(\AA)$ & M5 $(\AA)$ \\
\hline After 20 seconds etching & 21633 & 22725 & 20699 & 21537 & 21504 \\
\hline After removing photoresist mask & 11459 & 12655 & 10708 & 11263 & 11476 \\
\hline
\end{tabular}

Table A.12 Etching results of the sputtered silicon film (trial recipe-3 and photomask 'odd layer').

\begin{tabular}{|c|c|c|c|c|c|}
\hline & $\begin{array}{l}\text { M1 } \\
(\AA / \min )\end{array}$ & $\begin{array}{l}\text { M2 } \\
(\AA / \min )\end{array}$ & $\begin{array}{l}\text { M3 } \\
(\AA / \min )\end{array}$ & $\begin{array}{l}\text { M4 } \\
(\AA / m i n)\end{array}$ & $\begin{array}{l}\text { M5 } \\
(\AA / \min )\end{array}$ \\
\hline Phot & 5478 & 5789 & 6028 & 5177 & 5916 \\
\hline $\begin{array}{l}\text { Silicon Etch rate in the first } 20 \\
\text { seconds }\end{array}$ & 34377 & 37965 & 32123 & 33788 & 34429 \\
\hline
\end{tabular}

Table A.13 Etch rates of the sputtered silicon film (trial recipe-3 and photomask 'odd layer'). 
Next, the trial recipe-3 was used to etch the sputtered silicon film that patterned by the photomask 'final structure'. The photoresist film was still $1.2 \mu \mathrm{m}$ thick. The top view of the anchor area before etching is presented in Figure A.9.

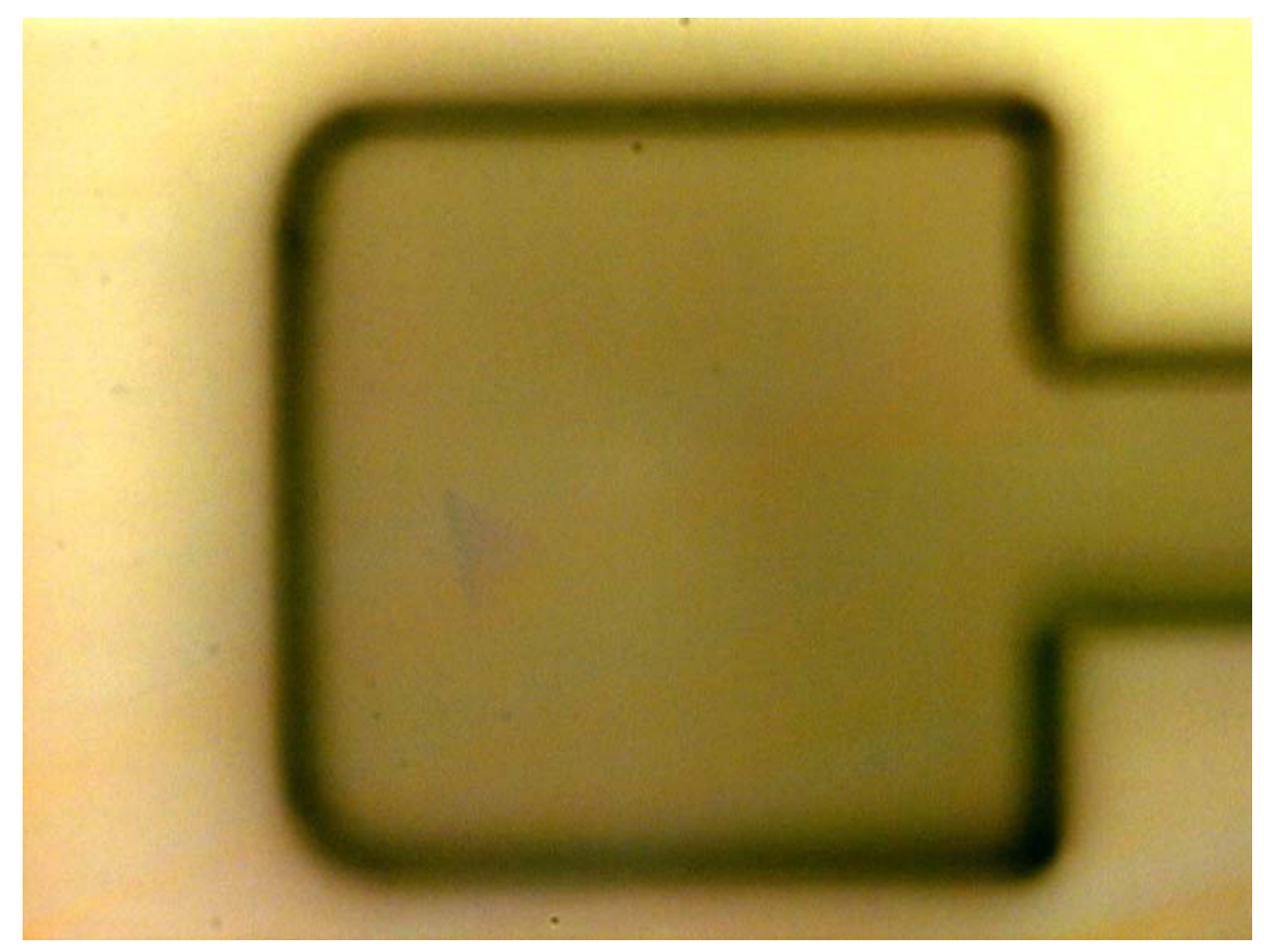

Figure A.9 Top view of the anchor area before etching the sputtered silicon film.

Two microns thick sputtered silicon film was etched for 20 seconds using trial recipe-3. After etching, no black silicon appeared in the etched area as shown in Figure A.10. In addition, no obvious fringes appeared across the wafer radius, which meant that the etching process was uniform. Step heights across the beams were measured for determining the etch rate.

The measured data and the calculated etch rates are listed in Table A.14 and Table A.15. In this case, the average etch rate for the sputtered silicon film and the photoresist film are $34468 \AA / \mathrm{min}$ and $6280 \AA / \mathrm{min}$, respectively. 


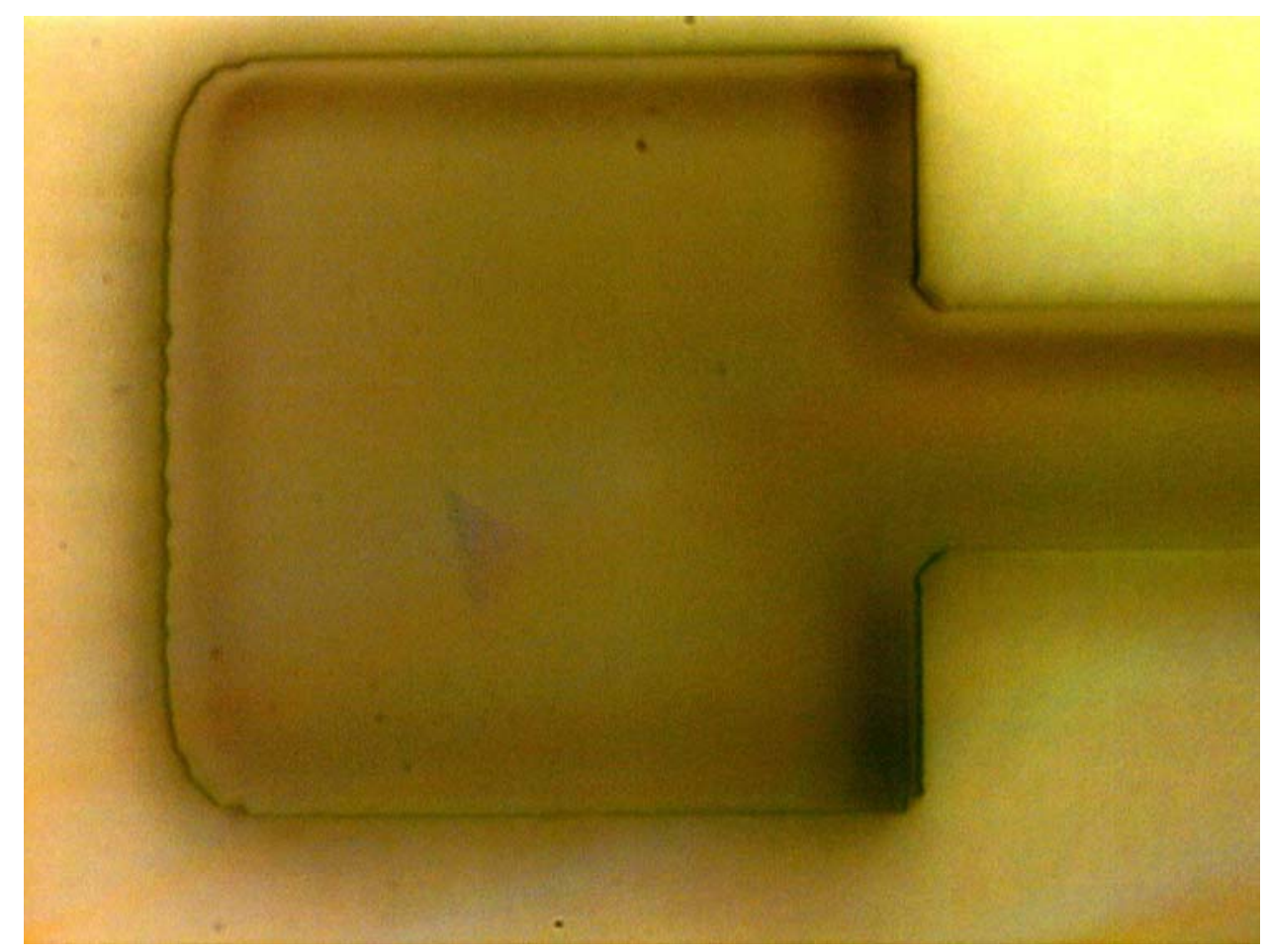

Figure A.10 Top view of the anchor area after etching the sputtered silicon film using trial recipe-3.

\begin{tabular}{|l|l|l|l|l|l|}
\hline & M1 $(\AA)$ & M2 $(\AA)$ & M3 $(\AA)$ & M4 $(\AA)$ & M5 $(\AA)$ \\
\hline After 20 seconds etching & 20704 & 21786 & 22306 & 20395 & 21790 \\
\hline After removing photoresist mask & 10754 & 11978 & 12315 & 10339 & 12061 \\
\hline
\end{tabular}

Table A.14 Etching results of the sputtered silicon film (trial recipe-3 and photomask 'final structure').

\begin{tabular}{|l|l|l|l|l|l|}
\hline & $\begin{array}{l}\text { M1 } \\
(\AA / \mathrm{min})\end{array}$ & $\begin{array}{l}\text { M2 } \\
(\AA / \mathrm{min})\end{array}$ & $\begin{array}{l}\text { M3 } \\
(\AA / \mathrm{min})\end{array}$ & $\begin{array}{l}\text { M4 } \\
(\AA / \mathrm{min})\end{array}$ & $\begin{array}{l}\text { M5 } \\
(\AA / \mathrm{min})\end{array}$ \\
\hline Photoresist Etch rate & 6149 & 6577 & 6028 & 5833 & 6812 \\
\hline $\begin{array}{l}\text { Silicon Etch rate in the first 20 } \\
\text { seconds }\end{array}$ & 32262 & 35934 & 36945 & 31017 & 36183 \\
\hline
\end{tabular}

Table A.15 Etch rates of the sputtered silicon film (trial recipe-3 and photomask 'final structure'). 
So far, the trial recipe-3 is a successful one for etching the sputtered silicon film and the polysilicon film. The last step is to etch the silicon dioxide film with trial recipe-3 to determine its etch rate, and then to evaluate the selectivity of the trial recipe-3.

Silicon dioxide films were thermally grown on the testing wafer with a target thickness of $2 \mu \mathrm{m}$. The oxide film was etched for 30 seconds in the first run. After measuring the oxide film thickness, it was etched for another 30 seconds.

Table A.16 lists the film thickness measured after each etching step and the corresponding etch rates of the oxide film are presented in Table A.17.

\begin{tabular}{|l|l|l|l|l|l|}
\hline & M1 $(\AA)$ & M2 $(\AA)$ & M3 $(\AA)$ & M4 $(\AA)$ & M5 $(\AA)$ \\
\hline Original thickness & 19937 & 19934 & 19928 & 19946 & 19926 \\
\hline After 30 seconds etching & 18658 & 18564 & 18415 & 18519 & 18422 \\
\hline After 60 seconds etching & 17381 & 17245 & 17001 & 17221 & 17055 \\
\hline
\end{tabular}

Table A.16 Etching results of the oxide film using trial recipe-3.

\begin{tabular}{|l|c|c|c|c|c|}
\hline & $\begin{array}{c}\text { M1 } \\
(\AA / \mathrm{min})\end{array}$ & $\begin{array}{c}\mathrm{M} 2 \\
(\AA / \mathrm{min})\end{array}$ & $\begin{array}{c}\mathrm{M} 3 \\
(\AA / \mathrm{min})\end{array}$ & $\begin{array}{c}\mathrm{M} 4 \\
(\AA / \mathrm{min})\end{array}$ & $\begin{array}{c}\text { M5 } \\
(\AA / \mathrm{min})\end{array}$ \\
\hline $\begin{array}{l}\text { Oxide Etch rate in the first 30 } \\
\text { seconds }\end{array}$ & 2558 & 2741 & 3026 & 2854 & 3008 \\
\hline $\begin{array}{l}\text { Oxide Etch rate in the second } \\
30 \text { seconds }\end{array}$ & 2554 & 2638 & 2829 & 2596 & 2735 \\
\hline
\end{tabular}

Table A.17 Etch rate of the oxide film using trial recipe-3.

The data shown in Table A.17 indicates that the etch rate of the oxide film is consistent within different etching steps. The average etch rates is $2670 \AA / \mathrm{min}$ with a uniformity of $5.15 \%$. Table A.18 presents the summary of the etching properties of the trial recipe-3. 


\begin{tabular}{|l|c|c|}
\hline & Average etch rate $(\AA / \mathrm{min})$ & $\begin{array}{l}\text { Etching uniformity } \\
(\%)\end{array}$ \\
\hline $\begin{array}{l}\text { Polysilicon with ‘odd layer' etching } \\
\text { mask }\end{array}$ & 37469 & 8.06 \\
\hline $\begin{array}{l}\text { Polysilicon with 'final structure' } \\
\text { etching mask }\end{array}$ & $\mathrm{X}$ & $\mathrm{X}$ \\
\hline $\begin{array}{l}\text { Sputtered silicon with ‘odd layer' } \\
\text { etching mask }\end{array}$ & 34536 & 7.46 \\
\hline $\begin{array}{l}\text { Sputtered silicon with ‘final } \\
\text { structure' etching mask }\end{array}$ & 34468 & 7.50 \\
\hline $\begin{array}{l}\text { Photoresist as ‘odd layer' etching } \\
\text { mask }\end{array}$ & 5677 & 7.79 \\
\hline $\begin{array}{l}\text { Photoresist as 'final structure' } \\
\text { etching mask }\end{array}$ & 6280 & 5.15 \\
\hline Thermal silicon dioxide & 2670 & \\
\hline
\end{tabular}

Table A.18 Summary of the etch rates for different materials using the trial recipe-3.

In the sense of good etching process, the etching uniformity should be smaller than $3 \%$. The etching uniformity of the trial recipe-3 is relatively larger than this requirement. However, it is still acceptable because the steep etching gradient only appears around the wafer edge and the etching profile in the central area is relatively consistent. This guarantees that ideal structures can be obtained at least around the wafer center. Since the etch rate is slow in the wafer center, it will be used to control the etching time for the latter silicon etching processes.

\section{A.3 Photoresist application}

The application of the photoresist onto the silicon films will directly determine the mechanical properties of the microstructures. This process is done with physical methods, namely taking advantage of the flowing nature of the liquid photoresist to fill in trenches. Inevitably, there will be defects existent in the trenches or in the interface between silicon 
and photoresist. Thus, the effect of spinning speed and other parameters on the photoresist application will be studied to ensure successful filling.

\section{A.3.1 Photoresist film thickness versus spin speed}

First, the relation between the photoresist thickness and the spinning speed was studied using Shipley 1813 photoresist and AZ9260 photoresist. AZ9260 photoresist is thicker than Shipley 1813 and can be used for forming thicker photoresist films. The photoresist thickness is related to the photoresist amount applied on the wafer and the spinning speed.

The applied photoresist amount was fixed by setting the pouring time to be 0.3 $\mathrm{sec}$, and then changed the spinning speed from $1000 \mathrm{rpm}$ to $7500 \mathrm{rpm}$ with a constant spinning time of 30 seconds. Table A.19 lists the film thickness measured using Ellipsometer for Shipley 1813 photoresist.

\begin{tabular}{|l|c|c|c|c|c|}
\hline & $1000 \mathrm{rpm}$ & $2500 \mathrm{rpm}$ & $4000 \mathrm{rpm}$ & $7000 \mathrm{rpm}$ & $7500 \mathrm{rpm}$ \\
\hline M1 $(\AA)$ & 45037 & 16238 & 12343 & 10223 & 9637 \\
\hline M2 $(\AA)$ & 47685 & 17589 & 11817 & 10956 & 9544 \\
\hline M3 $(\AA)$ & 49332 & 17223 & 12064 & 10737 & 9125 \\
\hline M4 $(\AA)$ & 48098 & 18034 & 11728 & 10173 & 10201 \\
\hline M5 $(\AA)$ & 49523 & 17888 & 12404 & 10759 & 9755 \\
\hline Mean $(\AA)$ & 47935 & 17394 & 12071 & 10570 & 9652 \\
\hline Uniformity & $4.68 \%$ & $5.16 \%$ & $2.55 \%$ & $3.70 \%$ & $5.57 \%$ \\
\hline
\end{tabular}

Table A.19 Photoresist thicknesses vs. spinning speed.

The AZ 9260 photoresist was applied manually using a pouring gun, and the photoresist amount was control by the shutter switch. The spinning time was 60 seconds for all of the photoresist application processes. Figure A.11 and Figure A.12 show the variance of the average thickness of the photoresist films versus the spinning speed. 


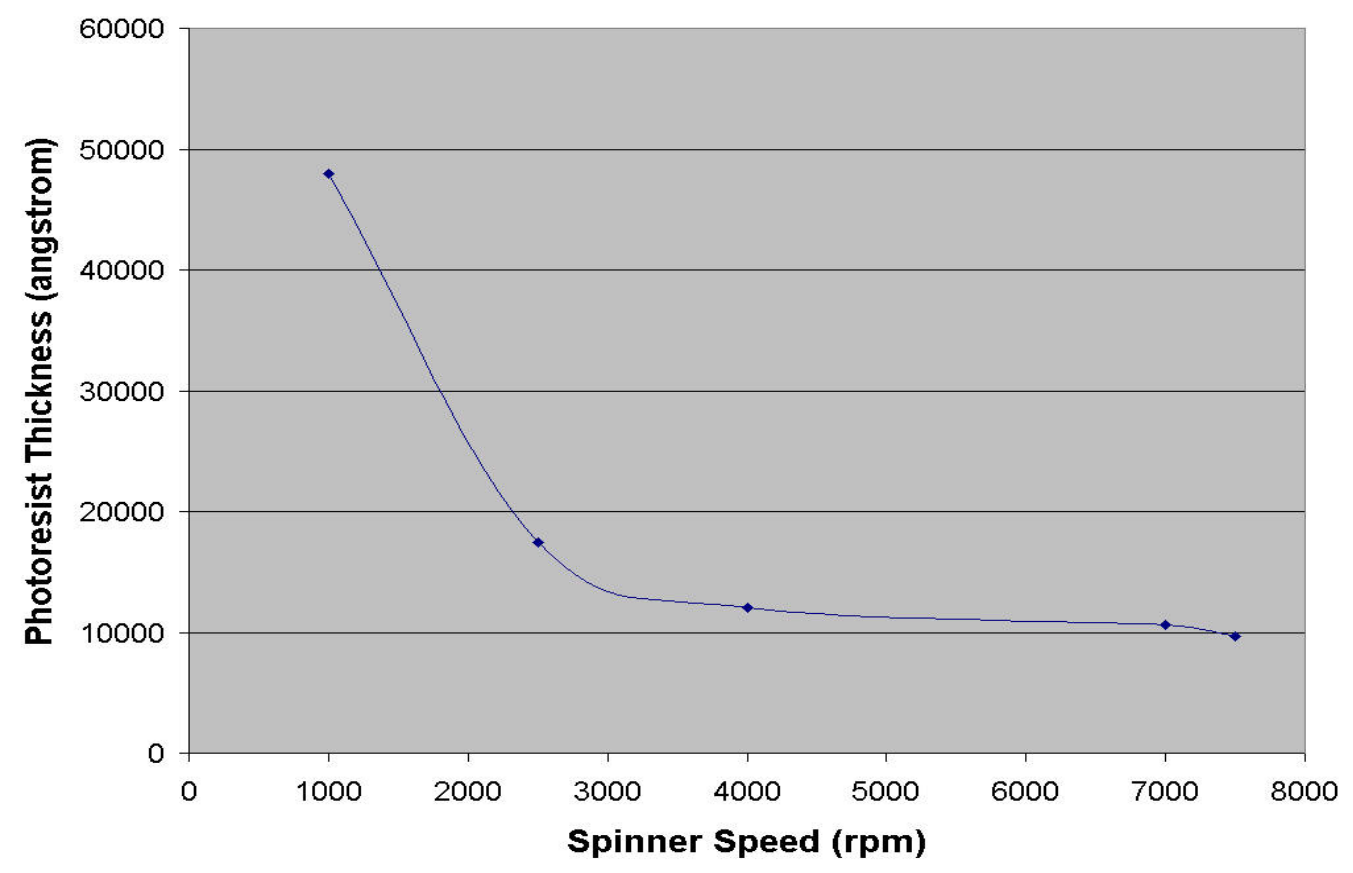

Figure A.11 Photoresist thickness vs. spinning speed, Shipley 1813

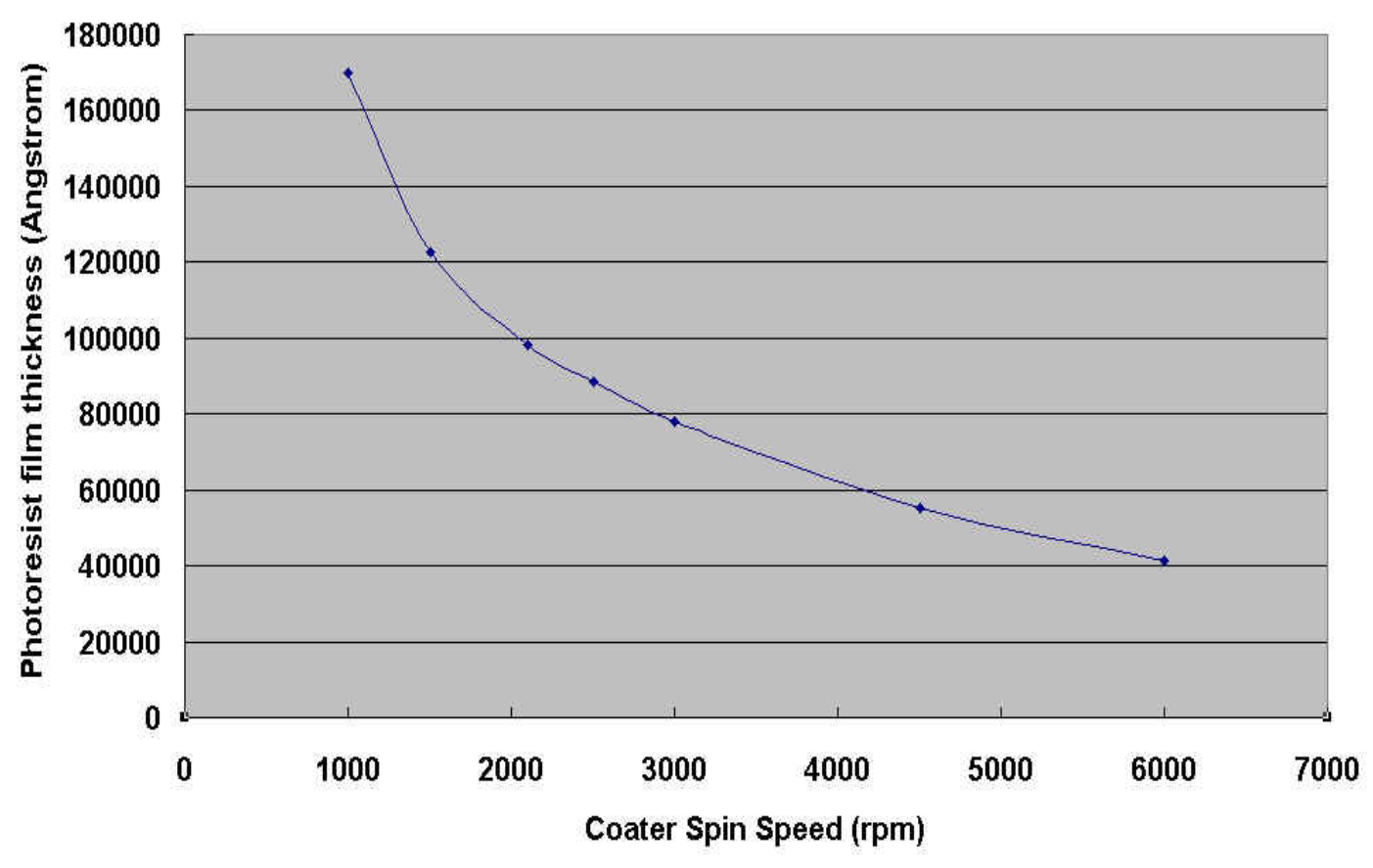

Figure A.12 Photoresist thickness vs. spinning speed, AZ 9260 
The properties of the photoresist films are partially correlated to the photoresist state in its container. Sometimes, the photoresist is thicker at the bottom of the container. This will make the photoresist film non-uniform. Therefore, the data in Figure A.11 and Figure A.12 are only for reference and the actual thickness of the photoresist film has to be measured.

\section{A.3.2 Filling photoresist in trenches}

This photoresist filling process was conducted using a spin coater manufactured by Solitec. Table A.20 shows the parameters that can be adjusted for controlling the film thickness. The data in Table A.20 are used for general photoresist application, which will produce $1.2 \mu \mathrm{m}$ thick photoresist film.

\begin{tabular}{|c|c|c|c|c|}
\hline & $\begin{array}{c}\text { Pre- photoresist } \\
\text { application }\end{array}$ & Pouring photoresist & Pre-spin & Spin \\
\hline Spin speed (rpm) & 2000 & 0 & 0 & 4000 \\
\hline Time (second) & 30 & 0.3 & 0 & 30 \\
\hline
\end{tabular}

Table A.20 Spin coater parameters for depositing $1.2 \mu \mathrm{m}$ thick photoresist film, Shipley 1813.

The pre-photoresist application step was used for adjusting the wafer position to make sure it was located in the center of the chunk stage. In the pouring photoresist step, step time determined the photoresist amount applied on the wafer. The pre-spin step was associated with the previous step to ensure photoresist flow all over the wafer and uniformly cover the whole wafer surface. The spinning speed in this step was set to be much lower than that in the final step. Lower spinning speed helped the applied photoresist to flow and gave it enough time to sink in the trenches. After this step, final spinning was run with different speeds to form photoresist film with ideal thickness. 
The filling profile was investigated with a relatively larger feature size. The testing wafer was prepared with $2 \mu \mathrm{m}$ thick thermal oxide film and $2.5 \mu \mathrm{m}$ thick polysilicon film deposited using LPCVD. The polysilicon film was etched using a chlorine-based recipe and a photomask that could form $6 \mu \mathrm{m}$ wide spacing. As shown in Table A.21, the testing process applied more photoresist on the wafer by setting the pouring time to be $0.4 \mathrm{sec}$ and pre-spin the wafer for $10 \mathrm{sec}$ with a speed of $500 \mathrm{rpm}$. This was immediately followed by the final spin for $30 \mathrm{sec}$ with a speed of $1500 \mathrm{rpm}$. Then, the wafer was loaded in an oven for 60 mins soft bake. Nanpspec4000 measurements showed that the thickness of the photoresist film was $41357 \pm 1244 \AA$. The final profile was observed using SEM.

Figure A.13 shows the SEM picture. The distance between the two columns is $7 \mu \mathrm{m}$. The photoresist fills in the space between two polysilicon columns very well. No visible gaps or defects exist in the interface area.

\begin{tabular}{|c|c|c|c|c|}
\hline & $\begin{array}{c}\text { Pre- photoresist } \\
\text { application }\end{array}$ & Pouring photoresist & Pre-spin & Spin \\
\hline Spinning speed (rpm) & 2000 & 0 & 500 & 1500 \\
\hline Time (second) & 30 & 0.5 & 10 & 30 \\
\hline
\end{tabular}

Table A.21 Spin coater parameters for depositing $4.1 \mu \mathrm{m}$ thick Shipley 1813 photoresist. 


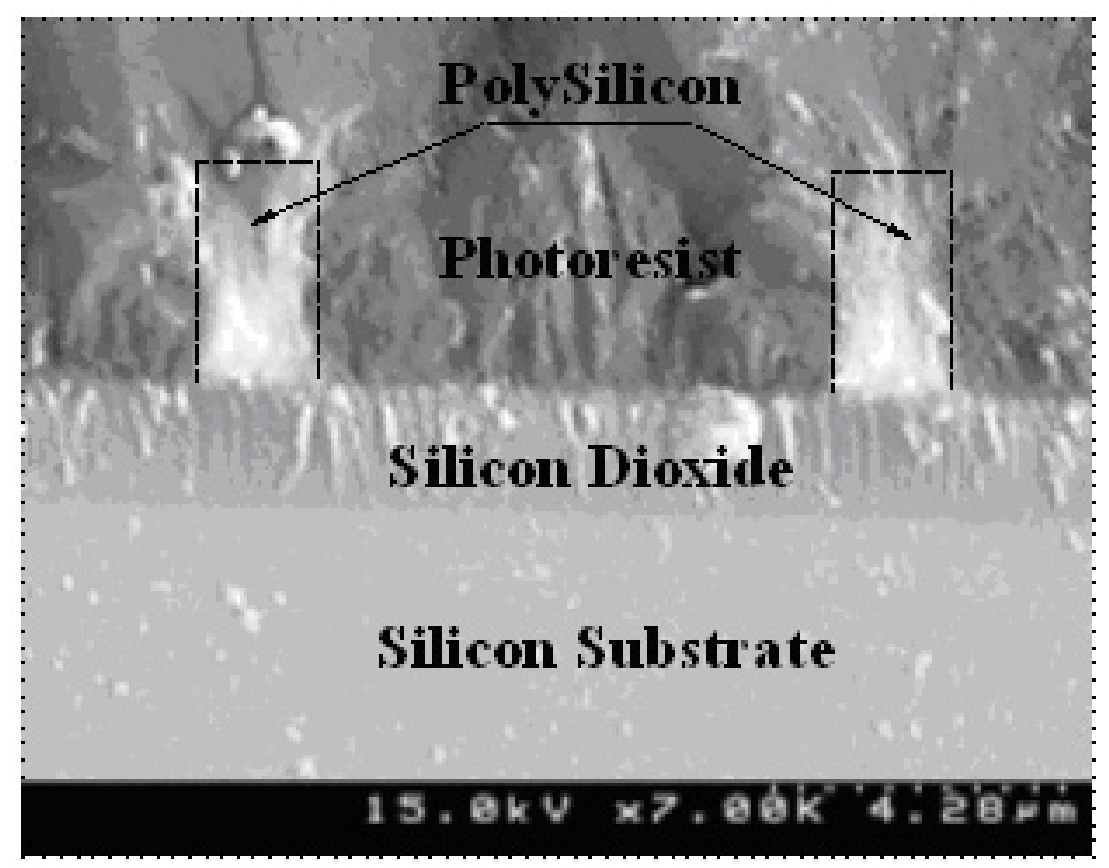

Figure A.13 Feature profile after filling photoresist in the $7 \mu \mathrm{m}$ wide gap.

Next, the same test was run using photomask 'odd layer'. Referred to the data shown in Figure A.11, the final spinning speed and the pouring time were set to be 7000rpm and $0.3 \mathrm{sec}$ for a target film thickness of $0.9 \mu \mathrm{m}$. Table A.22 lists the parameters used for this test.

\begin{tabular}{|c|c|c|c|c|}
\hline & $\begin{array}{c}\text { Pre- photoresist } \\
\text { application }\end{array}$ & Pouring photoresist & Pre-spin & Spin \\
\hline Spin speed (rpm) & 2000 & 0 & 500 & 7000 \\
\hline Time (second) & 30 & 0.3 & 10 & 30 \\
\hline
\end{tabular}

Table A.22 Spin coater parameters for depositing $0.9 \mu \mathrm{m}$ thick Shipley 1813 photoresist.

After applying photoresist, photomask 'interface' was used to retain the photoresist films on the top of the microstructure areas. Figure A.14 presents a typical top view of the feature area. 


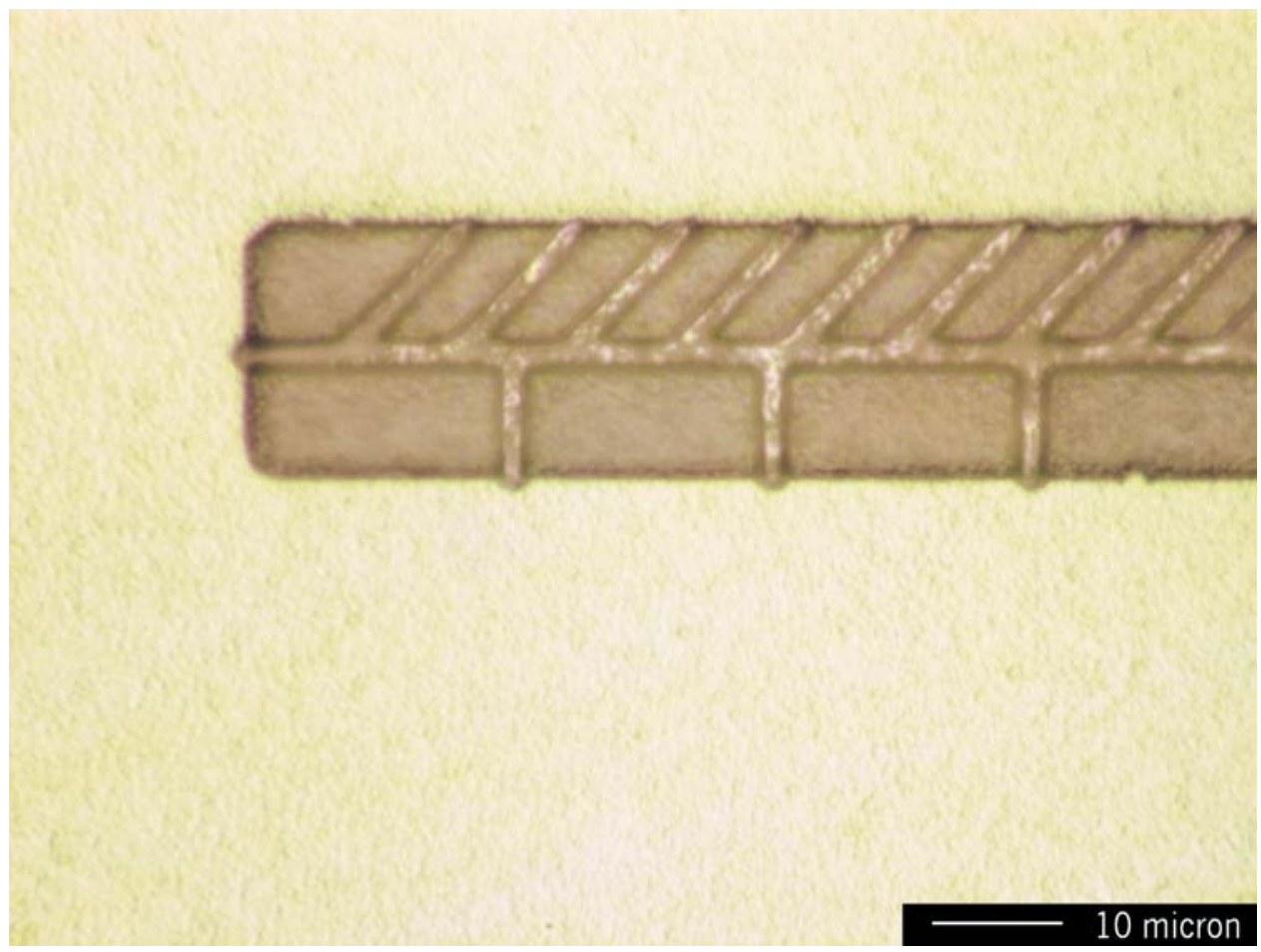

Figure A.14 Photoresist filling result after applying photoresist using the parameters shown in Table A.22.

The photoresist did not fill in the trenches perfectly. It was not continuously distributed in most of the trenches. The shorter pre-spin time might be one reason for causing this problem because short pre-spin time makes it hard for the photoresist to sink and flow inside the trenches, and then cannot completely fill the trenches. Thicker film can help to improve the filling. Increasing the amount of photoresist or reducing the final spinning speed can make the film thicker, and the final spinning speed is relatively more critical.

After several adjustments, the parameters in Table A.23 produced best filling result as shown in Figure A.15. In this case, the thickness of the photoresist film was about $10500 \AA$. 
Even though some gaps still can be found around the trench corners, they are not significant compared to the large area of the continuous photoresist in the trenches and the filling result is acceptable.

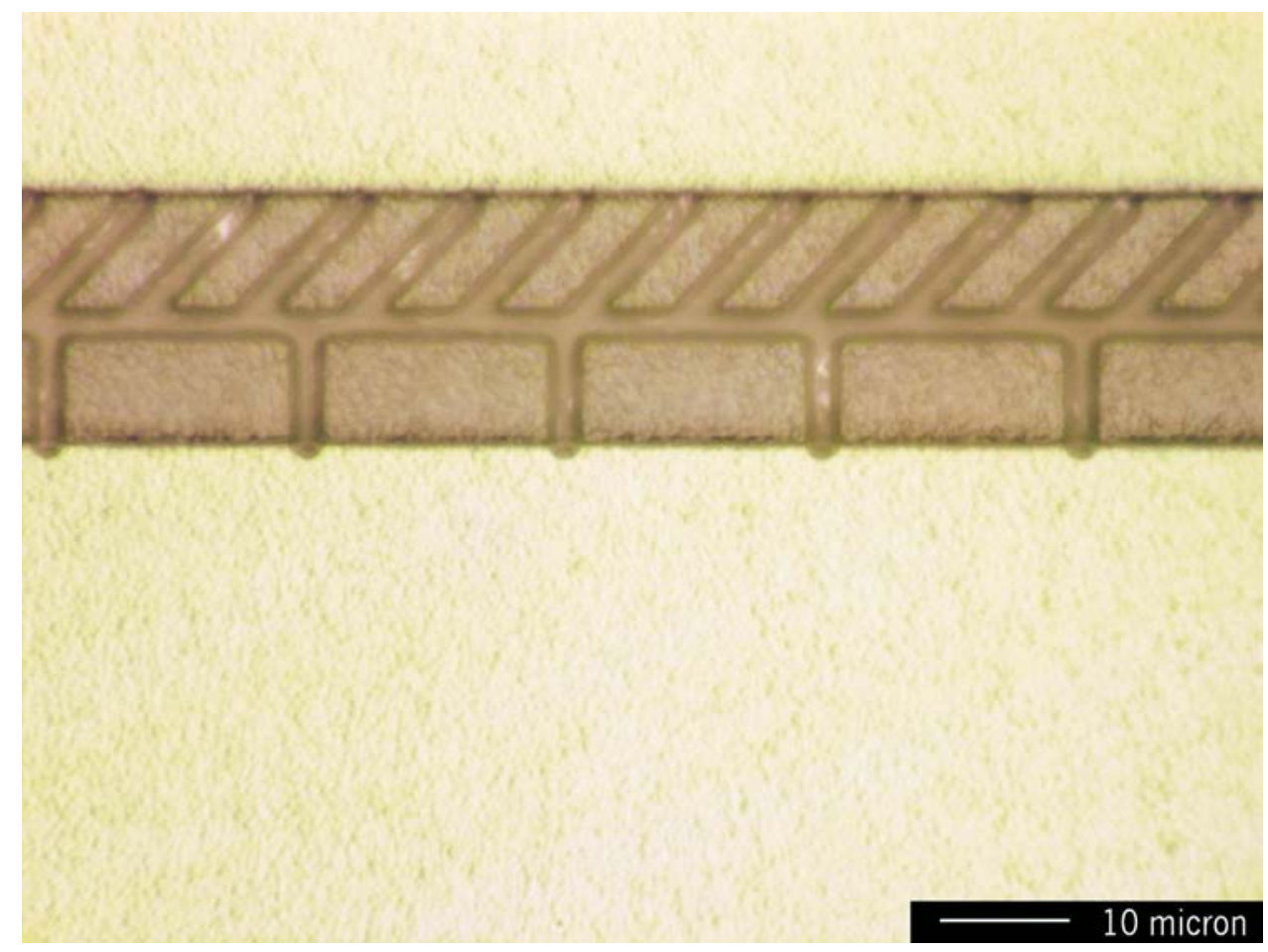

Figure A.15 Photoresist filling result using the parameters shown in Table 3.30.

\begin{tabular}{|c|c|c|c|c|}
\hline & $\begin{array}{c}\text { Pre- photoresist } \\
\text { application }\end{array}$ & Pouring photoresist & Pre-spin & Spin \\
\hline Spin speed (rpm) & 2000 & 0 & 1000 & 5500 \\
\hline Time (second) & 30 & 0.4 & 15 & 30 \\
\hline
\end{tabular}

Table A.23 Spin coater parameters for depositing $1.05 \mu \mathrm{m}$ thick Shipley 1813 photoresist. 


\section{A.3.3 Etch interface photoresist}

Once the process reaches the step shown in Figure 2.4(6), the photoresist film has to be etched down to the silicon surface. This process requires that the etching recipe have higher selectivity between photoresist and silicon.

This recipe was developed using RIE tool Tegal803. The recipe was run under pressure of 3 Torr and power of 100Watts. The temperature in the etching chamber was $16{ }^{0} \mathrm{C}$. The etching plasmas were comprised of $\mathrm{CHF}_{3}, \mathrm{O}_{2}$ and $\mathrm{He}$ with the flow rate of 10 , 40 and $70 \mathrm{sccm}$, respectively. The etching test was done with a $1.7 \mu \mathrm{m}$ thick photoresist film deposited on the top of a $2.5 \mu \mathrm{m}$ thick polysilicon film. The photoresist film and the polysilicon film were not covered by any masks. The photoresist film was etched for 2 minutes. Table A.24 shows the film thicknesses measured before and after etching. The average etch rates for photoresist and polysilicon are $4781 \AA / \mathrm{min}$ and $469 \AA / \mathrm{min}$. The selectivity ratio is almost equal to 10 and it is good enough to protect silicon during the photoresist etching.

\begin{tabular}{|c|l|c|c|c|c|c|}
\hline & & $\mathrm{M} 1$ & $\mathrm{M} 2$ & $\mathrm{M} 3(\AA)$ & $\mathrm{M} 4(\AA)$ & $\mathrm{M} 5(\AA)$ \\
\hline Original thickness $(\AA)$ & Photoresist & 17339 & 17223 & 17109 & 17283 & 17158 \\
\cline { 2 - 7 } & Polysilicon & 25065 & 24999 & 25083 & 25102 & 24989 \\
\hline $\begin{array}{c}\text { After 2-minute etching } \\
(\AA)\end{array}$ & Photoresist & 6951 & 8019 & 8041 & 7393 & 7894 \\
\cline { 2 - 7 } & Polysilicon & 24202 & 24011 & 24197 & 24106 & 24035 \\
\hline Etch rate $(\AA / \mathrm{min})$ & Photoresist & 5194 & 4602 & 4534 & 4945 & 4632 \\
\cline { 2 - 7 } & Polysilicon & 432 & 494 & 443 & 498 & 477 \\
\hline
\end{tabular}

Table A.24 Etching results and etch rates for polysilicon and photoresist using $\mathrm{CHF} 3 / \mathrm{O}_{2} / \mathrm{He}$ recipe. 


\section{Bibliography}

1. J.D. Currey and J.D. Taylor, "The Mechanical Behavior of Some Molluscan Hard Tissues”, Journal of Zoology, London, Vol.173, pp.395-, 1974.

2. J.D. Currey, "A Comparison of the Strength of Echinoderm Spines and Mollusc Shells", Journal of the Marine Biological Association of the United Kingdom, Vol.55, pp.419-, 1975.

3. J.D. Currey and A.K. Kohn, "Fracture in the Crossed-lamellar structure of Conus Shells", Journal of Materials Science, 11, pp.1615-, 1976.

4. J.D. Currey, "The Mechanical Properties of biological materials", CUP, pp.75-97, 1980.

5. S. Kamat, "Toughening Mechanisms in Laminated Composites: A Biomimetic Study in Mollusk shells”, Ph.D. Dissertation, Case Western Reserve University, August 2000.

6. S. Kamat, X. Su, R. Ballarini and A.H. Heuer, "Structural Basis for the Fracture Toughness of the shell of the Conch Strombus Gigas”, Nature, Vol.405, pp.10361040, June 2000.

7. L.T. Kuhn-Spearing, H. Kessler, E. Chateau, R. Ballarini and A.H. Heuer, "Fracture Mechanisms of the Strombus Gigas Conch Shell: Implications for the Design of Brittle Laminates”, Journal of Materials Science, Vol.31, pp.6583-6594, 1996.

8. H. Kessler, R. Ballarini, R.L. Mullen, L.T. Kuhn and A.H. Heuer, "A Biomimetic Example of Brittle Toughening: (I) Steady State Multiple Cracking”, Computational Materials Science, Vol.5, pp.157-166, 1996. 
9. S. Kamat, H. Kessler, R. Ballarini, M. Nassirou and A.H. Heuer, "Fracture Mechanisms of the Strombus Gigas conch Shell: (II) Micromechanics Analyses of Multiple Cracking and Large-scale Crack Bridging”, Acta Materialia, Vol.52, pp.2395-2406, 2004.

10. P. Calvert, J. Cesarano, H. Chandra, H Denham, S. Kasichainula and R. Vaidyanathan, "Toughness in Synthetic and Biological Multilayered Systems", Philosophical Transactions: Mathematical, Physical \& Engineering Sciences, The Royal Society of London, Vol.360, pp.199-209, 2002.

11. W.J. Clegg, K. Kendall, N.McN. Alford, T.W. Button and J.D. Birchall, “A Simple Way to Make Tough Ceramics”, Nature, Vol.347, pp.455-457, 1990.

12. H. Lorenz, M. Laudon, and P. Renaud, "Mechanical characterization of a new highaspect-ratio near UV-photoresist”, Microelectronic Engineering, Vol.41/42, pp.371$374,1998$.

13. P. Hudek, P. Hrkut, M. Drzik, I. Kostic, M. Belov, J. Torres, A. Degen, J. Butschke, A. Ehrmann and E. Haugeneder, "Directly Sputtered Stress-compensated Carbon Protective Layer for Silicon Stencil Masks", The Journal of Vacuum Science and Technology B, Vol.17 (6), pp.3127-3131, 1999.

14. C.A. Zorman, A.J. Fleischman, A.S. Dewa, M. Mehregany, C. Jacob, S. Nishino and P. Pirouz, "Epitaxial growth of 3C-SiC films on 4 in. diam (100) silicon wafers by atmospheric pressure chemical vapor deposition", Journal of Applied Physics, Vol. 78, pp.5136-5138, 1995. 
15. A.J. Fleischman, "Silicon Carbide Thin Films for High Temperature Microelectromechanical Systems", Ph.D. Dissertation, Case Western Reserve University, May 1999

16. C.A. Zorman, S. Rajgopal, X.A. Fu, R. Jezeski, J. Melzak and M. Mehregany, "Deposition of polycrystalline 3C-SiC films on $100 \mathrm{~mm}$ diameter (100) Si wafers in a large-volume LPCVD furnace", Electrochemical and Solid State Letters, Vol.5, pp. G99-G101, 2002.

17. Y.T. Yang, K. L. Ekinci, X.M.H. Huang, L.M. Schiavone, C.A. Zorman, M. Mehregany, and M.L. Roukes, "Monocrystalline Silicon Carbide NEMS", Applied Physics Letters, Vol.78, pp.162-164, 2001.

18. I.M. Daniel and O. Ishai, "Engineering Mechanics of Composite Materials", Oxford University Press, 1994.

19. M.W. Hyer, "Stress Analysis of Fiber-reinforced Composite Materials", WCB McGraw-Hill Press, 1998.

20. K.A. Honer, "Surface Micromachining Techniques for Integrated Microsystems", Ph.D. Dissertation, Stanford University, March 2001.

21. H. Jansen, M. de Boer, R. Legtenberg and M. Elwenspoek, "The Black Silicon Method: a Universal Method for Determining the Parameter Setting of a Fluorinebased Reactive Ion Etcher in Deep Silicon trench Etching with Profile control”, Journal of Micromechanics and Microengineering, Vol.5, pp.115-120, 1995.

22. H. Jansen, M. de Boer, J. Burger, R. Legtenberg and M. Elwenspoek, "The Black Silicon Method II: The Effect of Mask Material and Loading on the Reactive Ion 
Etching of Deep Silicon Trenches", Microelectronic Engineering, Vol.27, pp.475480, 1995.

23. H. Jansen, H. Gardeniers, M. de Boer, M. Elwenspoek and J. Fluitman, “A Survey on the Reactive Ion Etching of Silicon in Microtechnology", Journal of Micromechanics and Microengineering, Vol.6, pp.14-28, 1996.

24. I. Sneddon, "The Relation between Load and Penetration in the Axisymmetric Boussinesq Problem for a Punch of Arbitrary Profile", International Journal of Engineering Science, Vol.3, pp.47-57, 1965.

25. G. Pharr, W. Oliver and F. Brotzen, "On the Generality of the Relationship among Contact Stiffness, Contact Area, and Elastic Modulus during Indentation", Journal of Materials Research, Vol.7, No.3, pp.613-617, 1992.

26. W. Oliver and G. Pharr, "An Improved Technique for Determining Hardness and Elastic Modulus Using Load and Displacement Sensing Indentation Experiments", Journal of Materials Research, Vol.7, No.6, pp.1564-1583, 1992.

27. H. Kahn, R. Ballarini and A.H. Heuer, "Cyclic Loading of Polysilicon I: Effects of Mean Stress and Stress Amplitude," submitted to Acta Metallurgica et Materialia, 2005.

28. H. Kahn, R. Ballarini, J.J. Bellante and A.H. Heuer, "Fatigue Failure in Polysilicon not Due to Simple Stress Corrosion Cracking", Science, 298, 1215-1218, 2002.

29. M.J. Demkowicz and A.S. Argon, "High-Density Liquidlike Component Facilitates Plastic Flow in Model Amorphous Silicon System," Physical Review Letters 93, 2004, 25505.

30. F.H. Stillinger and T.A. Weber, Physical Review B, 37, 4132, 1988. 
31. ABAQUS, ABAQUS, Inc., Providence, RI.

32. R.L. Mullen, R. Ballarini and Y.Yin, "Monte-Carlo Simulation of Effective Elastic Constants of Polycrystalline Thin Films," Acta Metallurgica et Materialia, 45, 2247$2255,1997$. 


\section{VITA}

NAME: $\quad$ Li Chen

BIRTH: $\quad$ June 18,1973

Tianjin, CHINA

EDUCATION: $\quad$ Ph.D. --Engineering Mechanics, Department of Civil Engineering

Case Western Reserve University, Cleveland, OHIO. (05/24/2005)

M.S. --Engineering Mechanics, Department of Civil Engineering

Case Western Reserve University, Cleveland, OHIO. (05/31/2000)

B.S. --Engineering Mechanics, Department of Engineering Mechanics

Tsinghua University, Beijing, P. R. CHINA. (07/04/1997)

AWARDS:

Higley Scholarship, Case Western Reserve University, (9/01/2003)

Case Prime Fellowship, Case Western Reserve University, (6/01/2001)

Roy Harley Prize to a Promising Graduate Student, Case Western Reserve University, $(5 / 31 / 2000)$

PUBLICATIONS: $\quad$ 1. L. Chen, R. Ballarini, and M. Grigoriu, "Crack Propagation in a Material with Random Fracture Toughness,” International Journal of Fracture 125: 353-369, 2004.

2. L. Chen, R. Ballarini and A.H. Heuer, "A Bio-inspired MEMS Composite," in preparation.

3. L. Chen and R. Ballarini, "Cyclic Loading-Induced Strengthening of MEMS Polysilicon," in preparation. 